siollity.

LLL- -17491

DE34 010703

\title{
MECHANICAL TRANSPORT IN TWO-DIMENSIONAL NETWORKS OIS FRACTURES
}

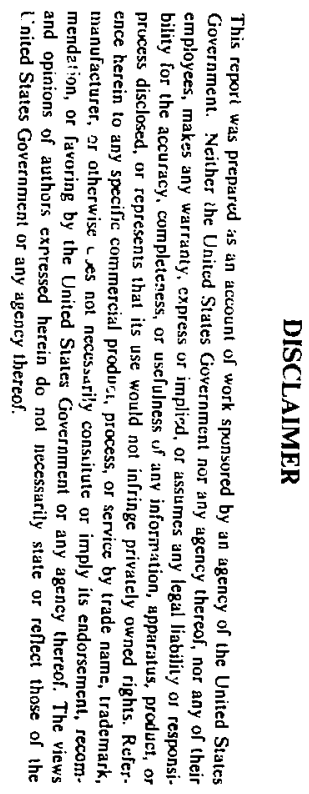

\author{
Howard K. Endo
}

Earth Science Division

Lawrence Berkeley Laboraiory

University of California

Berkeley, California 94720

Ph.D. Thesis

April 1984

This work was supported by the U. S. Department of Energy ur'ter Contract Number DEACO3-76SF00098. 


\title{
MECHANICAL TRANSPORT IN TWO-DIMENSIONAL NETWORKS OF FRACTURES
}

\author{
Howard K. Endo
}

Ph.D.

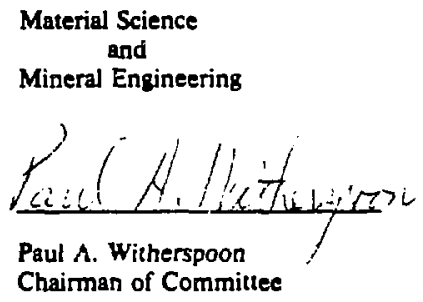

\begin{abstract}
The objectives of this research are to evaluate directional mechanical transport parameters for anisotropic fracture systems, and to determine if fracture systems behave like equivaient porous media. The tracer experiments used to measure directional tortuosity, longitudinal geometric dispersivity, and hydraulic effective porosity are conducted with a uniform flow field and measurements are made from the fluid flowing within a test section where linear length of Iravel is constant. Since fluid flow and mechanical transport are coupled processes, the directional variations of specific discharge and hydraulic effective porosity are measured in regions with constant hydraulic gradients to evaluate porous medium equivalence for the two processes, respectively. If the fracture region behaves like an equivalent porous medium, the system has the following stable properties: 1) specific discharge is uniform in any direction and can be predicted from a permeability tensor and, 2) hydraulic effective porosity is directionally stable. Fracture systems sith two parallel sets of continuous fractures satisfy criterion 1 . However, in these systems hydrsulic effective porosity is directionally dependent, and thus, criterion 2 is violated. Thus, for some fracture systems. fluid flow can be predicted using porous media assumptions, but it may not be possible to predict transport using porous media assumptions.
\end{abstract}

Two discontinuous fracture systems were studied which satisfied both criteri3. Hydraulic 
effective porosity for both systems has a value between rock effective porosity and total porosity. A length-den .:y analysis (LDS) of Canadian fracture data shows that porous media equivalence for fluid flow and transport is likely when systems have narrow aperture distributions. Hydraulic effective porosities are equal to and greater than rock effective porosity for the continuous and the jiscontinuous systems exhibiting pornus media equivalence in the LDS, respectively. Ail fracture systems studied showed different polar plots of longitudinal geometric dispersivity. In most porous media transport studies, anisotropic media is treated as equivalent isotropic media such that longitudinal geometric dispersivity is directionally stable. The use of directionally-stable longitudinal geometric dispersivities for these fracture systems could lead to serious errors in transport prediction. 
Table of Contents

Table of Contents ….................................................................................................. i

List of Figures ............................................................................................................. iv

List of Tables .......................................................................................................... ix

Notation ................................................................................................................

Acknowledgments ...........................................................................................................

CHAPTER 1 INTRODUCTION …….............................................................

CHAPTER 2 LITERATURE REVIEW

2.1 INTRODUCTION ..........................................................................................

2.2 DISPERSION IN POROUS MEDIA ……........................................................... 3

2.2.1 The Advective Transpon Process ...................................................................... 4

2.2.2 The Dispersive Transpon Process .................................................................... 5

2.2.3 Stochastic Models for Porous Media Dispersion ............................................ 10

2.3 DISCRETE MECHANICAL TRANSPORT MODELS FOR

FRACTURE ROCK MASSES ………............................................................... 15

\section{CHAPTER 3 THEORETICAL DEVELOPMENT}

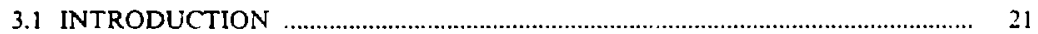

3.2 MICROSCOPIC LEVEL TRANSPORT ………………….................................. 22

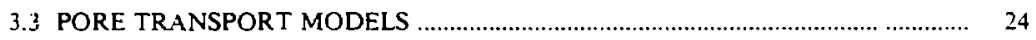

3.4 POROUS MEDIA TRANSPORT MODEL ………….......................................... 27

3.5 PROPER FLOW FIELD AND APPROPRIATE TEST

SECTION USED IN A TRACER EXPERIMENT ……........................................ 33

3.6 EXECUTING THE SET OF TRACER EXPERIMENTS TO

MEASURE DIRECTIONAL MECHANICAL TRANSPORT … ........................... 35

3.7 MEASURING MECHA.VICAL TRANSPORT WITH THE

BREAKTHROUGH CLRVE ....................................................................... 38

3.7.1 Mean of the Breakthrough Curve .................................................................. 38

3.7.2 Variance of the Breakthrough Curve .................................................................. $4 \mathrm{I}$

3.8 EQUIVALENT POROUS MEDIUM BEHAVIOR …........................................ 47 
CHAPTER 4 THE DISCRETE NUMERICAL MODEL

4.1 INTRODUCTION ..................................................................................................... 55

4.2 FRACTURE SYSTEM GENERATION STAGE ................................................ 55

4.3 HYDRAULIC HEAD CALCULATION .............................................................. 58

4.4 MECHANICAL TRANSPORT SIMULATION ……................................................ 60

CHAPTER 5 INVESTIGATION OF CONTINUOUS FRACTLRE SYS1 EMS

5.1 INTRODUCTION

5.2 CONTINUOUS SYSTEM WITH TWO SETS OF CONSTANT-APERTURE FRACTURES ........................................................... 69

5.3 SYSTEM WITH TWO ORTHOGONAL SETS

OF CONTINUOUS FRACTURES

CHAPTER 6 INVESTIGATION OF DISCONTINUOUS FRACTURE SYSTEMS

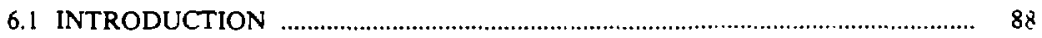

6.2 DISCONTINUOUS FRACTURE SYSTEM OF TWO SETS

ORIENTED AT $0^{\circ}$ AND $30^{\circ}$

6.3 DISCONTINUOUS FRACTURE SYSTEM OF TWO SETS

ORIENTED AT $0^{\circ}$ AND $60^{\circ}$

6.4 SENSITIVITY ANALYSIS

6.4.1 Sensitivity of Mean Geometric Parameters

and Set Areal Density

6.4.2 Sensitivity of Distributed Geometric Parameters

\section{CHAPTER 7 INVESTIGATION OF MECHANICAL TRANSPORT AT} RESEARCH SITE IN MANITOBA, CANADA

7.1 INTRODUCTION

7.2 CONSTANT APERTURE LENGTH-DENSITY SERIES

7.3 DISTRIBUTED APERTLRE LENGTH-DENSITY SERIES

73.1 Distributed Aperture Length-Density Study with

Standard Deviation Equal to Mean Aperture

7.3.2 Distributed Aperure Length-Density Study with 


\section{CHAPTER 8 CONCLUSIONS AND RECOMMENDATIONS}

8.1 CONCLUSION ............................................................................................... 191

8.2 RECOMMENDATIONS ................................................................................... 197

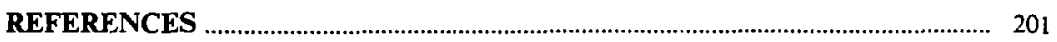




\section{List of Figures}

Figure 2-1 Three Microscopic Mechanisms in Mechanical Transport.

Figure 2-2 Graphical Representation of Mixing Conditions at a Node.

Figure 2-3 Modeling Conditions Used in Earlier Mechanical Transport Models for Fracture Networks.

Figure 3-1 Modeling of Transport Within a Control Vollime at the Microscopic Level.

Figure 3-2 Early Stages of Transpor When a Uniform Pulse is injected Between Two Parallel Plates Under Laminar Slow Conditions.

Figure 3-3 Tortuosity for a Single Pore.

Figure 3-4 Determination of the Fow Field for an Anisotropic Porous Medium With a Constant Hydraulic Gradient Using Fow Net Theory.

Figure 3-5 Example of Groundwater Flow in an Anisotropic Porous Medium Showing a Cross-Hatched Zone Where Travel Length is Constant.

Figure 3-6 Procedure Used in Conducting a Set of Tracer Experiments to Measure Directional Mechanical Transport for an Anisotropic Porous Medium.

Figure 3.7 Divergence of Flow Paths in Flow Regions Within an Inhomogeneous Regional Flow System.

Figure 3-8 Semilog Plot of Peclet Number Versus the Ratio of the Variance of the Breakthrough Curve to the Squared Mean Travel Time.

Figure 3-9 Three Breakthrough Curves for a) Pe of 0.5. b) Pe of 0.2, and c) Pe of 0.025 .

Figure 3-IC Square Root of Permeability in Direction of Flow for the Annisotropic Porous Medium Shown in Figure 3-4.

Figure 4-1 Stochastic Generation of a Fracture System Consisting of Two Sets of Fractures.

Figure 42 A Typical Line Element of Aperture is $W$ ith Labeled Nodes.

Figure 4-3 Redistribution of Streamtu'es at a Node.

Figure 4-4 Creation of New Streamtubes at a Node.

Figure 4j Fracture Network With Inflow Streamtubes Initiated in Elements 1.2. and 5.

Figure 5-1 Fracture System With Two Sets of Parallel. Continuous, and Constant Aperture Fractures.

Figure 5-2 Convergence of Specific Discharge and Porosity to Their Theoretical Values as Size of Flow Region Increases (Ratio is $\mathrm{q} ; \mathrm{q}_{\mathrm{T}}$ or $\phi / \phi_{\mathrm{T}}$ ).

Figure 5-3 Polar Plots of a) Specific Discharge and b) Average Linear Velocity Factor Versus Direction of Flow for System of Tivo Set of : arallel. Continuous. and Constant Aperure Fractures.

Figure 5-4 Polar Plot of Hydraulic Effective Porosity Versus Direction of Flow for System of Two Sets of Paralle!. Continuous. and Constant Aperture Fractures. 
Figure 5-5 Tortuosity Versus Direction of Flow for Fracture System of Two Sets of Parallel, Continuous, and Constant Aperture Fractures.

Figure 5-6 Actual Mean Pore Velocity, Calculated Mean Pore Velocity, and Calculated Mean Pore Velocity With Tortuosity of $\sqrt{2}$ for Fracture System of Two Sets of Continuous, Parallel, and Constant Aperture Fractures.

Figure 5-7 Polar Plot of Longitudinal Geometric Dispersivity for Fracture System of Two Sets of Continuous, Parallel, and Constant Aperture Fractures.

Figure 5-8 Fracture System With Two Orthogonal Sets of Continuous Fractures.

Figure 5-9 Polar Plots of a) Specific Discharge and b) A verage Linear Factors Versus Direction of Flow for System With Two Orthngonal Sets of Continuous Fractures.

Figure 5-10 Polar Plot of Hydraulic Effective Porosity for System of Two Orthogonal Sets of Continuous Fractures.

Figure 5-11 Polar Plot of Tortuosity for Fracture System With Two Orthogonal Sets of Continuous Fractures.

Figure 5-12 Polar Plot of Longitudinal Geometric Dispersivity for System With Two Orthogonal Sets of Continuous Fractures.

Figure 6-I Fracture Network in the Generation Region for Discontinuous Fracture System of Two Sets of Fractures Oriented at $0^{\circ}$ and $30^{\circ}$ With Constant Aperture and Length.

Figure 6-2 Networks of a) Fractures and b) Connected Fracture Segments in Flow Region Oriented at $130^{\circ}$ for Discontinuous Fracture System of Two Sets Driented at $0^{\circ}$ and $30^{\circ}$.

Figure 6-3 Polar Plot of Syuare Root of Permeability in Direction of Flow for Discontinuous Fracture System of Two Sets Oriented at $0^{\circ}$ and $30^{\circ}$.

Figure 6-4 Mean DEVF and Mean DEVA Versus Direction of Flow for Discontinuous Fracture System of Two Sets Oriented at $0^{\circ}$ and $30^{\circ}$.

Figure 6-5 Polar Plot of Tortuosity for Discontinuous Fracture System of Two Sets Oriented at $0^{\circ}$ and $30^{\circ}$.

Figure 6-6 Actual Mean Pore Velocity and Calculated Mean Pore Velocity for First Reaiization of Discontinuous Fracture System of Two Sets Oriented at $0^{\circ}$ and $30^{\circ}$.

Figure 6-7 Polar Plots of Total Porosity. Hydraulic Effective Porosity. and Rock Effective Porosity for Discontinuous Fracture System of Two Sets Oriented at $0^{\circ}$ and $30^{\circ}$.

Figure 6-8 Three Breakthrough Curves for Directions of Flow Which Increase From the Direction of Maximum Permeability to the Diection of Minimum as One Proceeds Down the Figure.

Figure 6-9 Polar Plot of Longitudinal Geometric Dispersivity Versus Direction of Flow for Discontinuous System of Two Sets Oriented at $0^{\circ}$ and $30^{\circ}$.

Figure 6-10 Fracture Network in the Generation Region for Discontinuous System of Two Sets Oriented at $0^{\circ}$ and $60^{\circ}$.

Figure 6-11 Networks of a) Fractures and b) Connected Fraziure Segnents in the Flow 
Region Oriented at $30^{\circ}$ for Discontinucus System of Two Sets Orienied at $0^{\circ}$ and $60^{\circ}$.

Figure 6-12 Mean Hydraulic Effective Porosity for Orientations $30^{\circ}, 75^{\circ}$, and $120^{\circ}$ Versus the Number of Realizations.

Figure 6-13 Polar Plot of Square Rnot of Permeability in Direction of Flow for Discontinuous System of Two Sets Oriented at $0^{\circ}$ and $60^{\circ}$.

Figure 6-14 Mean DEVF and Mean DEVA Versus Direction of Flow fror Discontinuous System of Two Sets Oriented at $0^{\circ}$ and $60^{\circ}$.

Figure 6-15 Polar Plot of Tortuosity for Discontinuous System of Two Seis Oriented at $0^{\circ}$ and $60^{\circ}$.

Figure 6-16 Polar Plots of Rock Effective Porosity, Hydraulic Effective Porosity, and Total Porosity for Discontinuous $S y s t e m$ of Two Sets Oriented at $0^{\circ}$ and $60^{\circ}$.

Figure 6-17 Polar Plot of Longitudinal Geometric Dispersivity for Discontinuous System of Two Sets Oriented at $0^{\circ}$ and $60^{\circ}$.

Figure 6-18 Longitudinal Geometric Dispersivity Versus Width of Flow Region for Discontinuous System of Two Setis Onented at $0^{\circ}$ and $60^{\circ}$.

Figure 6-19 Three General Types of Sensitivity Relationships.

Figure 6-20 Total Porosity, Rock Effective Porosity, and Hydraulic Effective Porosity Versus Numbcr of Fractures in Set, Mean Fracture Length, and Orientation of Set 2.

Figure 6-21 Specific Discharge Versus Number of Fractures in Set, Nisan Fracture Length, and Orientation of Set 2.

Figure 6-22 Rock Effective Porosity and Specific Discharge Versus Connertivity.

Figure 6-23 Mean Travel Time Versus Number of Fractures in Set. Mean Fracture Length, and Orientation of Set 2.

Figure 6-24 Variance of the Breakthrough Curve Versus Number of Fractures in Set. Mean Fracture Length. and Orientation of Set 2.

Figure 7-1 Map of Hydrogeological Research Site in Manitoba. Canada.

Figure 7-2 Fracture Network in the Generation Region for Discontinuous System With Mean Fracture Length of $10 \mathrm{~m}$.

Figure 7-3 Network of a) Fractures and b) Connected Fracture Segments in the Flow Region Oriented at $0^{\circ}$ for Discontinuous System With Mean Fracture Length of $10 \mathrm{~m}$.

Figure 7-4 Fracture Network in the Generation Region for Discontinuous System With Mean Fracture Length of $35 \mathrm{~m}$.

Figure 7.5 Network of a) Fractures and b) Connected Fracture Segments in the Fow Region Oriented at $0^{\circ}$ for Discontinuous System with Mean Fracture Length of $35 \mathrm{~m}$.

Figure 7-6 Polar Plots of Turtuosity for a) Systems With Mean Fracture Lengths of 30 and $35 \mathrm{~m}$ and b) System With Mears Fracture Length of $50 \mathrm{~m}$ and the Continuous 
Fracture System.

Figure 7-7 Polar Plots 0: Hydraulic Effective Porosity for a) Systems With Mean Fracture Lengths of 30 and $35 \mathrm{~m}$ and b) System With Mean Fracture Length of $57 \mathrm{~m}$ and the Continuous Fracture System.

Figure 7-8 Polar Plots of Longitudinal Geometric Dispersivity for Systems With Mean Fracture Lengths of a) $30 \mathrm{ra}$ and b) $35 \mathrm{~m}$.

Figure 7-9 Polar Plots of Longutudinal Geometric Dispersivity for a) System With Mean Fracture Length of $50 \mathrm{~m}$ and $\mathrm{b}$ ) the Continuous Fracture System.

Figure 7-10 Polar Plot of Mean Longitudinal Geometric Dispersivity for Five Realizations of the Sysiem With Mean Fracture Length of $50 \mathrm{~m}$.

Figure 7-1: Polar Hlot of Hydraulic Effective Porosity for System With Mean Fracture Length of $50 \mathrm{~m}$ Using Square Flow Region of Width $330 \mathrm{~m}$.

Figure 7-12 Polar Flot of Hydraulic Effective Porosity for System With Mean Fracture Length of $50 \mathrm{~m}$ Using Square Flow Region of Width $17.5 \mathrm{~m}$.

Figure 7-13 Poiar Plot of Hydraulic Effectiv: Porosity for System With Mean Fracture Length of $50 \mathrm{~m}$ Using Square Flow Region of Width $60 \mathrm{~m}$.

Figure 7-14 Fracture Network in the Generation Region for Continuous System in the First Distributed Aperture Study.

Figure 7-15 Mean Hydraulic Effective Porosity in Al: Directions Versus the Number of Realizations.

Figure 7-16 Standard Error in Mean Hydraulic Effective Porosity Versus the Number of Realizations.

Figure 7-17 Fracture Network in a) Generation Region and b) Flow Region Oriented at $60^{\circ}$ for Realization 12 of the Continuous System in the First Distributed Aperture Study.

Figure 7-18 Breakthrough Curve for Direction of Flow $120^{\circ}$ for Continuous System in the First Distributed Apeiture Study,

Figure 7-19 Polar Plots of Mean Hydraulic Effective Porosity After Five Realizations and Ten Realizations.

Figure 7-20 Polar Plots of Mean Hydraulic Effective Porcsity a fte; F fteen Realizations and Twenty-Five Realizations.

Figure $7-21 \quad$ Mean DEVF and Mean DEV $t$ Versus Direction of Flow for Continuous System in the First Distributed Aperture Study.

Figure 7-22 Polar Plot of Mean Tortuosity for Continuous System in the First Distributed Aperture Stuay.

Figure 7-23 Polar Plots of Total Porosity, Hydraulic Effective Porosity, and Rock Effective Porosity for Discontinuous System With Mean Fracture Length of $50 \mathrm{~m}$ and Linearly Correlated Apcrtures.

Figure 7-24 Breakthrough Curve in a Direction in Whica Hydraulic Effective Porosity is Three Times Larger Than Rock Fffective Forosity: 
Figure 7-25 Mean DEVF and Mean DEVA Versus Direction of Flow for System Witu Mean Fracture Length of $50 \mathrm{~m}$ and Linearly Correlated Apertu:es.

Figure 7-26 Polar Plot of Mean Tortuosity for System With Mean Fracture Length of $\mathbf{5 0} \mathrm{m}$ and Linearly Correlated Apertures.

Figure 7-27 Polar Plot of Mean Hydraulic Efrective Porosity for System With Mea:1 Fracture Length of $50 \mathrm{~m}$ in the First Distributed ipertlire Study.

Figure 7-28 Mean DEVF and Mean DEVA Versus Direction of Flow for System With Mean Fracture Length of $50 \mathrm{~m}$ in the First Disıributed Aperture Study.

Figure 7-29 Polar Plots of Total Porosity and Hydraulic Effective Porosity for Continuous System With $\sigma_{b} / \mu_{b}$ of 0.3 .

Figure 7-30 IVean DEVF and Mean DEVA Versus Direction of Flow for Continuous System With $\sigma_{b} / \mu_{b}$ of 0.3 .

Figure 7-31 Polar Plot of Tortuosity for Continuous Systern With $\sigma_{\mathrm{b}} / \mu_{\mathrm{b}}$ of 0.3 .

Figure 7-32 Polar Plot of Longitudinal Geometric Dispersivity for Continuous System With $\sigma_{\mathrm{b}} / \mu_{\mathrm{b}}$ of 0.3 .

Figure 7-33 Polar Plots of Total Porosity, Rock Effective Porosit, and Hydraulic Effective Porosity for Discontinuous System With $\mu_{1}$ of $50 \mathrm{~m}$ anc $\sigma_{b} / \mu_{b}$ of 0.3 .

Figure 7-34 Mean DEVF and Mean DEVA Versus Di ection of Flow for Discontinuous System With $\mu_{1}$ of $50 \mathrm{~m}$ and $\sigma_{\mathrm{b}} / \mu_{\mathrm{b}}$ of 0.3 .

Figure 7.35 Polar Plot of Longitudinal Geometric Dispersivity for Discontinuous System with $\mu_{1}$ of $S 0 \mathrm{~m}$ and $\sigma_{\mathrm{b}} / \mu_{\mathrm{b}}$ of 0.3 .

Figure 7-36 Polar Plot of Toruosity for Discontinuous System $W_{i t h} \mu_{1}$ of $50 \mathrm{~m}$ and $\sigma_{b} / \mu_{b}$ of 0.3 . 


\section{List of Tables}

Table 41 Macroscopic Parameters Calculated in Mechanical Transport Simulation Stage.

Table 5-I Specific Discharge Results for Fracture System With Two Sets oi Parallel, Continuous, and Constant-Aperture Fractures.

Table 5-2 Specific Discharge Results for Fracture System With Two Orthogonal Sets of Fractures.

Table 6-1 Orientariens, Estimia: d Directions of Flow, and Flow Region Sizes Used in Monte Carlo Simulation of Discontinuous System Consisting of Two Sets of Fractures Oriented at $0^{\circ}$ and $30^{\circ}$.

Table 6-2 Orientations, and Estimated Directions of Flow Used in Monte Carlu Simulation of the Discontinuous System Consisting of Two Sets of Fractures Oriented at $0^{\circ}$ and $60^{\circ}$.

Table 6-3 Orientations and Directions of Flow Calculated for Monte Carlo Study of the Discontinuous System of Two Sets Oriented at $9^{\circ}$ and $60^{\circ}$.

Table 6-4 Mean Sensitivity and Maximum Magnitude of Relative Sensitivity for Mean Orientation, Set Areal Density, and Mean Fracture Length Sensitivity Studies. 


\section{Notation}

A

ANFC angle of flow based on uniformity of flow, degrees

ANFD angle of flow based on components of specific discharge, degrees

b fracture aperture, $L$

c concentration, $\mathrm{M} / \mathrm{L}^{3}$

(C) conductance matrix, L/T

$c_{0} \quad$ concentration in an outflow fracture, $M / L^{3}$

$\overrightarrow{\mathrm{d}} \quad$ diffusive mass flux, $\mathrm{M} / \mathrm{TL}^{2}$

$d_{c} \quad$ diameter of capillary tube, $\mathrm{L}$

D free solution molecular diffusion coefficient, $\mathrm{L}^{2} / \mathrm{T}$

DEVA deviation in angle of flow, degrees

DEVF deviation in flow

$\left.\mid D_{F}\right] \quad$ dispersed plug flow dispersion tensor, $L^{2} / T$

$D_{L} \quad$ axial dispersed plug flow longitudinal dispersion coefficient, $L^{2} / T$

Do effective molecular diffusion coefficıent $L^{2} / T$

E normalized concentraiion, I/T

$E^{\prime} \quad$ dimensionless concentration

Es dimensionless concentration for zone of slow movement

$E_{F}$ dimensicnless concentration for zone of fast movement

B acceleration of gravity. $L / T^{2}$

h half width of parallel plate conduit. L 
j hyčraulic gradient

[k] intrinsic permeability tensor, $\mathrm{L}^{2}$

[K] permeability tensor, $L / T$

$K_{f}$ permeability in direction of flow, $\mathrm{L} / \mathrm{T}$

$\mathbf{K}_{\mathbf{x}} \quad$ maximum principal permeability, L/T

$\mathrm{K}_{\mathrm{y}} \quad$ minimum principal permeability, $\mathrm{L} / \mathrm{T}$

1 fracture length, $\mathrm{L}$

L linear length of travel, $\mathrm{L}$

L. mean travel length of flow between sides 2 and $4, L$

L $\quad$ width on side 2 of zone of continuous flow from side 2 to $4, \mathrm{~L}$

$L_{e} \quad$ length of element, $L$

LNC width on side 2 of zone of non-continuous flow frem side 2 to $4, L$

Lst total travel length of streamtube, $L$

L width of flow region, $L$

Ly height of flow region, $L$

$\vec{m}$ mechanical dispersive flux, $M / T L^{2}$

[M] mechanical dispersion tensor, $\mathrm{L}^{2} / \mathrm{T}$

$M_{L} \quad$ longitudinal mechanical dispersion coefficient, $L^{2} / T$

MPV mean pore velocity, $\mathrm{L} / \mathrm{T}$

NC number of streamtubes flowing between sides 2 and 4

- fracture orientation, degrees

p pressure, $\mathrm{M} / \mathrm{LT}^{2}$

Pe Peclet number 
specific discharge, $\mathrm{L} / \mathrm{T}$

$\mathrm{q}_{\mathrm{T}}$ theoretical magnitude of specific discharge, $\mathrm{L} / \mathrm{T}$

Q flow rate in direction of flow, $L^{2} / T(2-D)$ or $L^{3} / T(3-D)$

Qc flow rate for fluid that flows from side 2 to $4, \mathrm{~L}^{2} / \mathrm{T}$

$Q_{e} \quad$ flow rate in element, $L^{2} / T$

QN total flow rate into node, $I^{2} / T$

QNe flow rate for fluid flowing into side 2 that does not exit on side $4, L^{2} / T$

QsT flow rate in streamtube, $\mathrm{L}^{2} / \mathrm{T}$

Q $\quad$ flow rate in the $x$ direction, $L^{2} / T(2-D)$ or $L^{3} / T$ (3-D)

QSI flow rate into side $1, \mathrm{~L}^{2} / \mathrm{T}$

Q52 flcw rate into side $2, \mathrm{~L}^{2} / \mathrm{T}$

QS3 flow rate out of side 3, $\mathrm{L}^{2} / \mathrm{T}$

QS4 flow rate out of side $4, L^{2} / T$

$r \quad$ ccordinatc perpendicular to direction of flow, $L$

$r_{b} \quad$ hydraulic radius, $L$

s coordinate in direction of flow, $\mathrm{L}$

sf shape factor

S relative sensitivity

SD standard deviation of normalized breakthrough curve, T

$\mathrm{S}_{\mathfrak{m}} \quad$ mear. sensitivity

S, specific surface, $1 / \mathrm{L}$

1 time, $\mathrm{T}$

$T \quad$ mean jlow travel time. $T$ 
IsT travel time of streamtube in element, $T$

TM mean of normalized breakthrough curve, $\mathrm{T}$

u fracture velocity, I/T

U maximun velocity in parallel plate conduit, $\mathrm{L} / \mathrm{T}$

$v \quad$ velocity, $\mathrm{L} / \mathrm{T}$

$\bar{v} \quad$ average cross sectional velocity, $\mathrm{L} / \mathrm{T}$

$\mathrm{V}$ total volume, $\mathrm{L}^{3}$

$\mathrm{V}_{\mathrm{c}} \quad$ total conductive void volume, $\mathrm{L}^{3}$

VLIN average linear velocity, $\mathrm{L} / \mathrm{T}$

VPORE calculated mean pore velocity, $L / T$

[a] genmetric dispersivity tensor, $\mathrm{L}$

$\alpha_{3} \quad$ longitudinal geometric dispersivity for isotropic medium, L

$\alpha_{\mathrm{T}}$ longitudinal geometric dispersivity, $\mathrm{L}$

$a_{l} \quad$ transverse geometric dispersivity for isotropic medium. L

$5 \quad$ angle between scanline and fracture pole. degrees

$\eta \quad$ ordinate for coordinate system inside fracture. $\mathrm{L}$

$\theta \quad$ angle of flow, degrees

$\theta_{\mathrm{T}} \quad$ theoretical angle of flow, degrees

$\lambda_{1} \quad$ linear fracture density, $1 / \mathrm{L}$

$\lambda_{A} \quad$ set areal density, $1 / \mathrm{L}^{2}$

$\mu \quad$ fluid viscosity, M/LT

$\mu_{i} \quad$ mean of $\mathrm{i}$

$\xi \quad$ abscissa for coordinate system inside fracture, $L$ 


$\begin{array}{ll}\rho & \text { fluid density, } \mathrm{M} / \mathrm{L}^{3} \\ \sigma_{\mathrm{i}} & \text { standard deviation of } \mathrm{i} \\ \sigma^{2} & \text { variance of normalized breakthrough curve, } \mathrm{T}^{2} \\ \tau & \text { tortuosity } \\ \phi^{2} & \text { total porosity } \\ \phi_{\mathrm{H}} & \text { hydraulic effective porosity } \\ \phi_{\mathrm{R}} & \text { rock effective porosity } \\ \phi_{\mathrm{T}} & \text { theoretical total porosity } \\ \Phi & \text { hydraulic head, L }\end{array}$




\section{Acknowledgments}

Acknowledgment is due to the U. S. Department of Energy through the Office of Crystalline Repository Development (OCRD) for support of this study under contract number DEAC03-7CSFO0098. The assistance from Bill Ubbes and A. Berge Gureghian of OCRD is gratefully acknowledged.

I should like to express my appreciation to my major field advisor Paul Witherspoon for his guidance and counsel in many aspects of my research. Many sincere thanks are also due to Charles Wilson, Jane Long, and Kenzi Karasaki for their helpful suggestions and advice. 


\section{CHAPTER 1 \\ INTRODUCTION}

Concerns about radioactive waste storage and the injection of toxic pollutants deep underground have focused interest on the problems of fluid flow and mass transport in groundwater systems. The disposal of pollutants in or near a rock mass where fractures constitute the major conduits of groundwater movement is a central problem. The primary objective of this research is to determine when a fractured rock mass may be treated as an equivalent porous redium for transport studies.

In porous media, the size, shape, and degree of interconnection of the intergranular pores regulate fluid flow and transport. The void region is well connected and the number of pores per volume of porous medium is very large, so that the medium may be treated as a continuum in which macrosce sic fluid flow and transport properties are considered without regard for the actual movement of individual fluid particles. The number of connected fractures per volume of rock is much less in a fractured rock mass than in a porous medium: therefore, a larger ssimple of rock may be required before the porous medium approach is applicable. When the porous medium approach is not appropriate, a discrete model which simulates iransport in each fracture of the network must be used. The discrete approach requires detailed information on the geometry of the fracture system and hence may tequire an excessive amount of data and computational effort. Thus, the continuum approach is preferat?e if it can be shown to be aypropriate.

in order to evaluate whether the continuum approach is applicable, one must demonstrate that the fracture system has the same transport behavior as that of an equivalent porous medium. However, fracture systems may be anisotropic and transport in anisotropic media is not fully understood. The reason is that no solution is available to determine the components of the dispersivity tensor in an anisotropic medium (Freeze and Cherry, 1979, pp. 552).

However, mechanical transpor, the component of transport that is due to the movement 
of fluid through the conductive flow channels, can be evaluated for anisotropic media. Thus, the conditions under which fracture networks behave like equivalent porous media can be investigated by focusing on mechanical transport.

The directional variations of the ratio of fluid flux to mean velocity, tortuosity, and longitudinal mecharical dispersion must be understood to evaluate mechanical transport. In an anisotropic medium, the ratio of flux to mean velocity is assumed to be independent of direction of flow and equal to porosity. Thus, a test for equivalent porous medium behavior is to determine if the ratio of flux to velocity is constant in all directions. Less is understood about the directional dependence of tortuosity and mechanical dispersion in anisntropic porous media. The prevailing practice is to treat an anisotropic porous medium as an equivalent isotropic medium in transport studies, implying that each transport parameter has no directional dependence. This work introduces the concepi that longitudinal mechanical transport and tortuosity are both dependent on the direction of flow. Thus, the evaluation of these parameters in anisotropic fracture networks will lead to a better understanding of the transport phenomenon in all permeable media.

The fact that fluid flow and mechanical transport are coupled processes makes it necessary to also investigate equivalent porous medium behavior for fluid flow. The directional properties of specific discharge are used to investigate equivalent continuum behavior for fluid flow. When the fracture system exhibits continuum behavior, specific discharge can be predicted in any direction from a permieability tensor.

A numerical model is used in this research to simulate mechanical transport in discrete fracture networks. We assume that fluid flow is restricted to planar fractures within an impermeable rock matrix and that mechanical transport is the only transport process. The simulation of mechanical transport uses a new streamtubing technique which traces the detailed movement of fluid within streamtubes from inflow to outflow boundaries along the fracture system. 


\section{CHAPTER 2 \\ LITERATURE REVIEW}

\subsection{INTRODUCTION}

Tracer injected into a groundwater aquifer will not migrate downstream retaining its shape, but will spread and mix with the ambient fluid. Dispersion of the tracer is caused by microscopic mixing occurring within the pores of the medium. This literature review will: 1) introduce the transpcrn processes and parameters used to model dispersion in porous media; 2) present key areas of research in porous media transport and; 3) review mechanical transport models for fractured rock masses.

The nrimary objective of this research is to determine when a fractured rock mass can be treated like an cquivalent porous medium. Porous media transport modeling is reviewed so that continuum parameters characterizing transport can be evaluated. Areas of uncertainty where research is needed are also discussed. Two specific problem areas, applicability of the Fickian dispersive approach and dispersion in anisotropic media, will be investigated.

Three earlier discrete mechanical transport mociels are discussed below. The modeling techniques and principles used in each model are reviewed in detail. A new model which simulates mechanical transport in fractured rock masses is developed in Chapter 4. This new model is based on the physics of fluid flow to provide a sound and realistic simulation of mechanical transport.

\subsection{DISPERSION IN POROUS MEDIA}

The porous media dispersion model consists of an advective transport precess coupled with a dispersive iransport process. The advective process accounts for transport by the mean motion of flow. Advective transport alone causes no distortion in the shape of a pollutant plume as poilutant is merely transported with the mean flow. The dispersive process allows for the spread of a pollutant resulting from complex microscopic mixing that occurs within the pores. In the next two sections.modeling of the advective and the dispersive processes are 
discussed. Section 2.2.3 reviews the fairly recent approach of stochastic transport modeling for porous media. A numerical stochastic model of mechanical transpor will be developed in Chapter 4.

\subsubsection{The Advective Transport Process}

In the advective process, a pollutant moves with the mean flow velocity. In porous media modeling, the mean rate of advection is expressed in terms of the quantity of fluid flowing through the medium because fluid flow paramete:s are generally much easier to determine than transport parameters. The hydraulic effective porosity is used in this study to express the relationship between fluid flow and transport, and is defined as the specific discharge divided by the average linear velocity. The average linear velocity is the ratio of the straight or linear travel length to the mean flow travel time. Considerable laboratory work has been carried out to determine the relationship between hydraulic effective porosit; and total porosity. Von Rosenbury (1956) conducted tracer experiments in packed columns of Ottawa sand and found that for this homogeneous and isotropic medium the hydraulic effective porosity was nearly equal to the total forosity. Biggar and Nielsen (1960) obtained mixed results when they investigated a set of four porous media: glass beads, Oakley sand, Columbia silt loam, and Irolo loam. In the case of the glass beads, the total porosity provided a good estimate of the hydraulic effective porosity. However, the hydraulic effective pornsity was less than the porosity for the three other porous media. Biggar and Nielsen (1960) atiributed this deviation to the presence of stagnant void regions. In contrast to the findings of Biggar and Nielsen. Ellis et al. (1968) found that, in laboratory tracer experiments involving packed sand coiumns, the hydraulic effective porosity was generally larger than the porosity. The hydraulic effective porosity may be greater than the porosity if microscopic regions of slow movement exist which have a minor influence on the total flow rate passing through the medium. but have a major influence on the travel time for particles flowing into these zones. The slow movement in these zones produces a very large mean flow travel time which can result in the hydiaulic effective porosity being larger than the porosity. 
Well testing methods provide a means of evaluating the hydraulic effective porosity in the field. Hazzaa et al. $(1965,1966)$ applied the two-well pulse method to predict the hydraulic effective parosity for an inhomogeseous aquifer of sand and gravel inierbedded with clay lenses. Hazzaa et al. in a series of tests concluded that the hydraulic effective porosity was less than the porosity.

Grove and Beetem (1971) used an innovative recharging-discharging well technique to determine the hydraulic effective porosity for a fractured carbonate aquifer of Culebra dolomite. In this technique, water is pumped from one well and injected into another well at the same flow rate until a steady state hycraulic head distribution is established in the aquifer. Tracer is then introduced into the recharging well, and the breakthrough curve measured at the discharging well. The hydraulic effective porosity was found to be within the range of the estimated porosity.

The standard multiple well method of jijecting tuacer into a recharging well and monitoring concentration at strategically located observation wells was employed by Hoehn and Roberts (1982). The multiple-well test was conducted in a vertically homogeneous, poorlysorted alluvial gravel aquifer. It was concluded that the hydraulic effective porosity was less than the porosity.

Thus, both field and laboratory tests have demonstrated that the hydraulic effective porosity $\left(\phi_{\mathrm{H}}\right)$ is not necessarily equal to the total porosity $(\phi)$. Experimental evidence rrom the laboratory indicates that the relationship between $\phi_{H}$ and $\phi$ is dependent on the type of porous medium. Where there is a vell-ordered pore structure (i.e. glass beads), $\phi_{\mathrm{H}}$ is approximately equal to $\phi$. However, where the pore structure is irregular and nonuniform, the hydraulic effective porosity differs from the total porosity and is usually less than $\phi$ becausc of the presence of stagnant void regions.

\subsubsection{The Dispersive Process}

The distortion in the shape of a contaminant plume for a conservative pollutant is caused by the interaction of molecular diffusion and mechanical dispersicin. Molecular diffusion is the 
mixing caused by the kinetic energy of randonly moving solute particles. Mecharucal dispersion is the mixing induced by the movement of fluid thiough the conductive channels of the medium. The principle microscopic mechanisms of mechanical jispersion, as illustrated in Figure 2-1, are the velocity distribution across a flow channel which causes particles to move faster in the conter than along the sides of the channel; the flow rate variation from one channel to another which dictates the direction particles will travel; and the geometry of the pores which causes particles to travel along tortuous paths. Molecular diffusion conirols dispersion when the flow velocity is very low; mechanical dispersion rapidly becomes the governing mode of transport as flow velocity increases.

Presently, the classical approach is $\rightarrow$ idely usec to characterize the dispersive process because of the simplicity of this approach, and because this approach has been used with moderate success in analyzuig laboratory tracer experiments. The classical appioach was formulated from Fick's law for molecular diffusion. In this apprcach. the mass flux dispersing actoss a unit area is equal to the product of a second-order dispersion tensor consisting of tonstant coefficients and the negative of the concentration gradient. Von Rosenburg (1956) presented qualitative justification for applying the classical approach to porous media dispersion based on Tayior's analysis (1953) of dispersion in a pipe. Taylor demonstrated that after an initial period, the Fickian approach may be used to model longitudınal jispersion in a pipe.

The laboratory tracer experiments that confirmed the clas' zal dispersive approach were generally conducted using packed columns of isotropic, homogeneous porous media. A porous medium sample was carefully placed into an open-ended cylinder and constant hydraulic head boundary conditions were applied across she inlet ano the outlet of the packed column to create a uniform flow field. A consistent uniformi flow field throughout the sample was required to evaluate meaningful transport parameters from the tracer experiment. Tracer was then introduced at the inlet to the column and concentration was measured at various points within the column or at the outlet. Perkins and Johnston (1963) present a comprehensive review of laboratory dispersion studies that were based on this classical approach. 


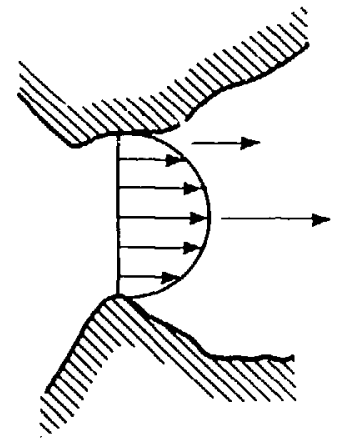

Velocity distribution in flow channel
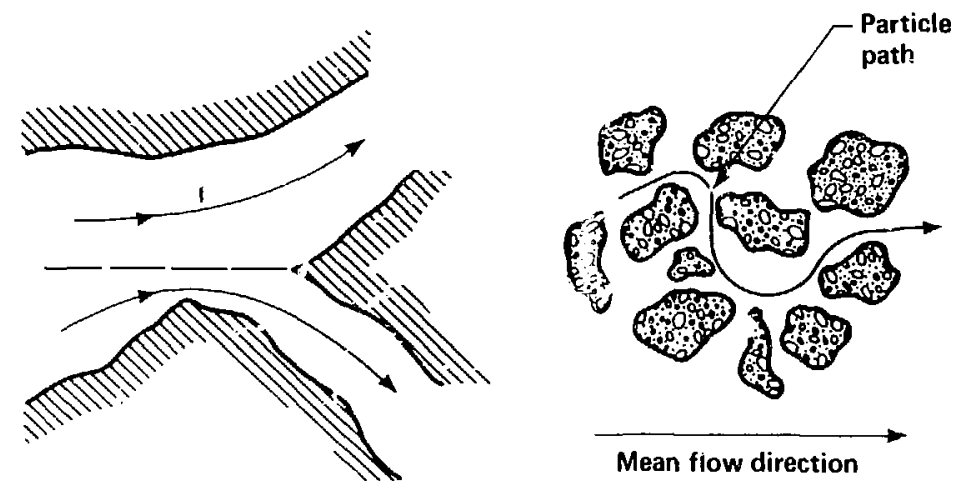

Flow variatıon

XEL 827.7177

Figure 2-1 Three Microsco $\cdots$ Mechanisms in Mechanical Transport. 
Zispersion studies have also been conducted in the field. Two general techniques are used to evaluate dispersion from field measurements. In the first technique, tracer tests are conducted and concentration measurements are used to evaluate dispersion coefficients. A multitude of different types of single-well or multiple-well methods can be applied in this approach. The type of tracer test to use depends on the scale of the test and intended accuracy. Fried (1975) describes the different types of field tracer tests and their range of applicability. The second technique of evaluating dispersion coefficients is known as the inverse method. In the inverse method, dispersion coefficients are determined by simulatıng the time history migration of pollutant in an aquifer. This is accomplished by. varying the parameters in the dispersion model urttil the calculated concentration sontours match the historical data. A major limitation of the inverse method is that because of the large number of parameters used in transport modeling a unique solution is often not obtainable. Anderson (1979) presents a detailed discussion on the application and limitations of the inverse method.

Dispersion in anisotropic porous media is not well understood. No experimental or analytical technique is avaitable for evaluating all nine components of the classical dispersion tensor for an anisotropic medium (Anderson, 1979; Freeze and Cherry, 1979). Consequently, dispersion in anisotropic porous media cannot be modeled accurately. The current practice is to treat an anisotropic medium as an equivalent isotropic porous medium in dispersion studies. This simplification is made because the two independent coefficients of the disperson tensor for an isotropic porous medium can be evaluated. These two independent coefficients are the dispersion coefficient in the direction of flow called the longitudinal dispersion coefficient, and the dispersion coefficient perpendicular to the direction of flow called the transverse dispersion coefficient. All references sited in this literature review make the assumption that dispersion takes place in an isotropic porous medium.

One of the main reasons dispersion in anisotropic porous media is indeterminable is that the proper flow field to use in a tracer expcriment has not been recognized. The direction of flow coincides with the direction of the hydraulic gradient for an isotropic rorous medium; 
hence, it is easy to create a uniform flu. $y$ ficld as discussed earlier. However, for an anisotropic porous medium, the direction of flow and the direction of the hydraulic gradient do not coincide. Thus, when the hydraulic boundary conditions used in a tracer experiment for an isotropic medium are applied to an anisotropic medium, a nonuniform flow field is created. The appropriate flow field to use in tracer experiments to evaluate mechanical transport for anisotropic porous media will be introduced in Chapter 3.

There has been no justification to qualify the treatment of an anisotropic medium as an equivalent isotropic medium. Evidence from field experiments suggests that this simplification may lead to serious errors in transport prediction. The dispersion coefficients calculated from field tests are found to be many times larger than dispersion coefficients measured in laboratory experiments (Anderson, 1979; Fried,1981; Cherry et al.,1978). The difference ietween the two measured coefficients could be attributed to directional transport properties of the medium.

Another important finding is that the classical dispersion coefficients computed for field data commonly increase with time (Pickens and Grisak, 1981). The transient behavior of dispersion coefficients are commonly explained based on the scale of testing. Mechanical dispersion, commonly the controlling mode of dispersion, is caused by zones of velocity variations which cause particles to move at different rates and spread out spatially. The size of these zones of velocity variations is referred to as the scale of heterogeneity. As the scale of heterogeneity increases, the initial period of non-Fickian dispersion increases. Laboratory samples are farrly homogeneous and dispersion is caused by velocity variations within the microscopic pores. Field tests are conducted on a very iarge scale, so that dispersion results from macroscopic heterogeneous zones which are much larger than a pore. The large scale of heterozeneity influencing a field test results in measurements of transient dispersion coefficients which indicate tt at the perior of testing is within the initial period of non-Fickian dispersion.

In summary, Fickian dispersion for anisotropic porous media cannot be accurately modeled because the second-order dispersion tensor cannot be evaluated. When dispersion 
coefficients are computed by treating the medium as an equivalent isotropic medium, the calculated coefficients often increase with sample size. Both of these problems lead to difficulties in transport prediction. Methodology will be developed in Chapter 3 to evaluate both the hydraulic effective porosity and longitudinal mechanical transport coefficient for anisotropic porous media. The applicability of the Fickian approach to characterize mechanical transport for anisotropic fracture 3 rock masses will be investigated in Chapters 5 and 6 .

\subsubsection{Stochastic Models for Porous Media Dispersion}

The continuum approach is a black-box technique which computes the output from the box without analyzing what actually happens within the box. Stochastic modeling is a more detailed approach which analyzes the internal structure and actions within the box to determine the system's output. Analytical and numerical stochastic models are briefly reviewed to pro:ide the concepts necessary in developing a stochastic model. A numerical stochastic model that simulates mechanical transport in fractured rock is developed in Chapter 4. Since stochastic modeling is a fairly recent approach one of the objectives of reviewing these studies is to show the approaches being used in research on porous media transport.

\subsubsection{Analytical Stochastic Models of Dispersion}

In analytic stochastic models, certain transport parameters, such as the mean rate of advection, are allowed to vary statistically throughout the porous medium. This results in a stochastic dispersion equation which is then solved to determine macroscopic dispersion within the medium. Gelhar et al. (1979) investigated dispersion in vertically-stratified media. The focus of their study was to determine whether macroscopic dispersion in the aquifer could be represented by the classical approach. Horizontal flow was produced in each layer by introducing a uniform hydraulic gradient in the direction of flow. Dispersion was primarily caused by the variation of permeability in the vertical direction. Each variable in the classical dispersion equation, except effective porosity, was expressed as a stationary random variablc represented by its expected value plus a perturbation component. The stochastic equation for the concen- 
Iration perturbation was then derived neglecting all se sond-order perturbation terms. Spectral analysis was appied to solve this equation.

The rtsults of their study, for a specific permeability spectrum, indicated that global dispersion was Fickian only after a period of non-Fickian dispersion. Initjally, the variance of the plume increased much more rapidly than predicted by Fickian dispersion and the spatial distribution of the tracer cloud was highly skewed. The spatial distribution of the tracer cloud is symmetric when the Fickian approach is applicable. As time went on, Fickian transport was approached as the tracer cloud became more symmetric and the rate of spreading decreased.

Matheron and de Marsily (1981) analyzed dispersion in vertically stratified aquifers in which a vertical component of velocity existed. The following parameters in the dispersion equation were considered to be constants: vertical component of velocity, longitudinal dispersion coefficient, transverse dispersion coefficient, and effective porosity. This meant that the only stationary random variable in the dispersion equation was the horizontal component of velocity. The stochastic model traced the moviment of a solute particle through the aquifer. This tcchnique was equivalent to directly solving the dispersion equation because the probability density of the solute particle at a point was equal to the expectation of concentration at that point.

The spatial variance in the horizontal direction was found to be a function of the covariance in the horizontal velocity component. Fickian dispersion only occurred if this covariance satisfied ceriain conditions, and only after an initial period of non-Fickian dispersion. The vertical component of velocity caused the conditions for Fickian dispersion to be less stringent than if flow was strictly horizontal in the aquifer. Fickian dispersion occured early when the vertical velocity component and vertical dispersion coefficient were large. In a practical example involving a sandstone aquifer. Fickian dispersion was approached at app:oximately 140 days, or after 600 meters of travel under natural flow conditions. This amount of time is often not feasible for a field tracer experiment.

A stochastic study of macrodispersion in three-dimensional heterogeneous porous media 
was conducted by Dieulin et al. (1981). Macrodispersion is dispersion caused strictly by velocity variations within the heterogeneous porous medium. The velocity field was assumed to be a stationary random process in the Dieulin et al. study. The stochastic model traced the movement of a solute particle instead of directly solving the dispersion equation, the same technique used in the Matheron and de Marsily (1981) study. Dieulin et al. showed that the dispersion tensor can vary in magnitude with time, and afte- a sufficient initial period, the dispersion tensor may become constant.

\subsubsection{Numerical Stochastic Models of Dispersion}

Numerical stochastic dispersion modeling is a discrete approach to investigate dispersion in porous media. A porous medium is stochastically created within a problem domain by dividing the domain into a large number of small subregions and statistically senerating the dispersion parameters for each subregion. Next, hydraulic boundary conditions are applied to the domain to create the desired flow field. Reference tracer particles are then introduced into the porous medium and the detailed particle movements are monitored to evaluate dispersion.

One of the first stochastic studies in porous media transport was conducted by Warren and Skiba (1964). This work focused on interpreting the effect of the scale of heterogeneity on Fickian mechanical transport. A cubic porous medium domain was subdivided into constantm sized blocks that were cubic or rectangular parallelepiped. Block sizes varied with each Monte Carlo run to examine the influence of the scale of heterogeneity on dispersion. Permeaoility was lognormally distributed withm the blocks, and porosity was normally distributed within the blosks.

Boundary conditions were applied to the domain to induce flow in one direction between two opposing sides and reference particles were randomly introduced across the domain's inlct. The magnitude and direction a particle was displaced in a discrete time interval was determined by interpolating velocities in the blocks adjacent to that containing the par-icle. As particles exited the system, the travel time of each particle was recorded from which the breakthrough curve was constructed. The classical longitudinal dispersion coefficient was then 
computed from this breaithrough curve.

Warren and Skiba found that the longitudinal dispersion coefficient increaseo as the ratio of the length of a block to the width of the domain decreased. This implies that the longitudinal dispersion coefficient is unque (Fickian dispersion) for all sample sizes only if this dimensionless length ratio remains constant. This also implies that for Fickian dispersion, the scale of heterogeneity must increase with sample size.

Schwartz (1977) investigated the vaiidity of the Fickian approach for characterizing mechanicai transport in heterogeneous porous media. In his study, two-dimensional rectangular porous media domains were constructed using equal-sized rectangular elements. Heterogeneity was created by distributing low-permeability elements within a background of highperm zability elements. Three types of porous media were simulated by distributing the lowpermeability elements in different patterns: random, regular, and aggregated, in which the lowpermeability elements appeared as clusters within the domain. A one-dimensional flow field was established, and reference particles vere introduced across the inlet to tne domain. Schwartz investigated the apr,'icability of the classical approach by computing the spatial variance of the particle distribution with time. The spatial variance increases linearly with time when the longitudinal dispersion coefficient is unique.

Schwartz found that the applicability of the classical approach was dependent on the type of porous medium. In a stratified medium, created by generacing three layers of low permedbility elerr ents within the domain, Schwartz found that a unique dispersion coefficient did not exist. A unique dispersion coefficient was approached as the number of elements within the domain increased and as the distribution pattern for the low-permeability elements became random. The longitudinal dispersion coefficient increased with increased permeability contrast and with increased randomness of the low permeability elements.

In general, the Fickian longitudinal dispersion coefficient computed by Sch:vartz (1977) overestimated the spatial variance of the particle distribution in early stages of dispersion, and underestimated it in later stages for a given realization. The particles moved with about the 
same velocity in the early stages of dispersion so the rate of dispersion was small. However, with time, the heterogeneities caused the particles to move at different rates and, consequenty, the spatial variance increased rapidly.

Smith and Schwartz (1980,1981a,b) extended the earlier work of Schwartz (1977). A two-dimensional porous medium domain was created using a first-order nearest-neighbor stochastic model in which the permeabilities of neighboring elements were spatially correlated. The details are given in Smith and Schwartz (1980). A hybrid deterministic-probabilistic method governed the movement of tracer particles. The deterministic stage was similar in concept to Warren and Skiba (1964). The probabilistic stage accounted for the effects of microscopic dispersion such as molecular diffusion.

Two tests were performed to evaluate if macroscopic dispersion could be represented by a unique longitudinal dispersinn coefficient. The first test, identical to Schwartz (1977), investigated the linear variation in the spatial variance with time. A stronger condition for Fickian dispersion is that the spatial particle distribution should be Gaussian. To test for this requirement, the chi-square test was used.

In a Monte Carlo simulation totaling 300 realizations, Smith and Schwartz found that less than 18 percent of the realizations satisfied the two criteria for Fickian dispersion. Smith and Schwarz stated the condition necessary for Fickian dispersion:

"Fundamental to the diffusional representation of dispersion (Scheidegger, 1964) is the assumption that as the total particle iravel time becomes much greater than the time interval during which its successive local velocities are still correlated, then the total displacement may be considered as the sum of a large number of elementary displacements statistically independent of one another."

The total particle travel times were too short in the Monte Carlo simulation for the Fickian representation of dispersion to be applicable. Multimoda! particle distributions found in many realizations clearly showed that successive local velocities were correlated. Particles traveled along preferred pathways and thus, were sampling only a portion of the velocity field. Consequently, connected pathways of rapid movement/high permeability and slow movemen:/low permeability developed withir the domain. 
Stochastic dispersion studies have demonstrated that global dispersion in heterogencous porous media is characterized by an initial period of non-Fickian dispersion. The spatial variance of the solute plume spreads very rapidly in this initial period such that an equivalent classical dispersion coefficient would have to increase with time in order to characterize dispersion. These conclusions agree with observations made from field tests. After this initial period, Fickian dispersion may occur depending on the statistical properties of the velocity field. Fickian dispersion is likely to occur in a porous medium with a small scale of heterogeneity and a random velocity fieid.

\subsection{DISCRETE MECHANICAL TRANSPORT MODELS FOR FRACTURED ROCK MASSES}

Three discrete mechanical transport models for fractured rock masses are reviewed in this section. Detailed discussions are presented on the approaches used to simulate mechanical transport in each model. A new discrete mechanical transport model will be developed in Chapter 4 that is based on fundamental principies of fluid flow.

Neretnieks (1980) analyzed mechanical transport in a set of parallel, planar, and continuous fractures. A one-dimensional flow field was established, parallel to the orientation of the set, by a uniform hydraulic gradient. Fracture apertures were aistributed according to a known probability distribution. The velocity in ach fracture was assumed equal to the average crosssectional velocity which was proportional to the cubed power of the aperture. Thus, mechanical transport was caused by the variation in fracture velocities produced by the aperture distribution. The composite concentration distribution at the exit end of the problem domain was analytically solved for both a step and a puise injection of concentration across the inlet to the domain. Neretnieks found that Fickian macroscopic transport did not occur for this fracture system because the longitudinal dispersion coefficient increased with sample size. This result was not surprising since this fracture system was equivalent to a stratified porous medium. The work of Neretnieks considered fractures whish had no interactions with the other fractures 
in the system. The following two studies dealt with more realistic networks of intersecting fractures.

In a series of four related papers (Castillo et al., 1972; Krizek et al., 1972; Karadi et al., 1972; and Krizek et al., 1973), a numerical model was developed to simulate transport in a fracture system consisting of two sets of parallel, slanar, and continuous fractures of constant aperture and spacing. The principles incorporated in their model can be understood by following the migration of solute as it flows through the fracture system. Mechanical transport governs the movement of solute within an element:

$$
\frac{\partial c}{\partial t}+u(\eta) \frac{\partial c}{\partial \xi}=0
$$

When solute exiis an element at a node, it encounters solute from other elements flowing into the node. Krizek et al. used either the complete mixing condition or the partial mixing condition to distribute the inflowing solute to the outflow elements. In the complete mixing cundition, all outflow elements receive the same concentration given by :

$$
c_{0}(t)=\frac{\sum_{i} \int_{0}^{h_{1}} u_{i}(\eta) c_{i}(\eta, t) d \eta}{Q_{N}}
$$

where the summation of $i$ is for all inflow elements. The complete mixing condition is illustrated in Figure 2-2a.

The partial mixing condition is illustrated in Figure 2-2b. Fluid enters the node from elements $A$ and $B$, and exits through elements $C$ and $D$. Element $D$ is a large element such that the flow rate in element $D$ consists of the total flow rate in element $A$ and part of the flow rate in element $B$. The widths in elements A and B occupied by the flux flowing into element $D$ are determined, and the mass of solute entering the node from thes: zones is calculated. This mass of solute is then distributed uniformly across the entrance to element $D$.

The travel time within a node is usually very shor, such that the solute is primarily advected by the flow in a node. The physics of laminar flow suggest that the two mixing conditions above would not properly simulate transport within a node. In laminar flow, the fluid movos in layers. one layer flowing smoothly over the adjacent layers. A flow lamina cannot 
Arrow indicates direction of flow
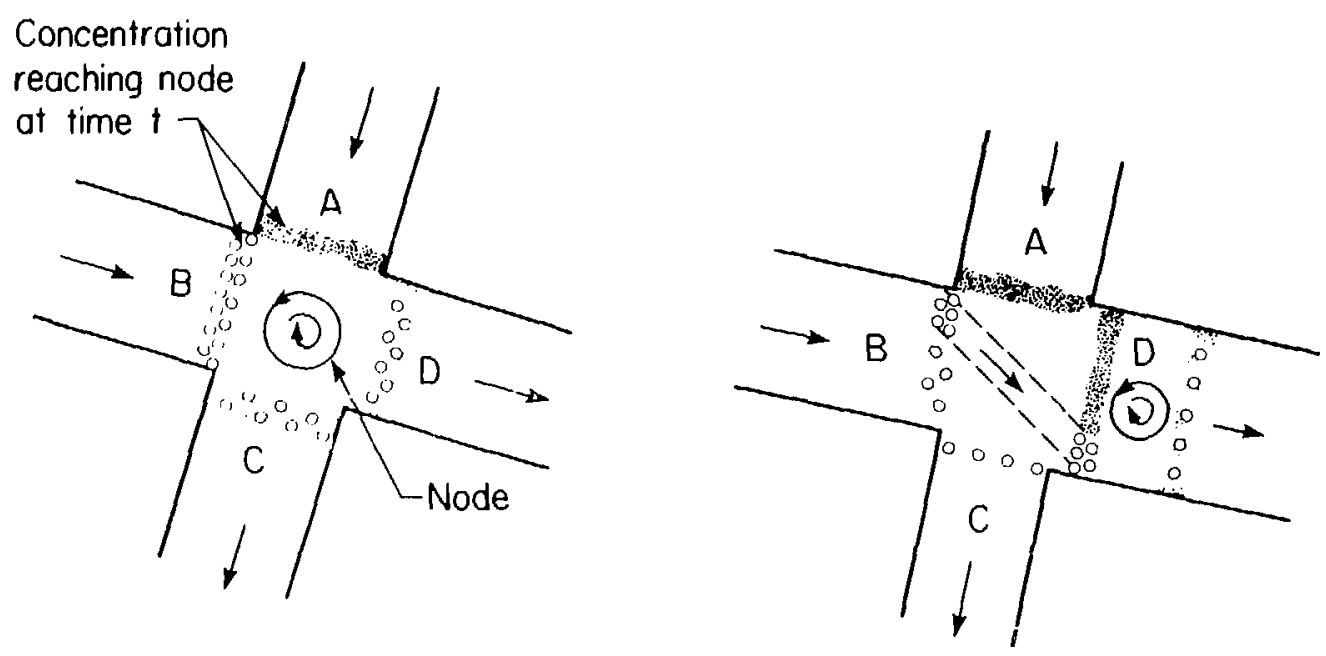

(a) Complete mixing condition

(b) Partial mixing condition

Figure 2-2 Graphical Representation of Mixing Conditions at a Node.

XBL $833-8772$ 
cross over into another layer, as assumed in the above model, to develop uniform concentrations across the entrance of outflow elements. Although laboratory experiments performed by Krizek et al. (1972) concluded that the complete mixing is valid for laminar flow, their experiments were not conclusive. Tiese experiments were limited to the situation in which there was only one inflow element and all outflow elements had nearly identical flow rates. A more general set of experiments should be considered which involves more than one inflow element, with unequal flow rates and unequal concentrations in both inflow and outflow elements. Wilson (19\%0) conducted such an experiment using capillary tubes for Reynolos number on the same order as the experiments by Krizek et. al (1972) and demonstrated that fluid still flows within layers at intersections when the flow is laminar.

Schwartz et al. (1981) stochastically generated two orthogonal fracture sets in a rectangular domain. The number of fractures in each set within the domain was controlled by the areal density (the number of fractures per unit area). The fracture centers were randomly distributed in the domain and the fracture lengths and apertures were generated from exponential and lognormal distributions, respectively. Schwartz et al. avoided a major simplification made in previous transport studies of fracture systems by using fractures of finite length. Impermeable boundaries were created on two opposite sides of the rectangular domain and the two remaining sides consisted of constant-head boundaries. Tracer particles were assumed to propagate at a constant velocity within the elements and were also assumed to be completely mixed at the nodes, even though the flow was laminar. Moleculat diffusion was considered to be negligible within a fracture and therefore, was not modeled.

Schwartz et al. (1981) found that the spatial particle distribution in the average direction of flow was non-Gaussian and positively skewed. The skewness is attributed to a combination of channels of rapid movement oriented in the direction of flow, and channcls of slow movement oriented normal to the direction of flow. The bulk of the flund moves in the direction of gradient, and most of the particles travel in this direction. However, as time proceeds, the probability of a particle flowi.ag into a slow channel increases. As particles move through the slow 
channels, an asymmetrical particle distribution develops.

Consequently, previous mechanical transport models simulate transport in twodimensional fracture networks by linking together one-dimensional or quasi-o:je-dimensional mechanical transport models for each element in the network as shown in Figure 2-3. The model is one-dimensional because a constant velocity is assumed to exist in the element or a nodal mixing condition which distributes concentration uniformly across the entrance of outflowing elements is used. Completely or partially neglecting the effects of the velocity distribution across a fracture ignores an important microscopic mechanism of mechanical transport. The nodal mixing conditions used in these models are not based on the physics of laminar flow. In laminar flow, the $\mathrm{It}$ id moves in layers, one layer flowing smoothly over the adjacent layers. A flow lamina cannot cross over another layer, as assumed in these models, by uniformly distributing concentration across the entrance of the outflow elements at a node. A two-dimensional mechanical transport model for each element is developed in Chapter 4 to provide a more realistic understanding of mechanical transport. 


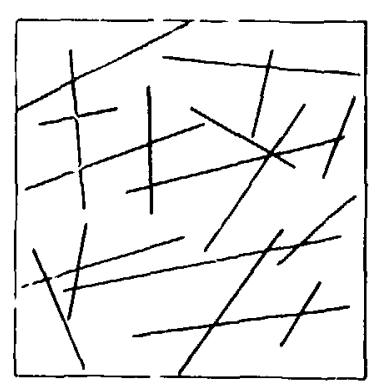

Two dimensional

fracture system

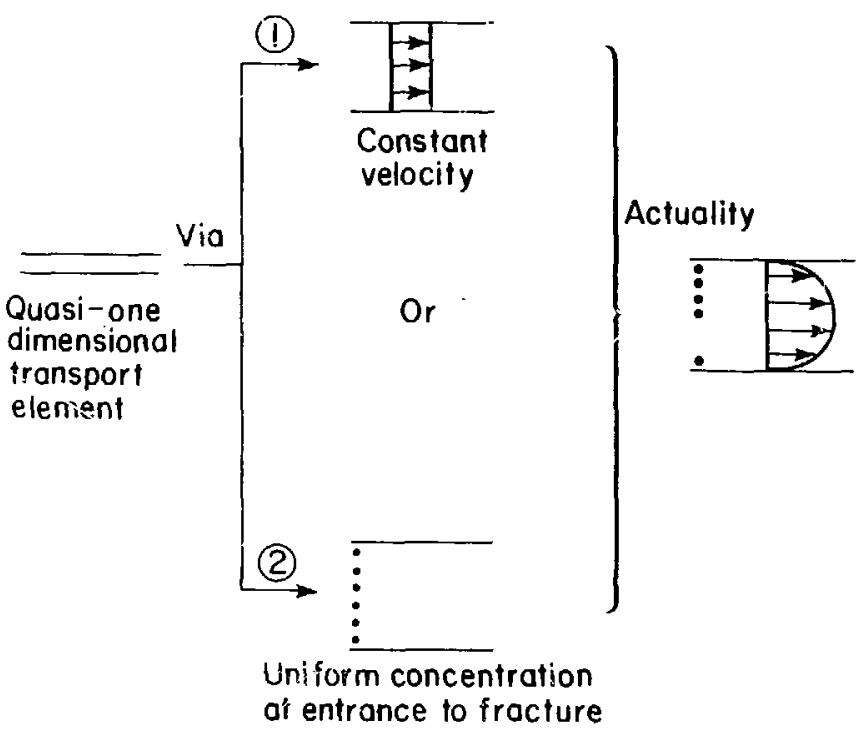

XBL-829-4506 


\section{CHAPTER 3 \\ THEORETICAL DEVELOPMENT}

\subsection{INTRODUCTION}

Transport is consi Jered at the microscopic, pore, and continuum levels in the first part of this chapter. Transpori is modeled within a very small differential control volume at the microscopic level. Consequently, all transport prucesses are accurately modeled at this level. In porous media modeling, it is extremely difficult to use a microscopic transport model because oi inc complex flow pattern within the fores and the irregular structure of the pores. The only practical way of modeling transport in a porous medium is to treat the medium as a continuum and analyze the macroscopic behavior of the medium, disregarding the detailed beharior occurring within the pores. Both rock and fluid properties var; smoothly and continususly throughout the continuum as there is no physical boundary between solid and fluid phases. However, the concepts oi continuum modeling can lead to difficulties in accurately modeling transpônt processes. Transport models are derived at the microscnpic, pore, and continuum levels :o demonstrate the assumptions and simplifications that are needed to model transport at each level.

No experimental technique is avajlable which can evaluate dispersion in anisotropic porous media, and consequently, an anisotropic medium is treated as an equivalent isotropic medium (Anderson, 1979). This simplification assumes that there is no directional dependence in each transport parameter. A set of tracer experiments will be presented in this chapter that allow the directional char acteristics of mechanica! transport for anisotropic media be evaluated. The design, execution, and cetermination of mechanical transport parameters from the set of experiments are presented in this chapter.

A discrete model is developed in Chapter 4 to simulate fluid flow and mechanical iransport in fractured rock masses. An important objective of this research is to determine if a fracture system can be treated as an equivalent porous medium continuum. The conditions 
required for equivalent porous medium beiavior are discussed below. Tests are developed, based on these conditions, to detect when a fracture system can be treated like an eucuivalent porous medium. These tests are incorporated into the numerical model developed in Chapter 4.

\subsection{MICROSCOPIC LEVEL TRANSPORT}

To develop a transport model at the microscopic level, a conservative solute with the same viscosity and density as the ambient fluid is migrating in a porous medium. The model is derived below, taking into account the cubic differential control volume shown in Figure 3-1. The two microscopic transport processes occurring are molecular diffusion and advection. Molecular diffusion is the mixing caused by the randiom motion of solute molecules produced by the concentration gradient. Advective transfort is caused by the movement of fuid within the pores. The modeling of each transport process at the microscopic level is presented below.

The diffusive mass flux is defined as the mass of solute diffusing across a unit area in a unit time. The diffusive mass flux is governed by Fick's law which states:

$$
\overrightarrow{\mathbf{d}}=-\mathrm{D} \nabla \mathrm{c}
$$

The net mass flux diffused into the control volume in the $x$ direction in Figure 3-1 is equal to:

$$
\text { [mass flux diffused into face } 1]-[\text { mass flux diffused out of face } 2]
$$

which is equal to:

$$
\left[d_{x}-\left(d_{x}+\frac{\partial d_{x}}{\partial x} d x\right)\right] d y d z=\frac{\partial\left(D \frac{\partial c}{\partial x}\right)}{\partial x} d x d y d z
$$

Similarly, the net mass flux diffused into the control volume in the $y$ and $z$ directions are, respective!y;

$$
\frac{\partial\left(D \frac{\partial c}{\partial y}\right)}{\partial y} d y d x d z \text { and } \frac{\partial\left(D \frac{\partial c}{\partial z}\right)}{\partial z} d z d x d y
$$

The advective mass flux is defined as the mass of solute advecting across a unit area in a unit time. The total mass advected into the control volume per unit time across face 1 is equal to the advective mass flux at face $I\left(v_{2} c\right)$ multiplied by the area of face 1 : 

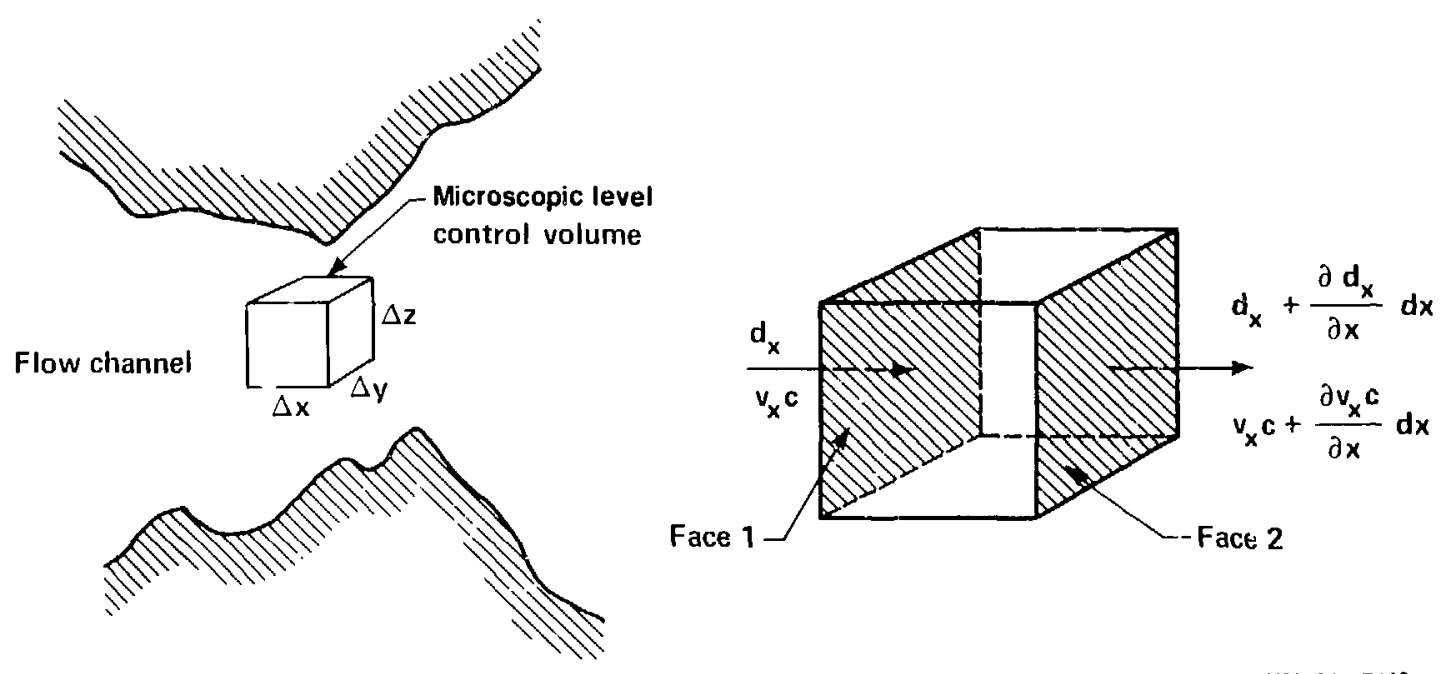

Figure 3-1 Modeling of Transport Within a Control Volume at the Microscopic Level. 


\section{$\left(v_{x} c\right) d y d z$}

Following a similar procedure for net advective transport as demonstrated for diffusive transport, the net mass flux advected into the control volume in the $x, y$, and $z$ directions are. respectively:

$$
-\frac{\partial\left(v_{x} c\right)}{\partial x} d x d y d z,-\frac{\partial\left(v_{y} c\right)}{\partial y} d x d y d z,-\frac{\partial\left(v_{z} c\right)}{\partial z} d x d y d z .
$$

The mass conservation of solute within the control volume states that:

$$
\left[\begin{array}{l}
\text { net mass flux of } \\
\text { solute diffused } \\
\text { into control volume }
\end{array}\right]+\left[\begin{array}{l}
\text { net mass flix } \\
\text { adverted into } \\
\text { control volume }
\end{array}\right]=\left[\begin{array}{l}
\text { net accumulation of } \\
\text { solute in control } \\
\text { volume per unit time }
\end{array}\right]
$$

Substituting the appropriate terms into the equation above and simplifying yields:

$$
D \nabla^{2} c-\left(\frac{\partial v_{x} c}{\partial x}+\frac{\partial v_{y} c}{\partial y}+\frac{\partial v_{z} c}{\partial z}\right)=\frac{\partial c}{\partial t}
$$

which is the partial differential equation governing transport a' the microscopic level. The first term on the left hand side of equation 3.1 is called the dispersive term and the bracketed term is called the advective term. Equation 3.1 is extremely difficult to solve for a porous medium because of the complicated flow pattern within the pores and the complex boundary conditions arising from the random geometry of the pores. Therefore, the continuum approach is almost exclusively used to model porous media transport. The analysis of the microscopic flow field within the pores is avoided in the continuum approach because macroscopic properties of the medium are evaluated. Also, there is no neeu to define the detailed geornetry of the pores because both rock and fluid properties vary continuously throughout the continuum, since there is no physica! boundary beiween fuid and solid phases. Before the transport model for porous media is developed, transport models are discussed for smailer scale transport within a single flow conduit (i.e. pipe or a single conductive pore within a porous medium). These pore transport models must simulate the velocity field within a pore.

\subsection{PORE TRANSPCIRT MODELS}

The first pore trarsport model presented is the dispersed plug flow model. The flow velocity in equation 3.1 is r:splaced by iue average cross-sectional velocity in the dispersed plug flow 
model. This means that in this model solute is advected at the same rate across the cross section of a flow channel. Consequently, the dispersive term in the dispersed plug flow model must account for both molecular diffusion and mechanical dispersion praduced by the crosssectional velocity distribution. Since dispersion is greater in the longitudinal direction than in the transverse direction, because of mechanical dispersion, the constant coefficient, $D$, in equation 3.1 is replaced by a second-order dispersion tensor $\left[D_{F}\right]$ to account for the anisotropic rate of dispersion. Thus, the apparent simplification made to the advective term is somewhat negated by the added complexity introduced into the dispersive term.

Consider, for example, transport in a parallel plate conduit with the channel axis corresponding to the $\mathrm{x}$ direction as shown in Figure 3-2. The dispersed plug flow model for the parallel plate conduit is:

$$
\frac{\partial c}{\partial t}+\vec{v} \frac{\partial c}{\partial x}=v\left(\left|D_{F}\right| \nabla c\right)
$$

The second pore transport model presented is the axial dispeised plus fow model. The axial dispersed plug flow model makes an additional simplification to the dispersed plug flow model by assuming that concentration is uniformly distributed across each section such that no concentration gradient exists in the transverse direction. Consequently, dispersion occurs only in the longitudinal direction and the dispersive coefficient, which is multiplied by $\nabla^{2} \mathrm{c}$ in the dispersive term, is a scalar, $D_{L}$. The axial dispersed plug flow model for transport in a parallel plate conduit is:

$$
\frac{\partial c}{\partial \mathrm{t}}+\bar{v} \frac{\partial c}{\partial \mathrm{x}}=\mathrm{D}_{\mathrm{L}} \frac{\partial^{2} \mathrm{c}}{\partial \mathrm{x}^{2}}
$$

The axial dispersed plug flow model cannot simulate transport during the initial period of transport. For axample, consider the laminar flow problem of transpor in a paral!el plate conduit when a pulse of solute is instantaneously injected uniformly acruss the entrance to the conduit. as shown in Figure 3-2. The axial dispersed plug flow model is applicable when there is no concentration gradient in the transverse direction. In the early stages of transpor, the solute distribution wilt violate this condition because advective transport is dominant. as shown in Figure 3-2. However, as time increases, molecular diffusion slowly reduces the concentration 


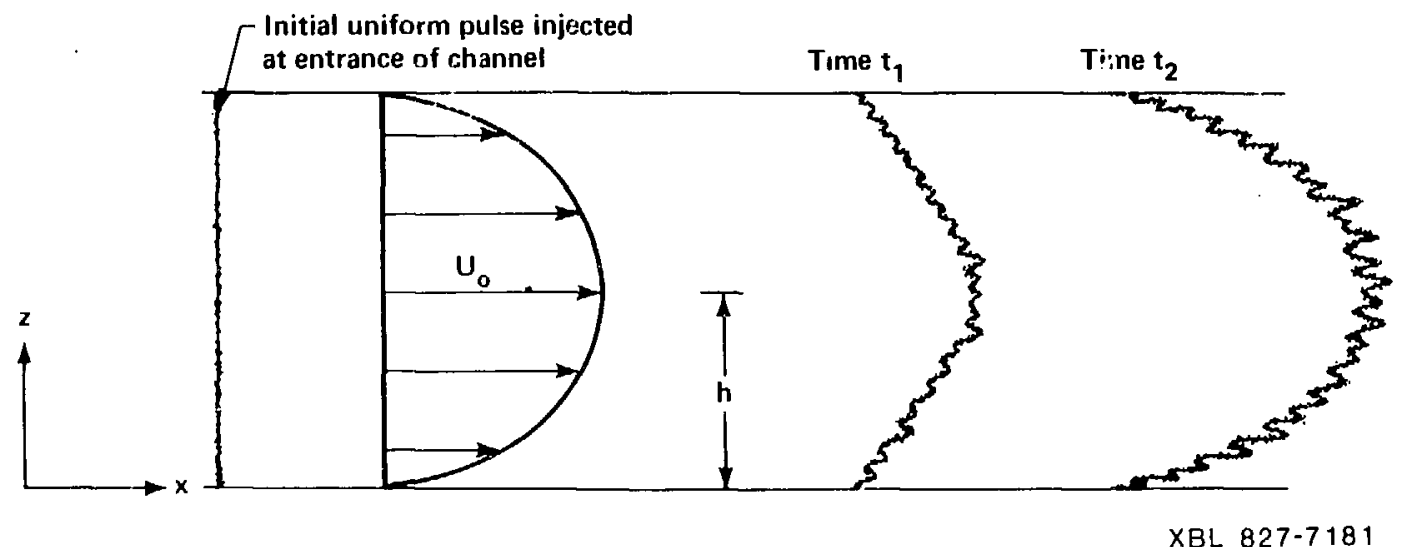

Figure 3-2 Early Stages of Transport When a Uniform: Pulse is Injected Between Two Parallel Plates Under Laminar Flow Conditions. 
gradient in the transverse direction. Gill et al. (1969) used a numerical technique to show that, for times greater than $h^{2} / D$, the axial dispersed plug flow is applicable.

Gill et al. (1969) demonstrated that the axial dispersed plus flow model cannot simulate early-time transport. The averaging of the flow velocity and avoidance of transvarse dispersion indicate that a start-up time is required before the axial dispersed plug flow model is applicable. This initial pericd is similar to the period of non-Fickian dispersion commonly observed in porous media field tests. The cause of this period of non-Fickian dispersion in porous media is related to the macroscopic averaging of properties in the transport model for porous media. The derivation of the porous medium transport model is presented in the next section.

\subsection{POROUS MEDIA TRANSPORT MODEL}

The transport model for porous media is derived by considering mass conservation within a cubic continuum control volume much larger than a pore consisting of solid and fluid phases. The dimensions of this continuum and the coordinate system used in this derivation will be identical to those shown in Figure 3-1. In a continuum, fluid and solid phases in:st at every point within the medium such that both rock and fluid properties are smooth and continuous functions througbout the medium. The porous media transport model consists of an advective transport process coupled with a dispersive transport process The advective transport process will be modeled first.

The mass of solute advected into the control volume per unit time across face 1 is $Q_{x} c$. The flow rate $Q_{x}$ is usually expressed in terms of the specific discharge. The specific discharge is defined as the flow rate crossing a unit area of porous medium continuum and is governed by Darcy's law which states:

$$
\Phi=\frac{\rho g}{\mu}|\mathbf{k}| \vec{\jmath}=|\mathrm{K}| \vec{\jmath}
$$

The specific discharge is often nisconstrued as representing a ijpe of velocity, because the dimensions of $\vec{q}$ are the same as the dimensions for velocity. However, the specific discharge is the quantity of flu'd flowing across a unit area per unit time, and should never be confused with velocity. The flow rate crossing a given area of gorous medium uritten in terms of the 
specific uischarge is:

$$
Q=\vec{q} \cdot \vec{A}=\mid K \sqrt{j} \cdot \vec{A}
$$

Consequenty, the mass of solute advected into the contro! volume across face 1 per unit time can be expressed as:

$$
\left(g_{x} d y d z\right) c
$$

It is apparent from equation 3.2 that the transport modei for porous media can be classified as a dispersed plug flow model because soiute is being advected across face 1 into the control volume at a constant rate of $q_{x} d y d z$. In reality, solute is advected into the control volume from the pores at different rates. The microscopic viriations in the advective rate of transport are accounted for in the dispersive term.

The net mass flux advected into the control volume in the $x$ direction is:

$$
\left[q_{x} c-\left(q_{x} c+\frac{\partial q_{x} c}{\partial x} d x\right)\right] d y d z=-\frac{\partial\left(q_{x} c\right)}{\partial x} d x d y d z
$$

Similarly, the net mass flux advected into the control volume in the $y$ and $z$ directions are, respectively:

$$
-\frac{\partial\left(q_{y} c\right)}{\partial y} d y d x d z \text { and }-\frac{\partial\left(q_{z} c\right)}{\partial z} d z d y d x
$$

Advective transport alone causes no distortion in the shape of a pollutant plume. The distortion and spreading of a plume is caused by microscopic variations in the advective rate of transpor, termed mechanical transpor, and by molecular diffusion. Thus, the dispersive term accounts for the combined interaction of mechanical dispersion and molecular diffusion known as hydrodynamic dispersion.

The principle microscopic mechanisms of mechanical transport, as discussed in section 2.2.2 and illustrated in Figure 2-1 are: the velocity distribution across a prote which cavses a fluid particle to move faster in the center than along the sides of the pore; the flow rate variation from one channel to another which dictates the direction a fluid paticle will travel; and the randon geometry of the pores whicl. aluses a particle to meandel through the pore region. The mechanizal dispersive flux is the mass of solute mechanically transported across a unit area 
in a unit time. The mass flux of solute mechanically dispersed into the control volume across face 1 is:

$$
\mathbf{m}_{\mathbf{x}}\left(\phi_{\mathrm{R}} \mathrm{dydz}\right)
$$

where $\phi_{R}$ is the rock effective porosity defined as the conductive void volume per volume of rock. The net mass flux mechanically dispersed into the control volume in the $\mathrm{x}$ direction is:

$$
-\frac{\partial \mathrm{m}_{\mathrm{x}}}{\partial \mathrm{x}} \phi_{\mathrm{R}} \mathrm{dxdydz}
$$

Similarly, the net mass flux mechanically dispersed into the control volume in the $y$ and $z$ directions are, respectively:

$$
-\frac{\partial m_{y}}{\partial y} \phi_{R} d x d y d z \text { and }-\frac{\partial m_{z}}{\partial z} \phi_{R} d x d y d z
$$

The Fickian expression developed by Scheidegger (1961) is commonly used to represent the mechanical dispersive flux:

$$
\mathbf{m}_{\mathrm{i}}=-\mathbf{M}_{\mathrm{ij}} \frac{\partial c}{\partial \mathbf{x}_{\mathbf{j}}}
$$

where

$$
M_{i j}=\alpha_{i j m n} \frac{v_{m} v_{n}}{V L I N}
$$

The fourth-order geometric dispersivity .ensor has a total of 81 components. Scheidegger showed that $[\alpha]$ possesses two symmetric properties, namely:

$$
\alpha_{\mathrm{ijmn}}=\alpha_{\mathrm{ijnm}}
$$

and

$$
\alpha_{\mathrm{ijmn}}=\alpha_{\mathrm{jimn}}
$$

so that there are 36 independent components in $|\alpha|$.

Presently, there is no experimental or numerical technique that will allow evaluation of all 36 independent components in $[\alpha]$ for an anisotropic porous medium. Consequently, an anisotropic porous rredium is generally treated as an equivalent isotropic medium because there are only two measurable components in $[\alpha \mid$ for an isotropic medium: 1) the longitudinal dispersivity $\alpha_{1}$, and 2) the transverse dispersivity $\alpha_{\mathrm{t}}$. The Fickian mechanical dispersion tenso- for an isotropic mecium with the direction of flow corresponding to the $\mathrm{x}$ direction is: 


$$
[\mathrm{M}]=\left[\begin{array}{ccc}
\alpha_{1} \mathrm{VLIN} & 0 & 0 \\
0 & \alpha_{\mathrm{t}} \mathrm{VLIN} & 0 \\
0 & 0 & \alpha_{\mathrm{t}} \mathrm{VLIN}
\end{array}\right]
$$

The mass flux diffused into the control volume is governed by Fick's law. Fluid flows along nonlinear paths in a porous medium due tc the irregular geometry of the pores as shown in Figure 3-3 for a single pore. However, the concentration gradient is considered in the linear directions aligned in the directions of the cartesian coordinate system $(x, y, z)$. To account for the nonlinear path of fluid movement in the diffusive mass flux, the free solution molecular diffusion coefficient, D, is divided by the tortuosity (Gilham and Cherry, 1982). The tortuosity is defined as the ratio of the mean path length of fit id flow to the linear length of travel. Thus the diffusive mass flux is:

$$
\bar{d}=-\frac{D}{\tau} \nabla c=-D_{0} \nabla c
$$

The total void volume in a porous medium consists of isolated zones, dead-end spaces, and conductive void regions. Mrlecular diffusion occurs within the dead-end and conductive void spaces. However, mechanical transport occurs only within the conductive void volume. The conductive void volume and total void volume are assumed to be equal in porous media transport modeling because of the difficulty of accurately differentiating the three void spaces. This assumption is generally valid because porous media are commonly highly interconnected such that dead-end zones and isolated segments usually uccupy only a small portion of the total void volume. Thus, the net mass flux diffused into the control voluine in the $x, y$, and $z$ directions are, respectively:

$$
D_{0} \frac{\partial^{2} c}{\partial x^{2}} \phi_{R} d x d y d z, \quad D_{0} \frac{\partial^{2} c}{\partial y^{2}} \Phi_{R} d x d y d z, \quad D_{0} \frac{\partial^{2} c}{\partial z^{2}} \phi_{R} d x d y d z
$$

The net increase in the mass of solute within the control volume per unit time is:

$$
\frac{\partial c}{\partial t} \phi_{R} d x d y d z
$$

The mass balance in the control volume states that:

$$
\left[\begin{array}{l}
\text { iat mass flux of } \\
\text { solute dispersed } \\
\text { into control volume }
\end{array}\right]+\left[\begin{array}{l}
\text { net mass flux } \\
\text { advected into } \\
\text { conirol volume }
\end{array}\right]=\left[\begin{array}{l}
\text { net accumulation of } \\
\text { solute in control } \\
\text { volume per unit time }
\end{array}\right]
$$




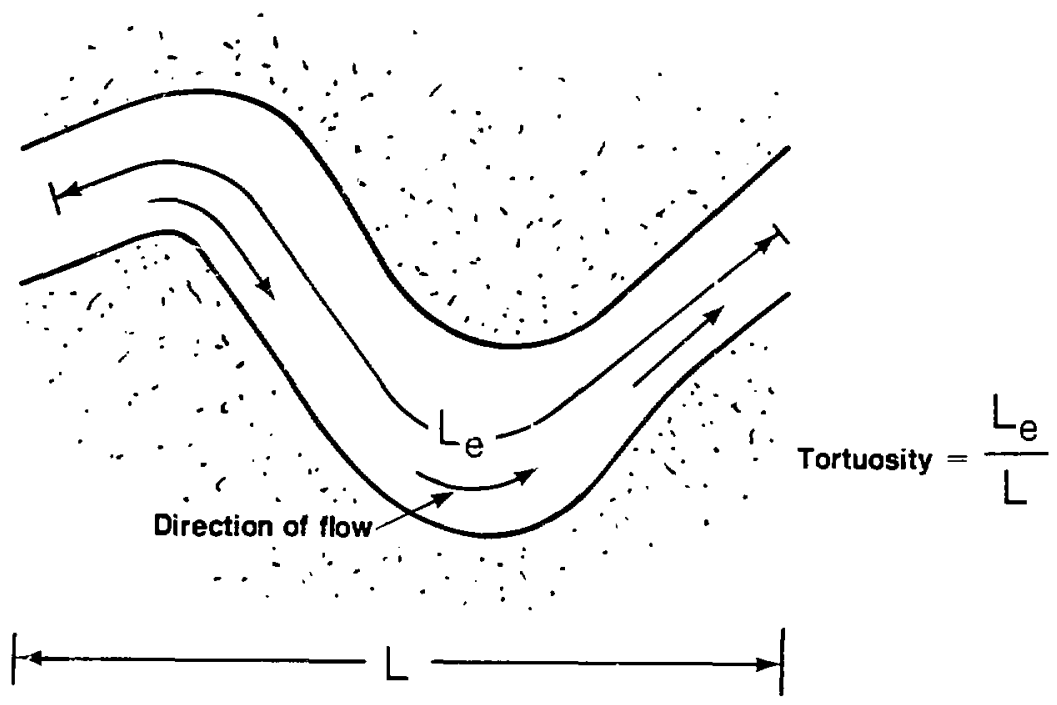

XBL 838-554

Figure 3-3 Tortuosity for a Single Pore. 
which, when substituted by the appropriate terms yields:

$$
\frac{\partial c}{\partial t}+\frac{1}{\phi_{R}}\left(\frac{\partial q_{x} c}{\partial x}+\frac{\partial q_{y} c}{\partial y}+\frac{\partial q_{z} c}{\partial z}\right)=D_{0} \nabla^{2} c-\nabla \cdot \vec{m}
$$

Equation 3.5 represents molerular diffusion and mechanical transport. The two terms on the right hand side of equation 3.5 constitute the dispersive term, while the bracketed term on the left hand side is the advective transport component.

This particular research is concerned with pure meshanical transport. The governing mechanical transport equation is:

$$
\frac{\partial \mathrm{c}}{\partial \mathrm{t}}+\nabla \cdot \overrightarrow{\mathrm{m}}+\frac{1}{\phi_{\mathrm{R}}}\left(\frac{\partial \mathrm{q}_{\mathrm{x}} \mathrm{c}}{\partial \mathrm{x}}+\frac{\partial \mathrm{q}_{\mathrm{y}} \mathrm{c}}{\partial \mathrm{y}}+\frac{\partial \mathrm{q}_{\mathrm{x}} \mathrm{c}}{\partial \mathrm{z}}\right)=0
$$

The three primary reasons for investigating mechanical transport are: (1) an insufficient understanding of hydrodynamic dispersion because no solution is available for the equatior governing transport in an anisutropic medium, (2) the use of mechanical transport parameters, such as tortuosity, in modeling hydrodynamic dispersion. and (3) the need to determine when fracture systems can be treated like equivalent porous media. New f.inciples and techniques will be introduced such that directional mechanical transport parameters can be evaluated for anisotropic media. The directional characteristics of hydraulic effective porosity will be used to develop the conditions under which fracture systems exhibit porous media equivalence. Consequently, the investigation of the fundamental mode of mechanical transport will lead to a beiter understanding of dispersion in all permeable media.

A series of tracer experiments must be conducted to evaluate directional mechanical $t_{1}$ nnsport for anisotropic porous media. The operation of the set of tracer experiments is discussed in two parts. First, two key steps in designing :he tracer experiment are discussed: the proper flow field to use in a tracer experiment, and the appropriate test section in measure mechanizal transport from a tracer experiment. Then. the execution of the set of tracer experizients is discussed. 


\subsection{PROPER FLOW FIELD AND APPROPRIATE TEST SECTION USED IN A TRACER EXPERIMEN1}

Two key steps in designing a tracer experiment to measure mechanical transport are: 1) selecting the proper flow field to use in the experiment and; 2) determining the appropriate test section within tue sample in which to measure mechanical transport. The macroscopic flow characteristics within the flow region must be consistent in order for general mechanical transport properties to be measured from a tracer experiment. The flow characterisuics are consistent when the flow field is uniform such that the specific discharge is constant throughout the flow region. If $\vec{q}$ varies from point to point in the medium, equation 3.6 becomes nonlinear and difficult to solve.

To establish this desired flow system, certain hydraulic boundary conditions mus. be maintained on the flow region. Figure 3-4 illustrates the hydraulic boundary conditions that are designed to create a uniform now field for an anisotropic, homogeneous porous medium. First, as shown in Figure 3-4a, constant hydraulic heads of $\mathbf{H}$ and 0 , respectively, are fixed on sides 2 and 4 of the flow region. Then, constant hydraulic gradients are maintained along sides 1 and 3. A constani hydraulic gradient in the flow fisld is needed to guarantee that $\vec{q}$ is uniform 'hroughout the how region in accordance with Darcy's lav'.

Figure $3-4$ also illustrates how flow net theory can be used to describe the flow field in $d$ homogeneous, anisotropic porous medium. Figure 3-4b shows the flow net in the transformed isotropic space (Freeze and Cherry. 1979, pr . :74-178), and Figure 3-4c shows the uniform flow: field for the anisotropic medium. The direction of flow is parallel to the streamlines and the hydraulic gradient is nomal to the equipotentia lines.

The proper test section within the flow region to conduct measurements of mechanical transpor must be selected. The tracer experiment will monitor the detailed movement of fluir? that enters the flow region on side 2 in Figure 3-4a. and exits on side 4 . In the proper test section, the linear length of travel is constant for the fluid which flows continuously between sides 2 and a This rejuirement guarantees that mechanical transpon properties are measured from 


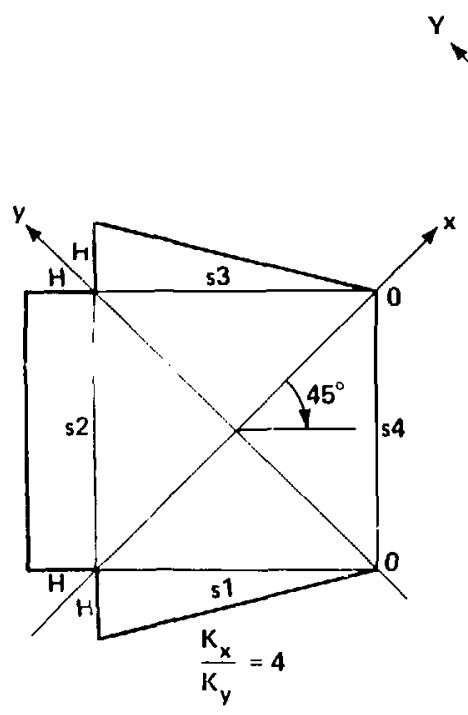

Flow Problem

(a)

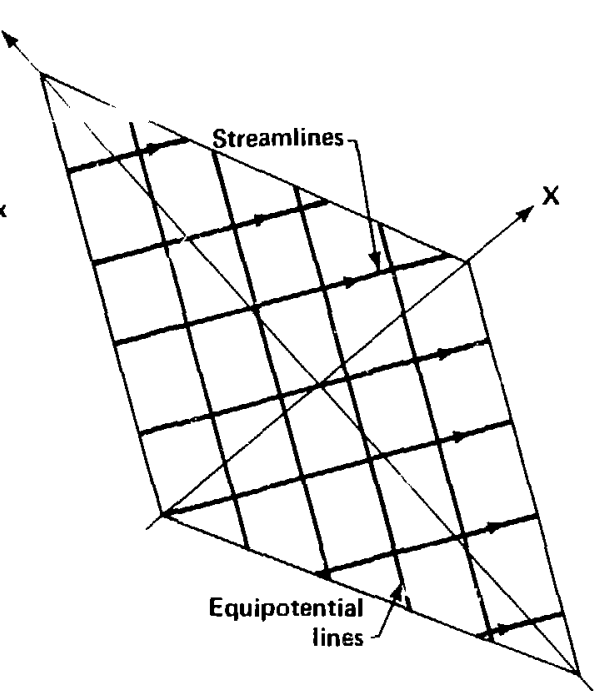

Flow net transformed coordinates

(b)

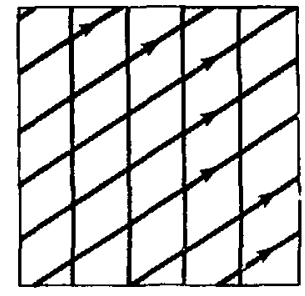

Anisotropic system flow net

(c)

XBL-827.7168 
fluid which has traveled over the same linear distance. This linear length of travel is equal to the length of a streamline that begins on side 2 and ends on side 4 (Figure 3-4c). The liwear travel length is constant within the cross-hatched area between sides 2 and 4 in Figure 3-5. Consequently, measurements of mechanical transport can be conducted in this cross-hatched zone called the test section. Thus, once the hydraulic boundary conditions have been established as illustrated in Figure 3-4a, measurements of mechanical transport can be made within the cross-hatched area shown in Figure 3-5.

\subsection{EXECUTING THE SET OF TRACER EXPERIMENTS TO MEASURE DIRECTIONAL MECHANICAL TRANSPORT}

This section describes the procedure for executing a set of tracer experiments that will allow evaluation of directional mechanical transport for anisotropic porous media. The first step in this procedure sets up a tracer experiment. This step is initiated by selecting a particular orientation of the porous medium to conduct the tracer experiment (Figure 3-6a). Next, the hydraulic boundary conditions shown in Figure 3-4a are applied to a flow region aligned in this direction to create the desired uniform flow field.

In the second step, the tracer experiment is performed by monitoring the detailed movement of fluid with: 7 the cross-hatched test section shown in Figure 3-5. This tracer expeziment is numerically simulated in this research, but the same task may be pefformed experimentally by instantaneously injecting non-diffusive, dyed water uniformly across side 2 of the flow region as shown in Figure 3-6a. The dyed water will flow to side 4 only if it travals within the test section. The breakthrough curve for the fluid flowing in the test section is then measured by recording the concentration of dyed water or side 4 as illustrated in Figure 3-6a. Steps 1 and 2 constitute the procedure for conducting a single tracer experiment. The measurements made from this tracer experiment correspond to a particular direction of flow $\mathrm{B}_{1}$ (Figure 3-6b).

Step three begins the investigation of the directional nature of mechanical transport for the medium. In this step. the orientation of the porous medium is rotated as shown in Figure 3-6c and a second set of measurements of mechanical transpor is made by receating steps 1 


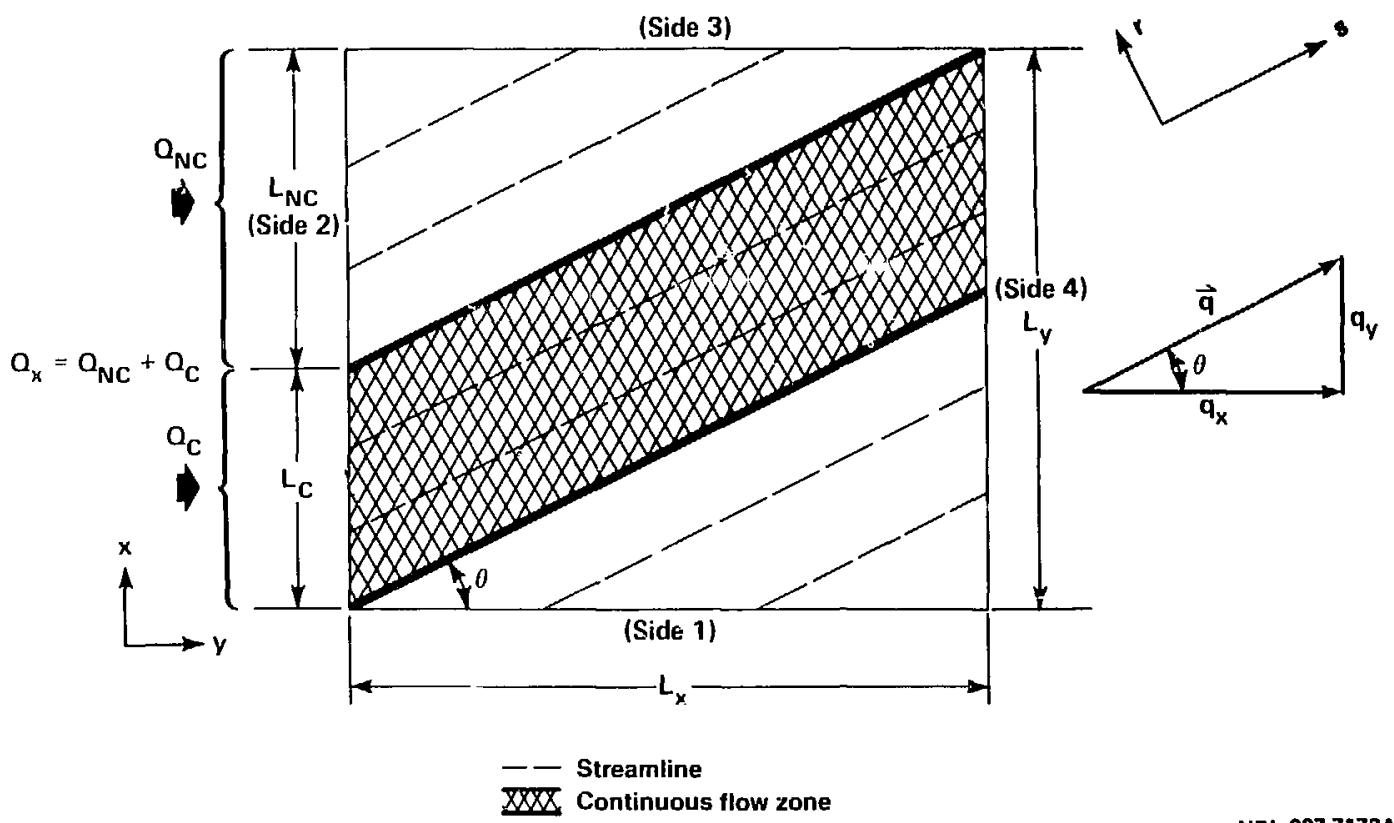

Figure 3-5 Example of Groundwater Flow in an Anisotropic Porous Medium Showing a Cross-Hatched Zone Where Travel Length is Consiant. 


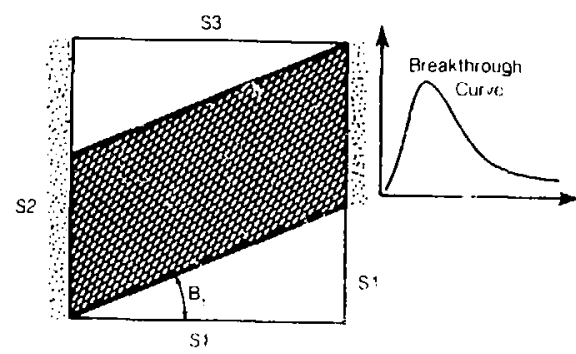

(a)

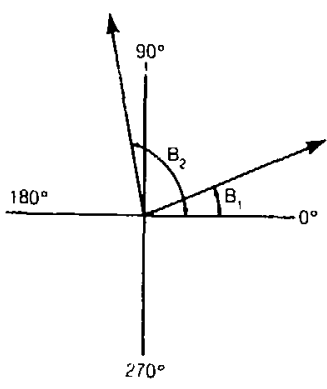

Drechions of Measurements

(b)

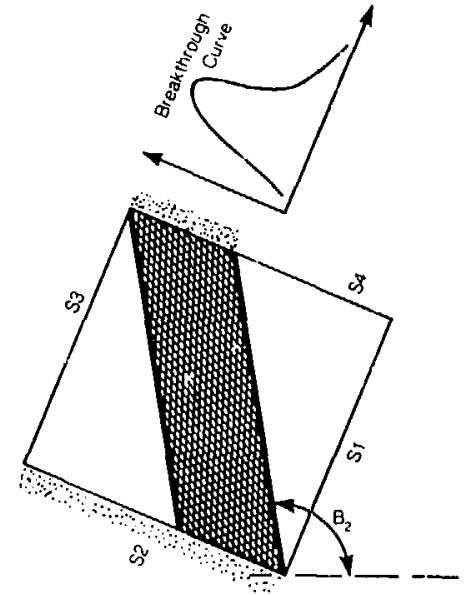

(c)

XBL $841-386$

Figure 3-6 Procedure Used in Conducting a Set of Tracer Experiments to Measure Directional Mechanical Transport for an Anisotropic Porous Medium. 
and 2 for the new direction of flow $B_{2}$ (Figure 3-6b). Next, step 3 is systematically repeated for selected orientations of the porous medium until a representative sample of directional mechanical transport for the medium is obtained. These steps constitute the execution of the set of tracer experiments needed to evaluate directional mechanical transport for an anisotropic porous medium.

\subsection{MEASURING MECHLIVICAL TRANSPORT WITH THE BREAKTHROUGH CIURVE}

The purpose of this seztion is to show how mechanical transport parameters goveming both the advective and isprersive processes in equation 3.6 can be evaluated from the tracer experiments described in the previous section. A tracer experiment is conducted by injecting a pulse of dyed water uniformly across side 2 of the flow region shown in Figure 3-5. At side 4, measurements are made of the concentration of dyed water in relation to time. The time distribution of the outlet concentsation is known as the breakthrough curve or exit-age distribution for the test section. The statistical properties of the breakthrough curve are used to evaluate mechanical transport, as described below.

\subsubsection{Mean of the Breakthrough Curve}

The mean of the breakthrough curve is:

$$
\tau=\frac{\int_{0}^{\infty} \tau \mathrm{cd} \tau}{\int_{0}^{\infty} \mathrm{cd} \tau}=\int_{0}^{\infty} \tau \mathrm{Ed} r
$$

The relationship between the mean of the breakthrough curve and the mean advective rate of movement $\mathrm{q} / \phi_{\mathrm{R}}$ is derived in this section. The area under the normalized breakthrough curve from $t_{1}$ to $\infty$ represents the percent of fluid resicing in the test section longer than $t_{1}$. The volumetric quantity of fluid flowing into the test section in the differential time interval di is Qdt. Thus, the volume of fluid still residing in the test section that first entered $t_{1}$ earlier is:

$$
\text { Qdt } \int_{t_{1}}^{\infty} E d_{T}
$$

The total conductive void volume in the test section is found by integratıng equation 3.7 from t equals 0 to $\infty$ (i.e. for all fluid that enters the test section): 


$$
V_{c}=\int_{0}^{\infty} Q d t \int_{l}^{\infty} E d \tau=Q \int_{c}^{\infty} \int_{1}^{\infty} E d \tau d t
$$

Reversing the order of integration in equation 3.8 yields:

So

$$
V_{c}=Q \int_{0}^{\infty} \int_{0}^{r} E d t d \tau=Q \int_{0}^{\infty} E \tau d \tau
$$

$$
\frac{V_{r}}{Q}=\int_{0}^{\infty} r E d \tau=\bar{\tau}
$$

The average linear velocity is defined as the linear path length of fluid flow divided by the mean travel time. VLIN is an ideal velocity a fluid particle would have if it were constrained to move only in a straight path in the direction of flow:

$$
\mathrm{VLIN}=\frac{\mathrm{L}}{\bar{t}}=\frac{\mathrm{QL}}{\mathrm{V}_{\mathrm{c}}}=\frac{\mathrm{gAL}}{\mathrm{V}_{\mathrm{c}}}=\frac{\mathrm{gV}}{\mathrm{V}_{\mathrm{c}}}=\frac{\mathrm{q}}{\phi_{\mathrm{R}}}
$$

The mean advective rate of movement will be deter.nined fror tracer experiments in this study because rock effective porosity is normally difficult to evaluate directly from laboratory experiments and because transport parameters should be predicted whenever possible from transport data (i.e. breakthrough curve).

The relationship in equation 3.7 assumes that the transpor of all paricies is characterized by a single breakthrough curve. In this equation, $Q$ represents all fluid particles entering the tes. section per unit time and the breakthrough curve represents the fivbability distribution for residence in the test section for every particic. Since the time of residence in the test section for all particles is controlled by a single exit age distribution, fluid transport is homogeneous. Thus, $\phi_{R}$ will equal q/VLIN when the transport of fluid particles is homogeneous.

Field and numerical studies indicate that transpor may be inhomogeneous for homogeneous porous media. Childs et al. (1974) and Hoenn and Roberts (1982) presenie.J field evidence of inhomogeneous transport in homogeneous porous media. Childs et al. used a detailed three-dimensional $: y$ : $y$ sampling points to monitor the movement of waste plumes in an operational homogeneous, sand aquifer. The measured concentration patterns showed that the waste plumes bifurcated, and moved along preferred pathways of travel that were not aligned in the di-artion of the regional flow. The conventional singic plume model could not accurately characterize the pollutant mitration and there was no indication as to the cause for the 
bifurcation of the waste plumes. Th: study of Hoehn and Roberts (1982) found that in a vertically-homogeneous aquifer a two-domain model was needed to simulate transport. The domains in their model consisted of two separate zones (no interaction between zones) with vastly differing rates of movement. Hoehn and Roberts stated that no geological evidence supported the use of the tw'-domain model and noted that the two domains may be distinguishable only at the microscopic level.

Smith and Schwartz (198Ia) conducted numerical mass transport studies for statisticallyhomogeneous porous media which were reviewed in section 2.2. In their Monte Carlo study, it was genirally observed that, in a given realization, the bulk of the mass migratid along a definite path. This connected path linked together the high-pimseable elements within the problem domain where the transport velocities were large. In some cases, the effects of the preferred paths of travel were clearly evident by bimoda! breakthrough curves.

Thus, the following conclusions can be made. The actual rate of advection is controllec by the microscopic velocity field within the pores. If regions of vastly differing rates of movement or large variations in pore velocities exist in the medium, then transport is inhomogeneous. These contrasting domains of transport may be distinguishable only at the microscopic level.

The effect of inhomogeneous transport on the mean advective rate of transport is demonstrated by considering a test section consisting of two separate transport domains: a slow zone of movement and a fast zone of muvement. The flow rate through the slow zone is QS. The particles flowing into the slow zone will prociuce a different breakthrough curve than the paricles flowing into the fast zone. The mean of the breakthrough curve will be larger in the slow zone than in the fast zone, and the composite breakthrough curve for the test snction is the sum of the breakthrough curves for the fast and slow zones. The total conductive voic voiume is given by.

$$
V_{c}=Q S \int_{0}^{\infty} E_{S \tau} d \tau+(Q-Q S) \int_{0}^{\infty} E_{F \tau} d \tau
$$

So. 


$$
\frac{\mathrm{q}}{\phi_{\mathrm{R}}}=\frac{\mathrm{L}}{\frac{\mathrm{QS}}{\mathrm{Q}} \int_{0}^{\infty} E_{S \tau} \mathrm{d} \tau+\frac{(\mathrm{Q}-\mathrm{QS})}{\mathrm{Q}} \int_{0}^{\infty} E_{\mathrm{F} \tau} \mathrm{d} \tau}
$$

The mean of the composite breakihrough curve $\left(E=E_{S}+E_{F}\right)$ is:

$$
\bar{t}=\int_{0}^{\infty} \tau\left(E_{S}+E_{F}\right) d \tau
$$

which is not equal to the denominator in equation 3.10. Therefore, the average linear velocity will not equal $q / \phi_{R}$ if prefer atial paths of movement exist in the test section.

The hydraulic effective porosity is detined to provide a general way of relating the actual rate of transport VLIN to the flow parameter $\mathrm{q}$ :

$$
\phi_{\mathrm{H}}=\frac{\mathrm{g}}{\mathrm{VLIN}}
$$

In porous media transport models, the hydrauic effective porosity is usually assum:d to be equal to $\phi_{\mathrm{R}}$ or $\phi$. The rock effective porosity is a stable rock property that does not vary with direction. The relationship indicated above is valid when transport is homogeneous. Field and numerical studies indicate that transport occurs along preferred paths of travel. When transport is inhomogeneous, the hydraulic effective porosity may deviate from the rock effective porosity. The deviation will increase as the number and size of the microscopic zones of contrasting movement increase. This research will investigate the directional nature of the hydraulic effective porosity for anisotropic media.

\subsubsection{Variance of the Breakthrough Curve}

The type of tracer experiment chosen in this research was carefully jelected such that Fickian longitudinal mechanical transport can be evaluated from the variance of the breakthrough curve. The first objective of this section is to prove that longitudinal mechanical transport can be analyzed for the tracer test chosen in this study by developing the relationship for longitudinal mechanical transpor. The second objective of this section is to show how the variance of the breaktirrough curve can be used to evaluate longitudinal mechanical transpon.

\subsubsection{Longitudinal Dispersion for Pure Mechanical Transport}

The equation governing longitudinal mechanical transpon is derived in this section. It 
will be shown that the mechanical dispersive flux can be characterized by a single, measurable dispersion coefficient. By computing this coefficient, the directional nature of longitudinal mechanical transport can be evaluated from the set of tracer experiments discussed in sections 3.5 and 3.6.

Laminar flow is considered within the porous medium. Laminar flow is characierized by the movement of fluid in laminas or layers, one layer flowing smootbly over the adjacent layers. The streamlines in Figure $3-4 \mathrm{c}$ which are parallel in the flow region indicate the mactoscopic airection of flow. The flow paths of the fluid elements are dictated by the streamlines and cannot cross over one another because the flow is laminar. The fluid particle paths will deviate about the mean flow direction due to the random geometry of the pores. However, the constraint imposed because the streamlines are colinear signifies that the mean direction of mover ent must be in the direction of flow. Suppose a flow region existed in which the paricle paths diverged in many directions such as for flow region b in Figure 3-7. If thi ; flow region is pari of a regional homogeneous, anisotropic medium in which the hydraulic gradient is constant, as hown in Figuie 3-7, the directir ns of flow would vary in the adjacent flow regions. However, this flow situation cannot exist as the direction of flow is constant when the hydraulic gradient is constant in a homogeneou: madium.

The fact that the macrossopic directior. nr movement for all fluid particles is in the direction of flow signifies that there can be no ne: transport of fluid perpendicular to the direction of now. Applying this orinciple to the test section in Figure 3-5, the Fickian mechanical dispersive flux is:

$$
\left[\begin{array}{l}
m_{3} \\
m_{r}
\end{array}\right]=-\left[\begin{array}{ll}
M_{11} & M_{12} \\
M_{21} & M_{22}
\end{array}\right] \nabla c
$$

with

$$
m_{1}=-M_{11} \frac{\partial c}{\partial s}-M_{12} \frac{\partial c}{\partial r}
$$

and

$$
m_{r}=-M_{21} \frac{\partial \varepsilon}{\partial s}-M_{22} \frac{\partial c}{\partial r}=0
$$

Equation 3.13 yields 


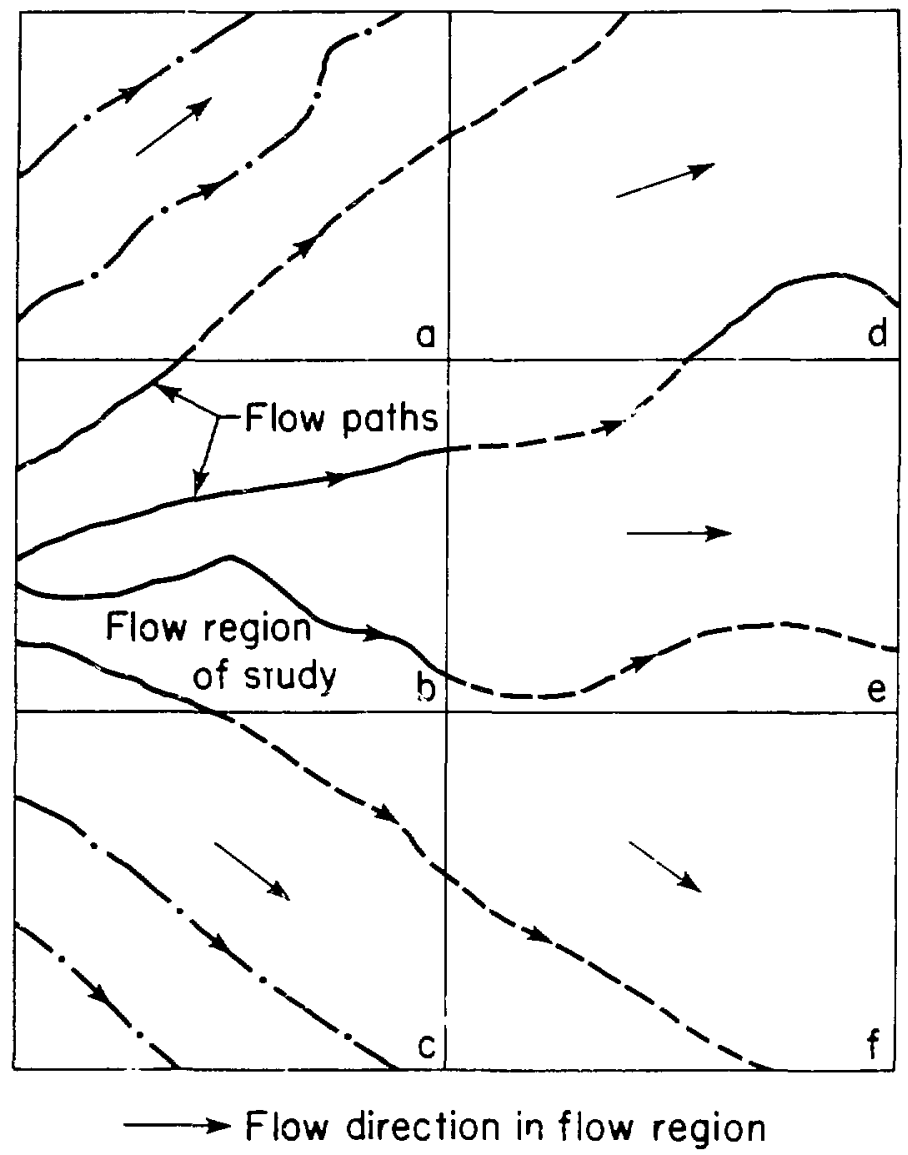

XBL-829-4507

Figure 3-7 Diverg snce of Flow Paths in Fiow Regions Within an Inhomogeneous Regional Flut system. 


$$
-\frac{\mathbf{M}_{21}}{\mathbf{M}_{22}}=\frac{\frac{\partial c}{\partial \mathbf{r}}}{\frac{\partial c}{\partial s}}
$$

Velocity is non-zero only in the s-direction such that:

$$
M_{21}=\sum_{m, n} \alpha_{21 \operatorname{mn}} \frac{v_{m} v_{n}}{V L I N}=\alpha_{2111} \text { VLIN }
$$

and

$$
\mathrm{M}_{22}=\alpha_{2211} \mathrm{VLIN}
$$

Equation 3.14 can be expressed using equations 3.15 and 3.16 as:

$$
-\frac{\alpha_{2111}}{\alpha_{2211}}=\frac{\frac{\partial c}{\partial r}}{\frac{\partial c}{\partial s}}
$$

Substituting for $\frac{\partial c}{\partial r}$ from equation 3.17 into equation 3.12 yields:

$$
\mathrm{m}_{\mathrm{g}}=-\mathrm{M}_{11} \frac{\partial \mathrm{c}}{\partial \mathrm{s}}+\mathbf{M}_{12} \frac{\alpha_{2111}}{\alpha_{2211}} \frac{\partial \mathrm{c}}{\partial \mathrm{s}}
$$

or

$$
m_{1}=-\left[\alpha_{1111}-\frac{\alpha_{1211} \alpha_{211}}{\alpha_{2211}}\right] \operatorname{VLIN} \frac{\partial c}{\partial s}
$$

Incorporating the symmetric relationship of equation 3.4 yields

$$
m_{3}=-\left[\alpha_{1111}-\frac{\left(\alpha_{1211}\right)^{2}}{\alpha_{2211}}\right] \operatorname{VLIN} \frac{\partial c}{\partial s}
$$

The bracketed term in equation 3.18 is a constant, and thus, equation 3.18 may be written:

$$
\mathrm{m}_{1}=-\alpha_{\mathrm{L}} \operatorname{VLIN} \frac{\partial \mathrm{c}}{\partial \mathrm{s}}=-\mathrm{M}_{\mathrm{L}} \frac{\partial \mathrm{c}}{\partial \mathrm{s}}
$$

Note that for an isotropic porous medium, $\alpha_{1121}$ equals zero so that

$$
\alpha_{L}=\alpha_{1111}=\alpha_{1}
$$

where $\alpha_{1}$ is the longitudinal geometric dispersivity for an isotropic porous medium defined in section 3.4. Substituting the expression for $\vec{m}$ into equation 3.6 yields the equation governizg Fickian longitudinal mecharical transport:

$$
\frac{\partial c}{\partial t}+\operatorname{VLIN} \frac{\partial c}{\partial s}=M_{L} \frac{\partial^{2} c}{\partial s^{2}}
$$

Equation 3.19 is classified as an axial dispersed plug flow model because it is one-dir. isional, a function only of the longitudinas variable s. Equation 3.19 shows ihat it is not necessary to 
directly determine all 36 independent components of $|\alpha|$ to evaluate longitudinal dispersion for pure Fickian mechanical transport. $M_{l}$ can be evaluated in a tracer experiment even though the three independent components of $|\alpha|$ which are functions of $M_{L}$ cannot be individually evaluated. The directional nature of $\alpha_{L}$ will be investigated for anisotropic media in this research.

\subsubsection{Eraluation of the Longitudinal Mechanical Dispersion Coefficient}

The longitudinal mechanical transport coefficient $\mathrm{M}_{\mathrm{L}}$ can be calculated from the variance of the breakthrough curve based on some useful formulas derived by Van der Laan (1958) for the moments of this curve. The problem domain considered by Van der Laan consisted of an entrance and exit section whose properties (i.e. VLIN and $M_{L}$ ) were independent from those of a middle, test section. Van der Laan solved a number of transport problems by varying the boundary conditions imposed on the problem domain. In the tracer experiments considered in this research, dyed water is injected and carried across the entrance section/test section interface by the bulk flow. There can be no dispersion upstream of this interface. Within the test section, mechanical transport is governed by equation 3.19. At the test section/exit section interface, the concentration of dyed water is measured. Then, the fluid is advected downstream in the exit section. Van der Laan analyzed this particular problem using Lapiace transformations. He found that the Laplace transform for concentration is a fairly complex expression, and hence, its inverse transformation back into real space was not performed. Fortunately, the moments of the breakthrough curve can be determined without Ferforming this transformation. The variance of the breai:througn curve. a function of the first and second moments. is related to $M_{2}$ in the following way:

$$
\frac{\sigma^{2}}{T^{2}}=2 \operatorname{Pe}-2 \operatorname{Pe}^{2}\left[1-\mathrm{e}^{(-1 / P e)}\right]
$$

where $P e=\frac{M_{L}}{(V L I N) L}$. Thus, $M_{L}$ can be implicitly solved from the variance of the breakthrough curve. The $\log$ of Pe versus $\frac{\sigma^{2}}{t^{2}}$ is shown in Figure 3-8. Note that $\frac{\sigma^{2}}{t^{2}}$ can never exceed unity. 


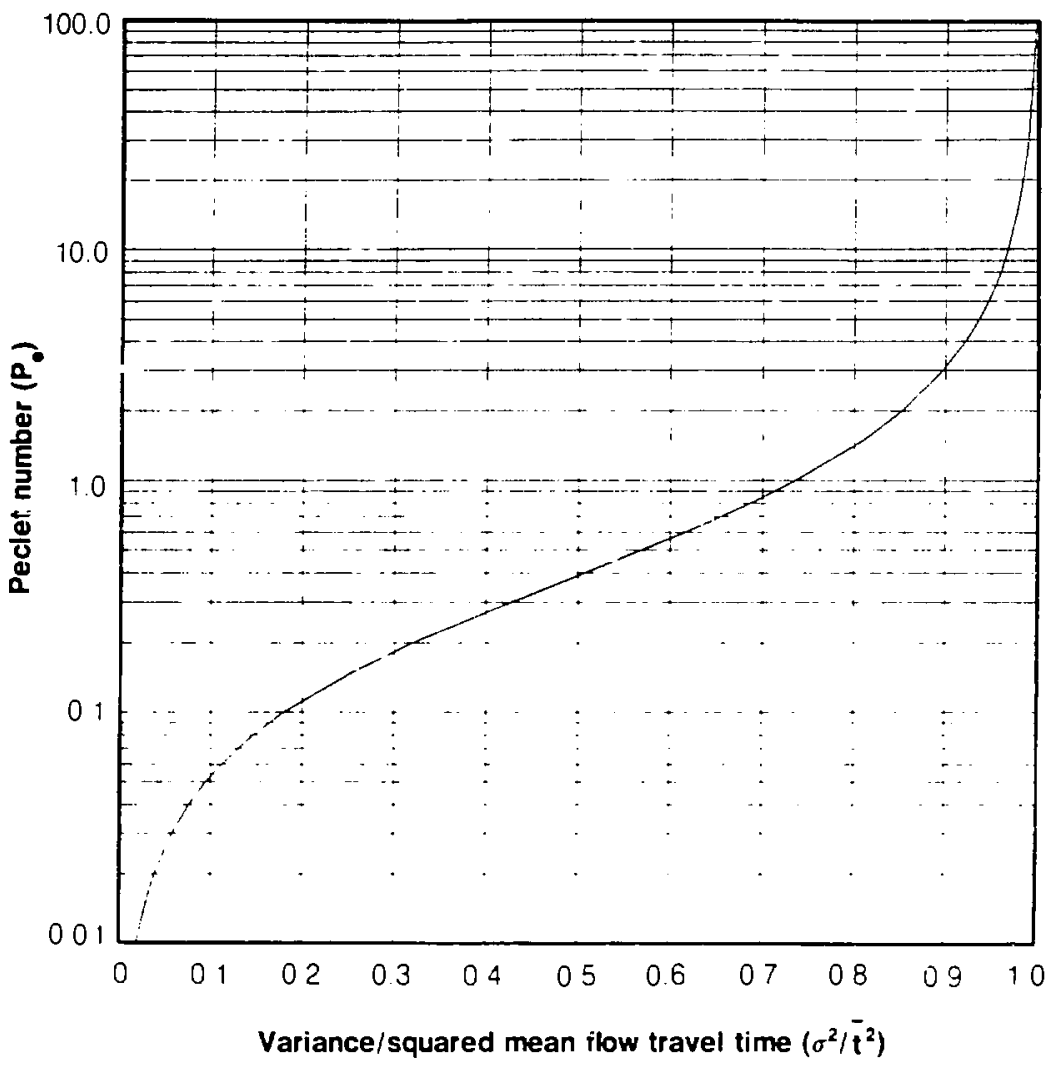

XBL 838-553

Figure 3-8 Semilog Plot of Peclet Number Versus the Ratio of the Variance of th: Breakthrough Curve to the Sqt ared Mean Travel Time. 
Figure 3-9 shows three breaktluough curves for different values of Pe that were constructed using numerical methods (Levenspiel (1972); Yagi and Miyauchi (1953)). The ordinate of these breakthrough curves corresponds to

$$
E^{\prime}=\mathrm{Et}
$$

and the coordinate of the breakthrough curves corresponds to the normalized time

$$
\frac{\mathrm{t}-\mathrm{TM}}{\mathrm{SD}}
$$

where TM= $\tau$ and $S D=\sigma$. The notation changes we: made to accommodate the computer graphic capabilities used in this research.

Figure 3-9 demonstrates that as Pe decreases, the peak concentration increases and shifis toward the righe, and the skewness in the breakthrough curve decreases. The breakthrough curves are slowly converging to the Gaussian distribution as Pe decreases. The Gaussian distribution becomes a good approximation of the breakthrough curve when Pe is less than 0.01. For these small values of Pe:

$$
M_{L} \approx 1 / 2 \frac{\sigma^{2}}{\vec{t}^{2}}(\text { VLIN)L }
$$

\subsection{EQUIYALENT POROUS MEDIUM BEHAVIOR}

Up to this point, the porous medium continuum concept has been applied in order to evaluate fluid flow and mechanical transport. Another important objective is io determine if a fracture system can be ureated like an equivalent homogeneous porous medium continuum. The requirements for continuum flow behavior are presented in this se :ion, followed by a discussion of continuum behavior for hydraulic effective porosity.

In a porous medium. Darcy's law makes it possible to evaluate macroscopic iluid flux properties by treating the medium as a continuum. Fluid flow characteristics are analyzed for equivalent porous medium behavior in two ways. First. flow fie!ds created from the boundary conditions shown in Figure 3-4a are individually evaluated for equivalent porous medium behavior. Then, directional flow is analyzed by symethes:zing flow results in different dircctions. In a porous medium. macroscopic directional now characiensucs an be predicted from a unique permeabihty tensor. 
(a)

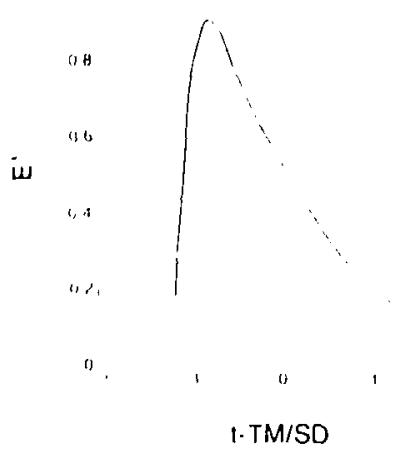

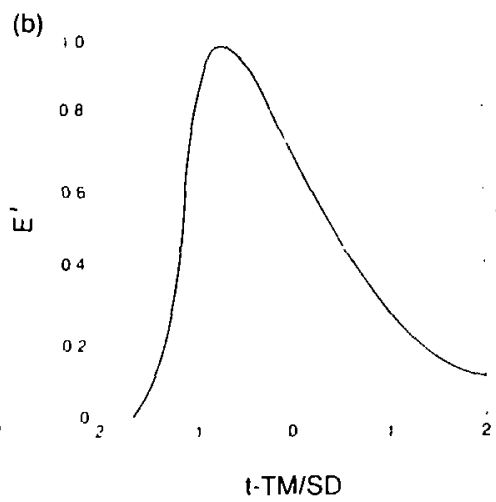

(c) 200

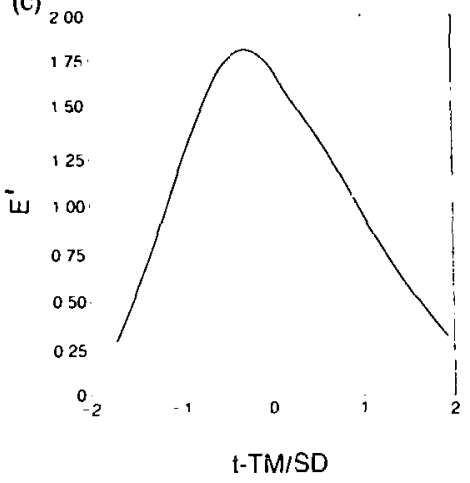

$\stackrel{+\infty}{i}$

TM - inean travel time

$\mathrm{SC}=0$

XBL $83: 27127$

Figure 3-9 Three Breakthrough Curves for a) Fe of 0.5, b) Pe of 0.2, and c) Fe of 0.025. 
Any given flow field must satisfy the following two requirements in order to exhibit equivalent porous medium behavior.

(1) The macroscopic flow field can be predicted by Darcy's law.

(2) The specific discharge is stable and does not fluctuate with the size of the flow region.

As discussed in section 3.5, the flow field for an anisotropic porous medium is characterized by a uniform specific discharge. Because the specific discharge is a vector, the condition of uniformity implies that both its magnitude and $a n_{b}^{*}:$ of flow are constant. The use of macruscopic flow measurements are described below to determine if the magnitude and angle of $\bar{q}$ are constant for the given flow field.

A uniform specific discharge means that parallel cross sections of equal area will have the same total fluid flux flowing across them. This means that in Figure 3-5 the flow rate into side 2 is equal to the flow rate out of side 4 ,

$$
\text { QS2 }=\text { QS4 }
$$

and the flow rate into side 1 is equal to the flow rate out of side 3 ,

$$
\mathrm{QS1}=\mathrm{QS3}
$$

Equations 3.22 and 3.23 constitute a continuity test to eval' 'ate if the magnitude of the specific discharge is constan:.

Two approaches are used to determine the angle of flow. Both approaches produce the same an -le of flow if the specific discharge is uniform. In the first approach, the total flow rates into the sides of the flow region are used to compute the components of the specific discharge is the direction of the hydraulic gradient, and in the direction perpendicular to the gradient from whici angle of flow is calculated. This calculation is not jased on the path of the fluid. In the second approach, the conditions of the uniform flcw field are used to compute angle of flow. As sho - in Figure 3-5, the amount of fluid entering side 2 that exits on side 4 can be uxed to compute ans,le of flow. The remaining nuid entering side 2 must exit on side 3 .

The first me:hod of de:ermining the angle of flow can be expressed mathematically using the two components of the specific discharge: 


$$
\bar{q}=q_{x} \hat{\imath}+q_{y} \tilde{\jmath}
$$

or referring to Figure $3-5$,

$$
\theta=\tan ^{-1} \frac{q_{y}}{q_{x}}=\text { ANFD }
$$

For the flow region in Figure 3-5, the specific discharge in the $\mathrm{x}$ direction is obteined by summing the flow rate into side 2 from the individual flow channels and dividing by $L_{y}$. Similarly, the total flow rate into side 1 divided by $I_{x}$ determines $q_{y}$.

The second method of determining the angle of flow is based on the uniformity of the specific discharge. For the anisotropic medium in Figure 3-5, a certain quantity of the fluid flix, $\mathrm{Q}_{\mathrm{NC}}$, flowing into side 2 must exit on side 3. Thus,

$$
\mathrm{Q}_{N C}=\mathrm{QS3} \text {. }
$$

and

$$
q_{y}=\frac{Q S 3}{L_{x}}=\frac{Q_{N C}}{L_{x}}
$$

Substituting $q_{y}$ into equation 5 yields:

$$
\theta=\tan ^{-1} \frac{Q_{N C}}{q_{x} L_{x}}=\text { ANFC }
$$

This relationship is also obtained directly from the uniformity of the flow field. The cross-hatched area in Figure 3-5 designates the zone in which the fluid flows continuously from sides 2 to 4 . Since the speciñc discharge is uniform, the following relationships hold on side 2:

$$
\frac{\mathrm{Q}_{\mathrm{C}}}{\mathrm{L}_{\mathrm{C}}}=\frac{\mathrm{Q}_{\mathrm{NC}}}{\mathrm{L}_{\mathrm{NC}}}=\frac{\mathrm{Q}_{\mathrm{x}}}{\mathrm{L}_{\mathrm{y}}}
$$

which means

$$
L_{N C}=\frac{Q_{N C} L_{C}}{Q_{C}}=\frac{Q_{N C} L_{y}}{Q_{x}}=\frac{Q_{N C}}{q_{x}}
$$

The relationship for the angle of flow is:

$$
\tan \theta=\frac{\mathrm{L}_{\mathrm{NC}}}{\mathrm{L}_{\mathrm{x}}}=\frac{\mathrm{Q}_{\mathrm{NC}}}{\mathrm{L}_{\mathrm{x}} \mathrm{Q}_{\mathrm{x}}}
$$

so

$$
\theta=\tan ^{-1} \frac{Q_{N C}}{q_{x} L_{x}}
$$

which is the sorre expression as equation 3.25 . 
Equation 3.26 is the second method of computiag the angle cf flow. The evaluation of ANFD and ANFC constitutes the angle of flow test. The direction of the specific discharge is a constant if ANFD is equal to ANFC.

The stability of the specific discharge, the second requirement for a flow field to exhibit porous medium behavior for a particular direction of flow, is tested by slowly increasing the size of the flow region and measuring the specific discharge. Initially, the specific discharge may be expected to fluctuate significantly. In small flow regions, the number of channels (fractures) may be too small so that the flow region does not behave like a representative elementary volume of a porous medium. However, as the size of the flow region increases, the fluctuations in the specific discharge may sampen out and eventually a stable value may be reached. When the specitic discharge is stable, the flow field behaves bydraulically like an equivalent porous medium.

The above tests can be used to examine equivalent porous medium behavior for a paricular direction of flow. However, Darcy's law also specifies that the flow field in any direction can be predicted by a permeability tensor. If such a tensor exists the square root of permeability in the direction of flow plots as an ellipse. For example, the square root of $\mathrm{K}_{\mathrm{f}} / \mathrm{K}_{\mathrm{y}}$ for the anisotropic porous metium considered in Figure 3-4a is shown in the polar plot on Figure 3-10. Thus, the sinape of the plot of the square root of permeability is the test of whether directional flow for the system behaves like an equivalent porous medium.

To determine equivalent porous medium behavior for transport, one must examine the directional nature of the hydraulic effective porosity. The hydraulic effective porosity is generally assumed to be equal either to the rock effective porosity or to the total porosity in transpor modeling. Since both pornsities are independent of direction in a continuum, the hydraulic effective porosity should be constant in all directions. Thus, the test for equivalent porous medium behavior for transport is to examine the stability of $\phi_{H}$ with direction.

The tortuosity was first introduced wi en researchers were developing the theory relating permeability to the geometric properies of a porous medium. One of the first workers, 


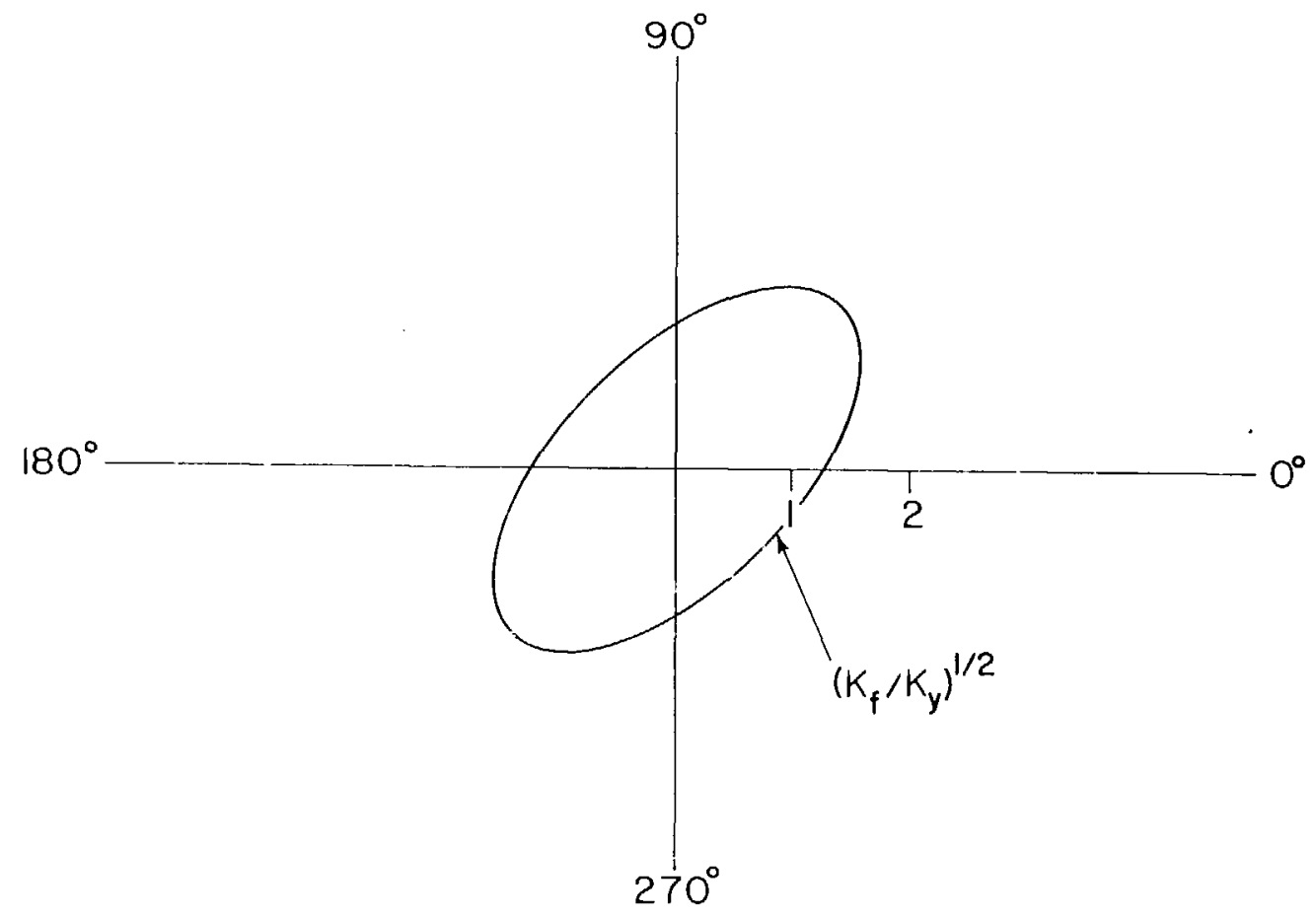

Figure 3-10 Square Ruot of Permeability in Direction of Flow for the Anisotropic Porous Medium Shown in Figure 3-4. 
Kozeny, considered a simple porous medium model consisting of a bundle of capillary tuhes to determine this relationship. The Kozeny-Carman expression for the intrinsic permeability for a medium of straight capillary tubes is:

$$
\begin{gathered}
k=\frac{\phi^{3}}{s_{f} s_{j}} \\
\text { where } s_{f}=32\left(\frac{r_{b}}{d_{i}}\right)^{2} \equiv \text { shape factor }
\end{gathered}
$$

and $r_{b}$ is the conductive volume divided by the wetted surface area, $d_{c}$ is the diameter of a capillary tube, and $S_{3}$ is the specific surface.

Kozeny, also accounted for the fact that fluid paths are nonlinear. The intrinsic permeability in this case (Carman, 1956) is:

$$
\begin{gathered}
k=\frac{\phi^{3}}{S S} \\
\text { where } s=s r^{2}
\end{gathered}
$$

Tortuosity for isotropic porous media has been evaluated both analytically for random oriented flow channels (Haring anc Greenkorn, 1970) and experimentally, by diffusion studies (Collins, iÝó), to be $\sqrt{3}$ and $\sqrt{2}$, respectively. Dullien (1979) stated that $\tau$ shouid normally range between 1 and $\sqrt{3}$.

Tortuosity for anisr:ropic porous media is directionally dependrnt. Anisolropy (Rice et al., 1970) is caused by differences in path lengths of fluid particles and frictional resistance with direction resulting from the asymnicisit shape of grains oriented in a particular direction. Longer paths of flow cause greater frictional resistance which subsequently reduces flow rate. The disectional dependence of $\tau$ v. as show experimentally by Sullivan and Hertel (1940) using a medium of glass wool. Sullivan and Hertel showed that the tortuosity yarallel to the direction of the fibers was much less than $\tau$ measured in the direction perpendicular to the fibers.

Directional permeability from equation 3.27 is caused by variations in $\mathrm{s}$. The shape fartor $\mathrm{s}_{\mathrm{f}}$ exhibits relatively small changes for different geumetrically shaped conduits (Wyllie and Spangler, 1952) and normally falls between 2 and 3. Thus, only slight changes in $s_{f}$ are expected with direction in an anisotropic medium. This means that directional permeability 
strongly reflects changes in tortuosity. Since no known extensive study of directional tortuosity has been conducted, one would expect tortuosity to be inversely proportional to permeability for an equivalent porous medium. 


\section{CHAPTER 4 \\ THE DISCRETE NUMERICAL MOUEL}

\subsection{INTRODUCTION}

A three-stage discrete numerical model written in FORTRAN specifically for the VAX11/780 machines has been developed to evaluate mechanical transport in fracture systems. The first stage of the model is called fracture system generation. The function of this stage is to create a homogeneous, anisotropic fracture system for which u':e directional nature of mechanical transport can be investigated. In this stage, finite-element meshes are constructed for flow regions oriented in difierent directions so that the set of tracer tests used to evaluate directional mechanical transport can be simulated. The second stage is called the hydraulic head calculation. The hydraulic boundary conditions described in section 3.5 are appiled to each flow region and a finite element method is used to calculate the hydraulic head at each fracture intersection within the flow region. The distribution of hydraulic head serves as input to the third stage of the numerical model called mechanical transport simulation. Mechanical transport is modeled in this stage using a new streamtubing technique which traces the detailed movement of fluid flow. At the end of this stage, macroscopic mechanical transport and fluid flow parameters are calculated. Each stage is discussed in detail in this chapter.

\subsection{FRACTURE SYSTEM IENERATION STAGE}

In the first stage of the numerical model, a two-dimensional fracture system is created in an area called the generation region. The procedure used in creating the fracture system was developed by Long (1983). The fractures in the generation region are created one set at a time, and the number of fractures in each set is cont. olled by an assigned areal density (number of frostures per unit area). The geometric parameters required to crerie each fracture are: its length, orientation, aperture, and location in the generation region. This information may be read directly into the computer program (deterministic approach) or may be generated stochastically. In a stochastic generation, each fracture is randomly located in the generation region to 
create a statistically homogensous system. The three remaining geometric parameters are each created by probabilistic simulation. Probabilistic simulation can be conducte: with either the Gaussian, lognormal or exponential probability disiribution. The mean and siandard deviation for the simulated distribution must be read into the computer program.

The stochastic generation of a fracture system consisting of two sets of fractures is illustrated in Figure 4-1. In Figure 4-1a, the size of a square generation region is defined, and the center of the first fracture for set 1 is randomly located in the generation region. This fracture is then assigned an orientation (Figure 4-lb) using probabilistic simulation. Next, the fracture is stochastically assigned a length and an aperture as sbown in Figure 4-1c. The steps shown in this figure from 4-1a to 4-1c are used to create a single fracture. The number of fractures created for set 1 is controlled by its areal density. Each fracture for the first set is generated from the same set of geometric statistics (mean and standard deviation) for length, aperture. and orientation. After all the fractures have been created for set 1 (Figure 4-1d), the fractures for set 2 are created from a new set of geometric statistics. Figure 4-le shows the complete fracture system consisting of two sets of fractures.

Flow regions within the generated fracture network are selected for fluid flow and mechanical transport studies. A flow region may be oriented in any direction as long as it fits within the boundaries of the generation region. A finite-element mesh is constructed fe: each flow region consisting of nodes, which are fracture intersections, and elements, which are fracture segments between nodes. Figure $4-1 \mathrm{f}$ shows a flow region within the generation region oriented at $45^{\circ}$. The finite element mesh for the flow region consists of 21 nodes and 22 elements. The hydraulic boundary conditions described in section 3.5 are applied to each flow region and the hydraulic heads for the nudes located along the bourdary of the flow region are calculated. The finite-element mesh with the prescribed nodal hydraulic heads are stored in a data file. This file is read into the second stage of the computer program. 


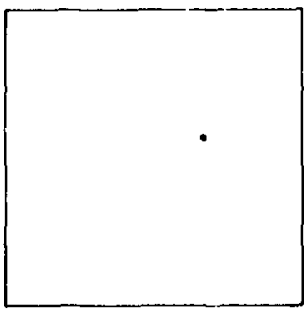

(a) Selection of genera tion region size with center of first fracture for set I randomly located in domain

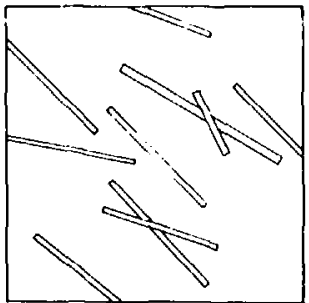

(d) Fractures for set I created

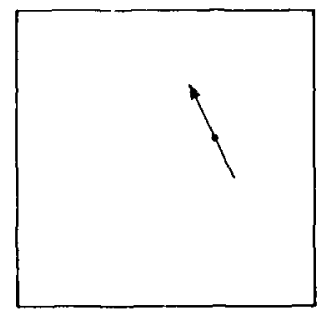

(b) Fracture orientation assigned

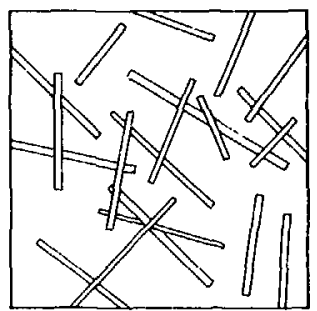

(e) Fractures for set 2 completes generation of fracture system

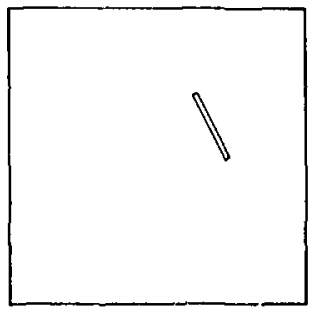

(c) Frocture iength and aperture assigned

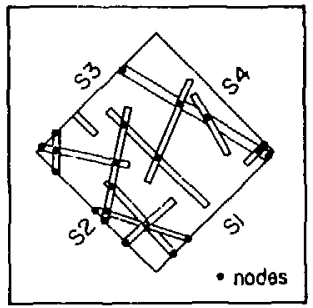

(f) Flow region orient ed at $45^{\circ}$ consisting of 21 nodes ana 22 elements

Figure 4-1 Stochastic Generation of a Fracture System Consisting of Two Sets of Fractures. 


\subsection{HYDRAULIC HEAD CALCULATION}

The steady state hydraulic heads at the nodes are calculated in the second stage using a finite-element technique developed by Wilson (1970). Laminar flow in each fracture is governed by the cubic law ior fracture flow:

$$
Q_{\mathrm{e}}=-\frac{\rho \mathrm{gb}^{3}}{12 \mu} \frac{\mathrm{d} \Phi}{\mathrm{d} \xi}
$$

The finite element method first develops the necessary algebraic equations governing flow within each element by discretizing the derivative for $\Phi$ in equation 4.1. The two equations governing flow into the two ends (nodes) of the element shown in Figure +2 ars:

$$
\text { Node i: } \quad Q_{e i}=\frac{\rho g b^{3}}{12 \mu L_{e}}\left(\Phi_{i}-\Phi_{j}\right)=a_{F}\left(\Phi_{i}-\Phi_{j}\right)
$$

and

$$
\text { Node j: } \quad Q_{e j}=\frac{\rho g b^{3}}{12 \mu L_{e}}\left(\Phi_{j}-\Phi_{i}\right)=a_{F}\left(\Phi_{j}-\Phi_{i}\right)
$$

Equations 4.2 and 4.3 govern flow within a one-dimensional finite element called a line element. These two equations can be expressed in matrix notation as:

$$
\mathbf{a}_{\mathbf{F}}\left[\begin{array}{cc}
1 & -1 \\
-1 & 1
\end{array}\right]\left[\begin{array}{l}
\Phi_{i} \\
\Phi_{i}
\end{array}\right]=\left[\begin{array}{l}
Q_{i j} \\
Q_{e j}
\end{array}\right]
$$

or

$$
\left|C_{\mathrm{e}}\right|\left\{\Phi_{\mathrm{e}}\right\}=\left\{Q_{\mathrm{e}}\right\}
$$

A set of such equations is computed for each element in the flow region. Next, all the sets of equatirns governing flow at the element level are assenıbled into a global set of equations governing fow for the entire region. The matrix form of this global set of equations is:

$$
\left|C_{i} \Phi_{i}=\right| Q \mid
$$

The conductance matrix $[\mathrm{C}]$ is simply calculated by adding all the $2 \times 2$ element submatrices into a square $\mathrm{n} \times \mathrm{n}$ matrix where $\mathrm{n}$ is the number of nodes. The conductance matrix for this problem is symmetric and banded. Both $\{\Phi\}$ and $\left\{Q_{i}\right.$ are $n \times 1$ column vectors of nodal hydraulic heads and prescribed flow rates. respectively. For the boundary conditions considered in section 3.5. $Q \mathbb{Q}$ is the null vector. After the known nodal hydraulic heads along the boundary of the flow region have been implemented into equation 4.5. a direct linear equation solver for symmetric banded matnces is ised to calculate the nodal hydraulic heads. This 


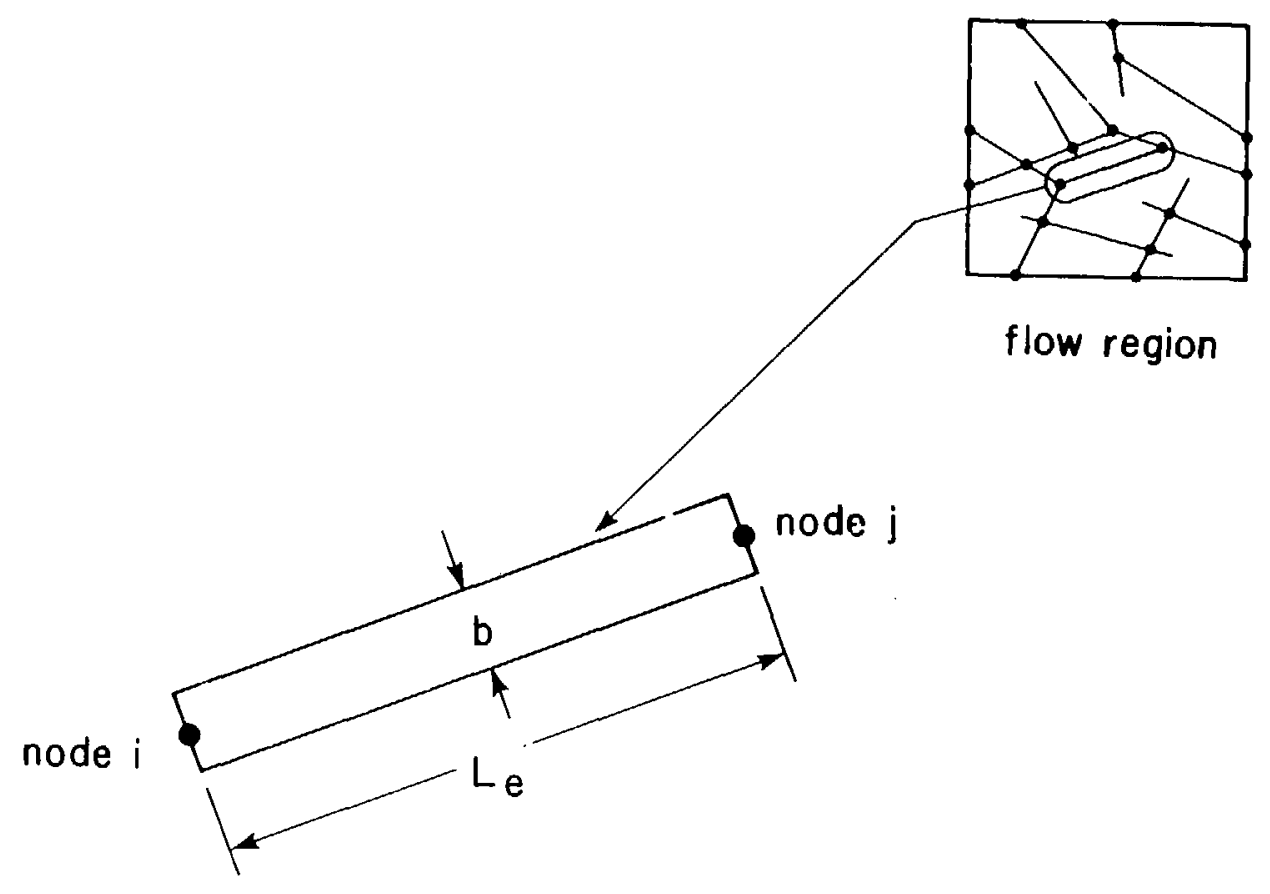


constitutes the second stage of the numerical model.

\subsection{MECHANICAL TRANSPORT SIMULATION}

Mechanical transport is simulated for the elements in the flow region in the final stage of the program. A strea stube is defined as a flow conduit that is bounded by streamlines in which flow rate is constant. The model developed to simulate mechanical transport is used to determine the paths and flow rates for the streamtubes in the fracture network. Once all the streamtube [, aths have been determined, the total travel time from inlet to outlet for the fluid in each streamtube is computed by summing up the residence times in each element along the individual streamtubes. This procedure requires an evaluation of the path, the flow rate, and the width of the streamtube in each element. The time it takes the fluid in a streamtube to travel the length of an element will first be considered. Since fluid flows out of an element to a node, the way fluid flowing into a node exits out of the node will then be discussed.

As mentioned earlier, the flow rate in an element is governed by the cubic law for fracture flow. It can be shown that by solving the Navier-Stokes equation for laminar flow between parallel plates, an expression for the well-known parabolic velocity distribution across a planar element is obtained:

$$
u(\eta)=\frac{\rho g}{\mu} \frac{\mathrm{d} \Phi}{\mathrm{d} \xi}\left[\frac{\eta^{2}}{2}-\frac{\mathrm{b}}{2} \eta\right]
$$

The flow rate in the element is obtained by integrating equation 4.6 across the width of the clement:

$$
\mathrm{Q}_{\mathrm{r}}=\int_{0}^{\mathrm{b}} \mathrm{u}(\eta) \mathrm{d} n=-\frac{\rho g \mathrm{~b}^{3}}{12 \mu} \frac{\mathrm{d} \Phi}{\mathrm{d} \xi}
$$

This is the cubic law for fracture flow. 
The time it takes the fluid in a streamtube to travel the length of an element is given by:

$$
t_{S T}=\frac{L_{T}}{\frac{Q_{S T}}{\eta_{i+1}-\eta_{i}}}
$$

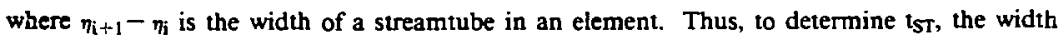
that a streamtube occupies in an element must be computed. Integrating equation 4.6 between $\eta_{\mathrm{i}}$ and $\eta_{\mathrm{i}+1}$ yields:

$$
Q_{S T}=\int_{n}^{m+1} u(\eta) d \eta=\frac{6 Q_{f}}{b^{3}}\left[\frac{b\left(\eta_{i+1}^{2}-\eta_{1}^{2}\right)}{2}-\frac{\left(\eta_{1+1}^{3}-\eta_{i}^{3}\right)}{3}\right]
$$

The width occupied by the streamtube can then be determined from the $N$ rate in the streamtube and the starting coordinate $\eta_{i}$. For example, the node in Figure 4-3 ronsists of three inflowing streamtubes labeled ST1, ST2, and ST3. Streamtube ST2 has a fl $\vee$ rate of 4 units and a staring coordinate, $\eta_{i, 2}$, equal to zero in element $E$. The ending coordinate of ST2, $\eta_{i+1,2}$, is obtained using equation 4.7 . That coordinate then becomes the startin cou dinate for ST3 in element $E$ from which the travel time for ST 3 in element $E$ is determined.

The principle of conservation of mass, and the fact tha! strea lines cannot cross one another in laminar flow, are used to calculate the downstream locatic of inflow streamtubes in outflow elements at a node. Travel times within a node are considered to be negligible. The upper outflow elcment $D$ in Figure 4-3 has a flow rate of three units. The flow into this element must come from element $A$ because if any of the other $t$, s streamtubes flowed into element D, they would have to cross the path of ST1. Using the ame principle. ST 2 mi ust occupy the upper, and ST3 the lower, portion of element E. The order of the streamtubes and the flow rates in each streamtube are recorded for cach outflow ele nent. This information is needed to determine the travel tim: for the flund in each streamtube

The flux in an inflow streamtube can be distribued into more than one outflow element at the node, as is illustrated for STl in Figure 4 . When this arises. the inflow streamtube must be subdivided such that a new streamtube is created for every out?low element that receives any portion of the inflow. For example.ST3 and ST4 are the result of the subdivision 


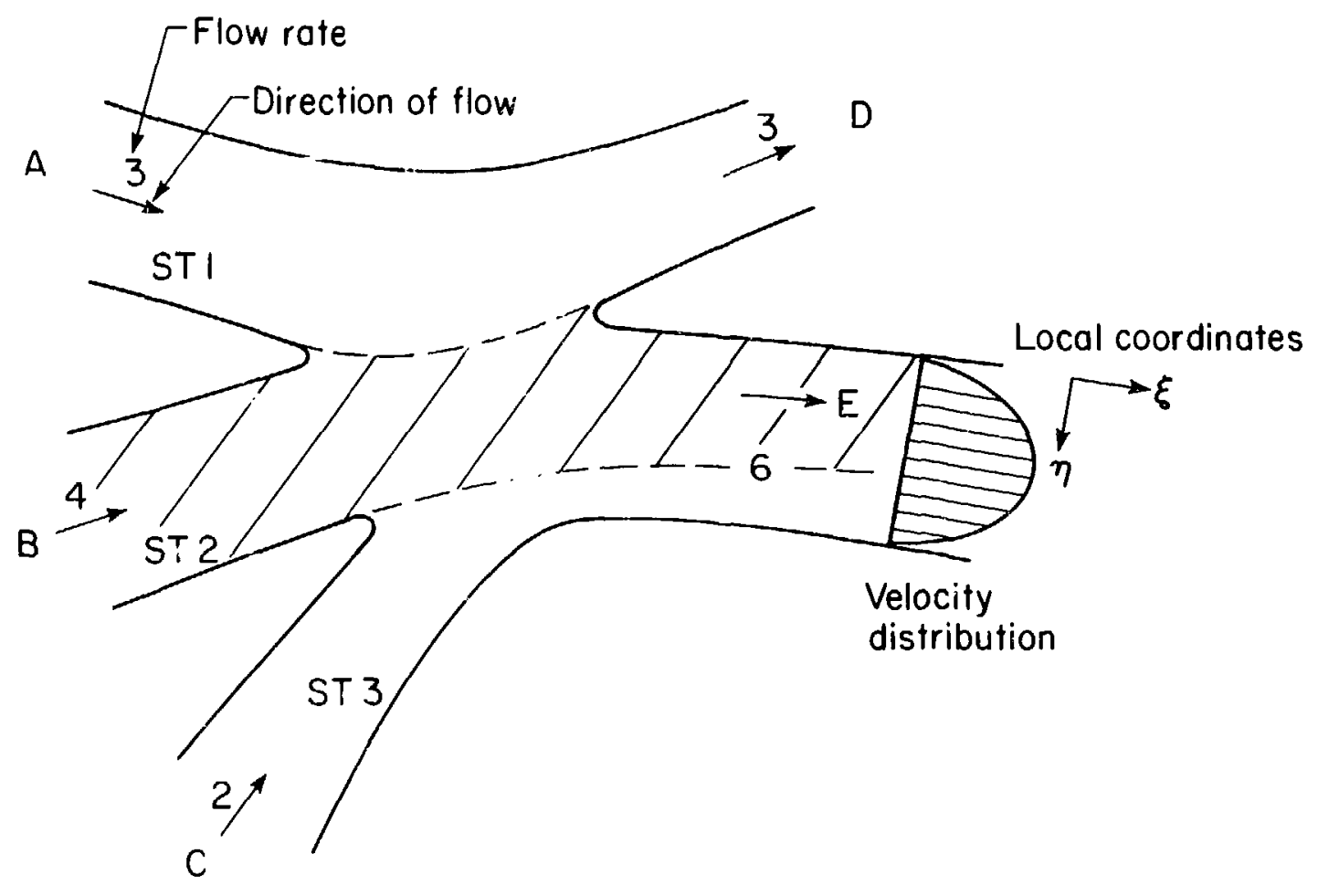

Figure $4-3 \quad$ Redistribution of Streamtubes at a Node. 
(a)

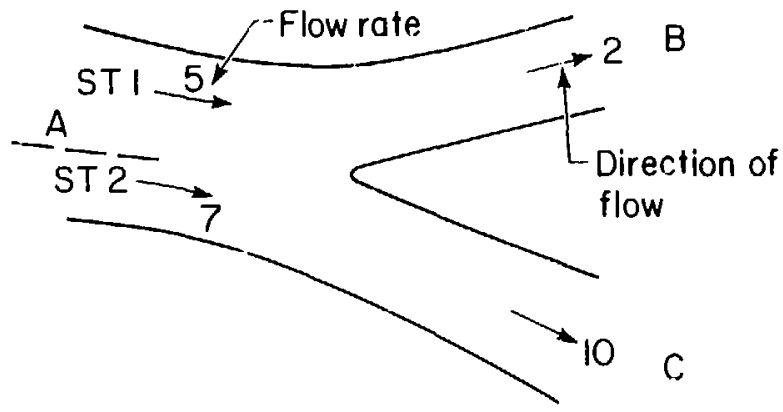

Two inflowing streamtubes (b)

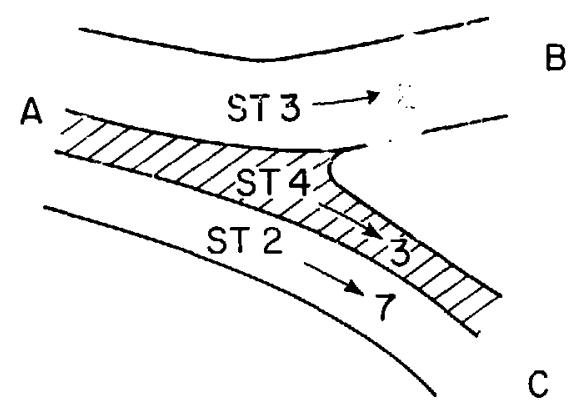

Two new streamfubes created XBL.829-4532 
of the discontinued streamtube ST1. The total travel time to this particular node for the fluid in a new streamtube is determined by backtracking along the path of STI to its orizin.

The first step in tracing the location of streamtubes in the fractur - etwork is to assign a streamtube to every inflow element on the boundary of the flow rogion. This assures that streamtubes exist in every conductive element within the flow region. For example, in the fracture network shown in Figure 4-5, streamtubes have been initiated in elements 1, 2, and 5 . Each assigned streamtube is given a width equal to the aperture of the element it occupies, and a flow rate equal to that in the element.

The program inen proceeds in sequential nodal order determining the outflow streamtubes at asch yode. The outflow streamtubes at a node can only be determined if the streamtubes are known in all inflow elements at the node. If streamtubes do not exist in an inflow element, the inflow element number and the node number are stored in memory. This situation arises when an inflow element at the node under consideration is an outflow element at a highe, numbered jode. The strearitubes in the inflow element at the current node can only be tetermined after proceeding to tike higher node. In Figure $4-5$, node $i$ is the first node examined by the computer program and element 4 is the only ovitlow element at this node. The streamtubes in element 4 can only be calculated if the strearmubes are known in inflow elements 1 and 3. The streamtube in element 1 is known since a streamtube was assigned to this element in the first phase of the streamtubing procedure. However, at this point, the streamtubes in element 3 are unknown. Therefore, element 3 and node 1 are stored in memory and the program proceeds to node 2.

The streamtubes in the outflow elements at a node are delermined when streamtubes exist in all inflow elements. After the streamtubes in all outflow elements have been determined, the program scans the elements stored in memory and removes any element that is an outflow element at the current nude because the streamtubes are known in these elements. If an element is the only one stored for a particular node, then the streamtubes in the outflow elements at that node are determined. For example, at node 2 for $t \mathbf{r}$ racture network in Figure $4-5$. the 


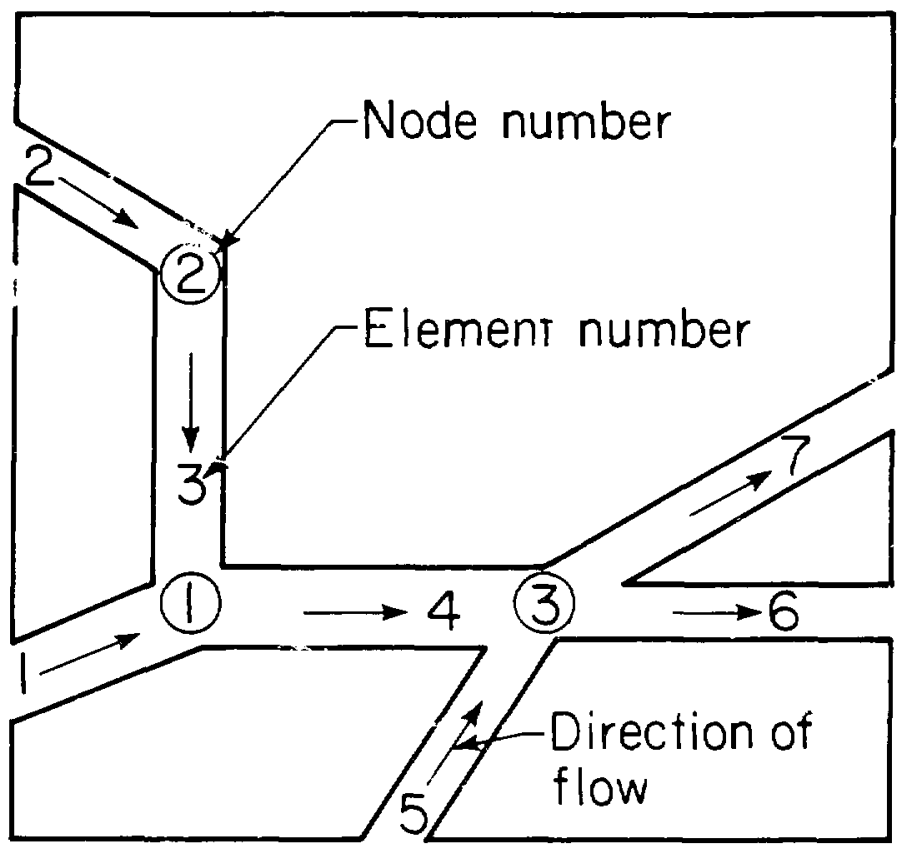

XBL 833-:414 A

Figure 4-5 Fracture Network With Inflow Streamtubes Initiated in Elements 1,2, and 5. 
streamtube for the inflow element 2 is known. The streamtube in the oufiow element 3 can therefore be computed knowing the streamtube in element 2 . The program then removis clement 3 from the list of stored elements because the streamtubs in element 3 has been determined. Because element 3 is the only element stored in memory for node 1, the streamiubes in the outflowing element 4 at node 1 can now be calculated. As the network is scanned in this fashion, the number of streamtubes increases and the width of streamtubes decreases because new streamtubes are being created at nodes.

After the streamtubing procedure has been completed, the program computes the macroscopic fluid flow and mechanical transport parameters listed in Table 4-1.

\section{Table 41. Macroscopic Parameters Calculated in Mechanical} Transport Simulation Stage.

\section{FLUIU FLOW INFORMATION}

Flow into side 2 (QS2)

Flow out of side 4 (QS4)

Flow into side 3 (QS3)

Flow out of side 1 (QS1)

Continuous flow from side 2 to 4 (QC)

Magnitude of srecific discharge (q)

Deviation in flow (DEVF)

Angle of flow based on flow uniformity (ANFC)

Angle of flow based of components of specific discharge (ANFD)

Deviation in angle of flow (DEVA)

Tortuosity $(\tau)$

Breakthrough Curve Statistics

Mean flow travel time (t)

Variance $\left(\sigma^{2}\right)$

\section{Porosity Inismation}

Total porosity of flow reginn $(\phi)$

Rock effective porosity $\left(\psi_{R}\right)$

Hydraulic effective porosity $\left(\phi_{H}\right)$

\section{, Velocity Information}

\section{Average linear velocity (VLIN)}

Mean pore velocity (MPV)

Calculated mean pore velocity (VPORE)

There is no guarantee tha+ a fracture system will behave like an equivalent porous medium and satisfy the continuity criterion discussed in section 3.8. Consequently. the specific 
discharge is defined by recognizing that Darcy's specific discharge represents the average discharge crossing a unit area in the mean direction of flow. The magnitude of the specific discharge is : "s computed by averaging the flux on the sides of the flow region in the following way:

$$
q_{x}=\frac{Q S 2+Q S 4}{2 L_{y}} \text { and } q_{y}=\frac{Q S 1+Q S 3}{2 L_{x}}
$$

with

$$
q=\left(q_{x}^{2}+q_{y}^{2}\right)^{y}
$$

It was shown in section 3.8 that for a porous medium:

$$
\mathrm{QS2}=\mathrm{QS4} \text { and } \mathrm{QS} 1=\mathrm{QS3}
$$

The exact equality indicated by the two equations above will not necessarily hold for stochastically generated fracture systems. The relationship for the fow rate along the sides of the flow region can be written:

$$
\mathrm{QS2}=\mathrm{QS4}+\Delta \mathrm{Q} \text { and } \mathrm{QSI}=\mathrm{QS3}+\Delta \mathrm{Q}
$$

The deviation in flow is defined to measure how well the continuity criterion is satisfied for the flow field within a flow region:

$$
D E V F=\left(\frac{|\Delta Q|}{T O T A L \text { INFLOW }}\right) 100
$$

is small deviation in flow indicates that the continuity criterion is satisfied.

Two methods were presented in section 3.8 to compute the angle of flow. The continuous flow from side 2 to side 4 was used in calculating ANFC by equation 3.25. This method was based on the uniformity of the flow field. In the second method, the two components of the sperific discharge were used in calculating ANFD by equation 3.24. The angle of flow for the specific discharge is thus computed by averaging ANFD and ANFC:

$$
\theta=\frac{\text { ANFD }+ \text { ANFC }}{2}
$$

The deviation in angle of flow is defined to measure how well the flow field within a flow region satisfies the angle of flow criterion:

$$
\text { DEVA }=\mid \text { ANFD - ANFC I }
$$

ANFC will equal ANFD (DEVA $=0$ ) if the fracture system behaves exactly like an equivalent 
purous medium. Thus, DEVA and DEVF are both used to evaluate how well a flow field satisfies the ff: $\mathrm{w}$ requirements for equivale:: oorous medium behavior.

The breakthrough curve is constructed for the fluid that flows continuously between s des 2 and 4 . The mean of this curve is used to compute VLIN in the following way:

$$
\text { VLIN }=\frac{L}{\bar{t}}=\frac{L_{x}}{\cos \theta \bar{t}}
$$

Next, VLIN is used to compute the hydraulic effective porosity as:

$$
\phi_{\mathrm{H}}=\frac{\mathrm{q}}{\mathrm{VLN}}
$$

The variance of the breakthrough curve can be used to corr. ic the Fickian longitudinal mechanical transport coefficient from equation 3.20 , once VLIN and $\mathrm{L}$ have been computed.

The mean pore velocity is the average microscopic velocity within the pores and is expressed mathematically as:

$$
\text { MPV }=\frac{1}{V_{c}} \int_{V_{c}} v^{d v_{c}}
$$

A commonly accepted relationship associating the mean pore velocity with the specific discharge is the Dupuit-Forchheimer assumption (Scheidegger, 1960):

$$
M P V \approx q / \phi_{R}
$$

The rock effective porosity is computed by summing the volume of all conductive elements within the flow region and then dividing this term by the total volume. Fc: homogeneous transport, $q / \phi r_{i}$ is equal to VLIN, which means that the Dupuit-Forchheimer yield :

$$
\mathrm{MPV} \approx \mathrm{VLIN}=\frac{\mathrm{L}}{\overline{\mathrm{t}}}
$$

Fuid particles travel along nonlinear paths in a porous medium such that the mean pore velocity is larger than the average linear velocity. Consequently, a better estimate of MPV is provided in this study by VPORE. VPORE is computed using the tortuosity to account for the nonlinearity of fluid flow in the following way:

$$
\text { VPORE }=\frac{L_{\mathbf{z}}}{\bar{t}}=\tau \text { VLIN }
$$

Tortuosity is computed as:

$$
\tau=\frac{\sum_{i=1}^{N C} Q_{S T i} L_{S T i}}{Q_{C} L}
$$




\section{CHAPTER 5 \\ INVESTIGATION OF CONTINUOUS \\ FRACTURE SYSTEMS}

\subsection{INTRODUCTION}

Two regular continuous systems of infinitely long fractures were initially studicd for two primary reasons. First, continuous fracture systems behave hydratilicaliy as equivalent porous media with flow properties that can be analytically computed (Parsons [1966|, Snow 1969'। The study of fracture systems with known continuum flow behavior can serve to demonstrate the tests developed to identify such behavic Second, the possible directional dependence of mechanical transport can be investigated for anisotropic fracture systems in which the vaid region is totally connected. Such fracture systems simplify an understanding of the results because dead-end zones are excluded from the void region.

\subsection{CONT1NUOUS SYSTEM WITH TWO SETS OF CONSTANT-APERTURE FRACTURES}

In the first investigation of networks with continuous fractures, the system consisted of two sets of parallel fractures oriented at $0^{\circ}$ and $30^{\circ}$, as illustrated in Figure $5-1$. All fractures had an aperture of $0.002 \mathrm{~cm}$ and the spacing between fractures was a constant value of $10 \mathrm{~cm}$.

Several different sized flow regions were analyzed to investigate the requirement that a fracture system nat exhibits porous miedium behavior should have a specific discharge that remains stable. This study was conducted by varying the size of seyeral square flow regions oriented at $0^{\circ}$ and observing if the numerical solutions for $\theta$ and $\theta$ converge to their theoretical values as the size increases. The theoretical solutions for $q$ and $\theta$ apply to flow regions of infinite dimensions. However, only finite-sized flow reguons can be created using numerical modeis. Thus, the difference between the theoretical and numerical soltions for $q$ and $A$ should decrease as siza : icreases. 


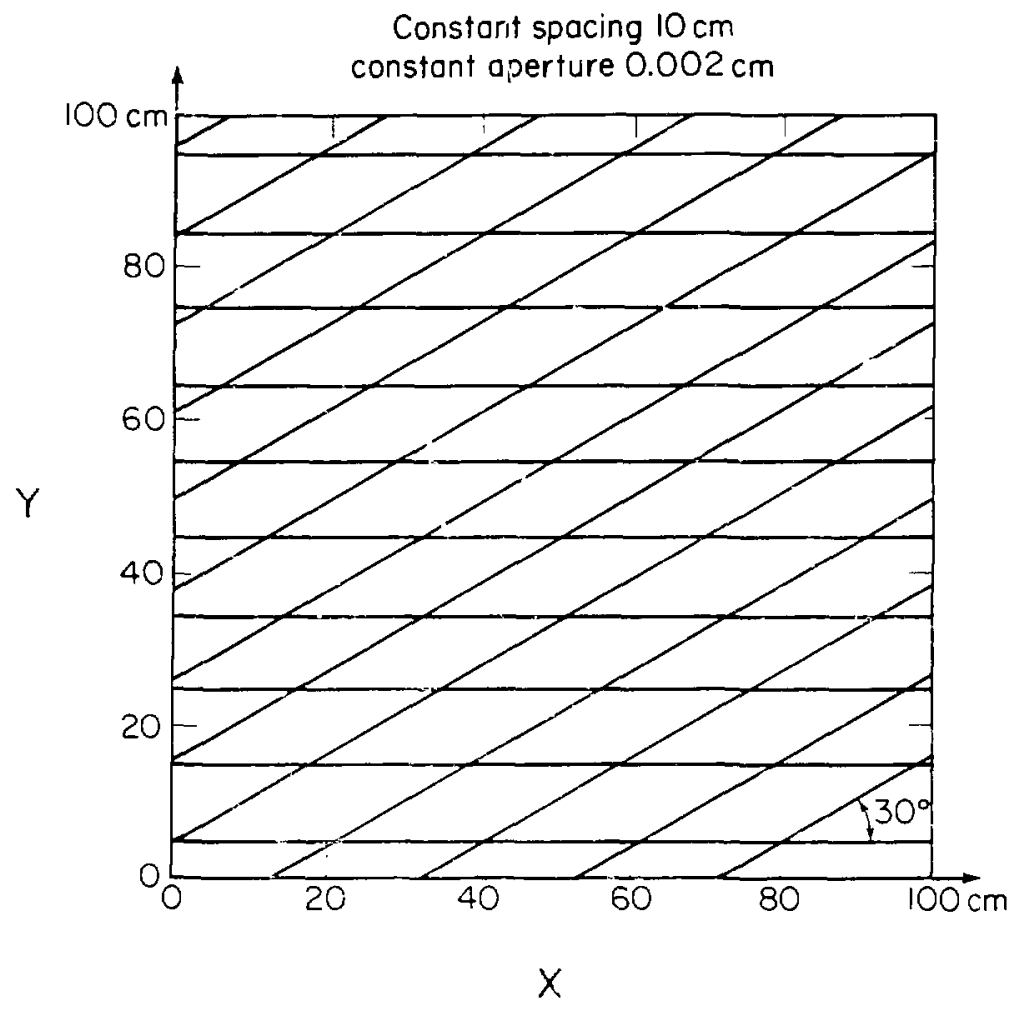

XaL.929-4542

Figure 5-1 Fracture System With Two Sets of Parallel. Continuous, and Constant Aperture Eractures. 
Figure 52 shows how the ratio of actual to theoretical specific discharge (q/qT) approaches unity as the size of the flow region increases. Porous medium equivalence is also evident from the results for angle of flow. As shown in Figure 5-2, the deviation between actuai and theoretical angles of flow $\left(\left|\theta-\theta_{\mathrm{r}}\right|\right)$ is negligible for flow regions larger than $200 \times 200 \mathrm{~cm}$. Further evidence that this size fracture network exhibits porous medium behavior can be obtained from the ratio of actual to theoretical porosity $\left(\phi / \phi_{\mathrm{T}}\right)$, which has also been plotted in Figure 5-2. Thus, the stability requirement is met for this particular fracture network when the size of the flow region is $200 \times 200 \mathrm{~cm}$ or larger.

Next, tlow results for different directions of flow were analyzed for equivalent porous medium behavior using the continuity test and the angle of flow test. Flow regions oriented at every $15^{\circ}$, beginning at orientation $0^{\circ}$, were used in this part of the study. Square flow regions of width $400 \mathrm{~cm}$ were used for orientations $0^{\circ}, 15^{\circ}, 30^{\circ}, 45^{\circ}, 60^{\circ}$, and $105^{\circ}$. Rectangular flow regions of size $186 \times 400 \mathrm{~cm}$ were used for orientations $75^{\circ}$ and $90^{\circ}$, because the angle of flow is greater than $45^{\circ}$ for these two orientations. Side 2 had to be longer than side 1 to ensure that a zone of continuous flow existed from side 2 to side 4.

A comparison of numerical and theoretical values for $q$ and $\theta$ is given in Table 5-1. The good ageement between numerical and theoretical values $\left(0.96<\mathrm{q} / \mathrm{q}_{\mathrm{T}}<1.04\right.$ and $\left|{ }_{\theta-\theta_{\mathrm{T}}}\right|<$ $3^{\circ}$ ) is evidence that the stability requirement for porous medium behavior has been satisied for all flow regions regardless of the orientation of the flow field. The angle of flow test is also satisfied for all orientations because the values computed for ANFC are essentially identical to those of ANFD. Furthermore, the continuity test is also satisfied because flow rates on c.pposing sides of the flow region aic equal. This equivalence results from the fact that an equal number of fractutes from each set intersect opposing sides of each flow region. Thus, each tlow region that has been tested exhibits porous medium flow behavior.

Directional equivalert porous medium flow behavior can be shown using the flow results in Table 5-1. Maicus and Evenson (1961) have derived very useful relationships between specific discharge and direction of flow for porous media. If the hydraulic gradient is kept con- 


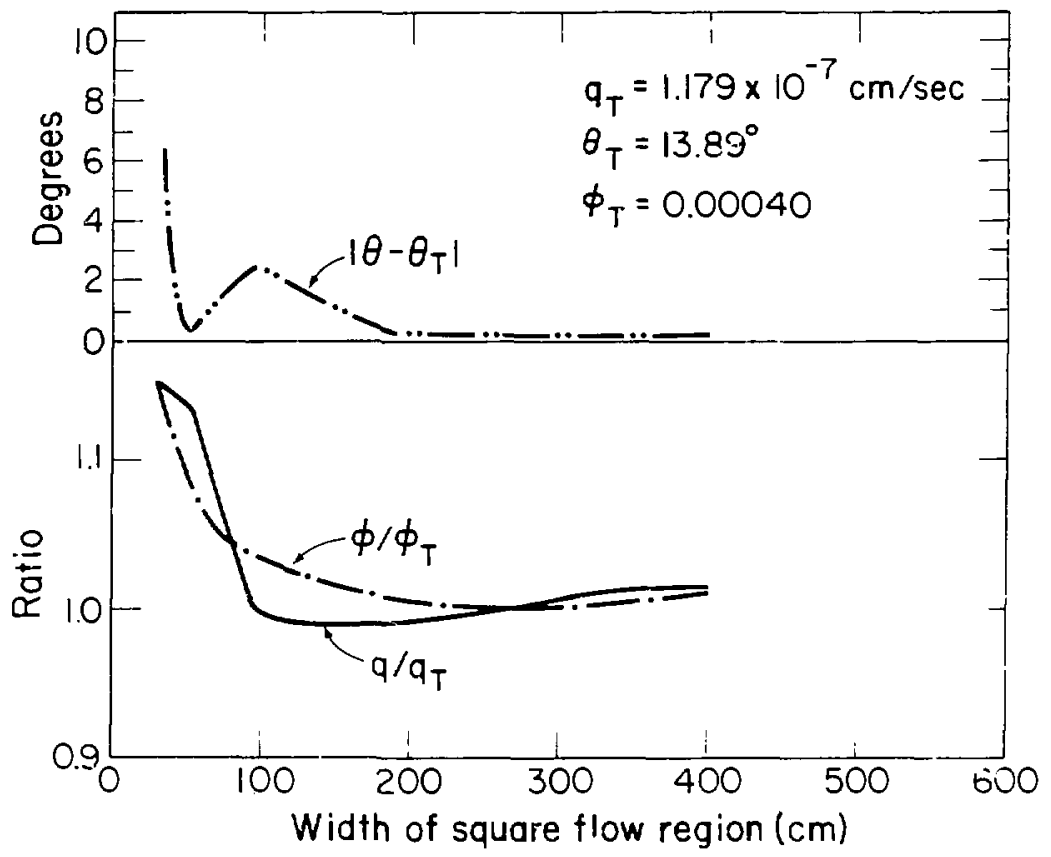

$X B\llcorner 833-1416$

Figure 5-2 Convergence of Specific Discharg: and Porosity to Their Theoretic $\lrcorner$ Values as Size of Flow Region increases (Rat. $s \mathrm{q} / \mathrm{q}_{\mathrm{T}}$ or $\phi / \phi_{\mathrm{T}}$ ). 


\begin{tabular}{|c|c|c|c|c|c|}
\hline \multirow{2}{*}{$\begin{array}{c}\text { Orientation } \\
\text { of Gradient } \\
\text { degrees }\end{array}$} & \multicolumn{2}{|c|}{ Specific Dischange } & \multicolumn{3}{|c|}{ Angle of Flow } \\
\hline & $\begin{array}{c}\text { Theoretical } \\
10^{-7} \mathrm{~cm}\end{array}$ & $\begin{array}{l}\text { Actual } \\
10^{-7} \frac{\mathrm{cm}}{\mathrm{s}}\end{array}$ & $\begin{array}{c}\text { Theoretical } \\
\text { degrees }\end{array}$ & $\begin{array}{l}\text { ANFC } \\
\text { degrees }\end{array}$ & $\begin{array}{l}\text { ANFD } \\
\text { degrees }\end{array}$ \\
\hline $\begin{array}{c}0 \\
15 \\
30 \\
45 \\
60 \\
75 \\
90 \\
105\end{array}$ & $\begin{array}{c}1179 \\
1.220 \\
1.179 \\
1.057 \\
0.8649 \\
0.6147 \\
0.3269 \\
0.08759\end{array}$ & $\begin{array}{c}1.199 \\
1.232 \\
1.184 \\
1.065 \\
0.8706 \\
0.6116 \\
0.3406 \\
0.0846\end{array}$ & $\begin{array}{c}13.90 \\
0 \\
-13.90 \\
-27.63 \\
-40.92 \\
-52.91 \\
-60.00 \\
0\end{array}$ & $\begin{array}{c} \\
13.65 \\
-1.47 \\
-13.84 \\
-26.86 \\
-40.82 \\
-52.76 \\
-61.32 \\
-2.87\end{array}$ & $\begin{array}{cc} & 13.65 \\
1 & 0 \\
1 & -13.84 \\
& -26.86 \\
& -40.82 \\
& -52.76 \\
& -61.32 \\
& -2.87\end{array}$ \\
\hline
\end{tabular}

stant. as was done in this study, the square root of specific discharge divided by $\cos \theta,(q / \cos \theta)^{1 / 2}$, when plotted versus flow direction, forms an ellipse since $q / \cos \theta$ is equal to the product of $K_{f}$ and the hydraulic gradient. Figure 5-3a shows the plot of $(q / \cos \theta)^{4 / 2}$ versus direction of flow. It may be seen that the sp ific discharge curve is an ellipse with directions of maximumı and minimum permeabilities near $15^{\circ}$ and $105^{\circ}$, respectively. The ellipse is symmetric about these two principal directions. As expected from theory, this particular network of continuous fractures has thic same flow behavior as an equivalent porous medium.

Having demonstrated that this system of continuous fractures behaves like an equivalent porous medium for fluid flow, the model was next used to investigate continuum behavior for transport. For comparative purposes, one needs the total porosity of the fractur'system. The porosity of each set is 0.0002 , which is simply the $0.002 \mathrm{~cm}$ aperture divided by the $10 \mathrm{~cm}$ spacing, and therefore the total porosity for the two sets is 0.0004 .

Figure 5-4 is a plot of the hydraulic effective porosity versus direction of flow, which was determired by adding the angle of flow to the orientation of the flow region. At orientation $90^{\circ}$ (whers the theoretical flow direction is $30^{\circ}$ ) and orientation $120^{\circ}$ (where the iheoretical flow direction is $\left.180^{\circ}\right)$ there is a dramiatic refuction in $\phi_{\mathbf{H}}$. Al either orientation, one set of fractures becomes nonconductiv a because it is perpendicular to the hydraulic gradient. The result is that $\phi_{H}=\phi / 2$ in either flow direction. The directional dependence in the hydraulic effectiv porosity shows that this fracture system does not behave like an equivalent porous medium for 


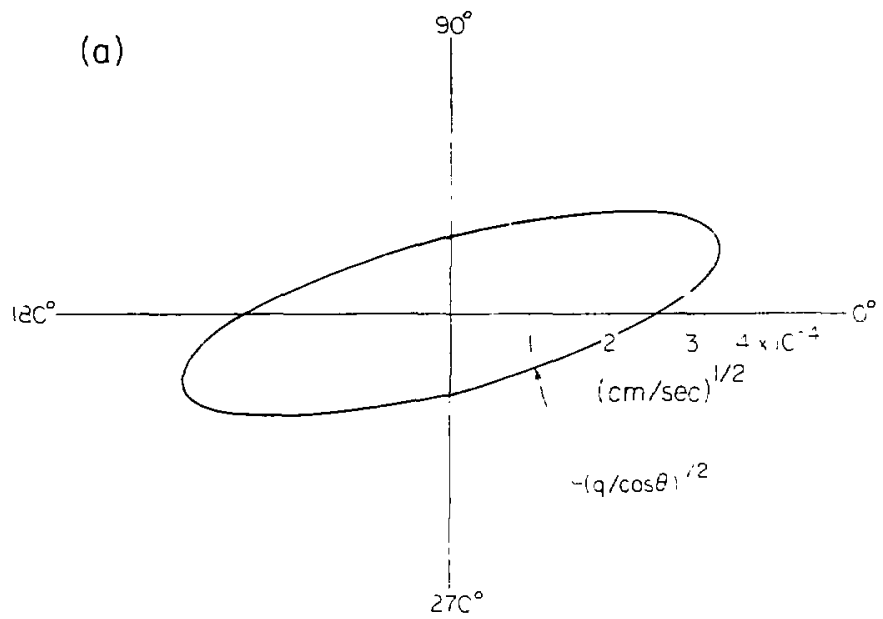

(b)

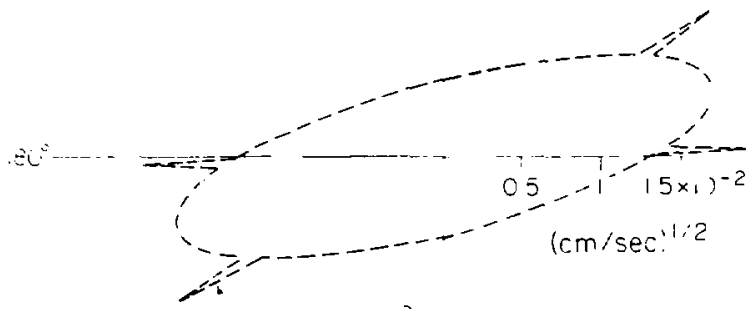

in $\sin 2 \sin 2$ 


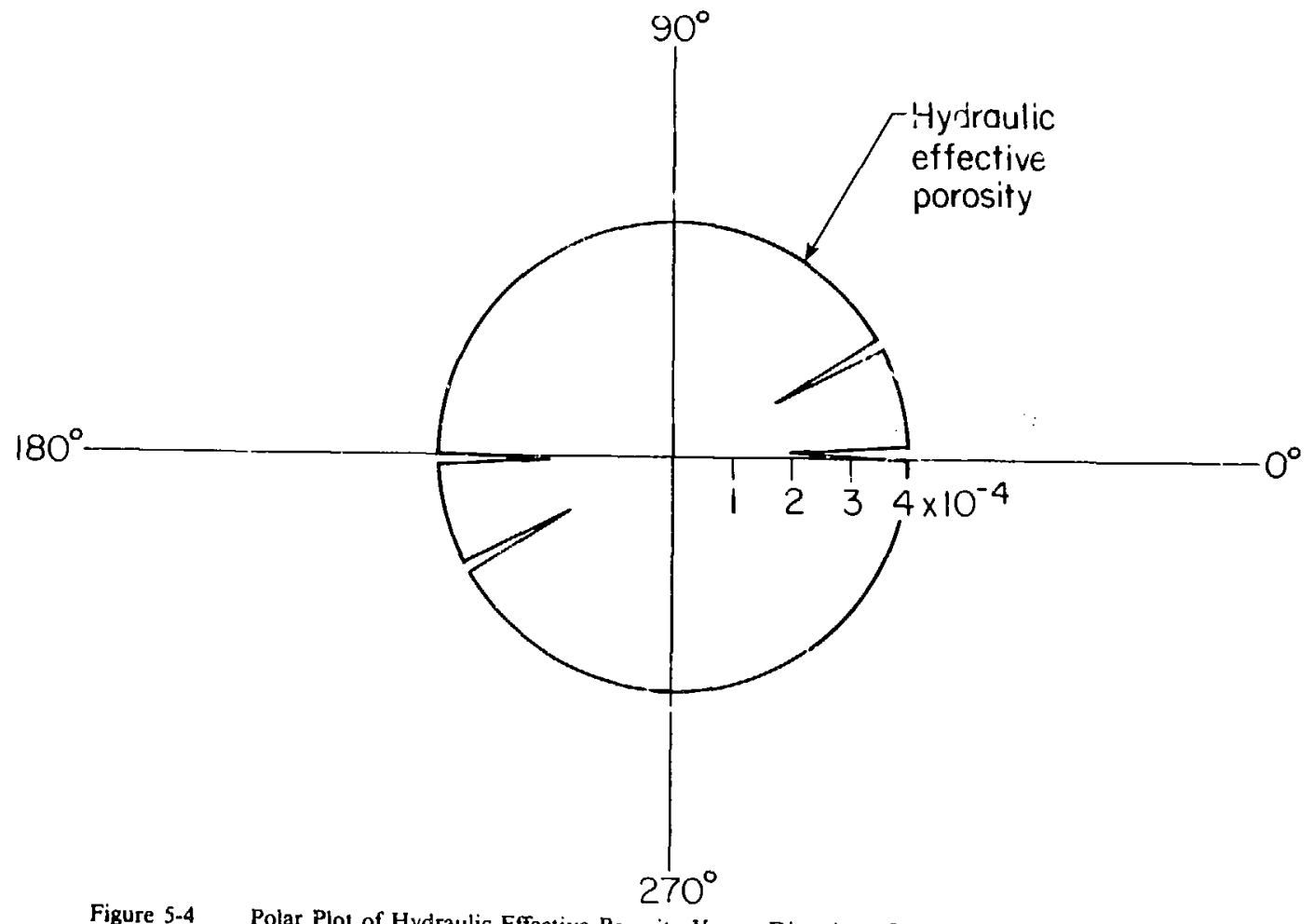
of Two Sers of Parallel. Continuous, and Constant Aperture Fractures. 
transport.

At orientation $105^{\circ}, \phi_{H}$ decreases slightly beluw $\phi$ and obtains a value of 0.000384 . To check this result, two additional square flow regions of widths 320 and $450 \mathrm{~cm}$ were tested. The resulting $\phi_{H}$ were 0.000385 and 0.000409 , respectivel; This clearly indicates that $\phi_{H}$ is converging to $\phi$ and that the size of the mechanical transport continuum varies with direction. However, the convergence proceeds at different rates for different directions.

The change in convergence rate is directly related to the tortuosity. As tortuos:ty increases, travel paths become more irtegular and deviate more from the flow direction. Consequently, larger flow regions are needed before representative mechanical transport behaviur occurs. Figure 5- $i$ shows that the tortuosity is relatively stable at 1.04 between directions $0^{\circ}$ to $30^{\circ}$. At direction $30^{\circ}$, the tortuosity reaches a theoretical minimum of 1.0 . Tortuosity then increases rapidly to 3.86 near direction $105^{\circ}$. The bisection of the hydraulic gradient with the obtuse angle of $150^{*}$ resulting trom the intersection of the two fracture sets (Figure 5-1) caused tortuosity to be maximum in this direction. Thus, the large tortuosity in this direction results in a slight oscillation in $\phi_{\mathrm{H}}$. Tortuosity should be inversely proportional to permeability for an equivalent porous medium. Since tortuosity is relatively stable between directions $0^{\circ}$ and $30^{\circ}$, tortuosity does not behave like it would for an equivalent porous mesium. However, between $30^{\circ}$ and $105^{\circ}$ tortuosity does behave like it would for an equivalent porous medium.

Figure 5-6 shows a plot of VPORE versus flow direction. This figure shows that there is no difference between VPORE and the mean pore velocity. In transport studies, tortuosity is rarely computed and normally assigned a value about $\sqrt{2}$. This figure also demonstrates that if a constant tortuosity of $\sqrt{\dot{\alpha}}$ had been used to compute VPORE. a serious error in the calculated mean pore velocity would have resulted.

If the hydraulic effective porosity is a constant, then the square root vi the average linear velocity divided by $\cos \theta$. (VLIN/ $\cos \theta)^{h}$, should plot as an ellipse for an equivalent porous medium since $(q / \cos \theta)^{\text {h }}$ would plot as an ellipse. The plot of $(V I I N / \cos \theta)^{m}$ in Figure 5-3b also demonstrates that this fracture system cannot be treated is equivalent porous medium for tran- 


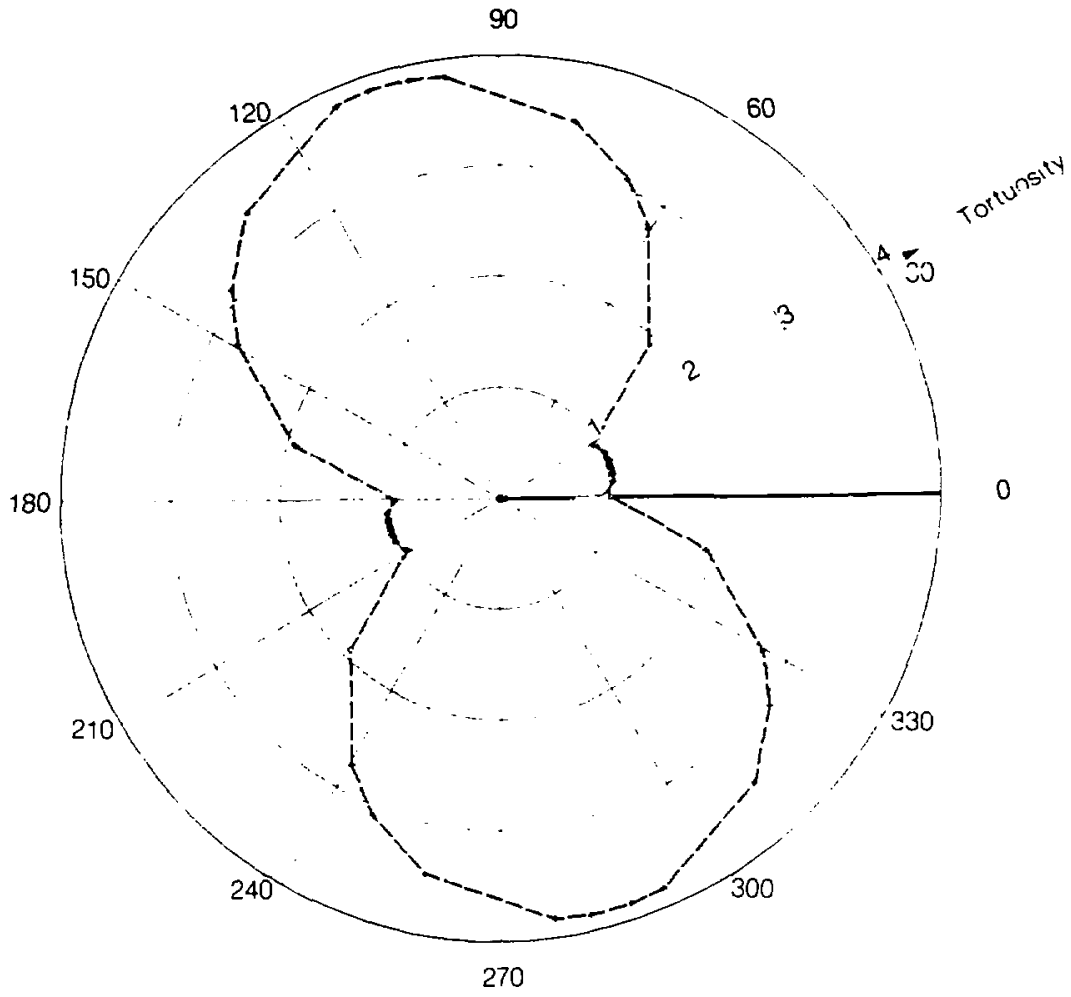

XBL 838-565

Figure 5-5 Tortuosity Versus Direction of :Tow for Fracture Systeni of Two Sets of Parallel, Contiruous, and Consten! Aperture Fractures. 


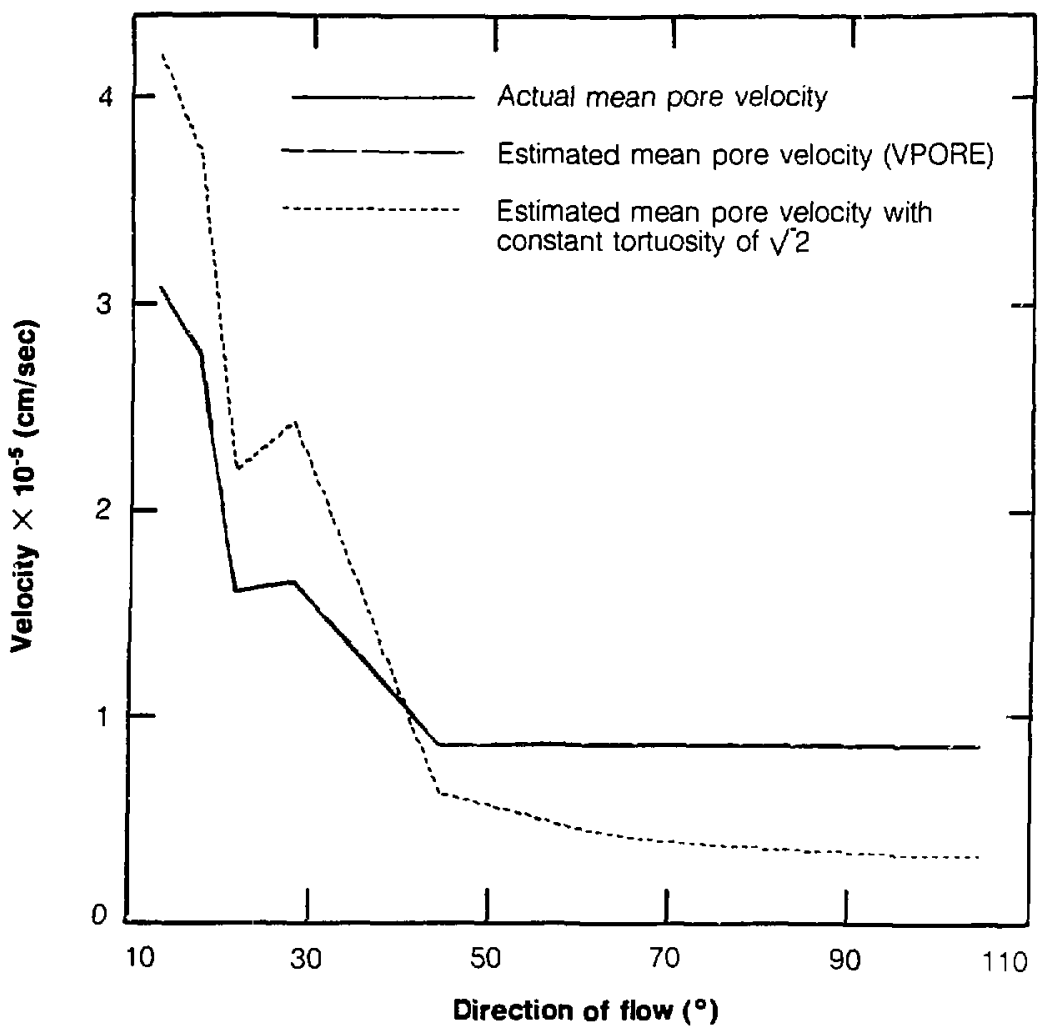

XBL 838-564

Figure S-6 Actual Mean Pore Velocity, Calculated Mean Pore Velocity, and Calculated Mean Pore Velocity With Tortuosity of $\sqrt{2}$ for Fracture System of Two Sets of Continuous, Parailel, and Constant Aperture Fractures. 
sport. Although the VLIN curve coincides with the q curve in most directions, there are four sharp cusps in the directions where $\phi_{\mathrm{H}}$ drops dramatically.

Figure 5-7 is the polar plot of the longitudinal geometric dispersivity. This plot definitely shows that $\alpha_{\mathrm{L}}$ is directionally dependent. The maximum $\alpha_{\mathrm{L}}$ of $74 \mathrm{~cm}$ occurs in the direction of minimum permeability. Longitudinal geometric dispersivity is much smaller in all other directions, but $\alpha_{\mathrm{L}}$ is zero only near directions of flow $30^{\circ}$ and $0^{\circ}$. Longitudinal geometric dispersivity is minimum in these directions because only one set conducts flow, and the velority in each fracture of this set is constant.

The applicability of the Fickian dispersive approach was investigated for flow direction $18.3^{\circ}$ (orientation $45^{\circ}$ ) using two square flow regions of widths 200 and $400 \mathrm{~cm}$. When the Fickian approach is applicable, $\alpha_{\mathrm{L}}$ will not vary with the size of the flow region. The longitudinal geometric dispersivities computed for the two sizes were $0.882 \mathrm{~cm}$ and $0.995 \mathrm{~cm}$, respectively. The scale-dependent dispersivity shows that the Fickian approach cannot be applied at this scaie.

\subsection{SYSTEM WITH TWO ORTHOGONAL SETS OF CONTINUOUS FRACTURES}

In the second investigation with this model, the system consisted of two sets of parallel fractures oriented at righ! angles to each other and all spaced $10 \mathrm{~cm}$ apart as shown in Figure 5-8. Anisotropy was achieved by using an aperture of $0.002 \mathrm{~cm}$ for the firsi set oriented at $0^{\circ}$, and an aperture of $0.004 \mathrm{~cm}$ for the second set oriented at $90^{\circ}$. Thus, the direction of maximum principal permeability is $90^{\circ}$, and the direction of minimum principal permeabiliiy is $0^{\circ}$. The hydraulic gradient along sides 1 and 3 (see Figure 3-4) was set at 0.01 for all flow regions. The total porosity is 0.0006 ; the porosity for the set oriented at $0^{\circ}$ is 0.0002 and that for the set at $90^{\circ}$ is 0.0004 .

Sizes of flow regions were selected so that the number of elements and nodes in each region was nearly equal to that of the first study. It was anticipated that using the same number of elements and nodes would produce equivalent porous medium flow behavior in this orihogonal fracture system. Square flow regions of width $280 \mathrm{~cm}$ were used for orientations $0^{\circ}$, 


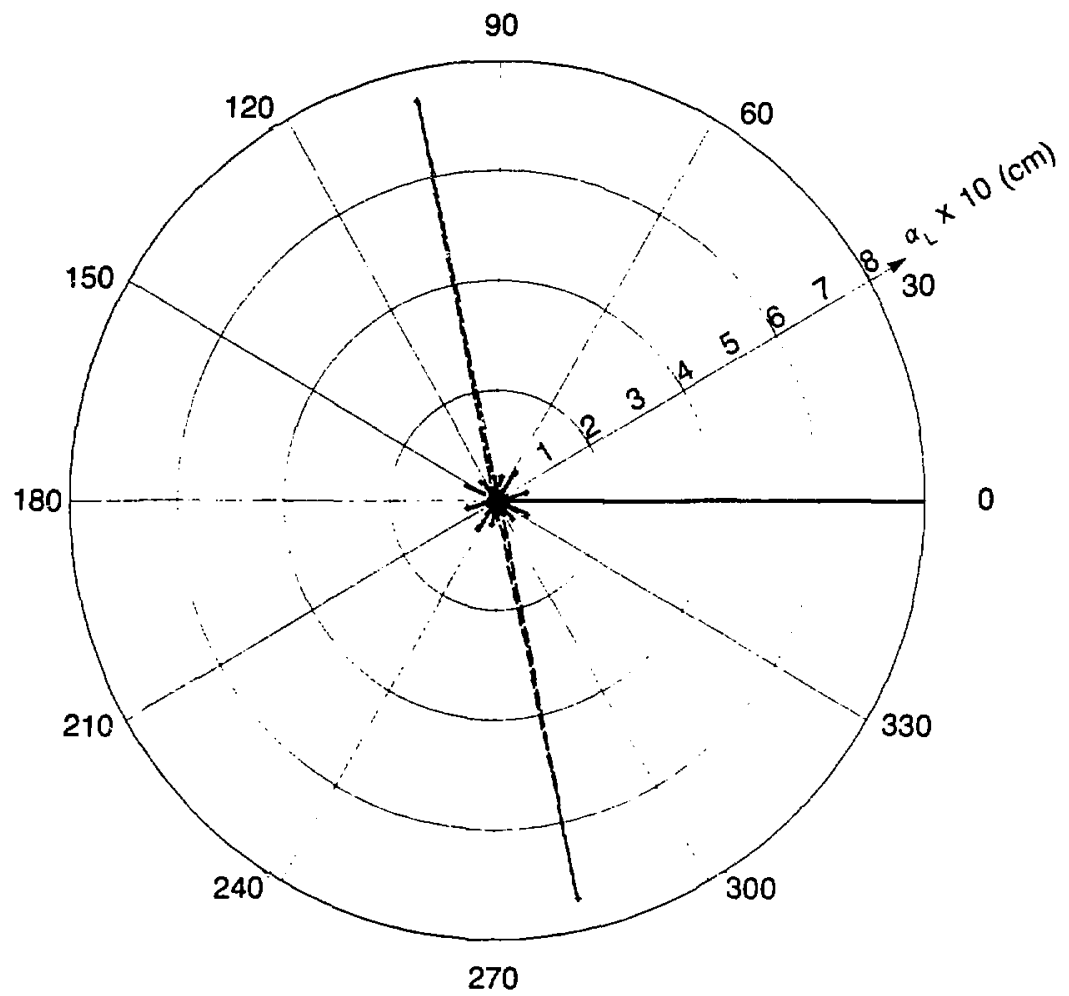

XBL $841-387$

Figure 5-7 Polar Ploî of Longitudinal Geometric Dispersivity for Fracture System of Two Sets of Continuous, Parallel, and Constant Aperture Fractures. 


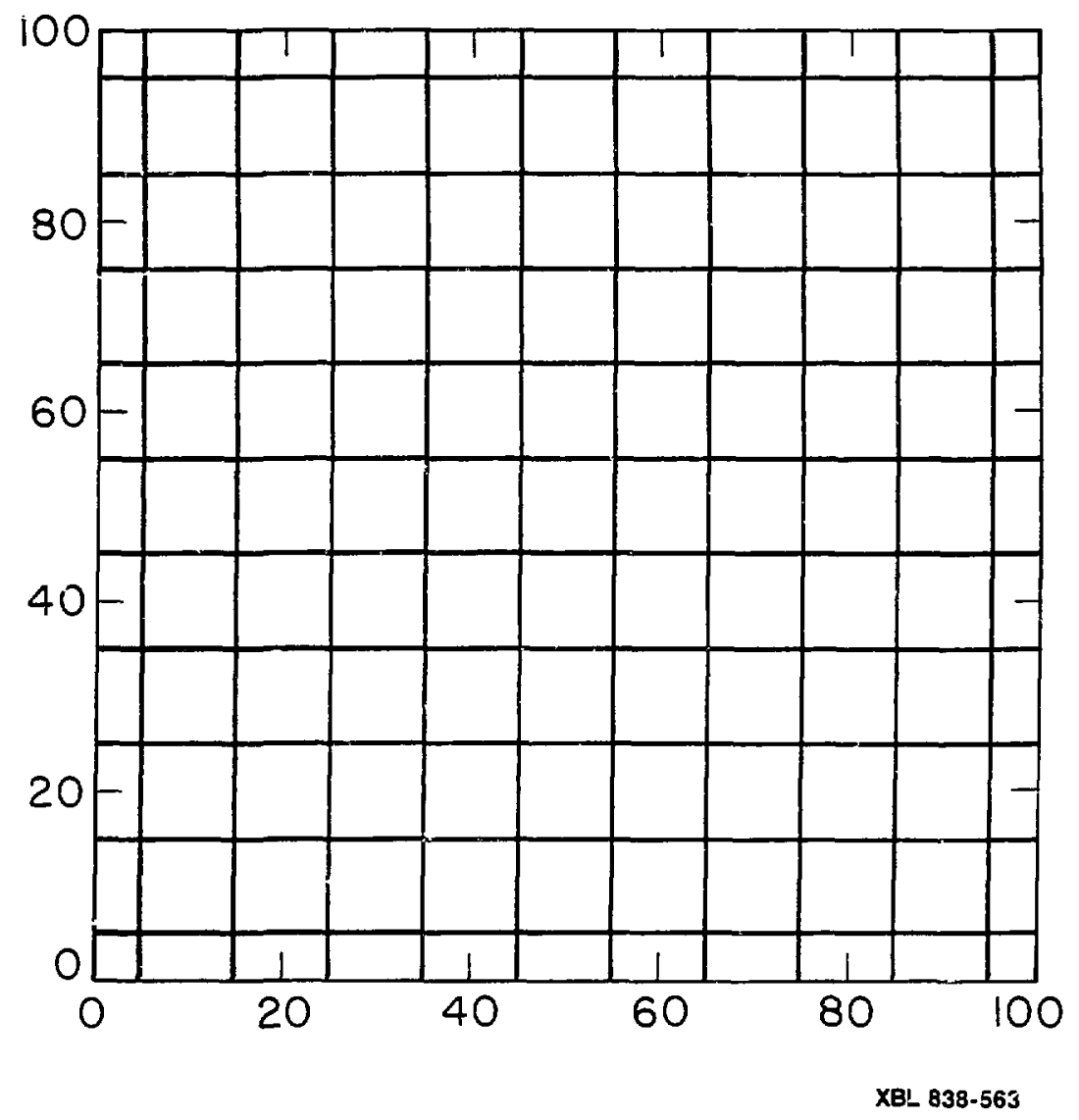

Figure 5-8 Fracture System With Two Orhogonal Sets of Continuous Fractures. 
$45^{\circ}, 60^{\circ}, 75^{\circ}$ and $90^{\circ}$. Rectangular flow regions of $235 \times 335 \mathrm{~cm}$ were used for orientations $15^{\circ}$ and $30^{\circ}$ because the angle of flow was greater then $45^{\circ}$.

A comparison of numerical and theoretical values for specific discharge is given in Table 5-2. The results demonstrate that each flow region behaves like an equivalent porous medium for fluid flow. Figure 5-9a illustrates directional equivalent porous medium behavior. The plot of $(q / \cos \theta)^{h}$ is an ellipse whose maximum axis coincides with the maximum principal permeability at $90^{\circ}$ and whose minimum axis coincides with the minimum value of permeability at $0^{\circ}$. This is further evidence that this system of orthogonal fractures behaves like an equivalent porous medium for fluid flow.

Table 5-2. Specific Discharge Results for Fracture System With Two Orthoponal Sets of Fractures.

\begin{tabular}{|c|c|c|c|c|c|c|c|}
\hline \multirow{2}{*}{$\begin{array}{c}\text { Orientation } \\
\text { of Gradient } \\
\text { degrees }\end{array}$} & \multicolumn{2}{|c|}{ Specific Dischange } & & \multicolumn{4}{|c|}{ Angle of Flow } \\
\hline & $\begin{array}{l}\text { Theoretical } \\
10^{-7} \frac{\mathrm{cm}}{\mathrm{s}}\end{array}$ & $\begin{array}{l}\text { Actual } \\
10^{-7} \frac{\mathrm{cm}}{\mathrm{s}}\end{array}$ & & $\begin{array}{c}\text { Theoretical } \\
\text { degrees }\end{array}$ & $\begin{array}{l}\text { ANFC } \\
\text { degrees }\end{array}$ & & $\begin{array}{l}\text { ANFD } \\
\text { degrees }\end{array}$ \\
\hline $\begin{array}{c}0 \\
15 \\
30 \\
45 \\
60 \\
75 \\
90\end{array}$ & $\begin{array}{l}0.6538 \\
1.496 \\
2.676 \\
3.727 \\
4.541 \\
5.055 \\
5.230\end{array}$ & $\begin{array}{c}0.6538 \\
1.499 \\
2.691 \\
3.681 \\
4.668 \\
5.036 \\
5.230\end{array}$ & | & $\begin{array}{c}0 \\
49.99 \\
47.78 \\
37.87 \\
25.87 \\
13.08 \\
0\end{array}$ & $\begin{array}{c}-0.31 \\
50.91 \\
47.52 \\
38.08 \\
25.64 \\
14.72 \\
0\end{array}$ & $\vdots$ & $\begin{array}{c}-0.40 \\
50.90 \\
46.82 \\
37.87 \\
25.12 \\
i 4.59 \\
0\end{array}$ \\
\hline
\end{tabular}

The model was next used to investigate continuum behavior for transport, and the results are shown in Figure 5-10. We again see a drastic reduction in hydraulic effective porosity when the direction of gradient is at right angles to either fracture set. Figure 5-10 clearly illustrates the directional dependence of hydraulic effective porosity for this orthogonal fracture system. The plot of $(\text { VLIN } / \cos \theta)^{\text {sh }}$ in Figure $5-9 \mathrm{~b}$ reveals an unexpected result. One would normally associate the direction of minimum principal permeability as an indication of the direction of the minimum velocity. However, the minimum value of VLIN does not occur at $0^{\circ}$ because the minimum hydraulic effective porosity occurs in this direction. In dealing with fracture networks of this 'ind, one simply can.ot associate directions of principal permeabilities with the directions of maximum or minimum linear velocities. 

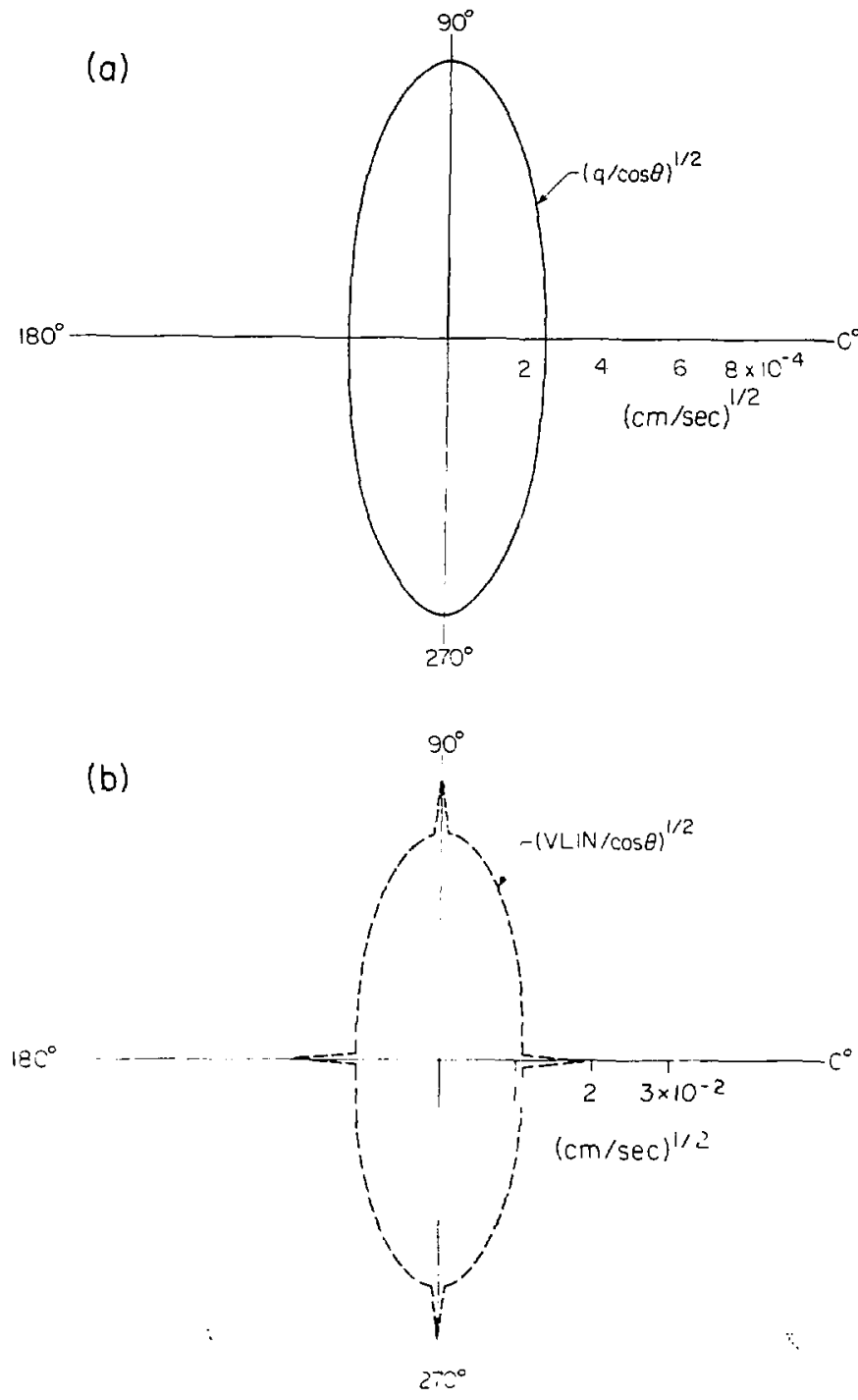

Figure 5-9 Polar Plots of a) Specific Discharge and b) Average Linear Factors Versus Direction of Flow for System With Two Orthogonal Sets of Continuous Fractures. 


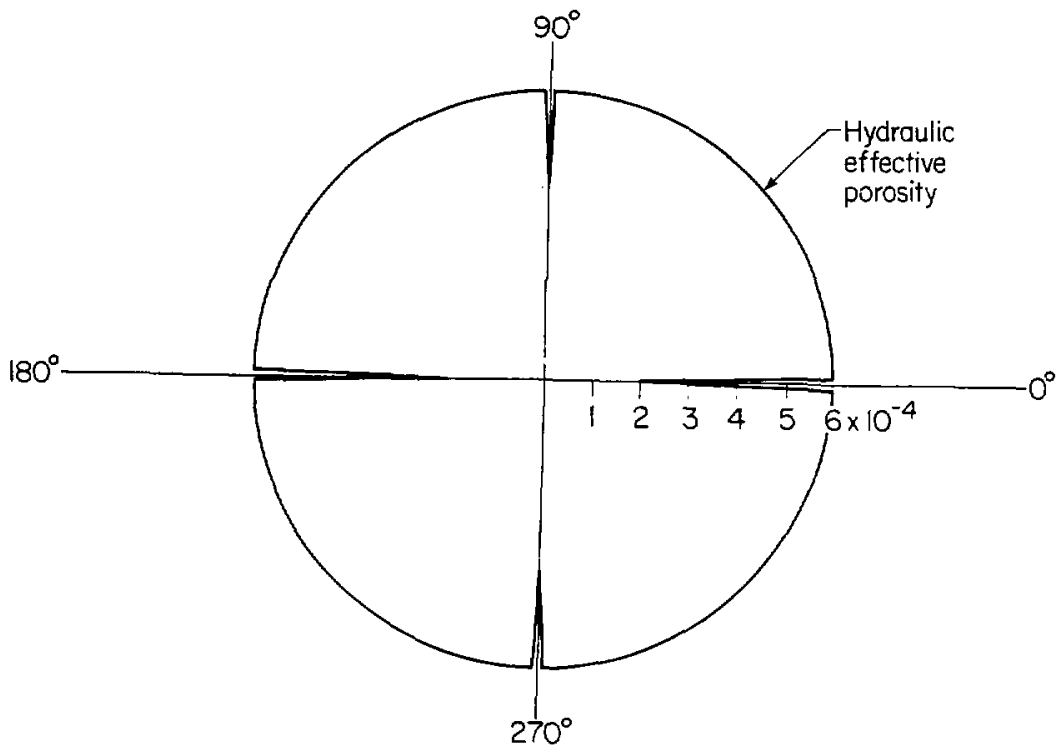

XBL B33-1422

Figure 5-10 Polar Plot of Sydrauiic Effective Porosity for System of Two Orthogonal Sets of Continuous Fractures. 
The tortuosity versus direction of flow is shown in Figure 5-11. The directional nature in tortuosity shows that this parameter does not behave like it would for an equivalent porous medium. This is clearly evident in the direction of minimum permeability where tortuosity is minimum. This is exactly opposite of what one would expect for an equivalent porous medium.

The polar plot in Figure 5-12 shows that $\alpha_{L}$ is strongly directionally dependent. The maximum $\alpha_{L}$ of $26 \mathrm{~cm}$ is obtained in the four directions $7^{\circ}$ from the direction of maximum permeability. In the two principal directions, $\alpha_{\mathrm{L}}$ is zero because only one of the sets conducts flow, and the velocity in each fracture for this set is constant. Thus unlike the previous continuous system where $\alpha_{\mathrm{L}}$ reached its maximum value in the direction of minimum permeability, $\alpha_{\mathrm{L}}$ has a minimum value in the direction of minimum permeability.

The polar plots of $\alpha_{\mathrm{L}}$ for the two continuous fracture systems both show large directional variations in $\alpha_{\mathrm{L}}$. The maximum $\alpha_{\mathrm{L}}$ is much larger in the first continuous system where anisotropy is greater. If a directionally-stable $\alpha_{\mathrm{L}}$ is used to model transport for each system, serious errors in transport prediction would result. Yet, this type of modeling is presently practiced by treating an anisotropic medium as an equivalent isotropic medium.

This orthogonal fracture system can easily be made isotropic by making the apertures for both sets ihe same. Theoretically, $\alpha_{\mathbf{L}}$ is directionally stable for an isotropic porous medium. If this fracture system were converted to an isotropic medium, a directionally stable $\alpha_{\mathrm{L}}$ would not result. In directions of flow $0^{\circ}$ and $90^{\circ}$, longitudinal geometric dispersivity would be zero. In all other directions, longitudinal geometric dispersivity would be nonzero. This clearly shows that a system which behaves like an equivalent isotropic porous medium for fluid flow, may not have a $\alpha_{1}$ which is directionally stable as theoretically expected. 


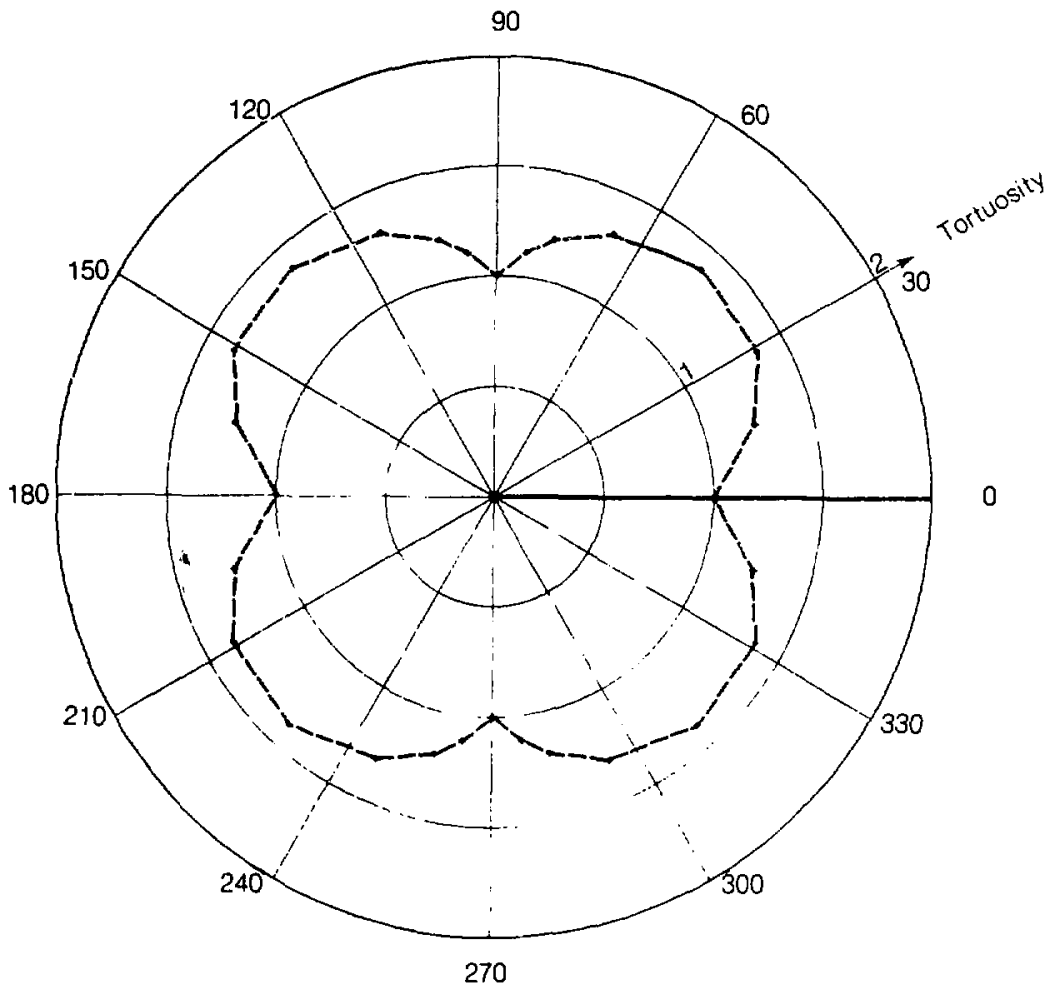

XBL 838-562

Figure 5-11 Polar Plot of Tortuosity for Fracture System With Two Orthogonal Sets of Continuous Fractures. 


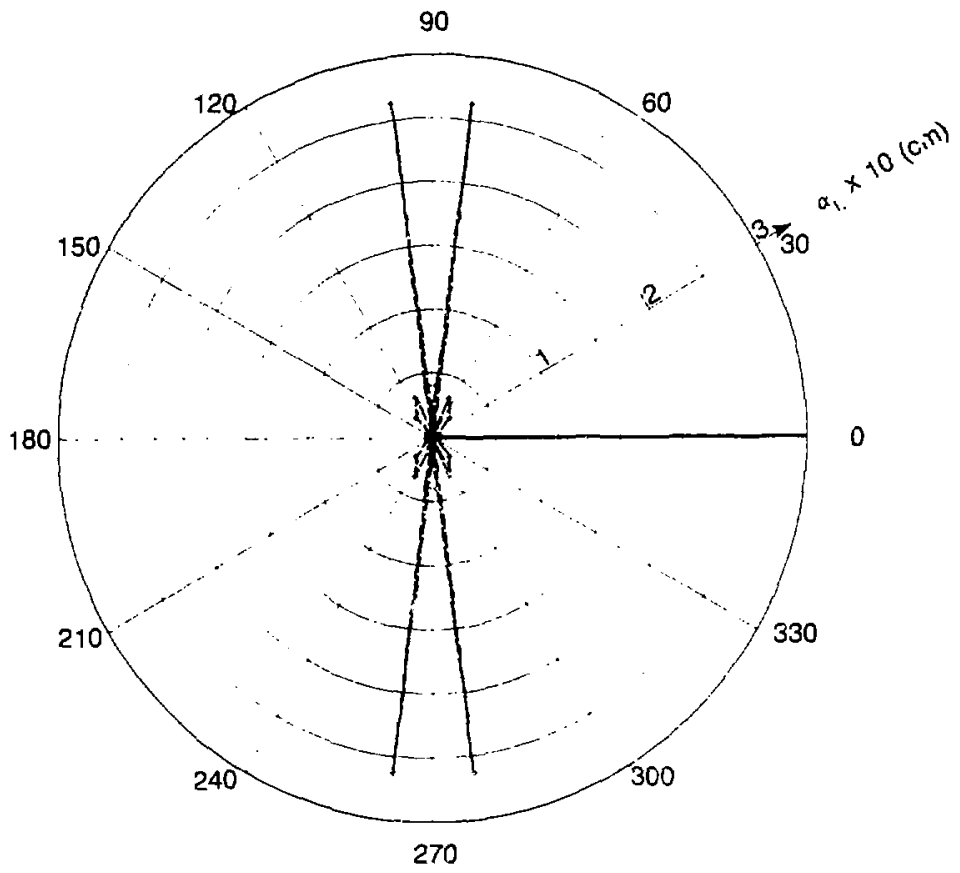

$X B i \quad 841-388$

Figure 5-12 Polar Plot of Longitudinal Geometric Dispersivity for System With Two Orhogonal Sets of Continuous Fractures. 


\section{CHAPTER 6 \\ INVESTIGATION OF DISCONTINUOUS \\ FRACTURE SYSTEMS}

\subsection{INTRODUCTION}

A continuous fracture system is created in the generation region when all fractures are long compared to the size of the generation region. However, it more likely that a fracture system must be studicd in which the fractures do not span the width of the region. A fracture system consisting of finite-length fractures is called a discontinuous fracture system. Discontinuous fracture systems are much more difficult to analyze than continuous fra'-ture systems. Continuous fracture systems have inen shown to behave like equivalent porous media for fiuid flow. This is not the case for discontinuous systems. The concepts developed in section 3.9 are used to evaluate equivalent porous medium flow behavior for discontinuous systems.

Mechanical transpon is influenced by the paths of fluid flow in the conductive void spaces of a fracture system. Flow paths differ in discontiauous and continuous systems due to the structure of the void regions. In a continuous fiac sre system, all thactures are connected such that fluid can flow through the entire void region. However, conductive spaces are only par of the total void region in a discontinuous fracture system. The void region also consists of dead-end zones and isolated spaces where fluids cannot flow.

\subsection{DISCONTINUOUS FRACTURE SYSTEM OF TWO SETS ORIENTED AT $0^{\circ}$ AND $30^{\circ}$}

The first discontinuous fracture system studied was chosen to simulate the continuous fracture system in section 5.2. The discontinuous system consisted of two sets of fractures oriented at $0^{\circ}$ and $30^{*}$. The areal density for each set was $0.00633 \mathrm{~cm}^{-2}$. All fractures had ar. aperure of $0.002 \mathrm{~cm}$ and a length of $40 \mathrm{~cm}$. A Monte Cario simulation vas required because fracture centers were randomly located in the generation region. The Monte Carlo simulation consisted of 10 realizations; the size of generation region used in each realization was $303 \times 300$ 
cm. Figure 6-1 shows the fracture pattern in the generation,$n$ for one of the realizations. The fracture pattern for the discontinuous fracture system differed considerably from the simulated continuous fracture system because of the random location of fracture centers and finite length of fractures.

The objective for each realization was to obtain a representative directional sample of mechanical transport and fluid flow properties. This required estimating the direction of flow for a given orientation of the hydraulic gradient. Since the angle of flow cannot be computed from first principles for discontinuous systems. the direction of flow for a given orientation of the hydraulic gradient was estimated from $\theta$ calculated for the simulated continuous fracture system. Based on these calculations, nine orientations of a uniform hydraulic gradient of $0 . n 1$ were selected to study fluid flow and mechanica! transport. The nine orientations and estimated directions of flow are listed in Table 6-1.

The flow region sizes used in the Monte Carlo simulation were determined from a size study conducted in the first realization for orientation $15^{\circ}$. In the size study, the width of a square flow region oriented at $15^{\circ}$ was slowly increased until the flow field exhibited the characteristics of an equivalent porous medium. This meant that the following conoitions had to be satisfied: continuity test, angle of flow test, and stability $c \vec{l} \vec{q}$. When the width of the flow region was $180 \mathrm{~cm}$, DEVF equiled 1.21 , DEVA equaled $1.84^{\circ}$, and $\mathrm{q}$ was relatively stable. Consequently, a minimum flow region size of $32400 \mathrm{~cm}^{2}$ was used in the Monte Carlo simulation. Tanle 6-1 ists the flow region sizes used to initiate the Monte Carlo simulation. The actual dimensions of the flow rejions were selected such thai a zone of continuous flow existed between sides 2 and 4 for the estimated direction of flow. Figure $6-2$ shows the fracture vattern and connected fracture segments in a flow region oriented at $83^{\circ}$ in one of the rea'izations.

Figure 6-3 is a polar plot of the mean square root of permeaibility in the direction of flow $\sqrt{K_{f}}$. Mean $\sqrt{K_{f}}$ was computed by averaging $\sqrt{K_{r}}$ for the ten realizations. For each mean $\sqrt{\mathrm{K}_{f}}$, the standard error of the mean was computed. The standard enor of i'se mean is a measure of the scatter in the data and is defined as the sampie standard deviation divided by the 


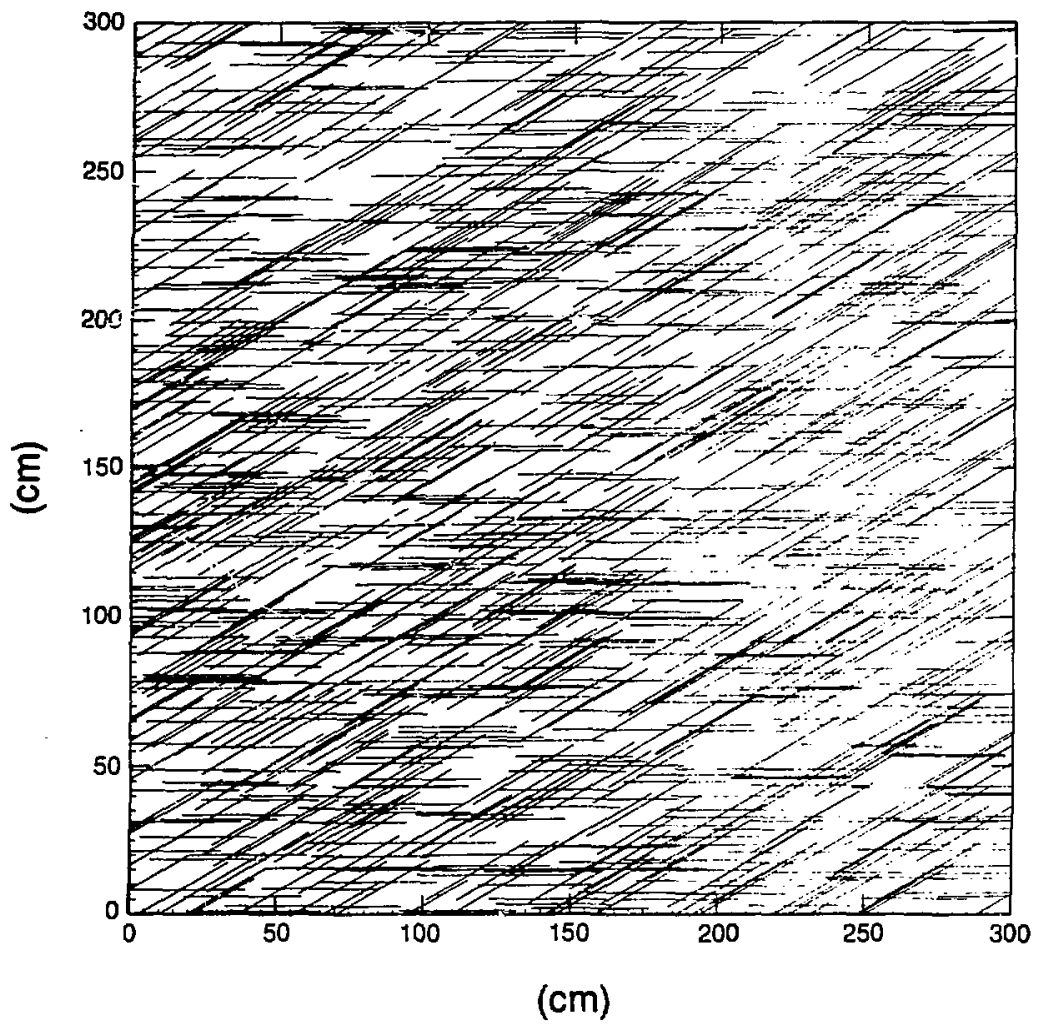

XBL $441-389$

Figure 6-1 Fracture Network in the Generation Region for Discontinuous Fracture System of Two Sets of Fractures Oriented at $0^{\circ}$ and $30^{\circ}$ With Constant Aperture and Length. 

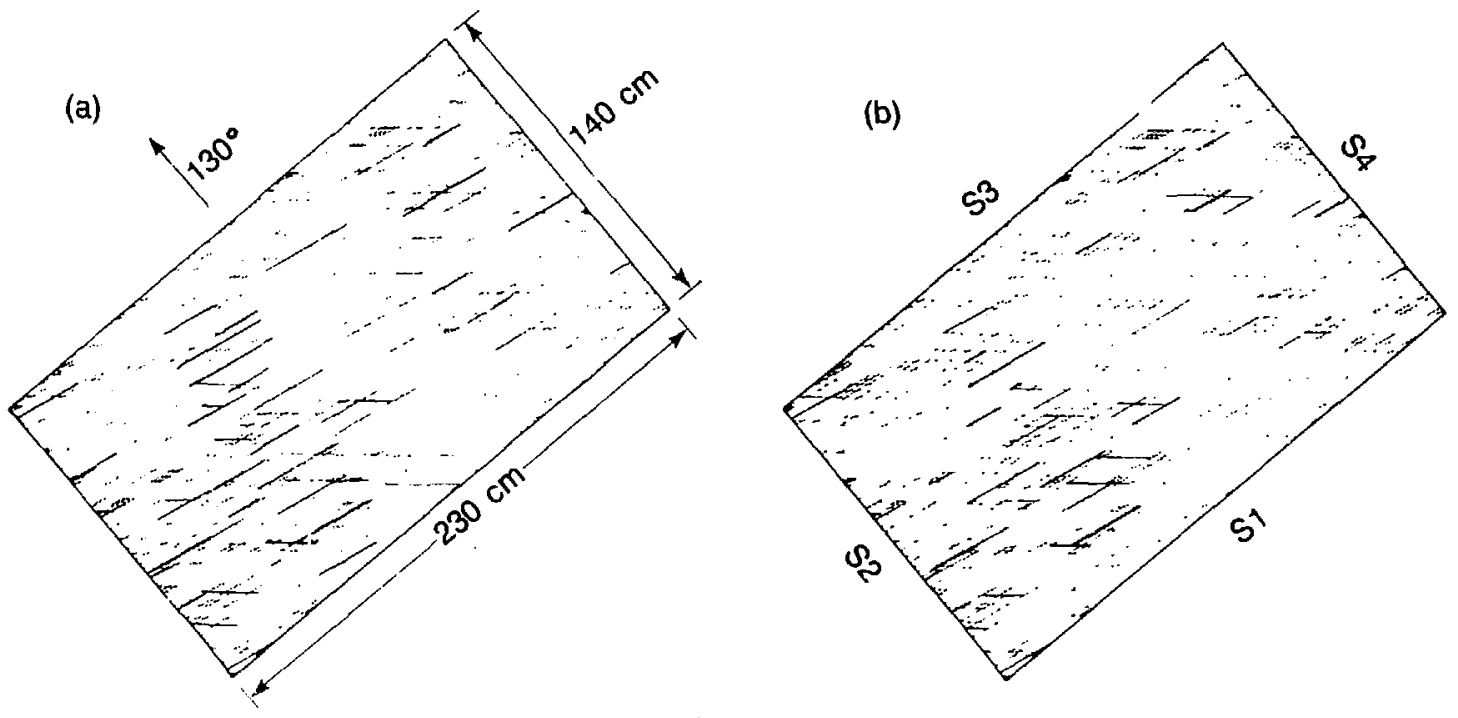

Figure 6-2 Networks of a) Fractures and b) Connected Fracture Segments in Fow Region Oriented at $130^{\circ}$ for Discontinuous Fracture System of Two Sets Oriented at $0^{\circ}$ and $30^{\circ}$. 


\begin{tabular}{|c|c|c|}
\hline $\begin{array}{l}\text { Orientation } \\
\text { of hydraulic } \\
\text { gradient } \\
\text { degrees } \\
\end{array}$ & $\begin{array}{c}\text { Direction of flow } \\
\text { based on continuous } \\
\text { system in section } 5.2 \\
\text { degrees }\end{array}$ & $\begin{array}{c}\text { Flow region size } \\
\mathrm{cm}^{2} \\
\end{array}$ \\
\hline $\begin{array}{c}15 \\
83 \\
100 \\
104 \\
105 \\
106.5 \\
110 \\
130 \\
160\end{array}$ & $\begin{array}{c}15 \\
25.08 \\
54.37 \\
91.34 \\
105 \\
125 \\
155.6 \\
186.2 \\
192.1\end{array}$ & $\begin{array}{l}180 \times 180 \\
140 \times 230 \\
170 \times 188 \\
180 \times 180 \\
180 \times 180 \\
170 \times 188 \\
170 \times 188 \\
140 \times 230 \\
180 \times 180\end{array}$ \\
\hline
\end{tabular}

square root of the number of realizations (Topping, 1955; Baird, 1962). The computed mean from the data has a probability of approximately 68 percent of being within \pm one standard error of the true value. The standard error of the mean $\sqrt{K_{f}}$ was less than $0.00006(\mathrm{~cm} / \mathrm{s})^{1 / 2}$ in all directions except in mean direction of flow $15.14^{\circ}$ where the stanciard error was 0.00010 $(\mathrm{cm} / \mathrm{s})^{1 / 2}$. In this direction, there is an irregularity in the $\sqrt{\mathrm{K}_{\mathrm{f}}}$ curve. The plot of mean $\sqrt{\mathrm{K}_{\mathrm{f}}}$ is similar to the plot of $(q / \cos \theta)^{4}$ for the simulated continuous iracture system (Figure 5-3). The shape of the mean $\sqrt{K_{f}}$ curve is approximately an ellipse with directions of maximum and minimum fermeabilities near $15^{\circ}$ and $105^{\circ}$, respectively. The ratio of $K_{x}$ to $K_{y}$ is about eleven. Thus, the directional flow characteristics for this fracture system behave like an equiralent porous medium.

Equivalent porous medium flow behavior was also e 'raluated for each direction of flow using the parameters DEVA and DEVF. When DEVA and DEVF are both small. equivalent porous medium behavior is likely to occur in that particular direction. Mean DEVA and mears DEVF are plotted versus direction of how in Figuse 6-4. DEVA exhibits two local maxima near each principal direction. The general tendency of the DEVA curve is for this parameter to increase as direction increases from the direction of maximum permeability to the drection of minimum permeability. DEVF also increases as direction of flow moves from the direction of maximum permeability to the direction of minimum permeab.lity. Thus. porous medium 


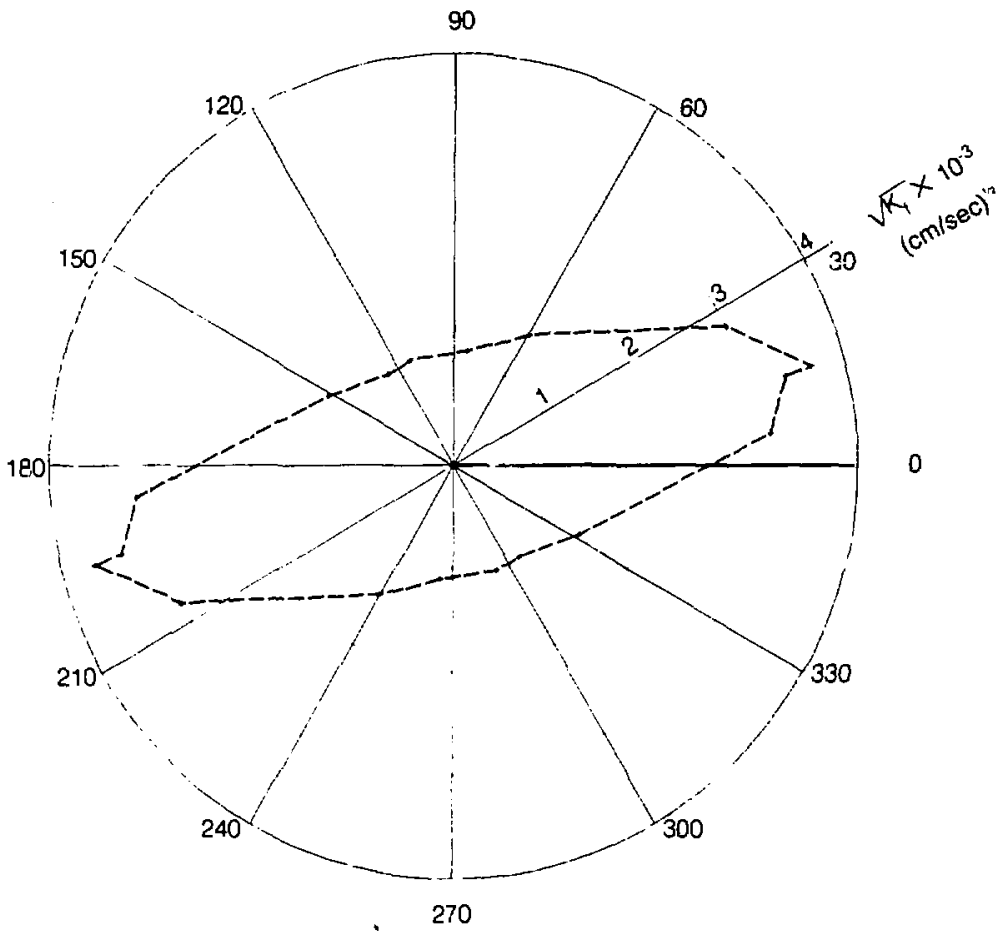

XEL 939-87T

Figure 6-3 Polar Plot of Square Root of Permeability in Direction of Flow for Discontinuous Fracture System of Two Sets Oriented at $0^{\circ}$ and $30^{\circ}$. 


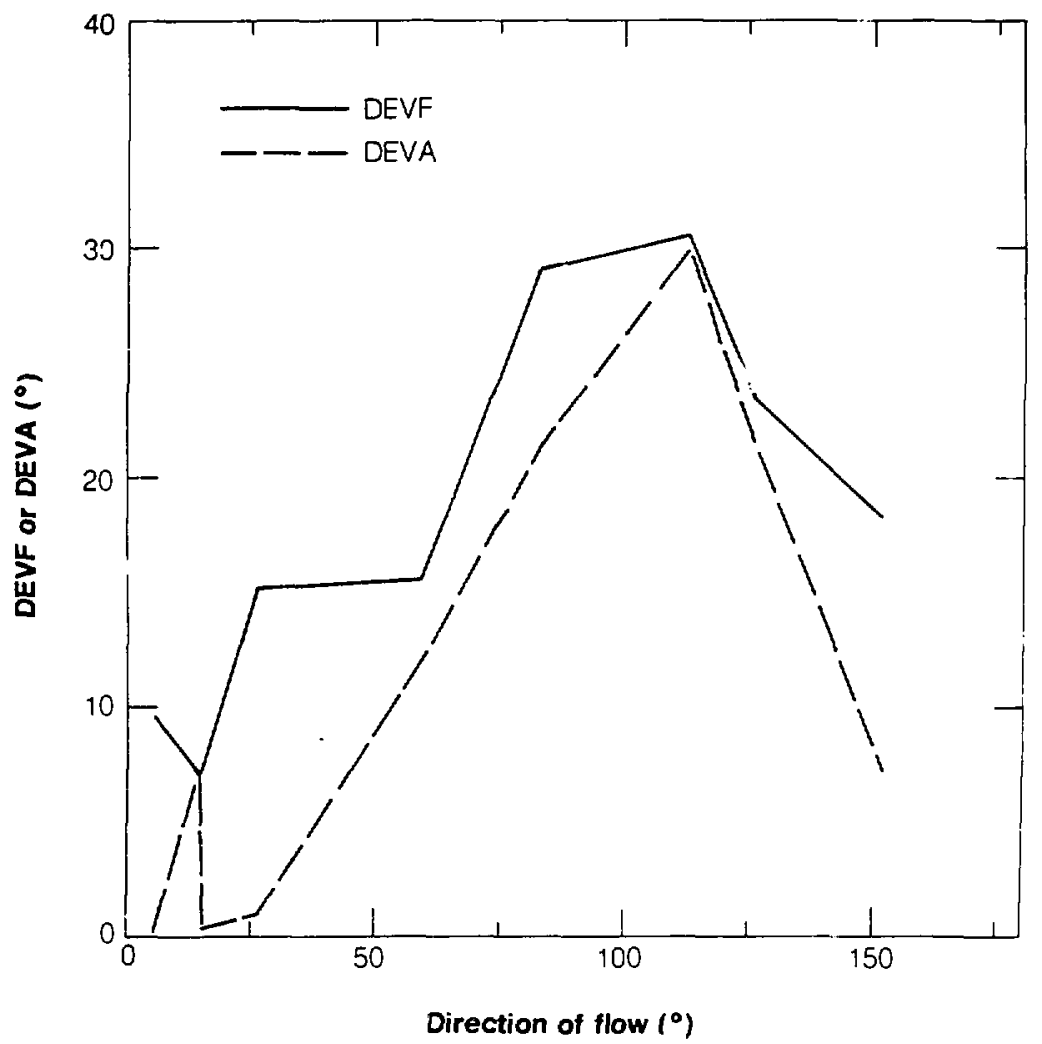

$\times B L 839-873$ Fracture System of Two Sets Oriented at $0^{\circ}$ and $30^{\circ}$. 
equivalence is more likely to occur near the direction of maximum permeability than near the direction of minimum permeability.

Figure 6-5 demonstrates that tortuosity is highly directionally dependent ranging from 1.10 to 3.93. The standard error of the mean $r$ was less than 4 percent of its mean value in any direction. The directional variation of tortuosity for this system is similar to tortuosity for the simulated continuous fracture system (Figure 5-5). However, there is one important difference. The minimum tortuosity occurs near the direction of maximum permeability and not at $30^{\circ}$, as found in the continuous system, such that tortuosity increases from the direction of maximum permeability to the direction of minimum permeability. Consequently, directional tortuosity for this system exhibits the characteristics one would expect for an equivalent porous medium. Tortuosity is used in this study to compute VPORE. Figure 6-6 shows now VPORE and MPV vary with direction of flow in the first realization. VPORE does not correspond exactly with MPV as found for the continuous fracture ijstem. However, VPORE provides a good estimate of MPV from two mechanical transport parameters $\mathrm{\tau}$ and VLIN. If tortuosity had been ignored in computing VPORE, a much lower estimate of the mean pore velocity would have resulted near the direction of minimum permeability.

The mean total porosity, rock effective porosity and hydrauli: effective porosity are each plotted asainst direction of flow in Figure 6-7. Total porosity and rock effective porosity are both directionally stable. Hydraulic effective porosity exhibits no sharp cusps as found in $\phi_{\boldsymbol{H}}$ for the simulated continuous fracture system, but there is some directional dependence. The minimum $\phi_{H}$ occurs near the direction of maximum permeability and the maximum $\phi_{H}$ occurs near the direction of minimum permeability. The mean $\phi_{\mathbf{H}}$ is nearly equal to the average of $\phi$ and $\phi_{R}$. Hydraulic effective porosity is computed as the product of $q$ and $\vec{t}$, divided by $L$. So $\Phi_{H}$ can be large when $\bar{\tau}$ is large. In the direction of maximum permeability, mean travel time is small because this i. the direction in which fluid flows the easiest. However, in the direction of minimum permeability, zones of low velocity and slow movement exist in the void region. Consequently, $\bar{\tau}$ and $\dot{\varphi}_{H}$ are large in the direction of minimum permeability. However, the 


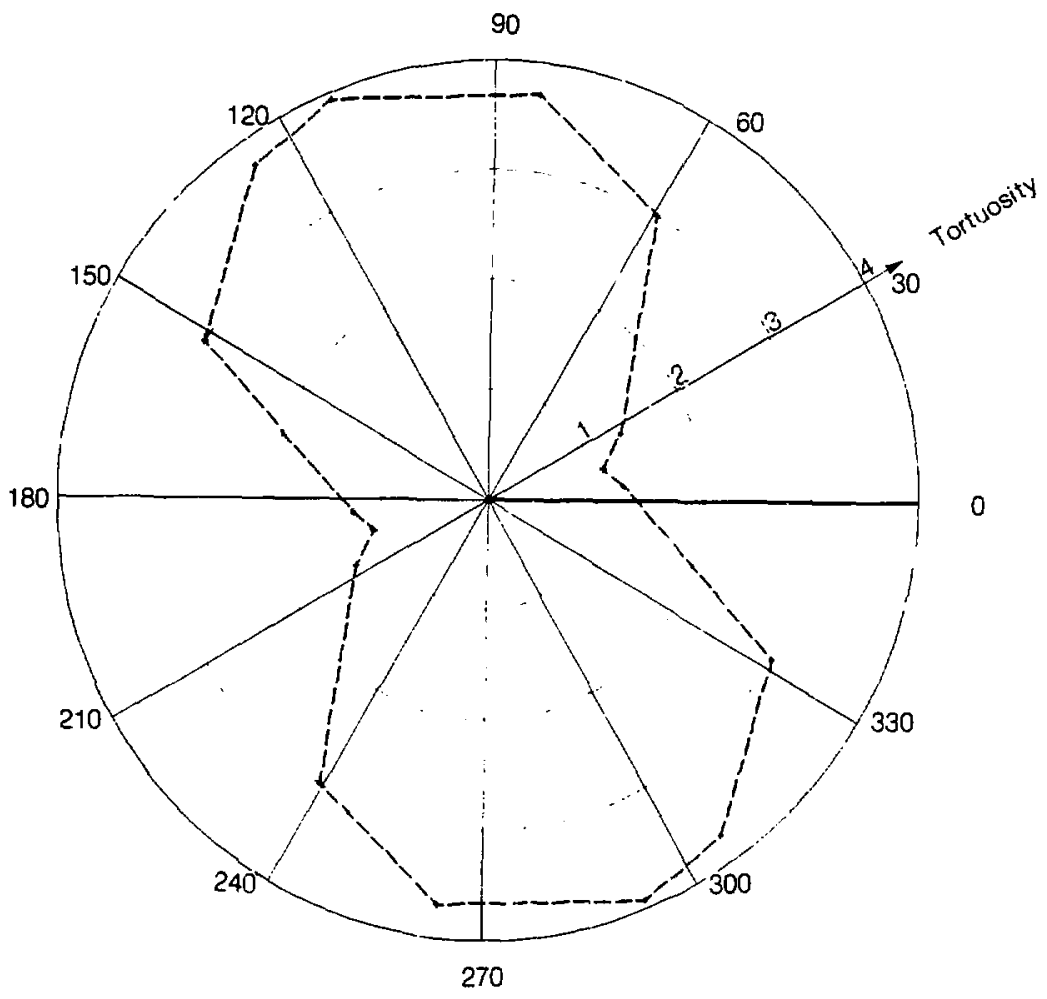

XEL839-875

Figure 6-5 Polar Plot of Tortuosity for Discontinuous Fracture System of Two Sets Oriented at $0^{\circ}$ and $30^{\circ}$. 


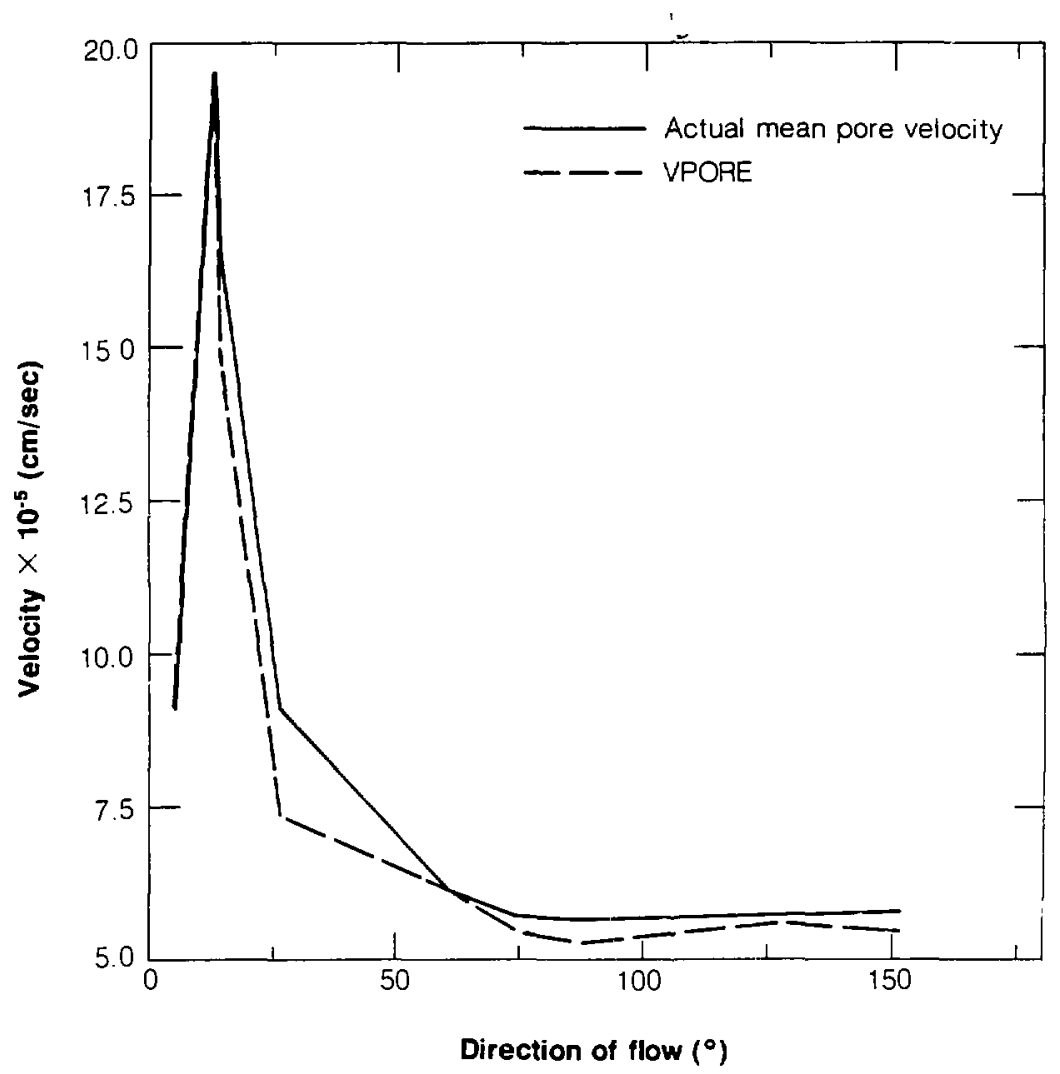

XBL839-874

Figure 6-6 Actual Mean Pore Velocity and Calculated Mean Pore Velocity for First Realization of Discontinuous Fracture System of Two Sets Oriented at $0^{\circ}$ and $30^{\circ}$. 


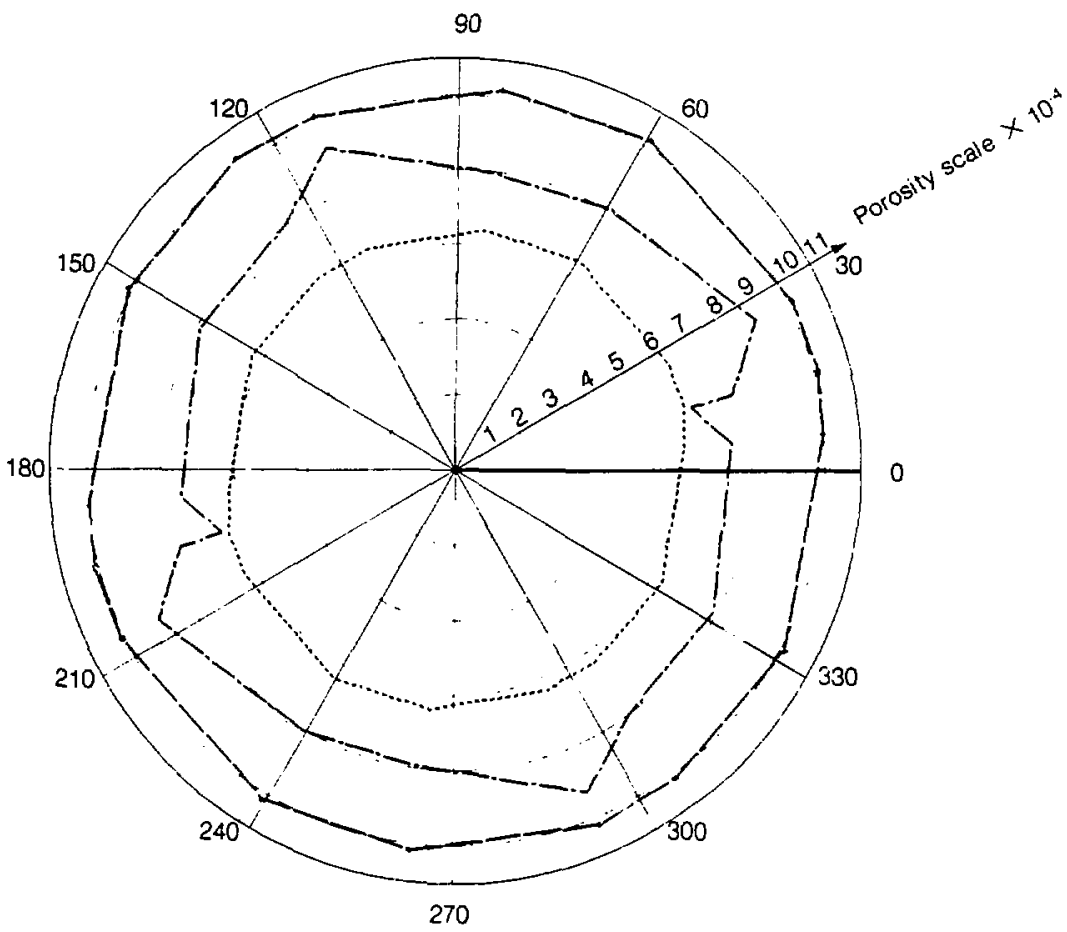

-....... Rock effective porosity

--_-- Hydraulic effective porosity

__ Total porosity

$\times 8+839-879$

Figure 6-7 Polar Plots of Total Porosity, Hydraulic Effective Porosity, and Rock Effective Porosity for Discontinuous Fracture System of Two Sets Oriented at $0^{\circ}$ and $30^{\circ}$. 
mean hydraulic effective porosity of 0.00080 is a good estimate of $\phi_{H}$ in any direction, and transport can be predicted by treating this fracture system like an equivalent porous medium.

Three composite breakthrough curves are shown in Figure 6-8. Direction of flow increases from the dirention of maximum permeability to the direction of minimum permeability as one proceeds down this figure. Near the direction of maximum permeability, the bulk of the fluid arrives at side 4 in a narrow time interval, with travel times less than $\bar{t}$. The right skewness in the curve is caused by a small part of the fluid that takes a long time to travel through the flow region. As direction of flow moves towards the direction of minimum permeability, a greater percent of the fluid have travel times larger than $\bar{t}$ as more slow zones of movement develop within the flow region. Consequently, the breakthrough curve becomes more symmetric, but the right skewness in the breakthrough curve is still evident.

Figure 6-9 shows the directional variation in the longitudinal geometric dispersivity. The maximum $\alpha_{\mathcal{L}}$ is obtained near the direction of maximum permeability. In the four directions midway between the directions of principal permeabilities, $\alpha_{L}$ decreases to minimum values. The minimum $\alpha_{\mathrm{L}}$ is seven times less than the maximum $\alpha_{\mathrm{L}}$. This strong directional dependence in $\alpha_{\mathrm{L}}$ means that this anisotropic medium cannot be treated as an equivalent isotropic medium for transport studies. The use of a directionally stable $\alpha_{L}$ for this fracture system would lead to serious errors in transport oredictions.

Thus, the following conclusions can be made about the directional properties of this fracture system. The parameters DEVA and DEVF show that porous medium equivalence is more likely to occur near the direction of maximum permeability than near the direction of minimum permeability. Hydraulic effective porosity is relatively stable with direction so that the system can be treated like an equivalent porous medium for transport. The mean $\phi_{H}$ is not equal to either $\phi$ or $\phi_{R}$, but approximately equal to the average of the two porosities. The importance of understanding the directional transport properties of this anisotropic medium is exhibited by $\alpha_{\mathfrak{L}}$. Longitudinal geometric dispersivity is highly directionally dependent with the maximum $\alpha_{L}$ being at least seven times larger than the minimum $\alpha_{L}$. 


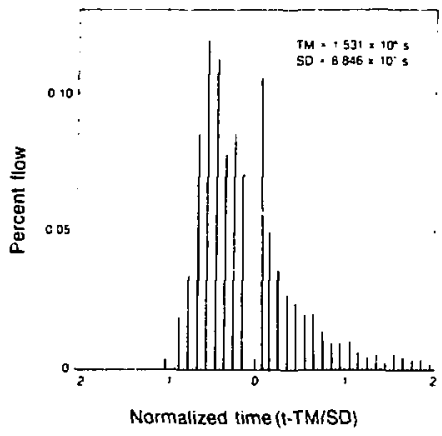

$$
\begin{gathered}
\text { Direction of } \\
\text { maximum } \\
\text { permeability }
\end{gathered}
$$

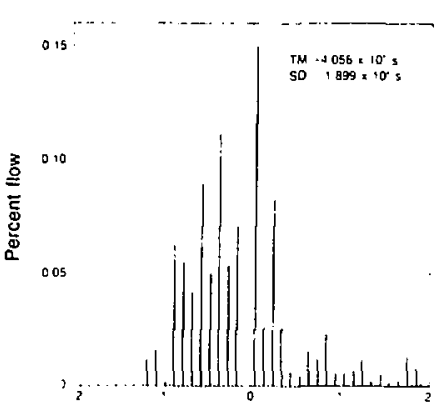

Formalized time (I-TM/SD)

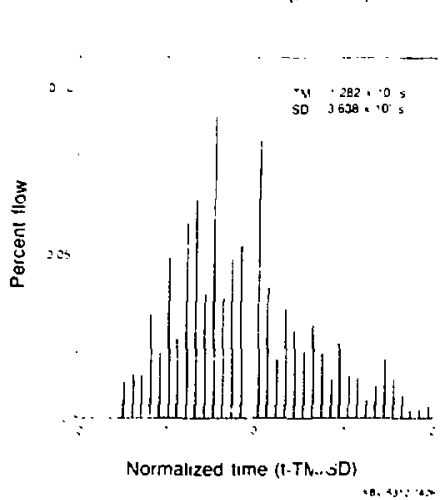

Direction

of llow

Direction af

mınımum

permeability

Figure 6-8 Three Breakthrough Curves for Directions of Flow Which Increase From the Direction of Maximum Permeability to the Direction of Minimum as One Proceeds Down the Figure. 


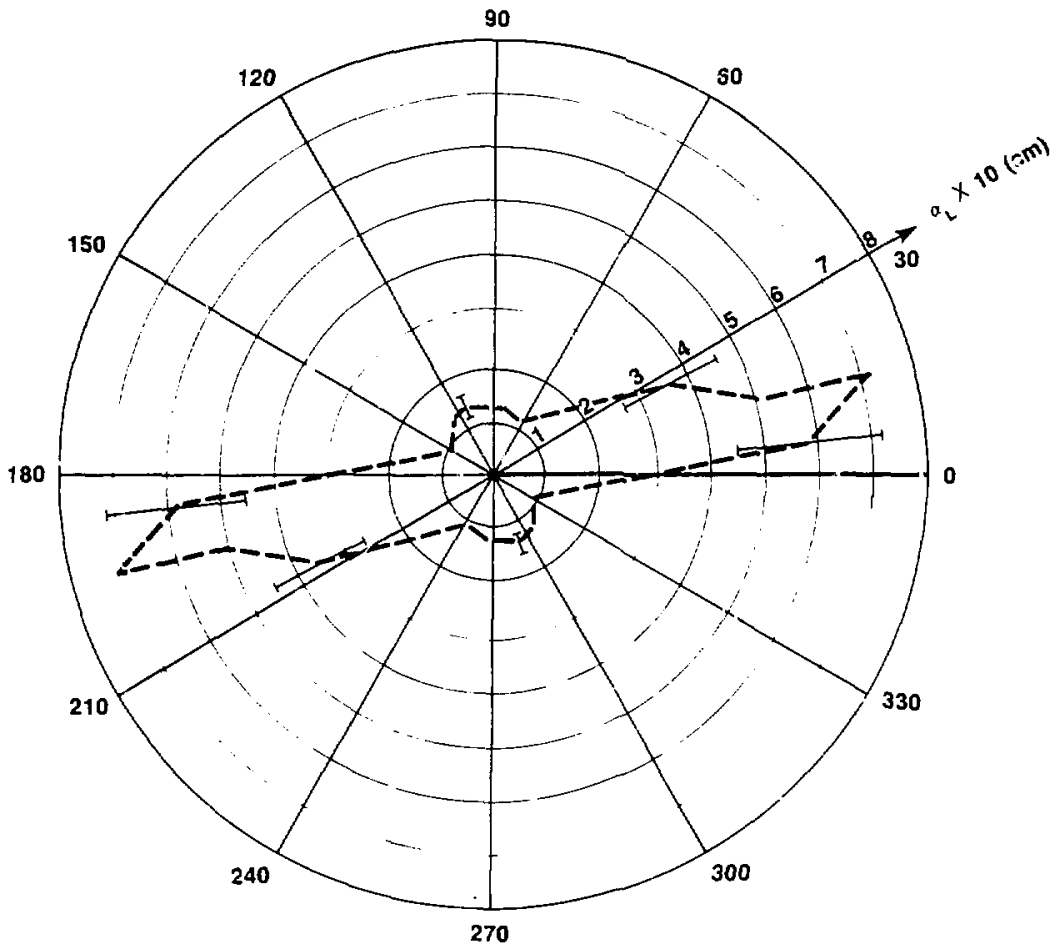

XBL 8310-330A

Figure 6-9 Polar Plot of Longitudinal Geometric Dispersivity Versus Direction of Flow for Discontinuous System of Two Sets Oriented at $0^{\circ}$ and $30^{\circ}$. 


\subsection{DISCONTINUOUS FRACIURE SYSTEM OF TWO SETS \\ ORIENTED AT $0^{\circ}$ AND $60^{\circ}$}

The first Monte Carlo study was conducted for a discontinuous fracture system in which the three geometric parameters length (l), aperture (b), and orientation (o) were all constant. The following Monte Carlo study was conducted for a fracture system in which the three geometric parame:ers were all probabilistically simulated from the mean $(\mu)$ and the standard deviation $(\sigma)$ for each parameter. The fracture system consisted of the following geometric parameters:

$$
\begin{aligned}
& \mu_{0, \text { set l }}=0^{\circ}, \quad \sigma_{0, \text { set } 1}=5^{\circ} \\
& H_{0 \text { ant }} \lambda_{1}=60^{\circ}, \sigma_{0, v e 12}=5^{\circ} \\
& \mu_{1}=40 \mathrm{~m}, \quad \sigma_{1}=4 \mathrm{~m} \\
& \mu_{\mathrm{b}}=0.00002 \mathrm{~m}, \quad \sigma_{\mathrm{b}}=0.000002 \mathrm{~m}
\end{aligned}
$$

Ten orientations of the hydraulic gradient were selected for study in each realization. These oriesitations wre selected in order to obtain a representative sample of mechanical transport in all directions. Direction of flow for each orientation was estimated based on calculations made for a continuous fracture system of two parallel sets of fractures oriented at $0^{\circ}$ and $60^{\circ}$. Each fracture in this continuous system had the same aperture, and the spacing betwee $\pi$ fractures of the same set was constant. The ten orientations and estiriated directions of flow are listed in Table 6-2. The estimated directions of naximum and minimuzt permeabilities are $30^{\circ}$ and $120^{\circ}$, respectively. The estimated ratio of $\mathrm{K}_{\mathrm{r}}$ to $\mathrm{K}_{\mathrm{y}}$ is two. Flow regions of size 160 by $160 \mathrm{~m}$ (size limited by computer storage) were used for all oneitations, and the hydraulic gradient along sides 1 and 3 was set at 0.01 for all flow rezions. Figure 6-10 shows the fracture pattern in the generation region of 500 by $500 \mathrm{~m}$ for one of the realizations. Figure 6-11 shows the fracture pattern and conductive fracture segments in a flow region oriented at $30^{\circ}$ for the same realization. 


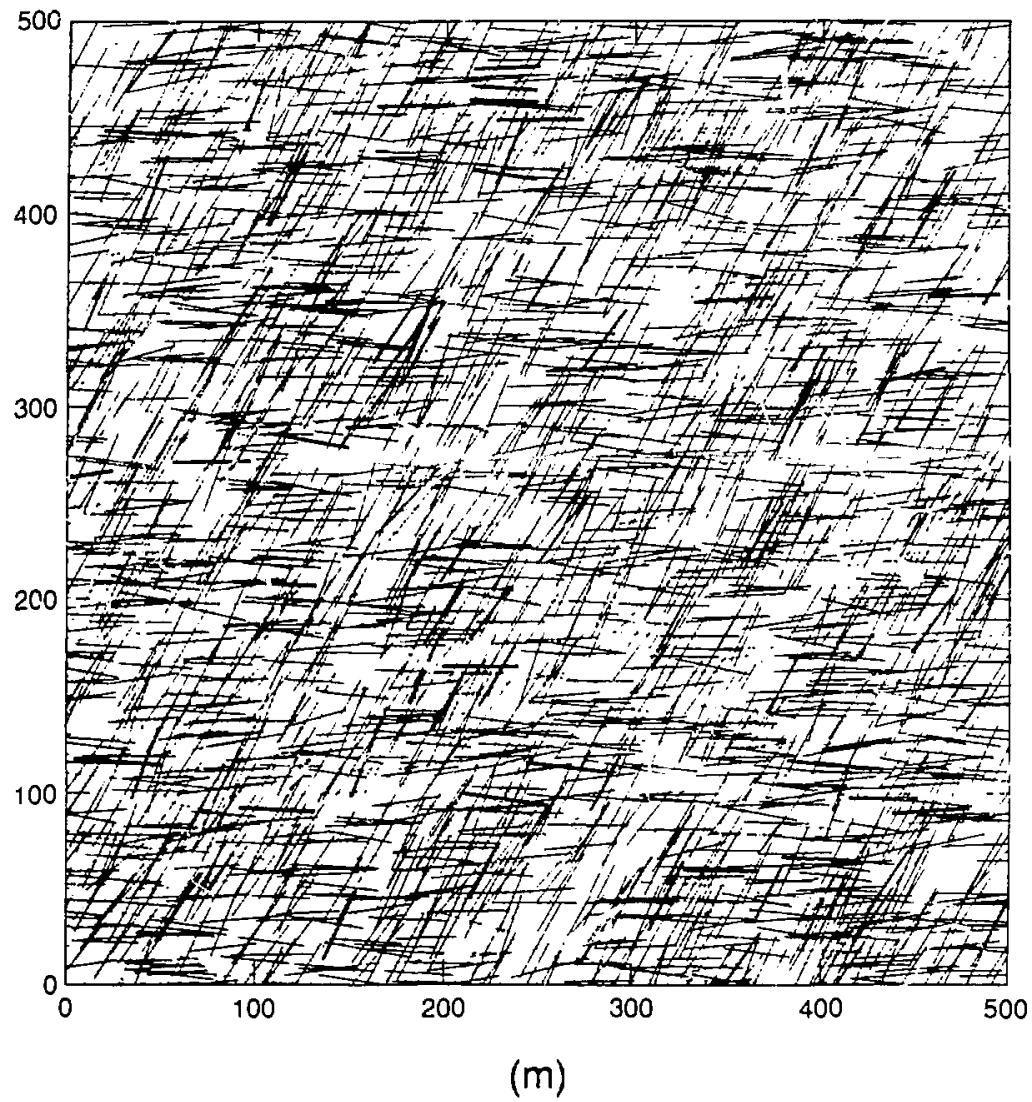

YBL $3+1-390$

Figure 6-10 Fracture Network in the Generation Region for Discontinuous System of Two Sats Oriented at $0^{\circ}$ and $60^{\circ}$. 

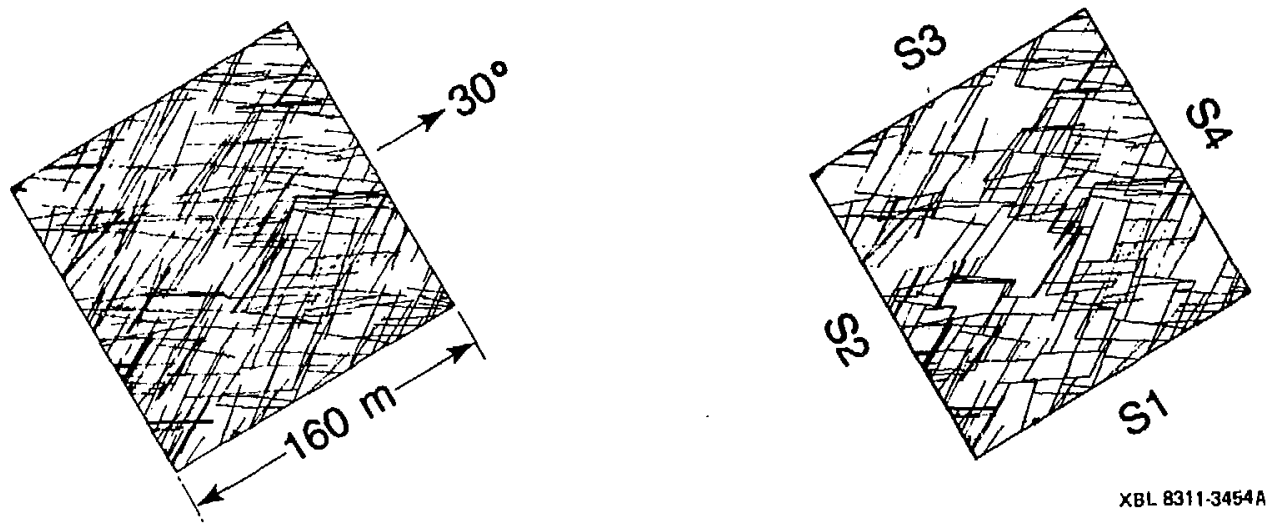

Figure 6-11 Networks of a) Fractures and b) Connected Fracture Segments in the Flow Region Oriented at $30^{\circ}$ for Discontinuous System of Two Sets Oriented at $0^{\circ}$ and $60^{\circ}$. 


\begin{tabular}{|c|c|c|}
\hline \multicolumn{3}{|c|}{$\begin{array}{l}\text { Table 6-2. Orientations and Estimated Directions of Flow Used in Monte } \\
\text { Carlo Simnlation of the Disconianuous System Consisting } \\
\text { of Tro Sets of Fractures Oriented at } 0^{\circ} \text { and } 60^{\circ} \text {. }\end{array}$} \\
\hline $\begin{array}{l}\text { Orientation } \\
\text { of hydraulic } \\
\text { gradient } \\
\text { degrees } \\
\end{array}$ & $\begin{array}{l}\text { Angle of Flow based } \\
\text { on continuous } \\
\text { system } \\
\text { degrees }\end{array}$ & $\begin{array}{c}\text { Estimated Direction } \\
\text { of flow } \\
\text { degrees }\end{array}$ \\
\hline $\begin{array}{c}30 \\
75 \\
95 \\
107 \\
114 \\
120 \\
126 \\
138 \\
145 \\
165\end{array}$ & $\begin{array}{c}0 \\
-26.6 \\
-29.4 \\
-21.7 \\
-11.5 \\
0 \\
11.5 \\
21.7 \\
29.4 \\
26.6\end{array}$ & $\begin{array}{c}30 \\
48.4 \\
65.6 \\
85.3 \\
102.5 \\
120 \\
137.5 \\
159.7 \\
167.4 \\
191.6\end{array}$ \\
\hline
\end{tabular}

The stability of hydraulic effective porosity determined the number of realizations for this Monte Carlo study. Figure 6-12 shows the mean hydraulic effective porosity for three orientations $\left(30^{\circ}, 75^{\circ}\right.$, and $\left.120^{\circ}\right)$ piotied against the number of realizations. Orientations $30^{\circ}$ and $120^{\circ}$ were chosen because they were aligned in the estimated directions of principal permeabilities. For orientations $75^{\circ}$ and $120^{\circ}$, wean $\phi_{H}$ fluctuated in the first ten realizations. However, mean $\phi_{\mathrm{H}}$ was relatively stable for all three orientations after twelve realizations, and a slight directional dependence in $\phi_{\mathrm{H}}$ was apparent. This Monte Carlo study ended after the seventeenth realization because hydraulic effective porosity in each of the three orientations was stable.

Figure $6-13$ shows the plot of mean $\sqrt{K_{f}}$ versus direction of flow. The standard error of the mean $\sqrt{K_{1}}$ was less than 2.5 percent of its mean in any direction. The plot of the mean $\sqrt{\mathrm{K}_{\mathrm{f}}}$ can be approximated by an ellipse with directisns of maximum and minimum permeabilities near $30^{\circ}$ and $120^{\circ}$, respectively. This curve is nearly symmetric about the direction of minimum permeauility, and the ratio of $K_{-x}$ to $K_{y}$ is about 2.4. The elliptic shape of the $\sqrt{K_{f}}$ curve shows that the directional flow characteristics for this fracture system behaved like an equivalent porous medium. Table 6-3 lists the computed mean direction of flow and the standard error of the mean for each orientation of the hydraulic gradient. The fluid flow calculations made for the cr.titinunus fracture system gave good estimates of the fluid flow properties 

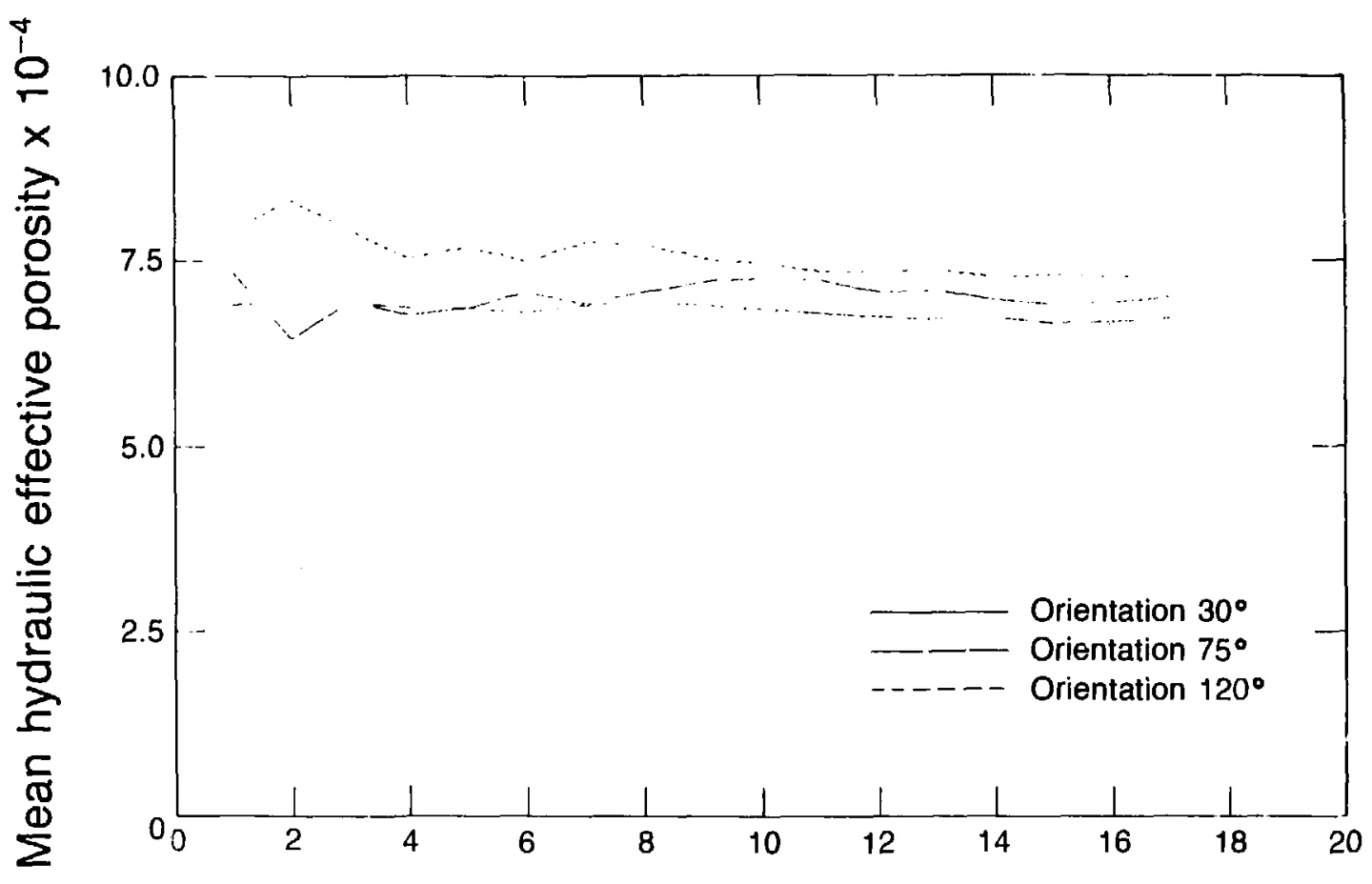

\section{Number of realizations}

Figure 6-12 Mean Hydraulic Effective Porosity for Orientations $30^{\circ}, 75^{\circ}$, and $120^{\circ}$ Versus the Number of Realizations. 


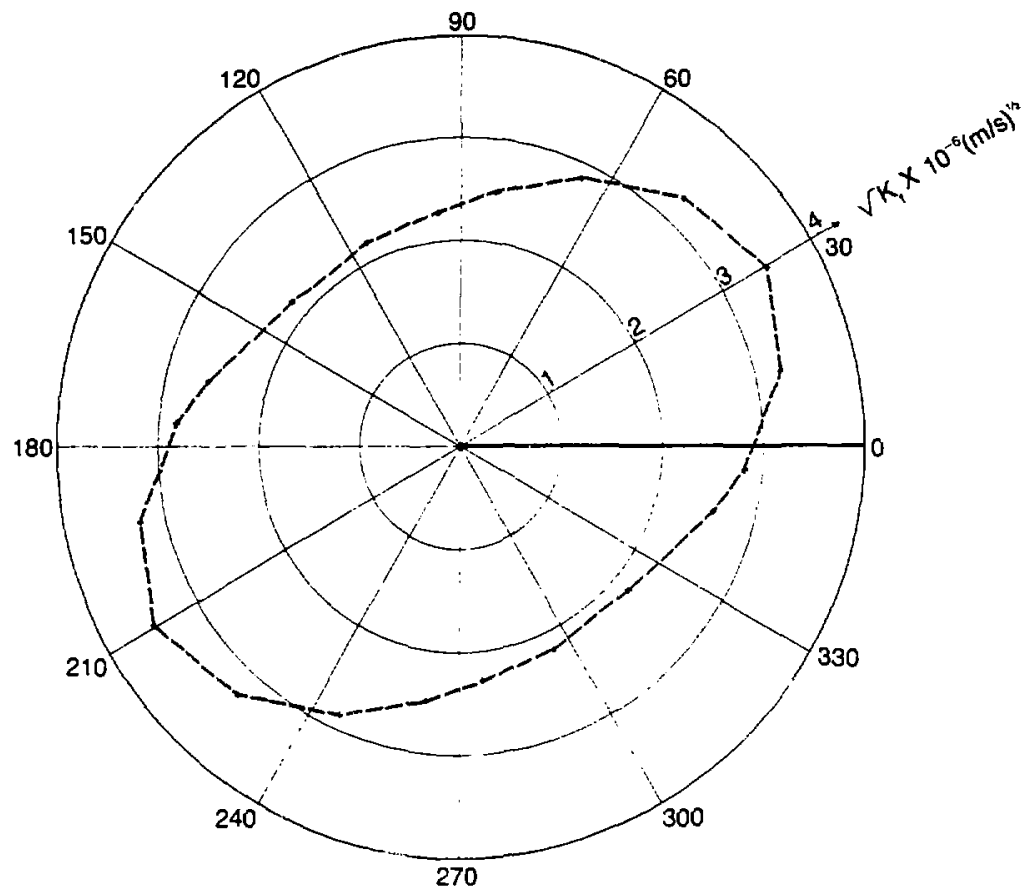

$X B L \quad 841-530$

Figure 6-13 Polar Plot of Square Root of Permeability in Direction of Flo'v for Discontinuous System of Two Sets Oriented at $0^{\circ}$ and $60^{\circ}$. 


\begin{tabular}{|c|c|}
\hline $\begin{array}{r}\text { Table 6-3. Orientations and Directions of Flow Calculated for } \\
\text { Monte Carlo Study of the Discontinucus System } \\
\text { of Two Sets of Fractures Oriented at } 0^{\circ} \text { and } 60^{\circ}\end{array}$ \\
\hline $\begin{array}{l}\text { Orientation } \\
\text { of hydraulic } \\
\text { gradient } \\
\text { degrees }\end{array}$ & $\begin{array}{c}\text { Direction } \\
\text { of flow }\end{array}$ \\
\hline & degrees \\
30 & \\
75 & $29.69 \pm 2.05$ \\
95 & $47.33 \pm 0.62$ \\
107 & $65.37 \pm 1.02$ \\
114 & $81.96 \pm 1.16$ \\
126 & $95.96 \pm 1.82$ \\
126 & $115.38 \pm 2.90$ \\
138 & $140.00 \pm 2.06$ \\
145 & $165.74 \pm 0.81$ \\
165 & $175.25 \pm 0.58$ \\
& $192.91 \pm 0.46$ \\
\hline
\end{tabular}

for this system.

Mean DEVA and mean DEVF for this fracture system showed the same directional behavior (Figure 6-14) as the previous discontinuous system (Figure 6-4). DEVA exhibited two peaks, one near the direction of maximum permeability and the other near the direction of minimum permeability. The flow field was less uniform near the directions of principal permeabilities than in directions away from the principal directions. DEVF was inversely related to permeability, as DEVF steadily increased from the direction of maximum permeability to the direction of minimum permeability. Flow rate was large and fluid flowed easily in the direction of maximum permeability, as tortuosity was minimum (Figure 6-15), and DEVF was small. In the direction of maximum permeability, fluid flowed in a direction close to the orientations of the two sets. However, in the direction of minimum permeability, the orientation of the hydraulic gradient was not aligned favorably wjth the grientations of the sets. The mean orientations of both sets were not oriented in the direction of the hydraulic gradient, and fluid had to move in a particular direction that was controlled by the orientation of the hydraulic gradient. Tortuosity and DEVF were large in the direction of minimum permeability because fluid flowed in a direction nearly perpendicular to the orientations of the two sets.

Equivalent porous medium flow behavior for this fracture system is directional 


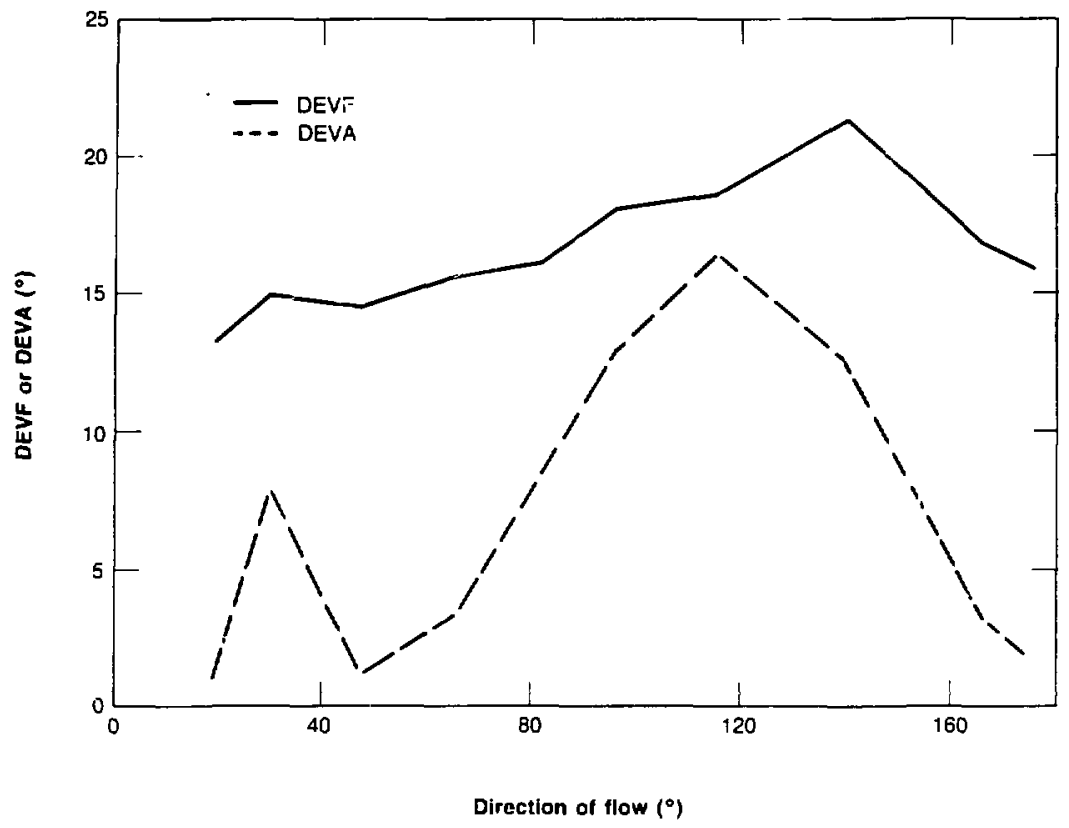

XBL $8401-6786$

Figure o-14 Mean DEVF and Mean DEVA V'ersus Direction of Flow for Discontinuous System of Two Sets Oriented at $0^{\circ}$ and $60^{\circ}$. 


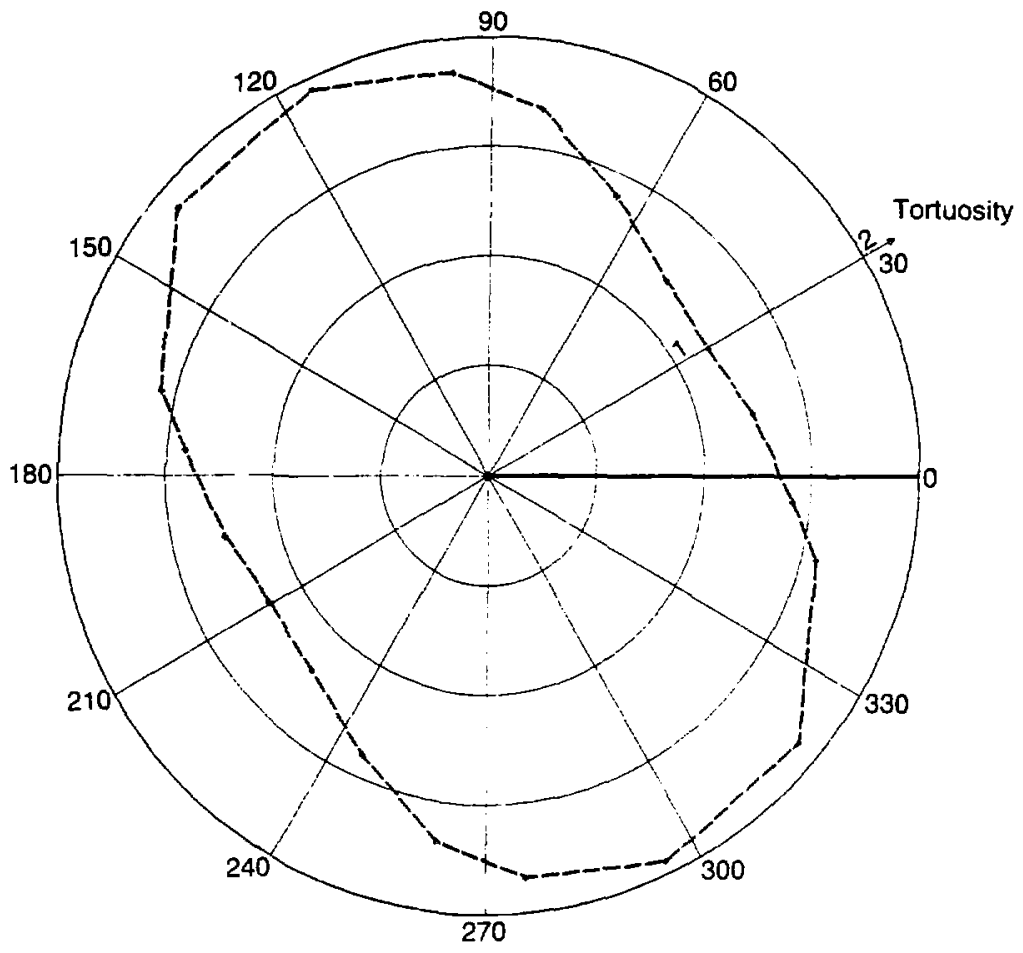

XBL B401-6795

Figure 6-15 Polar Plot of Tortuosity for Discontinuous System of Two Sets Oiented at $0^{\circ}$ and $60^{\circ}$. 
dependent. Flow characteristics are better predicted by Darcy's law when the direction of flow is near the direction of maximum permeability than when it is near the direction of minimum permeability, as evidenced by the smaller DEVF and DEVA near the direction of maximum permeability. Since permeability is inversely proportional to tortuosity, the study of the fluid flow characteristics for this fracture system has shown that fluid flow continuum size is inversely related to tortuosity. The study of mechanical transport for the continuous fracture system oriented at $0^{\circ}$ and $30^{\circ}$ showed that mechanical transport continuum size was also inversely related to tortuosity (section $\mathbf{5 . 2}$ ).

Next, the relationship between mean pore velocity and VPORE was investigated. The estimate of mean pore velocity, VPORE, discussed in section 4.4 , can be derived from the Kozeny equation (section 3.8). Kozeny formulated an expression relating pipe flow to porous media flow. The mean velocity in a pipe under laminar flow conditions is given by the Poiseuille equation:

$$
\bar{v}=-\frac{r_{h}^{2}}{2 \mu} \frac{d p}{d L}
$$

The first key step in the formulation of the Kozeny equation was the acceptance that the Poiseuille equation was valid for porous media flow, with a few added modifications. The first modification accounted for the fact that fluid flows in only part (the conductive void region) of the total volume. The second modification accounted for the fact that fluid paths are nonlinear in a porous medium. The next key step in the formulation of the Kozeny equation was the development of the relationship between MPV and specific discharge. The mean pore velocity is expressed in the Kozeny equation as:

$$
M P V=\frac{q}{\phi} \frac{L_{2}}{L}=-\frac{r_{l}^{2}}{\mu S_{t}} \frac{d p}{d L_{2}}
$$

Theoretically,

$$
M P V=\frac{1}{V_{c}} \int_{V_{s}}{ } d V_{c}
$$

which is a very difficult equation to evaluate. In equation 6.1 , mean pore velocity is related to macroscopic parameters ( $q, \phi, L$ and $L_{\mathbf{z}}$ ) which are much easier to compute than is equation 
6.2. Since $L_{2} / L$ is the tortuosity, mean pore velocity can be written as:

$$
\mathrm{MPV}=\frac{\mathrm{q}}{\phi}
$$

which for bomogeneous transport is equal to:

$$
\text { MPV }=\text { VLIN } t=\text { VPORE }
$$

Thus, VPORE is a function of two mechanical transport parameters that are measured in this study.

Tortuosity increased from 1.17 near the direction of maximum permeability to 1.95 near the direction of minimum permeability. The relationship between VPORE and mean pore velocity was investigated in three directions near the direction of minimum permeability, where tortuosity has a definite effect of VPORE. For the three directions of $82.0^{\circ}, 96.0^{\circ}$, and $115.4^{\circ}$, mean tortuosities were $1.68,1,80$, and 1.95 , respectively. Mean MPVs of $1.275 \times 10^{-6}$, $1.265 \times 10^{-6}$, and $1.248 \times 10^{-6} \mathrm{~m} / \mathrm{s}$ were calculated in these three directions, respectively. Mean VPOREs of $1.234 \times 10^{-6}, 1.224 \times 10^{-6}$, and $1.240 \times 10^{-6} \mathrm{~m} / \mathrm{s}$ were also calculated in the sa. $\cdot$ : three directions, respectiveiy. VPORE slightly underestimated MPV in the three directions. However, the difference between VPORE and MPV was less than 4 percent of MPV. Thus, VPORE provided a good estimate of the mean pore velocity.

Tortuosity has been found to be an important mechanical transport parameter. Tortuosity, as just shown, is an essential component in estimating VPORE. The continuum sizes for mechanical transpon and fluid flow were found to be inversely related to tortuosity. Tor:uosity is normally considered to range between 1 and 2 . However, for the continuous and discontinuous fracture systems of two sets oriented at $0^{\circ}$ and $30^{\circ}$, tortuosities as high as 3.8 were calculated. Frs discontinuous systems which exhibited continua behavior for directional fluid flow, tortuosity increased from a minimum value in the direction of maximum permeability to a maximum value in the direction of minimum permeability, as one would expect for equivalent porous media.

Total porosity, rock effective porosity, and hydraulic effective porosity are each plotted against direction of flow in Figure 6-15. Total porosity and rock effective porosity were both 

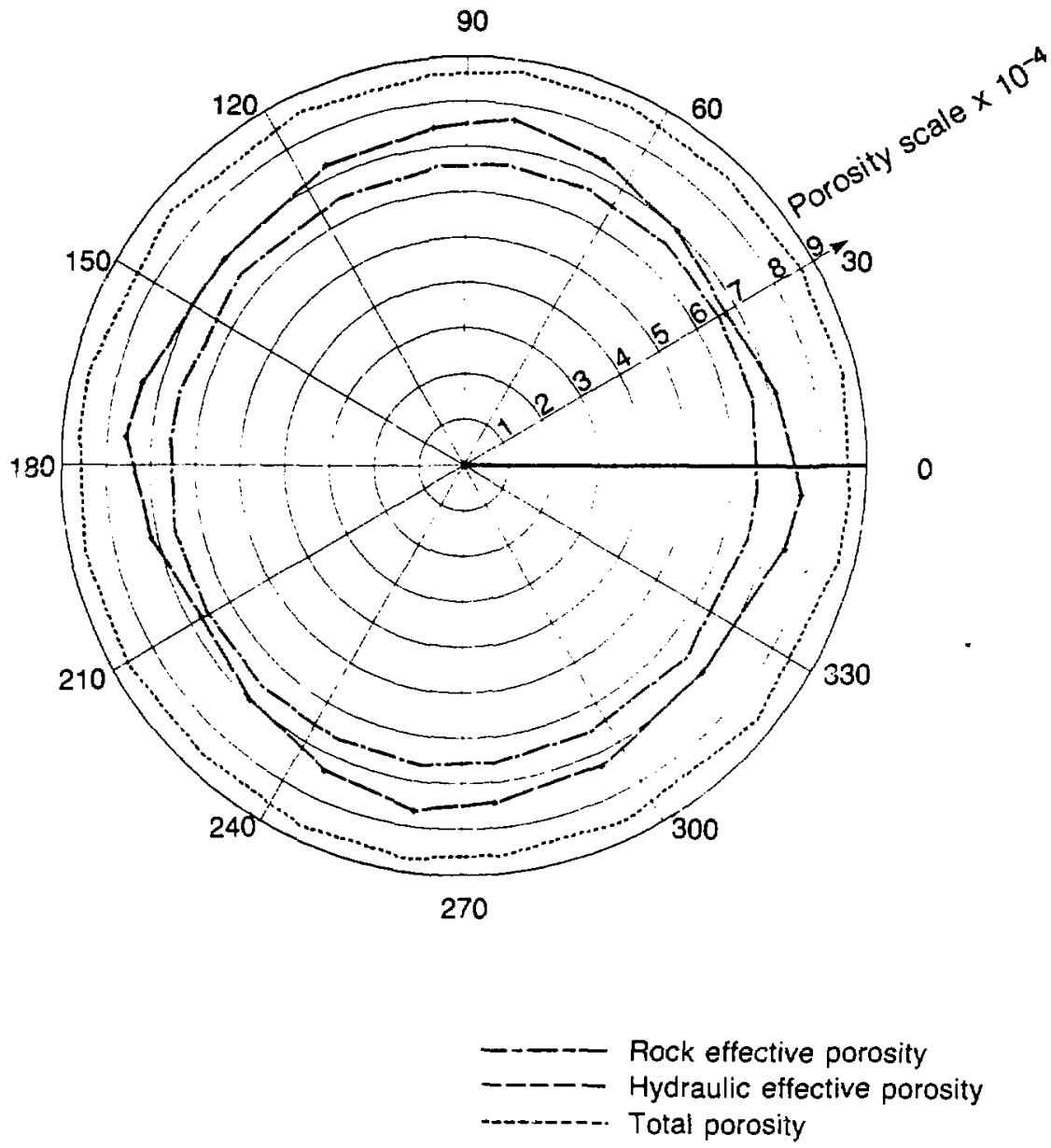

XBL 8311.7383

Figure 6-16 Polar Plots of Rock Effective Porosity, Hydraulic Effective Porosity, and Total Porosity for Discontinuous System of Two Sets Oriented at $0^{\circ}$ and $60^{\circ}$. 
stable with direction. Hydraulic offective porosity was slightly larger than $\phi_{R}$, and the mean $\phi_{H}$ was 0.00000727 . Hydraulic effective porosity showed a small directional dependence, with minimum $\phi_{\mathrm{H}}$ sccurring near the direction of maximum permeability, and the maximum $\phi_{\mathrm{H}}$ occurring midway between the directions of principal permeabilities. The small directional dependence in hydraulic effect:ve porosity indicates that this fracture system can be treated like an equivalent porous medium for transport.

The two Monte Carlo studies for discontinuous fracture systems showed that hydraulic effective porosity was larger than $\phi_{R}$, but less than $\phi$. Thus, $q / \phi_{R}$ would overestimate the average linear yelocity for these two systems. Hydraulic effective porosity was largar than $\phi_{R}$ because zones of slow movement exist in the flow region which caused $\bar{t}$ to be larger than theoretically expected. Hydraulic effective porosity exhibited a slight directional dependence, with the minimum $\phi_{H}$ occurring near the direction of maximum permeability. The abrupt changes in $\phi_{\mathbf{H}}$ found in the regular continuous fracture systems were not observed in the discontinuous systems.

The directional variation of $\alpha_{L}$ for this fracture system is shown in Figure 6-17. The maximum $\alpha_{\mathbf{L}}$ was obtained at a direction midway between the two directions of principal permeabilities. The minimum $\alpha_{\mathrm{L}}$ was obtained near the direction of minimum permeability. The ratio of maximum to minimum $\alpha_{\mathrm{L}}$ was four.

The following summarizes the results of the directional studies for the longitudinal geometric dispersivity. For the first continuous fracture systern of two parallel sets oriented at $0^{*}$ and $30^{\circ}$, the maximum $\alpha_{\mathrm{L}}$ was obtained in the direction of minimum permeability. However, the minimum $\alpha_{\mathbb{L}}$ of zero was obtained in the two principal directions for the next continuous system of two orthogonal sets. The discontinuous fracture system of two sets oriented at $0^{\circ}$ and $30^{\circ}$ had the maximum $\alpha_{\mathrm{L}}$ in the direction of maximum permeability, and the minimum $\alpha_{\mathrm{L}}$ at a direction midway between the two principal directions. For the discontinuous system just studied, the maximum $\alpha_{\mathrm{L}}$ occurred at a direction midway between the two principal directions, and the minimum on occurred near the direction of minimum permeability. Thus, each system 


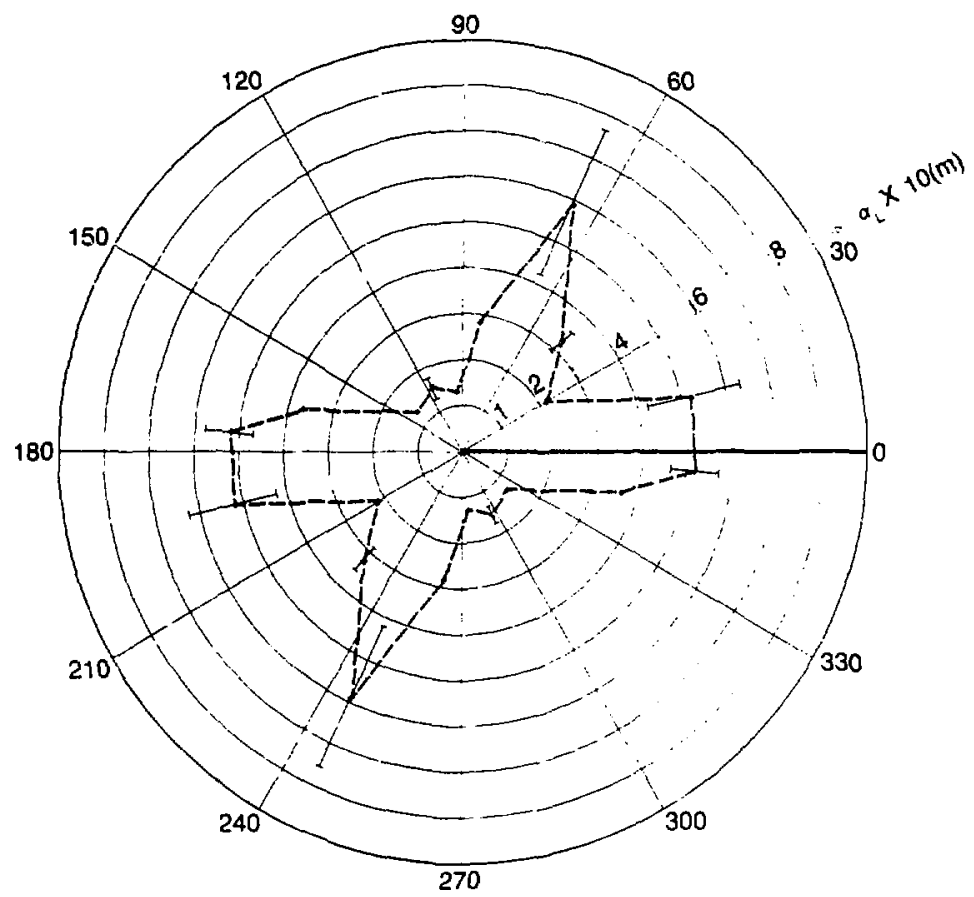

$\times 8 L 841-531$

Figure 6-17 Polar Plot of Longitudinal Geometric Dispersivity for Discentinuous System of Two Sets Oriented at $0^{\circ}$ and $60^{\circ}$. 
showed a unique directional dependence for $\alpha_{\mathrm{L}}$. The ratio of $\alpha_{\mathrm{L}, \max }$ to $\alpha_{\mathrm{L}, \min }$ for the two discontinuous systems was related to the degree of anisotropy (ratio of $K_{\mathbf{x}}$ to $\mathrm{K}_{\mathbf{y}}$ ).

The longitudinal geometric dispersivity will not vary with flow region size if the Fickian appruach to characterizing dispersion is applicable. The applicability of the Fickian approach was studied using the fracture system in the last realization, as follows. The width of a square flow region oriented at $107^{\circ}$ was increased in increments of $20 \mathrm{~m}$, beginning at a width of $40 \mathrm{~m}$. Orientation $107^{*}$ was selected for this study because the fluid flow and mechanical transport parameters ( $K_{f}, \phi_{\mathrm{H}}, \tau, \alpha_{\mathrm{L}}$ ) for the last realization were all reasonably close to their mean values for the Monte Carlo study. For each flow region, the computer program was used to determine $\alpha_{\mathrm{L}}$. Computer storage limited the maximum width of the flor + gion to $160 \mathrm{~m}$.

Figure 6-18 shows the variation in $\alpha_{\mathrm{L}}$ with sample size. Since $\alpha_{\mathrm{L}}$ increases with sample size, the Fickian approach cannot be used to characterize dispersion at this scale. The polar plot of the longitudinal geometric dispersivity shown in Figure 6.17 can only be used to predict transport for problems on the scale of $160 \mathrm{~m}$. For problems on a larger scale, $\alpha_{\mathrm{L}}$ is expected to be larger than $\alpha_{\mathrm{L}}$ in Figure 6-17. Longitudinal geometric dispersivity vaies linearly with size when the width of the flow region is greater than $75 \mathrm{~m}$. Linearly varying dispersivities have been reported in the literature. Pickens and Grisak (1981a) had good success in matching the results of tracer experiments by Sudicky and Cherry (1979) and Pickens and Gisak (1981b) with linearly varying dispersivities. Presently, most tiansport models are based on the Fickian approach. However, the results of this study agree with a number of recent studies (Gelhar et al., 1979: Pickens and Grisak, 1981; Schwarz, 1977) which have demonstrated that dispersion coefficients initially increase with sample size.

\subsection{SENSITIVITY ANALYSIS}

A fractuze systcm is created from the following geometric parameters: aperture (b), orientation (o), lengih (1), and set areal density $\left(\lambda_{N}\right)$. The first three geometric parameters are gen* erally distributed and are modeleo in this study using probabilistic simulation. This statistical procedure requires a knowledge of the mean and standard deriation for the simulated 


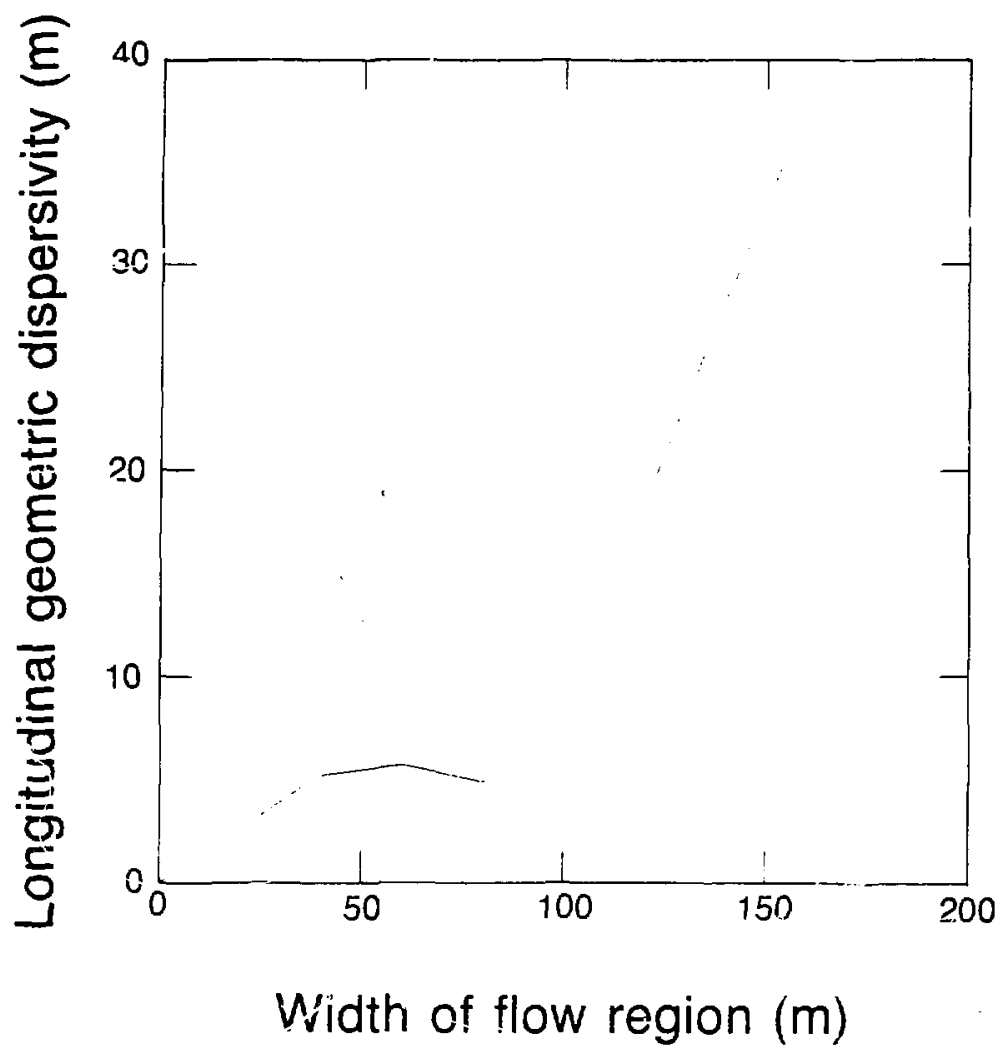

XBL $841-391$

Figure 6-18 Longitudinal Geometric Dispersivity Versus Widih of Flow Region for Discontinuous System of Two Sets Oriented at $0^{\circ}$ and $60^{\circ}$. 
distribution. A meclanical transport or fluid flow parameter, say $\phi_{\mathrm{H}}$, can be expressed mathematically in terms of the fracture geometry in the following way:

$$
\phi_{\mathrm{H}}=\mathbf{f}\left(\mu_{\mathrm{b}}, \sigma_{\mathrm{b}}, \mu_{0}, \sigma_{0}, \mu_{\mathrm{b}}, \sigma_{\mathrm{b}}, \lambda_{\mathrm{A}}\right)
$$

This analysis investigates the sensitivity of mechanical transport and fluid flow parameters with respect to perturbations in each geometric parameter listed in the equation above. Field engineers will find this information valuable when measuring and collecting fracture data. Careful and accurate measurement must be made ${ }^{f}$ - a geometric parameter which exhibits high sensitivity to Iuid flow and mechanical transport. The sensitivity study is presented in two parts. The first part evaluates the sensitivity of mean gesmetric parameters ( $\mu$ ). Part two investigates the effect the standard deviation $(\sigma)$ of each geometric parameter has on mechanical transport and fluid flow.

\subsubsection{Sensitivity of Mean Geometric Parameters and Set Areal Density}

The sensitivity study of the mean geometric parameters investigates the changes in mechanical transport and fluid flow parameters with respect to perturbatiors in each mean geometric parameter. The mean geometric parameters are $\mu_{b}, \mu_{l}$, and $\mu_{0}$. The sensitivities for $\mu_{1}, \mu_{0}$ and $\lambda_{A}$ were studied together because the three geometric parameters influence the connectivity of a fracture network. Connectivity is the number of connected (nonisclated) fracture intersections per volume of rock. Connectivity affects both the amount of fluid that can flow through the fracture network (conductivity) and the paths of fluid movement in the network. The sensitivity of mean aperture was studied separately because mean aperture influences only the conductivity of a fracture network. No change in the connectivity of the network occurs when mean aperture is perturbed.

The sensitivity study of a mean geometric parameter proceeded as follows. Fracture systems were created in which the sisdied mean geometric parameter was systematically perturbed from its initial value in the control fracture system, while keeping the remaining geometric parameters constant. Thus, sensitivity was measured over a range of values for the studied geometric parameter. A common control fracture system was used in each sensitivity study of 
a mean geometric parameter. The fracture system in the first realization of the Monte Carto simulation for the discontinuous fracture system of two sets oriented at $0^{\circ}$ and $30^{\circ}$ served as the controi fracture system. This fracture system had the following constant fracture geometric parameters: $40 \mathrm{~cm}$ length, $0.002 \mathrm{~cm}$ aperture, $0^{\circ}$ orientation for set $1,30^{\circ}$ orientation for set 2 , and $0.00633 \mathrm{~cm}^{-2}$ areal density for each set. For each perturbed fracture system, measurements of mechanical transport and fluid flow were made for a flow region of $180 \times 180 \mathrm{~cm}$ oriented at $160^{\circ}$. This orientation was selected because it showed a good probability of exhibiting equivalent porous meuium flow behavior in the Monte Carlo study. The results for the perturbed fracture systems were then analyzed to evaluate sensitivity.

The connectivity sensitivity studies for mean orientation, set areal density, and mean fracture length were conducted with the following perturbed fracture systems. In the orientation sensitivity study, the orientation of set 2 was perturbed while the orientation of set 1 remained constant at $0^{\circ}$. Four orientations for set 2 of $26^{\circ}, 28^{\circ}, 32^{\circ}$, and $34^{\circ}$ were used in this part of the study. The areal density sensitivity study was conducted by equally perturbing $\lambda_{A}$ for both sets. The control fracture system had 570 fractures of each set in the generation region of $300 \times 300$ cm. In the areal density sensitivity study, the number of fractures per set in the generation region ranged from 300 to 625 fractures. In the mean fracture length sensitivity study, five values of mean fracture length ranging from 30 to $45 \mathrm{~cm}$ were used.

Two per:urbed systems were sufficient to conduct the conductivity sensitivity study for mean axerture because mean aperture is the only geometric parameter for which sensitivity can be computed. The two perturbed systems had mean apertures of $0.001 \mathrm{~cm}$ and $0.003 \mathrm{~cm}$.

\subsubsection{Connectivity Sensitivity Studies of Mean Fracture Length, Mean Orientation, and Set Areal Dersity}

The sensitivity studies for $\mu_{l}, \mu_{0}$, and $\lambda_{A}$ were conducted together because the three parameters influence the connectivity of a fracture network. Connectivity clearly decreases as set areal density : $:$ fracture length decreases. The mean orientation of fracture sets can also influence connectivity. For example, consider a fracture system consisting of two fracture sets. The 
minimum connectivity occurs when the two sets are parallel, and the maximum connectivity cccurs wivn the two sets are orthogonal.

Mean sensitivity and relative sensitivity were computed to evaluate the sensitivity of a mean geometric parameter. The relative sensitivity (S) of a mechanical transport or fluid flow parameter ( $y$ ) for a given value of a mean geometric parameter $(x)$ was computed as:

$$
S=\frac{\frac{1}{y} \partial y}{\frac{1}{x} \partial x} \approx \frac{\frac{1}{y} \Delta y}{\frac{1}{\bar{x}} \Delta x}
$$

The relative sensitivity was defined so that the seusitivities of $\mu_{l}, \mu_{0}$, and $\lambda_{A}$ could be compared on an equivalent dimensioniess basis. Relative sensitivity was computed for each perturbed value of the studied geometric parameter. For the range of the studied geometric parameter $\left(x_{1}, x_{2}\right)$, mean sensitivity was computed as:

$$
S_{\mathrm{m}}=\frac{1}{x_{2}-x_{1}} \cdot \int_{x_{1}}^{x_{1}} S d x
$$

The relative sensitivities and mean sensitivity for a range of $x$ were used to interpret a sensitivity study. Three types of relationships between $y$ and $x$ were observed. In the first type of relationship, $y$ showed a tendency to increase or decrease with respect to $x$ (solid line in Figure 6-19). Since relative sensitivities had the same sign (positive or negative), y was directly related to $\mathrm{x}$. One can predict what will happen to $\mathrm{y}$ when $\mathrm{x}$ is perturbed for this type of relationship. In the second relationship, $y$ was insensitive to $x$ (dashed line in Figure 6-19). The relative sensitivities and mean sensitivity were small in magnitude, and the relative sensitivities had mixed signs. Even if there is a small error perturbation) in the mean geometric parameter, $y$ can be predicted fairly accurately. In the third relationship, $y$ was highly sensitive to $x$ (dotted line in Figure 6-19): y fluctuated with $\pi$ and no general tendency was observed. This geometric parameter must be determined accurately to predict $y$.

Table 6-4 lists the mean sensitivity and maximum magnitude of relative sensitivity (max $|S|$ ) for the connectivity sensitivity study. Tctal porosity and rock effective porosity increased as fracture length and set areal density increased (Figure 6-20). The increase in both porosities reflected increases in connectivity. The mean sensitivity and $\max |s|$ of total porosity to 


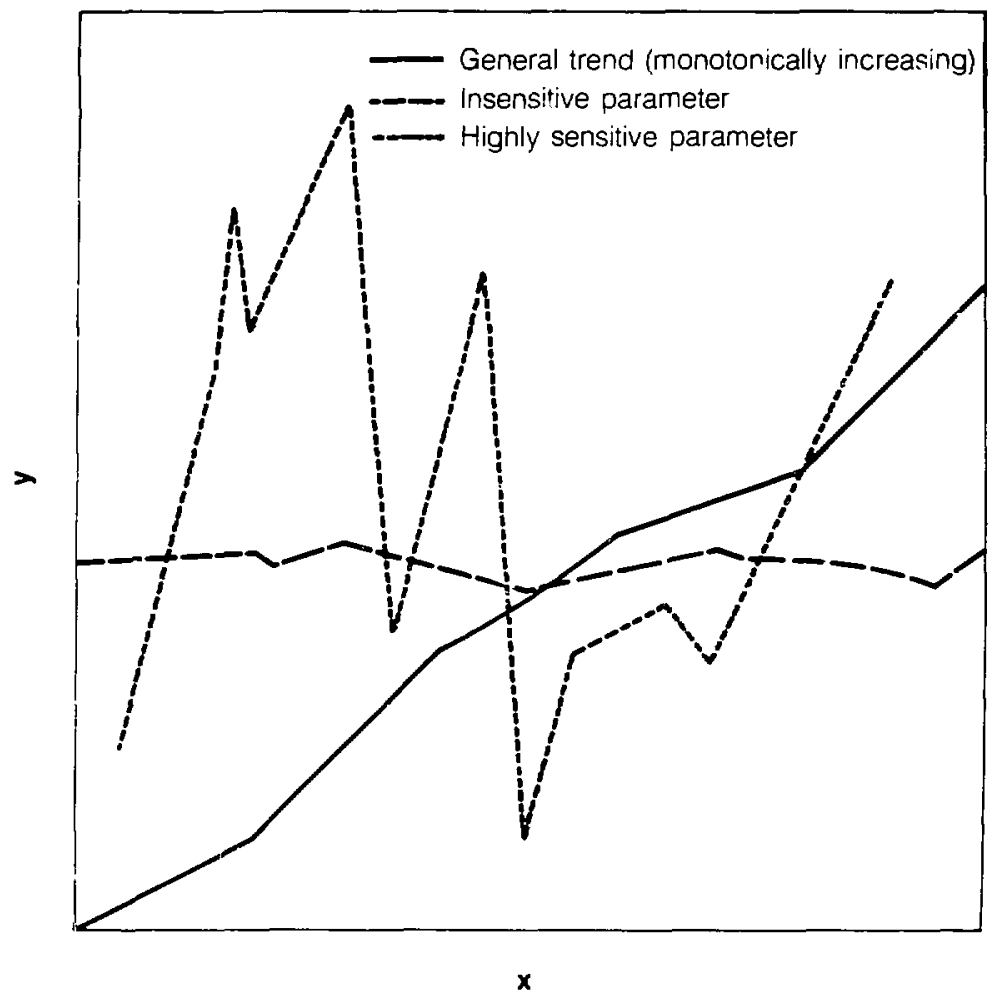

XBL 8310-3299

Figure 6-19 Three General Types of Sensitivity Relationships. 

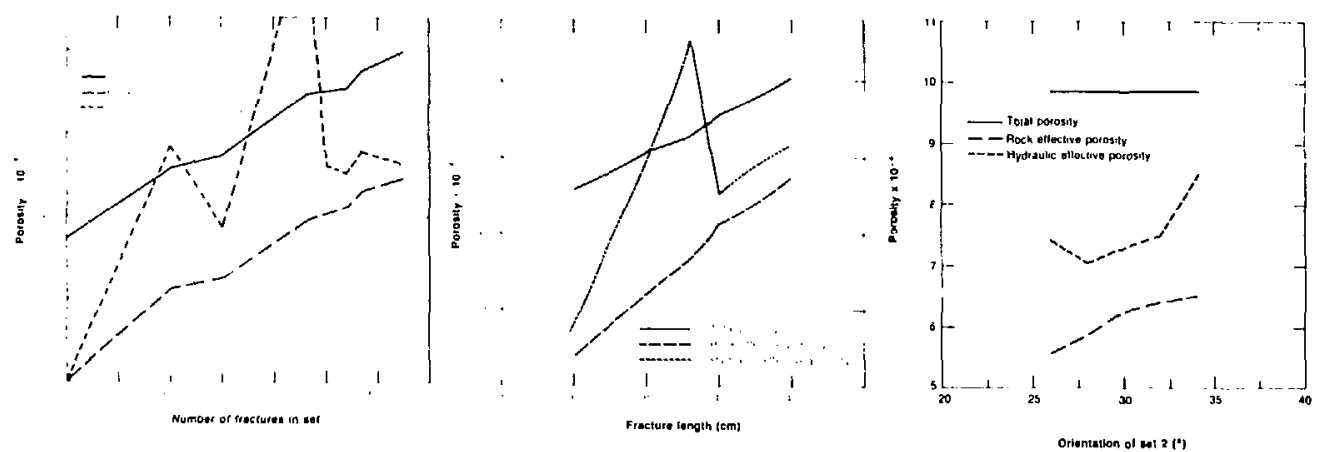

Figure 6-20 Total Porosity, Rock Effective Porosity, and Hydraulic Effective Porosity Versus Number of Fractures in Set, Mean Fracture Length, and Orientation of Set 2. 
mean orientation of set 2 were both zero. The nonsensitivity of $\phi$ to mean orientation of set 2 occurred because the number of fractures in the generation region did not change as the orientation of set 2 was perturbed. However, $\phi_{\mathrm{R}}$ increased as the mean orientation of set 2 increased because connectivity increased. Total porosity and rock effective porosity were most sensitive to fracture length, and least sensitive to the mean orientation of set 2 .

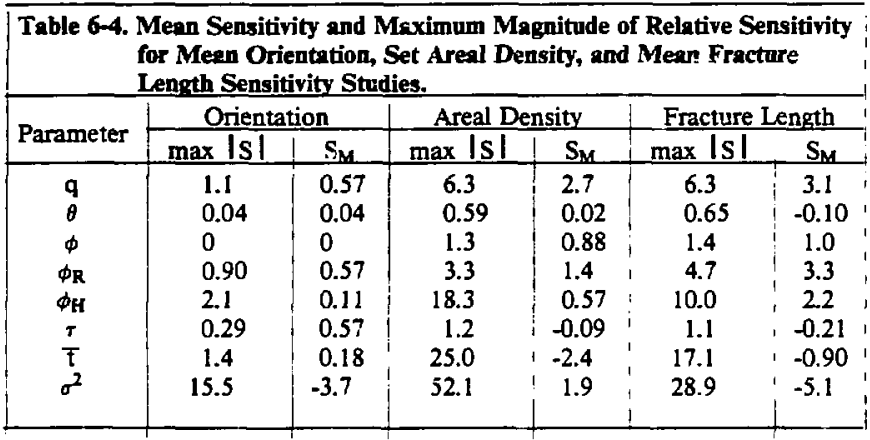

Specific discharge increased as $\mu_{1}, \lambda_{A}$, and $\mu_{0,212}$ increased (Figure 6-21). The $\max |s|$ of $q$ was nearly equal for mean fracture length and set areal density. The $\max |s|$ of $q$ to $\mu_{0, \text { set } 2}$ was almost six times less than the max $|s|$ for $\mu_{1}$ or $\lambda_{\mathrm{A}}$. It was anticipated that the angle of flow would increase as the orientation of set 2 increased. However, the angle of flow was surprisingly insensitive to perturbations in $\mu_{0,0 e t}, \mu_{\mathrm{l}}$, and $\lambda_{\mathrm{A}}$. Thus, $q$ increased as connectivity increased, while $\theta$ was relatively insensitive to connectivi:y.

Rock effective porosity and specific discharge both increased as $\mu_{1}, \mu_{0, s e t}$, and $\lambda_{A}$ increased. Thus, specific discharge and rock effective porosity were each plotted versus connectivity in Figure 6-22 using the results of the three sensitivity studies. The good correlation of both $q$ and $\phi_{R}$ to connectivity shows that both parameiers are fundamentally related to connectivity for this fracture system. Consequently, $q$ and $\phi_{R}$ can be estimated from connectivity when the zeometric parameters of this fracture system are perturbed.

Mechanical transport parameters were generally much more sensitive to $\mu_{0,} \mu_{1}$ and $\lambda_{A}$ than either $\mathrm{q}$ or $\mathrm{PR}_{\mathrm{R}}$. The three plots of mean travel time versus $\mu_{1}, \lambda_{A}$, and $\mu_{0, s e 1}$ in Figure 6-23 

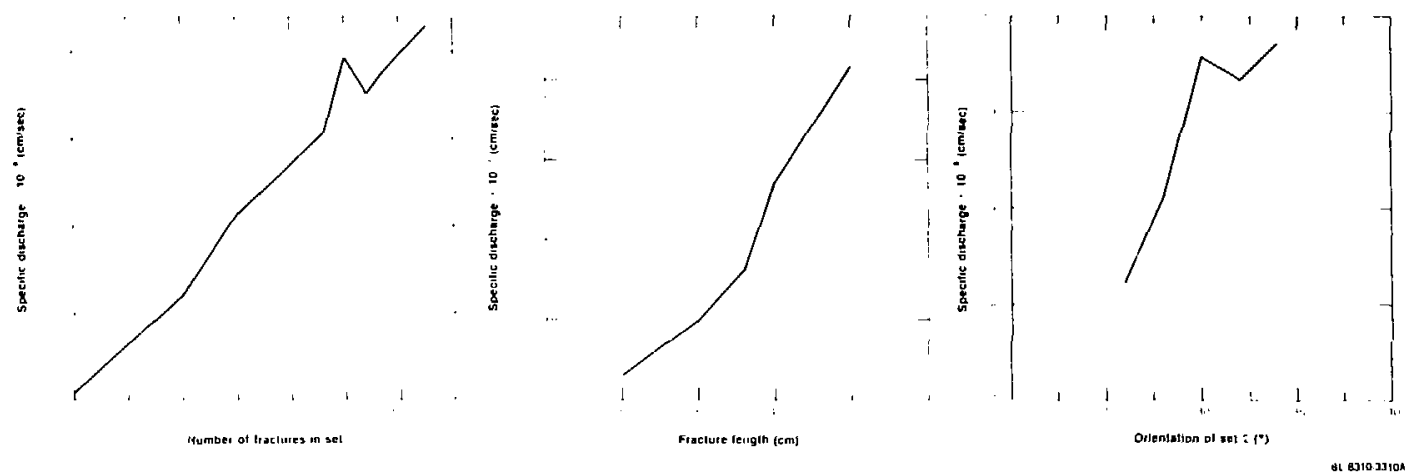

Figure 6-21 Specific Discharge Versus Number of Fractures in Set, Mean Fracture Length, and Orientation of Set 2. 

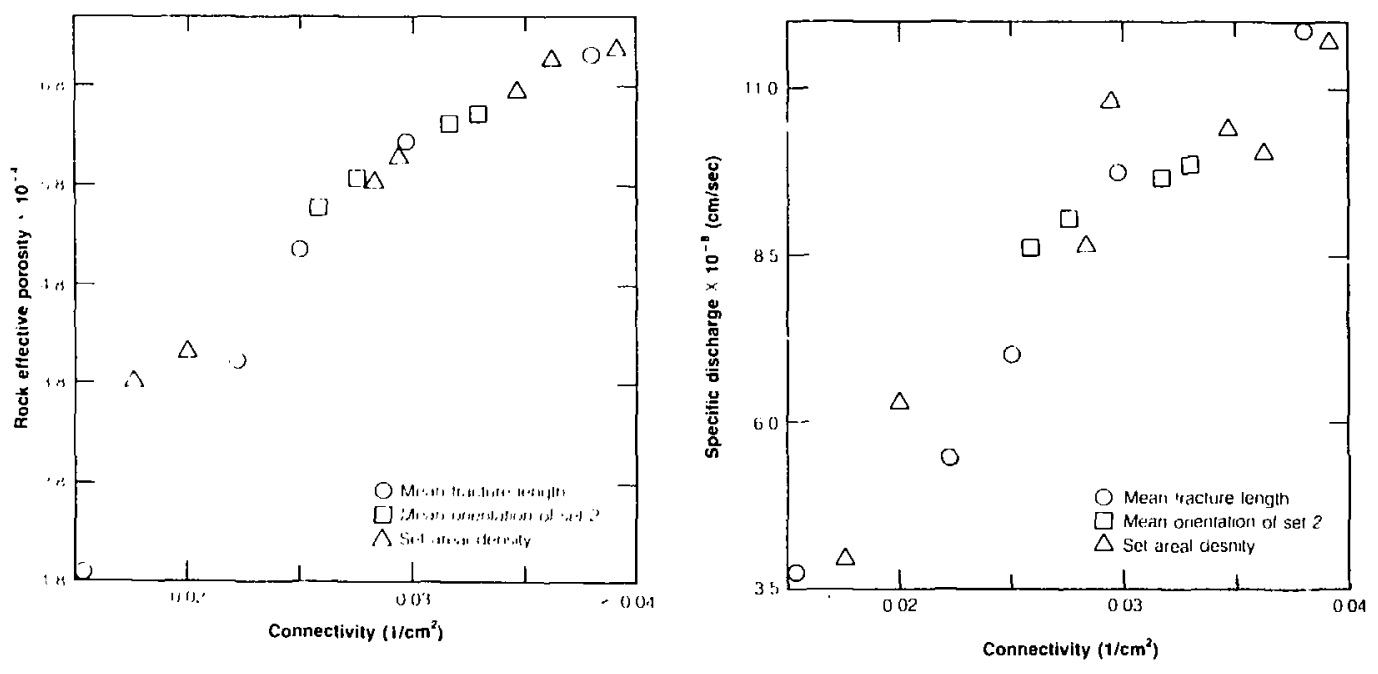

Figure 6-22 Rock Eifective Porosity and Specific Discharge Yersus Connectivity. 
demonstrate the high sensitivity of mechanical transport to each parameter. Three different relationships between $\bar{t}$ and connectivity are observed in this figure, and no general relationship between $\bar{T}$ and any geometric parameter is observed. The high sensitivity of mechanical transport to connectivity is also evident in the variance of the breakthrough curve (Figure 6-24). The variance, $\sigma^{2}$, was the most sensitive mechanical transport parameter, varying by as much as two orders of magnitude in a given sensitivity study. The shape of the $\sigma^{2}$ curve for a paricular mean geometric parameter was similar to the shape of the $\bar{t}$ curve for the same geometric parameter. Thus, $\sigma^{2}$ and $\bar{t}$ showed the same type of relationship to each mean geometric parameter.

As each geometric parameter $\left(\mu_{1}, \mu_{0}, \lambda_{A}\right)$ is perturbed, a different configuration of the ccilductive void region results. Specific discharge was found to relatively insensitive to the configuration of the conductive void region, and fundamentally related to connectivity. However, rnechanical transport was found to be highly sensitive to changes in the configuration of the conductive void region. Thus, mechanical transport parameters cannot be related to connectivity, for these parameters are highly dependent on the configuration of the conductive void Icgion. These results imply the following on the use of double-porosity models. It may be possible to predict fluid flow using a double-porosity model, but it is less likely that such a model can be used to predict transport.

Mechanical transport parameters were generally least sensitive to the mean orientation of set 2 , with the mean fractus $=$ length and the set areal density having about equal sensitivity to each mechanical transport parameter. For example, hydraulic effective porosity was highly sensitive to fracture length and set areal density, and $\phi_{H}$ became larger than $\phi$ in the two sensitivit; studies (Figure 6-22). However, the $\max |S|$ of $\phi_{H}$ to the mean orientation of set 2 was almost four times less than the $\max |S|$ of $\phi_{\mathrm{H}}$ to ejther the mean fracture length or the set areal density.

The connectivity sensitivity study has shown that $\mathrm{q}$ and $\phi_{\mathrm{R}}$ are fundamentally related to connectivity. Specific discharge and rock effective porosity were least sensitive to the mexn 


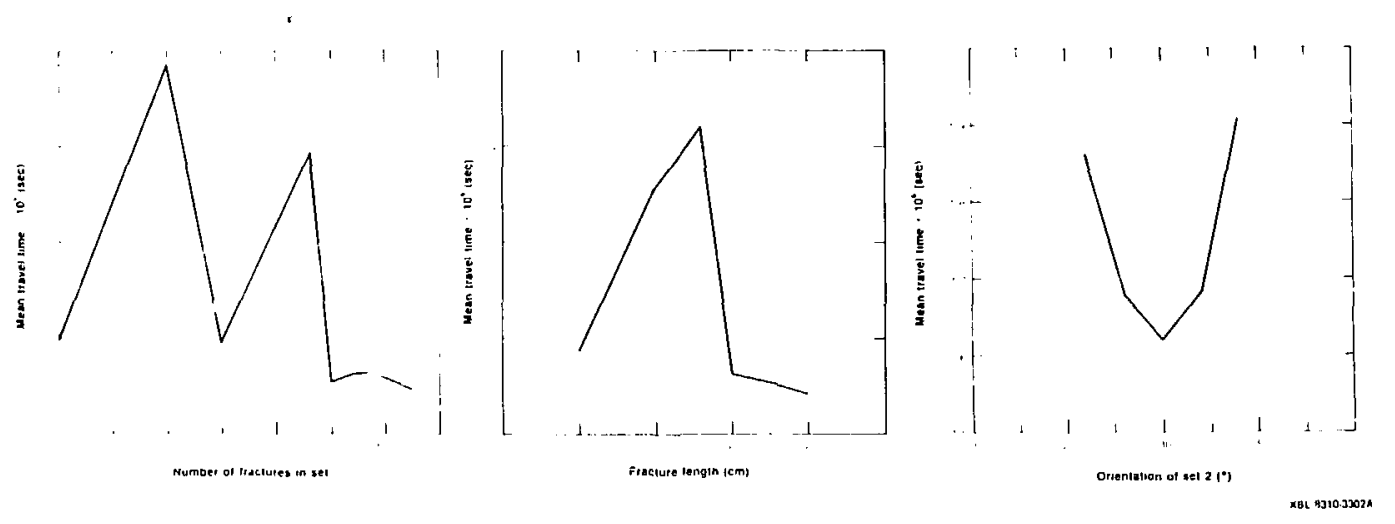

Figure 6-23 Mean Travel Time Versus Number of Fractures in Set, Mean Fracture Length, and Orientation of Set 2. 

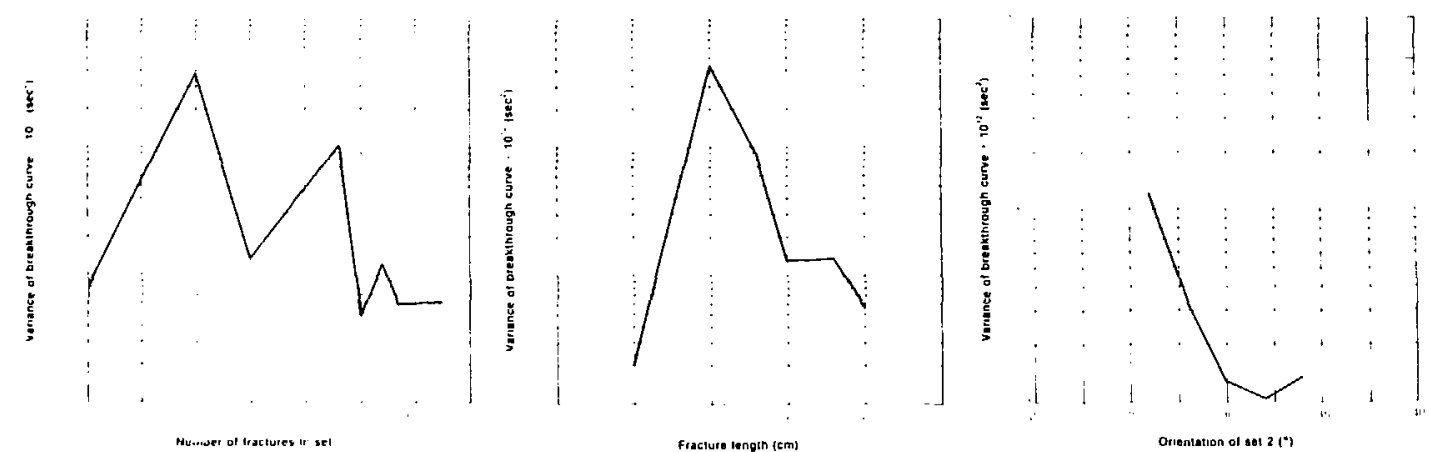

$\stackrel{\dot{N}}{\dot{\infty}}$

Fracture tengith (ctrin)

พBL ด310 $3301 \%$

Figure 6-24 Variance of the Breakthrough Curve Versus Number of Fractures in Set, Mean Fracture Length, and Orientation of Set 2. 
orientation of set 2. Specific discharge exhibited nearly equal sensitivity to the mean fracture length and the set areal density. For this fracture system, angle of flow was relatively insensitive to connectivity.

Mechanical transport parameters were generally much more sensitive to the three connectivity parameters than either $q$ or $\phi_{\mathrm{R}}$. No relationship was found between any of the mechanical transport parameters and connectivity, except for tortuosity. Mechanical transport parameters exhibited the least sensitivity to the mean orientation of set? and $m$ equal sensitivity to the mein fracture length and the set areal density. The most seusitive mechanical transport parameter was $\sigma^{2}$, and the least sensitive was $\tau$.

\subsubsection{Mean Aperture Sensitivity Anslysis}

Mean aperture is the only geometric parameter for which sensitivity can be evaluated. The following relationships were easily formulated for aperture:
a) $\phi \propto \mu_{b}$
b) $\phi_{\mathrm{R}} \propto \mu_{\mathrm{b}}$
c) $\mathbf{q} \propto \mu_{\mathrm{b}}^{3}$
d) $\theta \propto \mu_{0}^{0}$
e) $T \propto \frac{\mathrm{L}}{\frac{\mathrm{q}}{\phi_{\mathrm{R}}}}=\frac{1}{\frac{\mu_{\mathrm{b}}^{3}}{\mu_{\mathrm{b}}}}=\frac{1}{\mu_{\mathrm{b}}^{2}}$
f) $\phi_{\mathrm{H}}=\frac{\mathrm{q}}{\operatorname{VLIN}} \propto \frac{\mu_{\mathrm{b}}^{3}}{\mu_{\mathrm{b}}^{2}}=\mu_{\mathrm{b}}$
g) $r \propto \mu_{b}^{0}$

The relationships listed above were confir ned in the mean aperture sensitivity study which was conducted using two perturbed systeins of aperiure $0.001 \mathrm{~cm}$ and $0.003 \mathrm{~cm}$.

The sensitivity of the variance of the breakthrough curve to aperture was not analytically derived. The mean aperture sensitivity study showed that $\sigma^{2}$ is proportional to $\mu_{b}^{-4}$. The relationship of $\sigma^{2}$ to mean aperture was used to develop the relationships of both $M_{L}$ and $\alpha_{\mathrm{L}}$ to $\mu_{\mathrm{b}}$. Peclet number must be independent of mean aperture from equation 3.20 since the ratio of $\sigma^{2}$ 
to $\vec{t}^{2}$ is proportional to $\mu_{b}^{0}$. The reiationship of $\mathrm{M}_{\mathrm{L}}$ to mean aperture was determined from the Peclet number as:

$$
M_{L}=\operatorname{Pe}\left(V_{L} N\right) L
$$

Since VLIN is, rroportional to $\mu_{b}^{2}, M_{L}$ must also be proportional $\mu_{b}^{2}$. The longitudinal geometric dispersivity is equal to:

$$
\alpha_{L}=\frac{M_{L}}{V L I N}
$$

so $\alpha_{\mathrm{L}}$ must be proportional to $\mu_{\mathrm{b}}^{0}$ since both VLIN and $\mathrm{M}_{\mathrm{L}}$ are proportional to $\mu_{\mathrm{b}}^{2}$. Thus, the mean aperture sensitivity study has shown that $\sigma^{2}, \bar{h}$, and $q$ are highly sensitive to mean aperture, while $T, \theta$, and $\alpha_{L}$ ext sit no sensitivity to mean aperture.

\subsubsection{Sensitivity of Distrihuted Geometric Parameters}

The previous sensitivity studies investigated the sensitivity of mean geometric parameters $(\mu)$. In this section, we shall investigate the effect distributed geometric parameters $(\alpha \neq 0)$ have on mechanical transport and fluid flow. The sensitivity study for a distributed geometric parameter was conducted n the following way. First, a control fracture systern (the same control fracture system used an the previous sensitivity study) was selected. Then, a distributed fracture system was created in which one of the three geometric parameters of length, orientation, or aperture was prob:bilistically simulated. The results for the distributed fracture system were then compared to the control fracture system to determine the effect the distributed geometric parameter had on mechanical transport and fluid flow.

\subsubsection{Distribution of Fracture Orientation}

The first distributed fracture system was created by distributing the fracture orientations in each set according to a normz: distribution. The normal distribution for set 1 had a mean of $0^{\circ}$ and a standard deviaticn $\mathrm{cr} 3^{\circ}$, and the normal distribution for set 2 had a mean of $30^{\circ}$ and a standard deviation of $3^{\circ}$. The distribution of fracture orientations produced a more random fiacture pattern because fiactures wece orienled c. er a wider range of directions. 
Connectivity increased with the randomness of the fracture pattern. As the number of fracture intersections increased, more fracture segments became conductive. The greater conductive void volume caused more fluid to flow through the system, and consequently, $q$ and $\phi_{\mathbb{R}}$ increased for this distributed system.

Deviation in angle of flow was greater for this system than for the control fracture system. The flow field became more nonuniform as paths of fluid particies became more irregular and deviated more from the mean direction of flow. The increased randomness of particle travel paths was reflected by a larger tortuosity. As the paths of particles became longer, the fluid needed more time to flow from side 2 to side 4 . Cor $:=q u e n t l y$, mean travel time increased and the average linear velocity decreased. The increase in $\overrightarrow{\mathbf{q}}$ and decrease in VLIN resulted in an increase in $\phi_{\mathrm{H}}$. This increase in $\phi_{\mathrm{H}}$ was expected since an increase in $\phi_{\mathrm{R}}$ should correspond to an increase in $\phi_{\mathrm{H}}$. The variance of the breakthrough curve also increased because of the increased randomness in particle paths.

Thus, the distribution in fracture orientations created a more random fracture system which caused connectivity to increase. As the fracture pattern became more random, paths of fluid particles became more irregular. The following parameters increasei because of the distribution in fracture orientation: $\phi_{R}, \vec{q}$, DEVA, $\tau, \bar{\tau}$ and $\sigma^{2}$. The average linear velocity decreased for the fracture systen with distributed orientations.

\subsubsection{Distribution of Fracture Aperture}

The second distributed fracture system was created by distributing ihe fracture apertures for each set according to a lognormal distribution with a mean of $0.002 \mathrm{~cm}$ and a standard deviation of $0.0001 \mathrm{~cm}$. The purpose of this study was to show how small aperture fractures created by the distribution control both mechanical transport and fluid flow.

A fluid strean flows through a series of fractures of different apertures in a fracture network. The cubic law states that the flow rate in a fracture is proportional to $b^{3}$. Consequently, the flow rate in a series of fractures is governed by the fracture with the smallest aperture, so that small aperture fractures will negate the large flow capabilities of large aperture fractures. 
Flow rate would only increase if connected pathways of large aperture fractures existed actoss the fracture network. However there is only a small probability of these highly conductive paths developing in a fracture network

The effect of small aperture fractures was demonstrated by the reduction in $\vec{q}$ for this system, as compared with the control fracture system. The smaller flow rate, coupled with the fact that tortuosity did not change, meant that fluid particles took a longer time to travel through the flow region. Consequently, $t$ increased and VLIN decreased. Since both q and VIIN decreased, hydraulic effective porosity did not change.

The distribution of apertures caused a greater variation in the velocities within elements. This resulted in a larger difference in the how rate on opposing sides of the flow region, and a wider distribution in particle travel times in the breakthrough curve. Conseguently, both DEVF and $\sigma^{2}$ increased.

\subsubsection{Distribution of Fracture Leagth}

The third distributed fracture system was created by probabilistically simulating the fracture lengths of both sets according to a lognormal distribution with a mean of $40 \mathrm{~cm}$ and a standard deviation of $4 \mathrm{~cm}$. Connectivity was greatly rediced for this fracture system as a larger portion of the void region became dead-end zones and isolated spaces. The short length fractures caused these nonecnductive void regions to develop in the fractire network. Rock effective porosity and specific discharge decreased as connectivity desreased, and DEVF increased as only a few flow paths were continuous between sides 2 and 4.

\subsubsection{Summary}

The study of distributed geometric parameters has shown that a distribution in fracture orientation caused the fracture network to become more random. The increased randomness in the fracture network caused connectivity, $q, \phi_{\mathrm{R}}$ and $\phi_{\mathrm{H}}$ to increase. When fracture lengths were distributed, connectivity decreased. The short length fractures caused an increase in dead-end zones and isolated void spaces. The reduced connectivity caused both $q$ and $\dot{\phi}_{\mathrm{R}}$ to decrease, 
and DEVF to increase. The distributed aperture study demonstrated that mechanical transport and fluid flow are controlled by the small aperture fractures. The distribution of aperture caused $q$ to decrease and both $\overline{\mathrm{T}}$ and $\sigma^{2}$ to increase. 


\section{CHAPTER 7 \\ INVESTIGATION OF MECHANICAL TRANSPORT AT \\ RESEARCH SITE IN MANITOBA, CANADA}

\subsection{INTRODUCTION}

The Atomic Energy of Canada Limited is conducting hydrologic research at a site located in the province of Manitoba (Figure 7-1). The research program, called the Canadian Nuclear Fuel waste Management Program, has two objectives: 1) to understand fluid flow in a fractured zone in the Lac du Bonnet granitic batholith, and 2) to understand mechanical transport in the same area. The study of fluid flow is being conducted by Long (1984). This chapter presents the study of mechanical transport for this fractured zone.

The computer program described in Chapter 4 was used to study mechanical transport for two verical planes oriented at N-45-W and N-S in a sparsely fractured zone. Each plane intersected the location of the proposed shaft (insert to Figure 7-1). Fracture information must be read into the program to simulate the fracture pattern for the two vertical planes. This sisormation for each piane consists of:

1) The number of fracture sets.

2) $\mu_{0}$ and $\sigma_{0}$ for each set, and the type of probability distribution for fracture orientation.

3) $\mu_{b}$ and $\sigma_{b}$ for each set, and the type of probability distribution for fracture aperture.

4) The areal density for eaclt set.

5) $\mu_{l}$ and $\sigma_{l}$ for each set, and the type of probability distribution for fracture length.

These geometric parameters were determined from hydrogeologic data collected at the site. A detailed discussion of the analysis of the field data is given by Long (1984). The field data conisted of borehole T.V. logs, core logs, and data from well tests for permeability. The borehole T.Y. logs and core logs were used to determine the number of fracture sets. and the mean and 


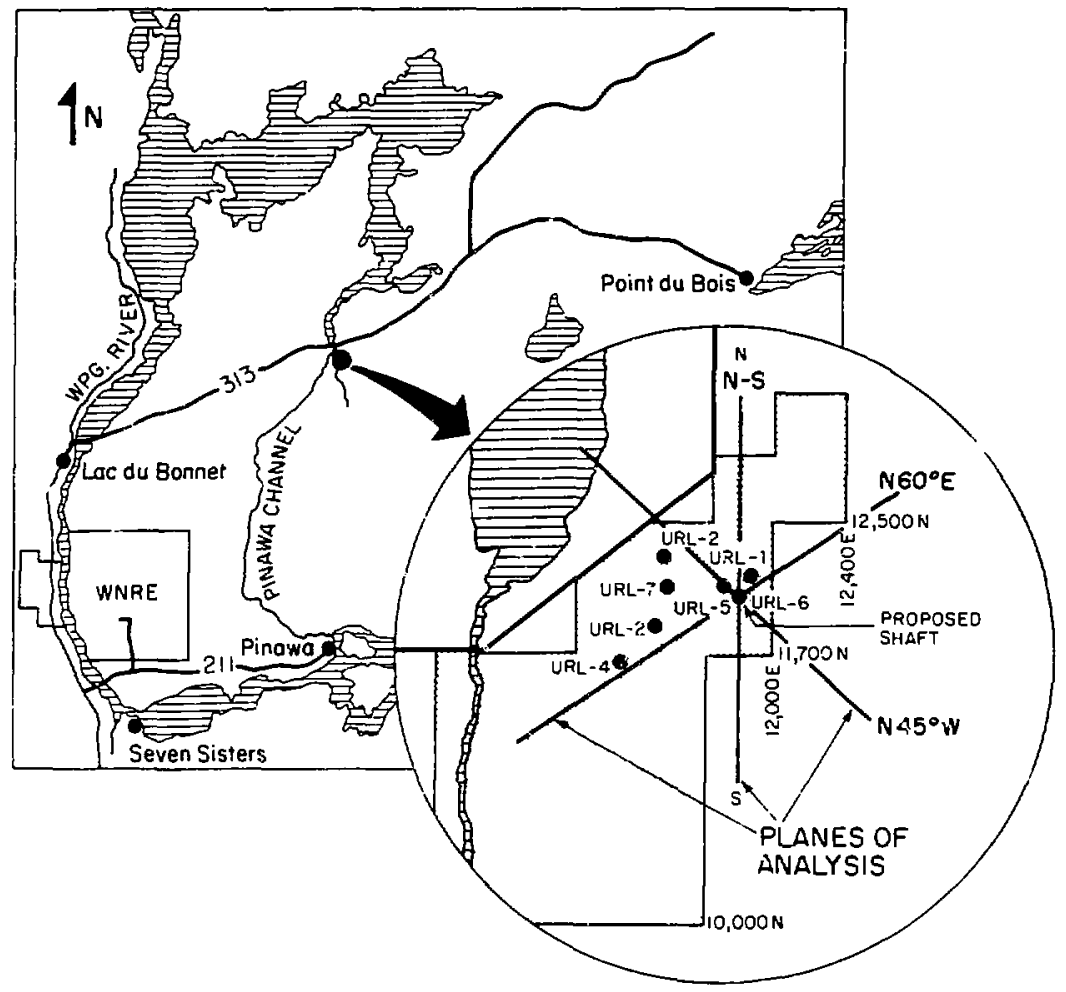

XBLB39-2214

Figure 7-1 Map of Hydrogeolugical Research Site in Manitoba, Canada. 
standard deviation for the frecture orientations of each sel. The fracture orientation statistics in both vertical planes were nearly identical such that only a single set of fracture orientation statistics ( $\mu_{0}$ and $\sigma_{0}$ for each set) was needed for hoth planes. Fracture orientations in each set were assumed to follow the Gaussian distribution.

The well testing data were used to determine the mean and standard deviation for the fracture apertures of each set. The aperture statistics for each set were assumed to be identical. Subsequently, the limited weil testing data did not have to be analyzed separately for each set. The following geometric parameters were determined from the analysis of the field data:

1) The number of sets is 2 .

2) $\mu_{0,2 e 1_{1}}=0^{\circ}, \sigma_{0, \text { set 1 }}=30^{\circ}$

3) $\mu_{0, \text { set } 2}=90^{\circ}, \quad \sigma_{0, \text { set } 2}=35^{\circ}$

4) $\mu_{\mathrm{b}}=0.00005 \mathrm{~m}, \quad \sigma_{\mathrm{b}}=0.00005 \mathrm{~m}$

The set areal density, mean fracture length, and standard deviation of fracture length could not be determined from the borehole data. Thus, a length-density sensitivity analysis was conducted to investigate how length and density influences mechanical transport. In this analysis, each fracture set was assumed to have the same areal density and the same fracture length statistics, with the standard deviation of length assumed equal to $\mu_{\text {! }}$. Fracture lengths were assurned to be lognormal!y distributed. The following relationship was used to relate mean fracture length and set areal density (Long, 1984):

$$
-\frac{\lambda_{1}}{\mu_{\text {cos }}}=L D=\mu_{1} \lambda_{A}
$$

The linear density, $\lambda_{!}$, is the number of fractures intersecting a unit length of scanline. Linear density was computed in this study by counting the number of open fractures intersecting each borehole, and then dividing by the total length of the borcholes. The mean of $\cos \xi$ is a correction factor used to account for the fact that fractures that are perpendicular to thr scanline have a greater probability of intersecting the scanline than fractures that are parallel to this line. From $\lambda_{1}$ and $\mu_{\text {coss }}$, the constant lengih-density parameter LD was computed to be $0.1 \mathrm{~m}^{-1}$. As 
an example of the use of this length-density relationship, a fracture set with a mean iracture length of 1 , would have the following length statisties and set areal density:

$$
\begin{aligned}
& \mu_{1}=l_{2}, \sigma_{1}=l_{1} \\
& \lambda_{A}=0.1 / l_{1}
\end{aligned}
$$

Two series of length-density sensitivity studies were conducted: 1) the constant aperture series and; 2) the distributed aperture series. The constant aperture series was conducted to investigate mechanical transport aused strictly by the configuration of the fracture pattern, ignoring the heterogeneity that results by distributing apertures. Two studies were conducted in the distributed aperture series because the aperture distribution has a great influence on mechanical transport: 1)study with $\mu_{\mathrm{b}}$ and $\sigma_{\mathrm{b}}$ both equal to $0.00005 \mathrm{~m}$ and, 2) study with $\sigma_{\mathrm{b}}$ equal to $0.3 \mu_{b}$. The mean $b^{3}$ (cubic law) was held constant in the two series so tnat the expected permeability would be the same in the two series. In the first distributed aperture study, fracture apertures were lognormally distributed in both sets, with a mean of 0.c000s $\mathrm{m}$ and a standard deviation of $0.00005 \mathrm{~m}$. The mean $\mathrm{b}^{3}$ for this probability distribution is $1 \times 10^{-12} \mathrm{~m}^{3}$. Consequently, this mean $b^{3}$ was maintained in both series such that an aperture of $0.0001 \mathrm{~m}$ was used in the constant aperture series.

\subsection{CONSTANT APERTURE LENGTH-DENSTTY SERIES}

Fracture systems with different fracture lengths and set areal densities were created using equation 7.1:

$$
L D=0.1=\lambda_{A} \mu !
$$

Each fracture system consisted of two fracture sets with the same $\lambda_{\mathrm{A}}$. The fracture orientations for set 1 were distributed using a normal distribution with a mean of $0^{\circ}$ and a standard deviation of $30^{\circ}$. The fracture orientations for set 2 were distributed using a nermal distribution with a mean of $90^{\circ}$ and a standard oeviation of $35^{\circ}$. All fractures had an aperture of $0.0001 \mathrm{~m}$, and frac:ure lengths were distributed using a lognormal distribution with a mean of $\mu_{1}$ and a standard deviation also equal to $\mu_{\text {f }}$. Each set had an areal density of $0.1 / \mu_{\mathrm{f}}$. 
The width of the square generation region used in each length study was twenty times larger than $\mu_{1}$. Square flow regions of width ten times $\mu_{1}$, oriented at every $15^{\circ}$, were created for each fracture system to study mechanical transport. The hydraulic gradient on sides 1 and 3 for all flow regions was set at 0.01 .

The first two fracture systems were created with $\mu_{1}$ equal to $10 \mathrm{~m}\left(\lambda_{\mathrm{A}}=0.01 \mathrm{~m}^{-2}\right)$ and $\mu_{1}$ equal to $20 \mathrm{~m}\left(\lambda_{\mathrm{A}}=0.005 \mathrm{~m}^{-2}\right)$. The fracture pattern in the generation region for the fracture systern with $\mu_{1}$ of $10 \mathrm{~m}$ is shown in Figure 7-2. The fracture pattern and network of connected fracture segments for the flow region oriented at $0^{\circ}$ for this fracture system are both shown in Figure 7-3. Both fracture systems were so sparse that a zone of continuous flow did not develop between sides 2 and 4 . Thus, neither system behaved like equivalent porous medium. In a porous medium, a continuous zone of flow exists in a square flow region unless the angle of flow is greater than $45^{\circ}$. The geometric parameters for the two systems indicate that both systems should be fairly isotropic, such that the angle of flow will not exceed $45^{\circ}$. No study of mechanical transport was conducted for the two systems because of the absence of the continuous zone of flow between sides 2 and 4 in any flow region. The fracture system with $\mu_{1}$ of $30 \mathrm{~m}$ was the first system studied wh ch had a cortinuous zone of flow between sides 2 and 4 . In addition to the fracture system with $\mu_{1}$ of $30 \mathrm{~m}$, two other discontinuous systems with $\mu_{1}$ of 35 $\mathrm{m}$ and $50 \mathrm{~m}$ were studied. For the fracture system with $\mu_{1}$ of $50 \mathrm{~m}$, the ividth of the flow reg:on was reduced to seven times $\mu_{1}$ because of compuici storage. The fracture pattern in the generation region tor the fractu-e system with $\mu_{1}$ of $35 \mathrm{~m}$ is shown in Figure 7-4. The fracture pattern and network of connected fracture segments for the flow region oriented at $0^{\circ}$ for this system are shown in Figure 7-5

A continuous fracture jystem $\left(\mu_{i}=\infty\right)$ was also created to study mechanical transport. This system was created in the following way. For each set, a scanline was drawn perpendicular to the mean orieritation of the set across :he entire generation region. The square generation region had a width of $650 \mathrm{~m}$. This scanline passed through the center of the generation region. The frasiste centers of the set were then randomly located on this scanline. The number of 


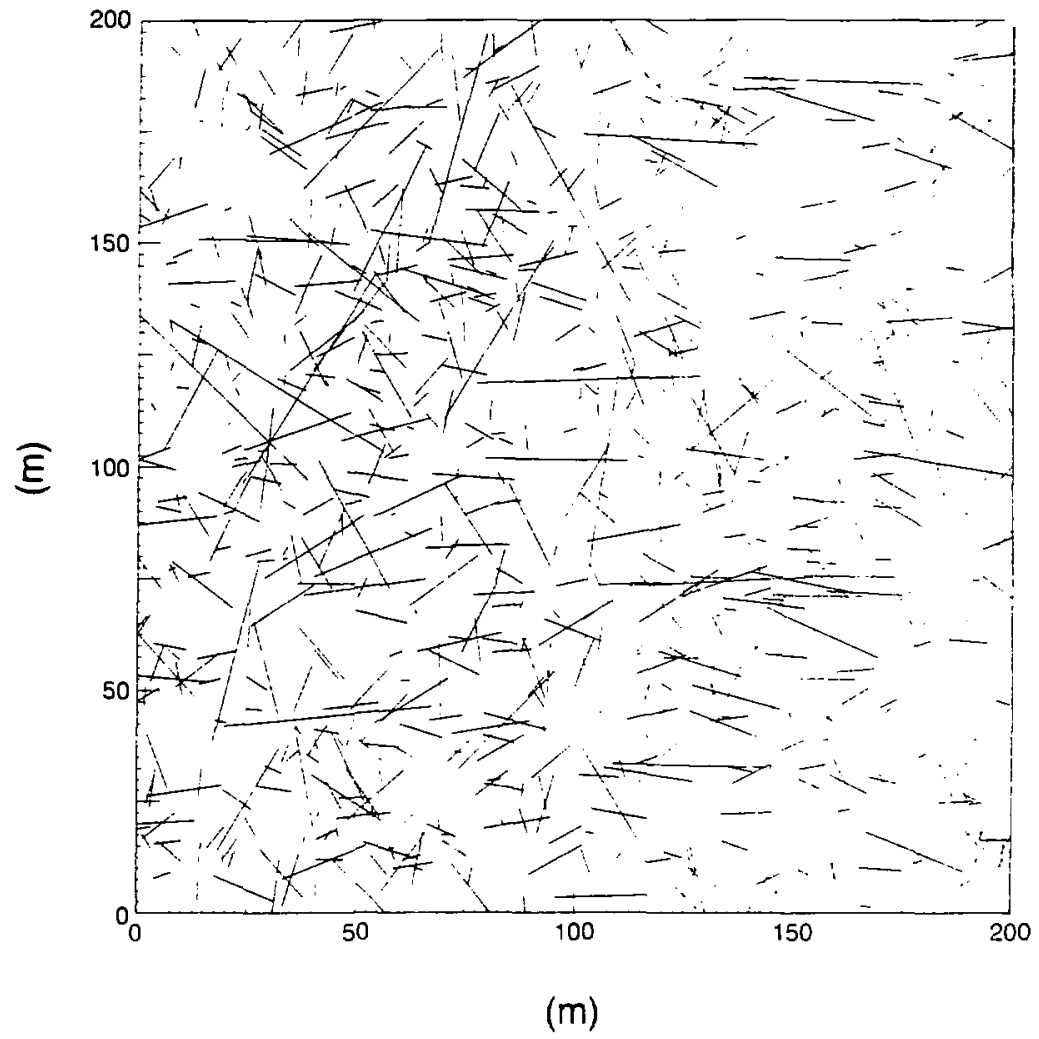

XEL $84 !-392$

Figure 7.2 Fracture Network in the Generation Region for Discontinuous System With Mean Fracture Length of $10 \mathrm{~m}$. 
(a)

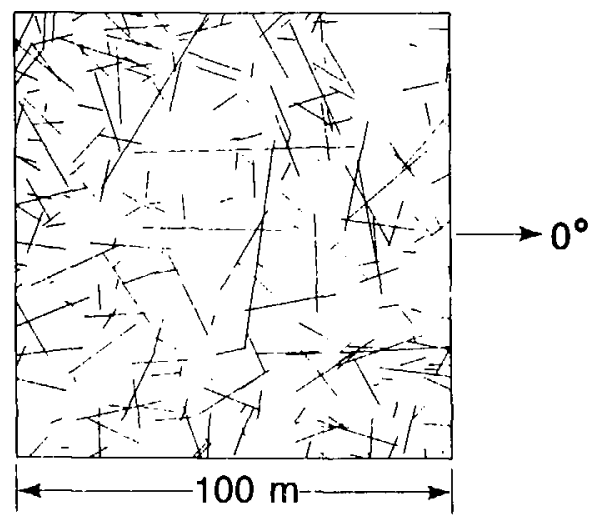

(b)

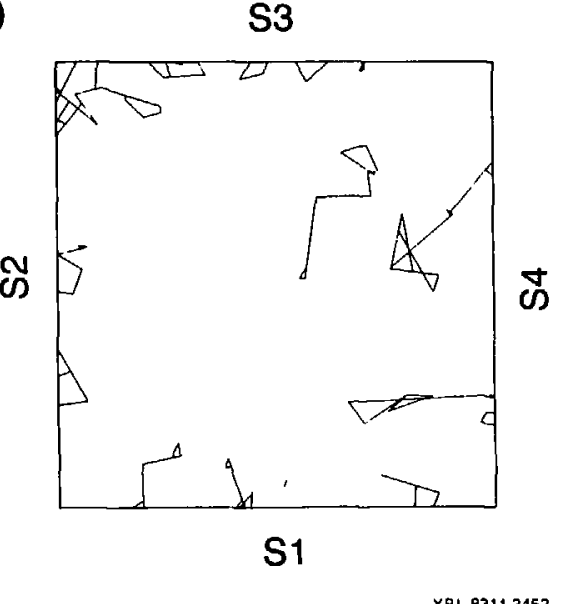

Figure 7-3 Network of a) Fractures and b) Connected Fracture Segments in the Flow Region Oriented at $0^{\circ}$ for Discontinuous System With Mean Fracture Length of $10 \mathrm{~m}$. 


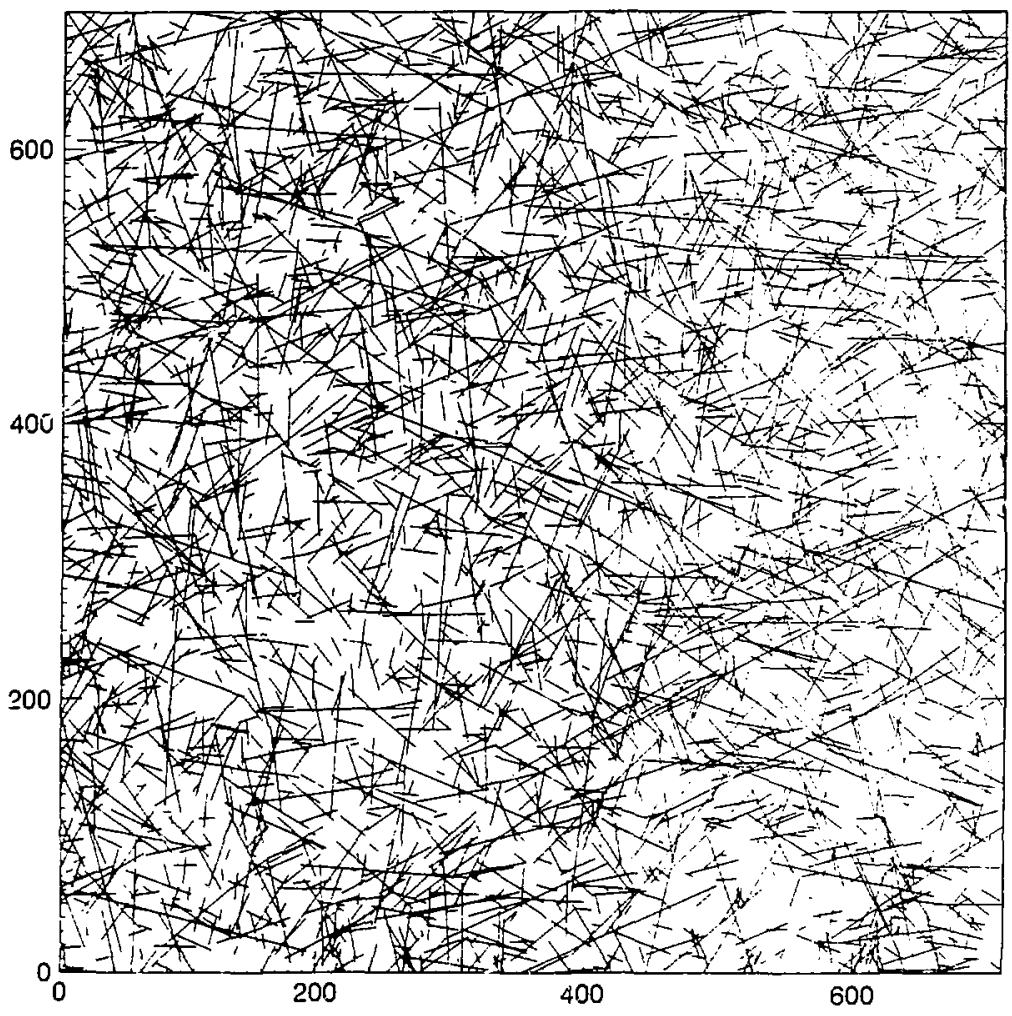

(m)

XBL $641-393$

Figure 7-4 Fracture Network in the Generation Region for Discontinuous System With Mean Fracture Length of $35 \mathrm{~m}$. 
(a)

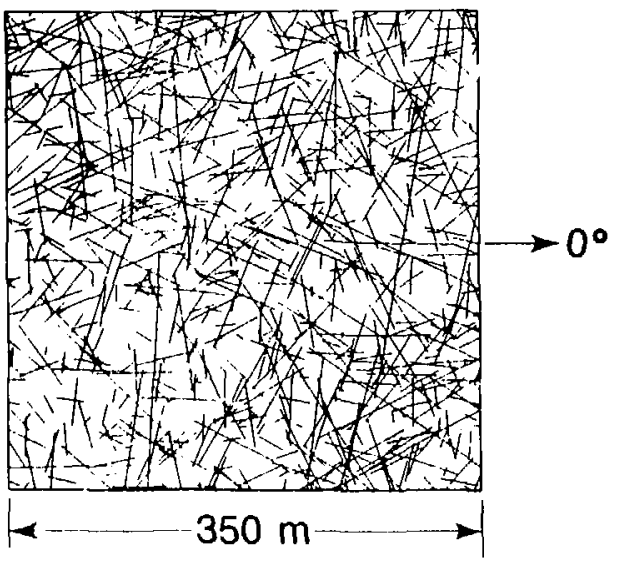

(b) S3

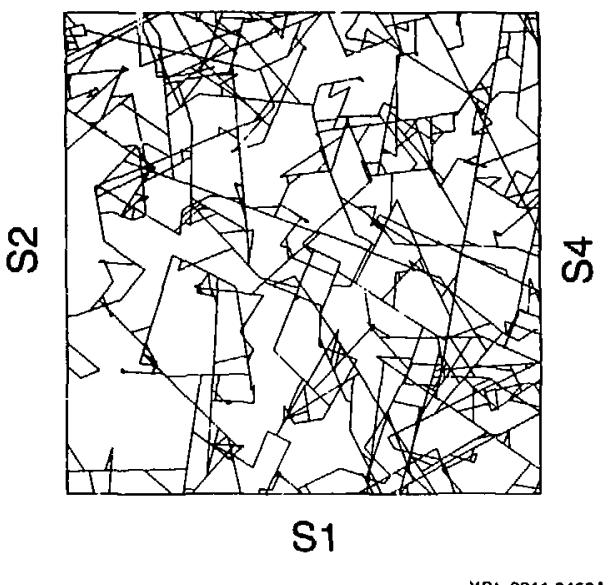

Figure 7-5 Network of a) Fractures and b) Connected Fracture Segments in the Flow Region Oriented at $0^{\circ}$ for Discontinuous System With Mean Fracture Length of $35 \mathrm{~m}$. 
fracture centers located on the scanline was equal to (LD) $\left(\mu_{\text {cos }}\right)$ LS, where LS is the length of the scanline. Next, fracture orientations and apertures were distributed from Gaussian and lognormal distributions, respectively. Each fracture in the set was assigned a length much larger than the width of the generation region. After the completion of the fracture network, square flow regions of width $250 \mathrm{~m}$, oriented at every $15^{\circ}$, were used to study mechanical transport.

Figure 7-6 shows the tortuosity versus direction of flow for the continuous fracture system and the three discontinuous systems. In all four cases, tortuosity is stable with direction. This indicates that these fracture systems are faity isotropic. The permeability study conducted by Long (i984) ajso found that the four systems are fairly isotropic. Mean tortucsity does not vary significantly for the three discontinuous systems. The mean tortuosities were $1.367,1.403$, and 1.368 for the discontinuous systems with $\mu_{1}$ of $30 \mathrm{~m} .35 \mathrm{~m}$, and $50 \mathrm{~m}$, respectively. The [. ean tortuosity for the continuous system is $1.26 \mathrm{l}$. The fractures in the continuous system span across the entire flow regicn. Consequently, fluid flows in a more direct route across the flow region in the continuous fracture system than in any of the discontinuous systems. This caused tortuosity to be lower for the continuous system.

Hydraulic effective porosity is shown piotted against direction of flow in Figure 7-7. No directional dependence in $\phi_{\mathrm{H}}$ is apparent for any of the four fracture systems. Since hydraulic effective porosity is relatively constant in all directions for the four systems. each system behaves like an equivalent porous medium tor transpor. The mean $\phi_{H}$ are 0.0000133 , 0.0000141 , and 0.0000147 for the three disccntinuous systems with $\mu_{1}$ of $30 \mathrm{~m}, 35 \mathrm{~m}$, and $50 \mathrm{~m}$, respectively. Rock effective porosity is constant at 0.000013 for the three discontinuous sys. tems. Consequentiy, $\phi_{H}$ is greater than $\phi_{R}$, and $\phi_{H}$ increases with $\mu_{1}$ in the discontinuous systems. The mean $\phi_{H}$ is 0.0000213 for the continuous fracture system. This value is slightly iess than the total porosity of 0.0000235 . Hydraulic effective porosity is misch greater for the continuuus system than for the discontinuols system because no dead-ead fracture segments exist in the continuous system. The void volurs: is totally cornected for continuous systems. The total porosity for the three discontinuous systems of 0.0000195 is only slightly less than $\varnothing$ for 
(a)

240

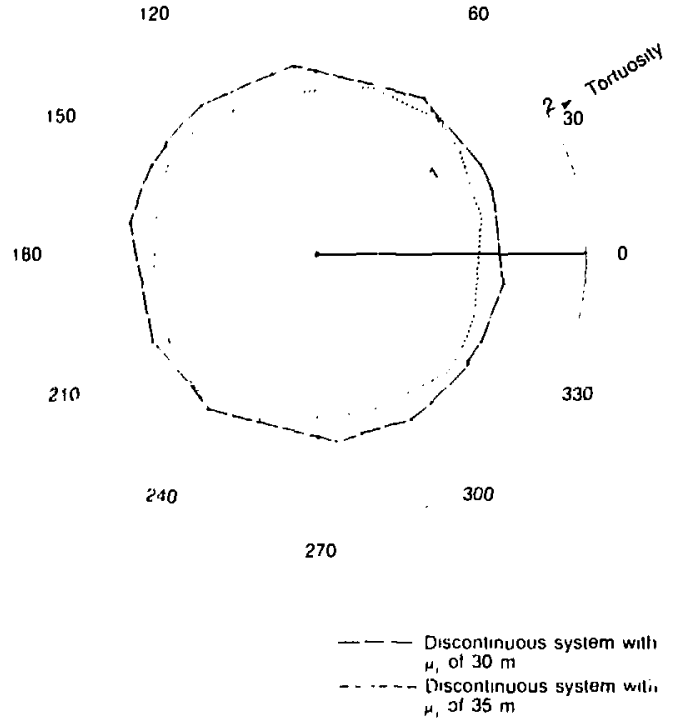

(b)
90

Figure 7.6 Polar Plot: of Tortuosity for a) Systems With Mean Fracture Lengths of 30 and $35 \mathrm{~m}$ and b) System Witil Mean Fracture Length of $50 \mathrm{~m}$ and the Continuous Fracture System. 
120

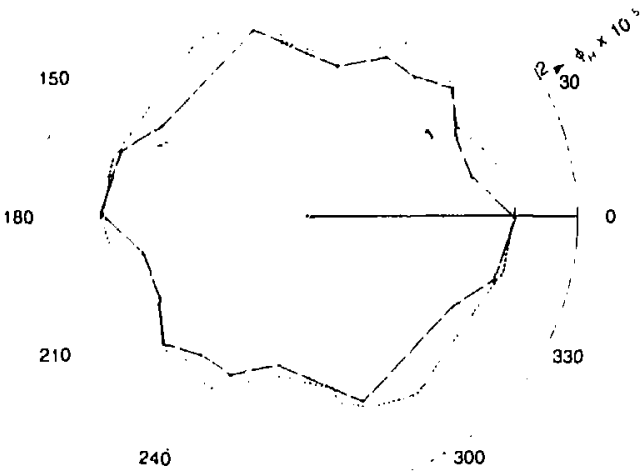

270 (b)

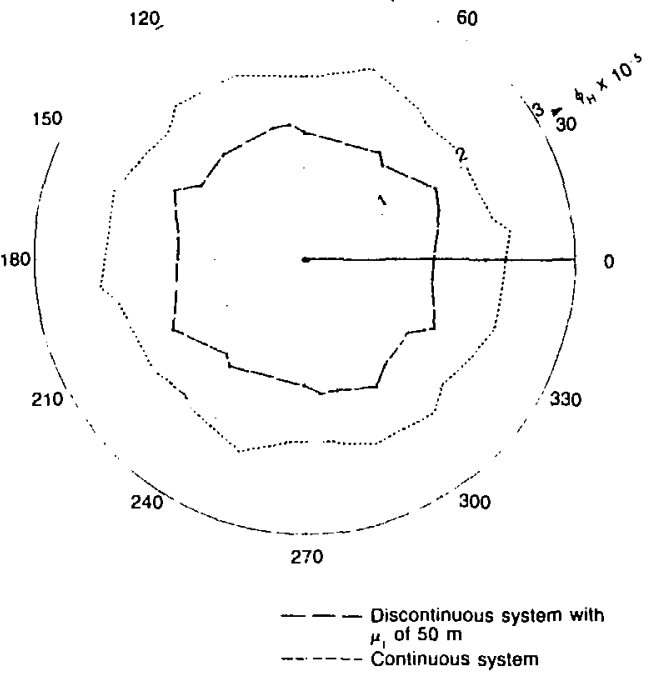

Figure 7-7 Polar Plots of Hydraulic Effective Porosity for a) Systems With Mean Fracture Lengths of 30 and $35 \mathrm{~m}$ and b) System With Mean Fracture Length of $50 \mathrm{~m}$ and the Continuous Fracture System. 
the continuous system. However, ouly 67 percent of this void volume is conductive.

Figures 7-8 and 7-9 show the polar plots of longitudinal geometric dispersivity for the four s. stems. The discontinuous system with $\mu_{1}$ of $30 \mathrm{~m}$ has a maximum $\alpha_{\mathrm{L}}$ of $99 \mathrm{~m}$ at direction of flow 78. The next largest $\alpha_{\mathrm{L}}$ occurs in a direction which is r. early perpendicular to the direction of maximum $\alpha_{\mathrm{L}}$. The mean $\alpha_{\mathrm{L}}$ for this system is $61.1 \mathrm{~m}$, and the standard deviation in $\alpha_{\mathrm{L}}$ is $18.4 \mathrm{~m}$. The polar plot of $\alpha_{\mathrm{L}}$ is very different for the discontinuous system with $\mu_{1}$ of $35 \mathrm{~m}$. Longitudinal geometric dispersivity shows large directional variations, as the $\alpha_{\mathrm{L}}$ curve is very jagged. The ratio of $\alpha_{L \max }$ to $\alpha_{L, \min }$ is four. The two largest values of $\alpha_{\mathrm{L}}$ occur in directions that are nearly orthogonal te each other. Th: mean $\alpha_{\mathrm{L}}$ for this system is $63.5 \mathrm{~m}$, and the standard deviation in $\alpha_{\mathrm{L}}$ is $36.0 \mathrm{~m}$. The directional variation in $\alpha_{\mathrm{L}}$ is less for the discontinuous sysien with $\mu_{l}$ of $50 \mathrm{~m}$ than for the discontinuous system with $\mu_{l}$ of $35 \mathrm{~m}$. The ratio of $\alpha_{L, m a x}$ to $x_{L, \min }$ is three, and the standard deviation in $\alpha_{L}$ is $20.3 \mathrm{~m}$. The mean $c_{L}$ for this system is 66.4 m. For the continuous system, $\alpha_{L}$ shows a strong directional defondence. The ratio of $\alpha_{L, \max }$ to $\alpha_{\mathrm{Lmin}}$ is nine. The two directions of maxima $\alpha_{\mathrm{L}}$ are $0^{\circ}$ and $90^{\circ}$. These two directions are the mean orientations for the two sets. The mean $\alpha_{\mathrm{L}}$ is $30.3 \mathrm{~m}$ for the continuous system.

The following conclusions can be made about the $\alpha_{\mathrm{L}}$ study. The direction of maximurr. $\alpha_{\mathrm{L}}$ for each system is located between $80^{\circ}$ and $170^{\circ}$. The next largest $\alpha_{\mathrm{L}}$ for each system is obtained in a direction that is nearly perpendicular to the direction of maximum $\alpha_{L}$. The polar plots of $\alpha_{L}$ are very different for eacn system, as $\alpha_{L}$ exhibits a unique directional variation in each system. However, the mean-directional longitudinal geometric dispersivities for the three discontinuous systems are nearly the same so that mean $\alpha_{\mathrm{L}}$ is independent of mean fracture length.

The highly directional nature of $\alpha_{L}$ was not expected in this study. Theoretically, $\alpha_{L}$ should be constant in all directions in an isotropic porous medium. Therefore, we expected the polar plots of $\alpha_{\mathrm{L}}$ to be nearly circular. To see if $\alpha_{\mathrm{L}}$ approaches a directionally stable value, four additional realizations were studied for the discontinuous system with $\mu_{1}$ of $s 0 \mathrm{~m}$. The width of flow region used in these realizations was $330 \mathrm{~m}$. 
(a)

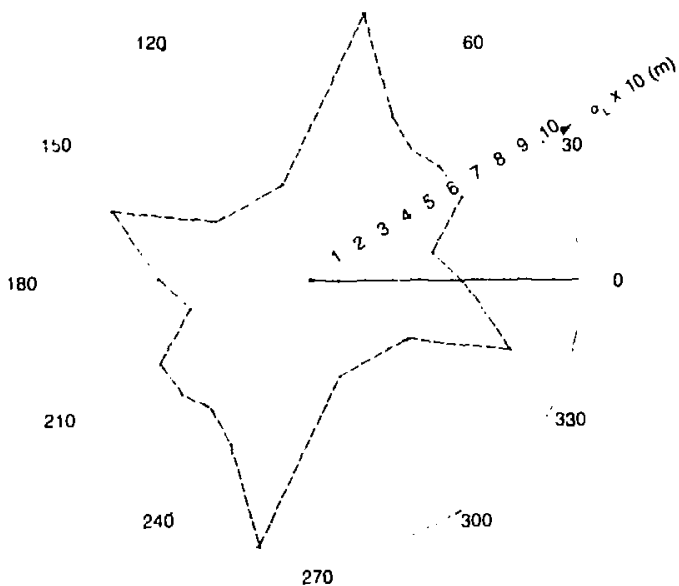

(b)

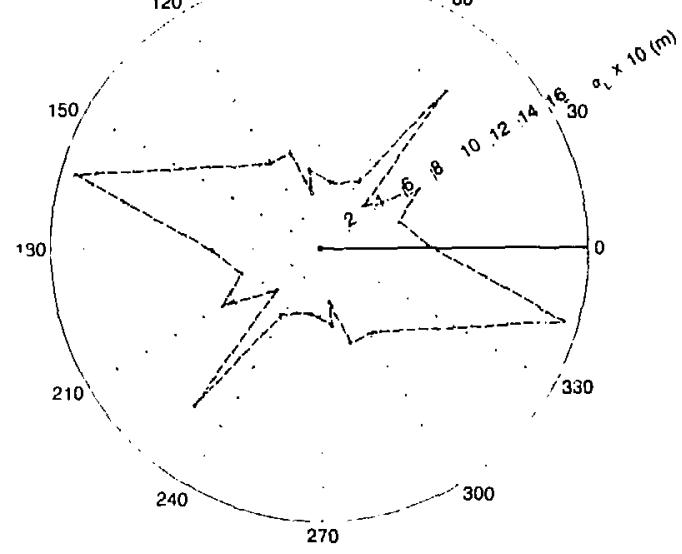

(B) H4:-344

Figure 7-8 Polar Plots of Longitudinal Geometric Dispersivity for Systems With Mean Fracture Lengths of a) $30 \mathrm{~m}$ and b) $35 \mathrm{~m}$. 
(a)

120

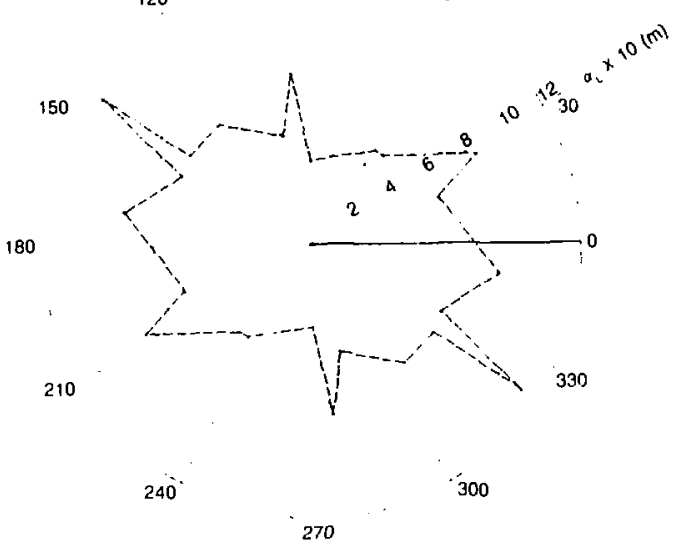

(b)

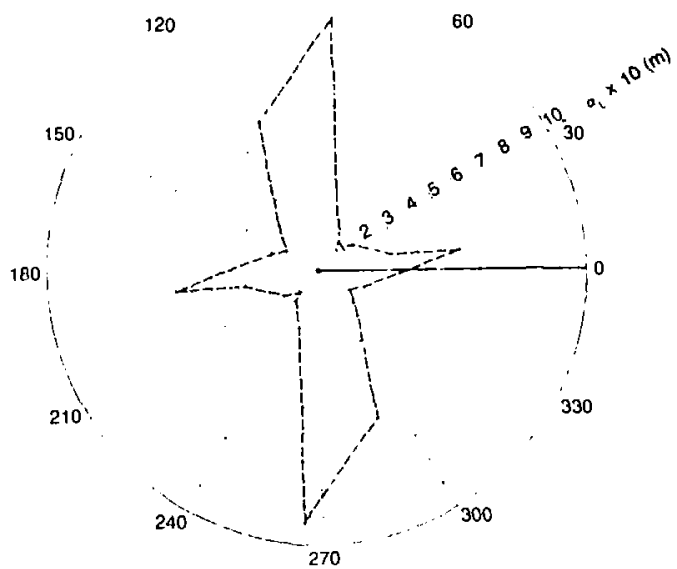

Figure 7-9 Polar Plots of Longitudinal Geometric Dispersivity for a) System W'ith Mean Fracture Length of $50 \mathrm{~m}$ and $\mathrm{b}$ ) the Continuous Fracture Systen. 
All five realizations showed large directional variations in $\alpha_{\mathrm{L}}$. Figure $7-10$ is a polar plot of the mean $\alpha_{L}$ for the five realizations. Longitudinal geometric dispersivity is directionally stable between $20^{\circ}$ to $80^{\circ}$. As direction of flow varies from $80^{\circ}$ to $130^{\circ}$, $\alpha_{\mathrm{L}}$ increases rapidly to its maxirrum value of $110 \mathrm{~m}$. The ratio of $\alpha_{\mathrm{L}, \max }$ to $\alpha_{\mathrm{L}, \min }$ is 2.8 . Thus, longitudinal geometric dispersivity does not appear to be approaching a directionally stable value, as longitudinal geometric dispersivity is stable only between $20^{\circ}$ to $80^{\circ}$. This was not the first fairly isotropic medium which had a directionally dependent $\alpha_{\mathrm{L}}$. It will be recalled from section 5.3 that $\alpha_{\mathrm{L}}$ was directionally dependent for the continuous fracture system of two orthogonal sets with constant apertures which behaved like an equivalent isotropic medium for fluid flow. The mean $\alpha_{\mathrm{L}}$ for the five realizations is $65.7 \mathrm{~m}$. This value is about equal to the mean longitucinal geometric dispersivities measured earlier for the three discontinuous systems. We expect that if $\alpha_{\mathrm{L}}$ were to converge to a directionally stable value, this value would be about $65 \mathrm{~m}$.

The four systems behaved like equivalent porous media for transport because $\phi_{\mathbf{H}}$ was directionally stable in each system. A study was made to see what happens to $\phi_{H}$ when the fracture system does not behave like an equivalent porous medium. The fracture system in the last realization of the previous longitudinal geometric dispersivity study was used for this study. Square flow' regions of widths $60 \mathrm{~m}, 175 \mathrm{~m}$, and $330 \mathrm{~m}$ were oriented at every $15^{\circ}$, beginning at $0^{\circ}$ within the generation region. For each flow region, the computer program was used to calculate hydraulic effective porosity.

Figure 7.11 is a polar plot of $\phi_{H}$ measured using flow regions of width $330 \mathrm{~m}$. Hydraulic effective porosity is nearly the sime in all directions. The mean $\phi_{H}$ is 0.0000148 and the standard deviation in $\phi_{\mathbf{H}}$ is $1.28 \times 10^{-6}$. Figure $7-12$ shows the polar glot of hydraulic effective porosity measu:ed using flow regions of width $175 \mathrm{~m}$. The mean hydraulic effective porosity is 0.0000149 . Thus, the mean hydraulic effective porosity did not change as the size was lowered. The standard deviation in $\phi_{\mathrm{H}}$ increased slightly to $1.35 \times 10^{-6}$. The polar plot of $\phi_{\mathrm{H}}$ is still approximately a circle, and we can conclude that on this scale the system behaves like a continuum for transport. Figure 7-13 shows the polar plot of $\phi_{\mathrm{H}}$ measured using flow regions of 


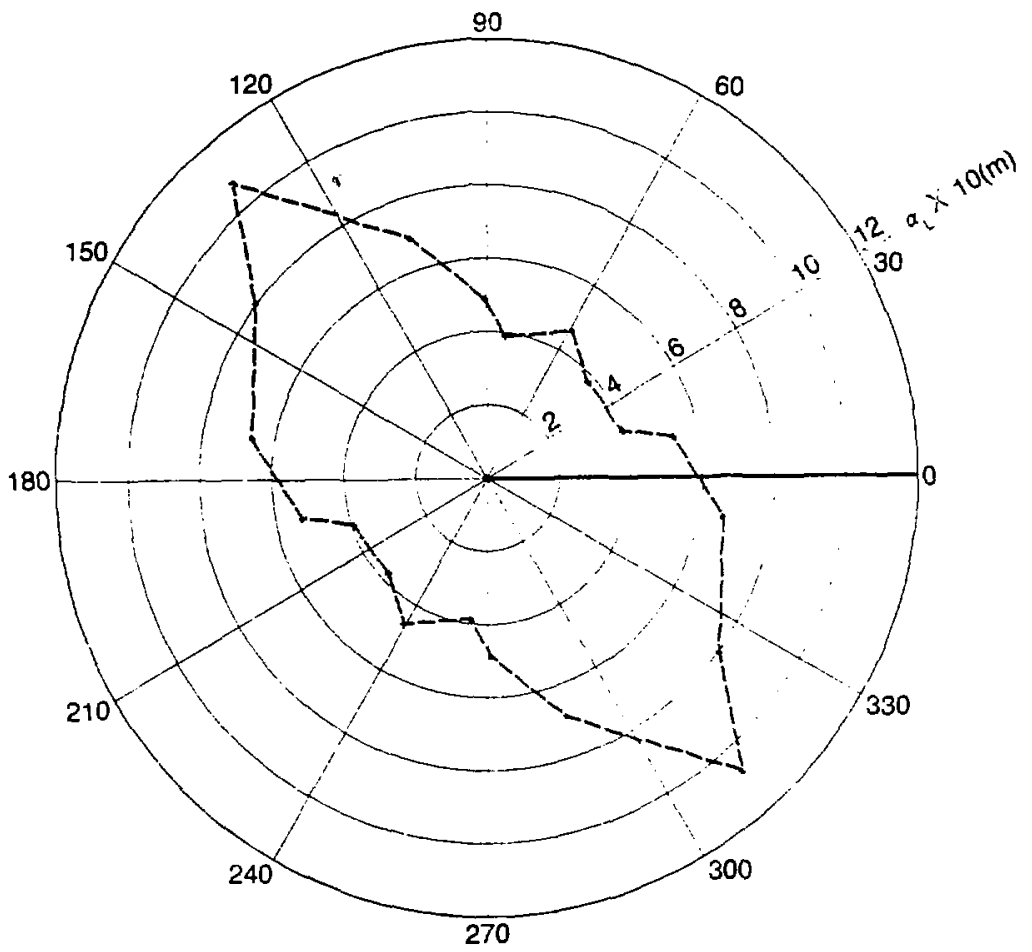

XEL 8401-6799

Figure 7-10 Polar Plot of Mean Longitudinal Geometric Dispersivity for Five Realizations of the System With Mean Fracture Length of $50 \mathrm{~m}$. 


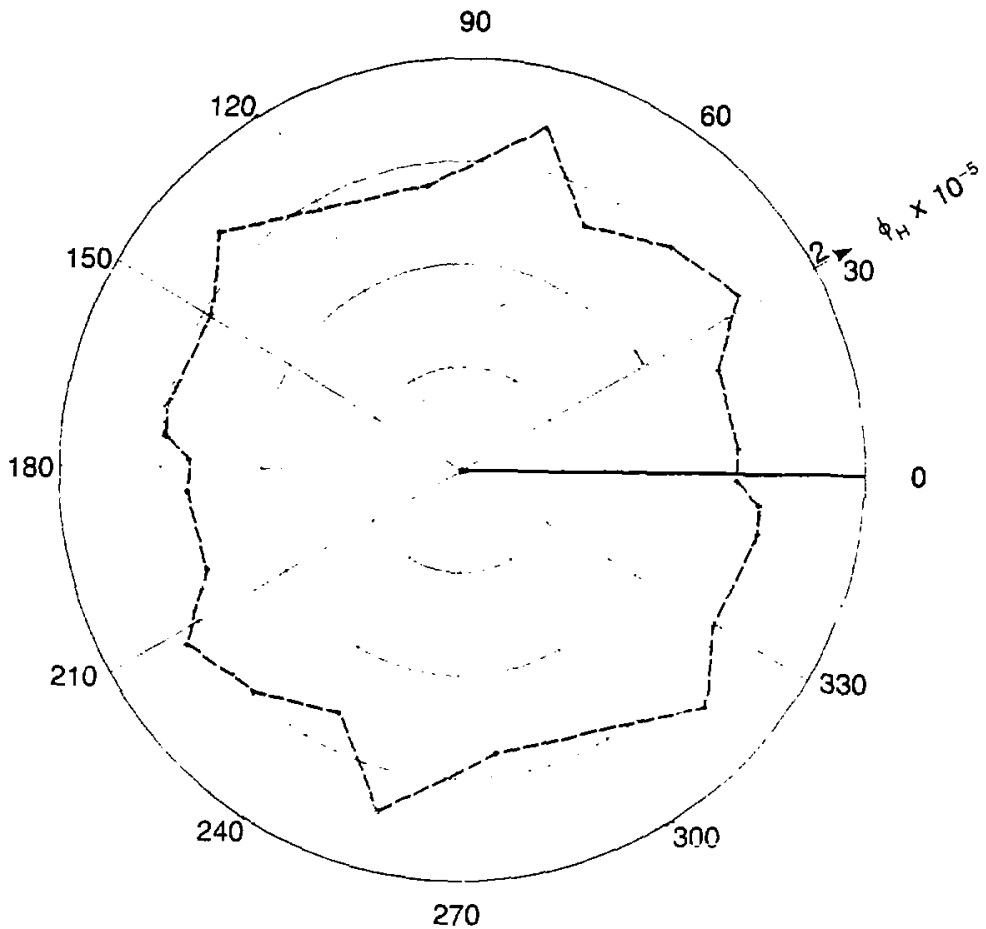

XBL $841-384$

Figure 7-11 Polar Plot of Hydraulic Effective Porosity for Syster. With Mean Fracture Length of $50 \mathrm{~m}$ Using Square Flow Region of Width $33 \mathrm{~J} \mathrm{~m}$. 


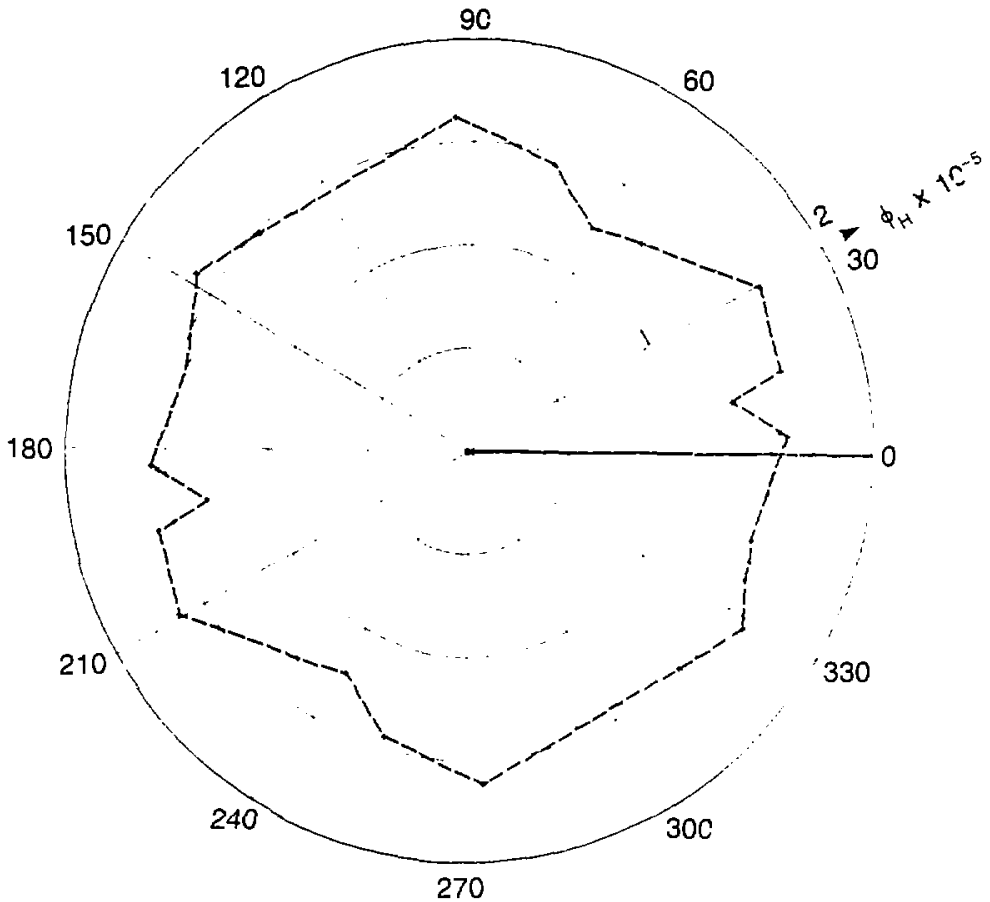

KBL $841-383$

Figure 7-12 Polar Plot of Hydraulic Effective Porosity for System :Vith Mean Fracture Length of $50 \mathrm{~m}$ Using Square Flow Region of Width $175 \mathrm{~m}$. 
width $60 \mathrm{~m}$. The mean hydraulic effective porosity of 03000118 is less than computed at the two larger scales. The $\phi_{\mathrm{H}}$ curve deviates from a circle as large fluctuations in $\phi_{\mathrm{H}}$ are observed near $110^{\circ}$. The standard deviation in $\phi_{H}$ has increaseci to $1.73 \times 10^{-6}$. Thus, a flow region of this size behaves like a discontinuum for transport. As the flow region size decreases, the polar plot of $\phi_{\mathrm{H}}$ begins to deviate from a circle and $\phi_{\mathrm{H}}$ fluctuates with direction. As a consequence, the standard deviation in $\phi_{\mathrm{H}}$ increases.

\subsection{DISTRIBUTED APERTURE LENGTH-DENSITY SERIES}

\subsubsection{Distributed Aperture Length-Density Study with Standard \\ Deviation Equal to Mean Aperture}

The previous constant aperture length-iensity study investigated mechanical transport caused by the configuration of the fracture network, ignoring heterogeneity that results by distributing apertures. In the first disiributed aperture length-density study, fracture apertures were distributed using two techniques.

1) Apertures were lognormaily distributed with a mean of $0.00005 \mathrm{~m}$ and a standard deviation of $0.00005 \mathrm{~m}$.

2) Apertures were linearly correlated to fracture length in the following vay:

$$
\mathrm{b}=\frac{\mu_{1}}{\mu_{\mathrm{b}}} \mathrm{l}+\epsilon
$$

where $x$ is a random variation in aperture and $\mu_{\mathrm{b}}$ is equal

to $0.00005 \mathrm{~m}$.

Since fracture lengths were lognormally distributed with $\mu_{\text {l }}$ equal to $\sigma_{1}$, the linear correlation model was used so that $\mu_{b}$ would equal $\sigma_{b}$ similar to the lognormal distribution of apertures.

\subsubsection{Continuous Fracture System}

The fracture apertures were lognormally cistributed for the continuous fracture system since fracture lengths which are infinitely long cannot be correlated with apertures. A Monte Carlo study was conducted because fracture apertures were probabilistically simulated. Thi 


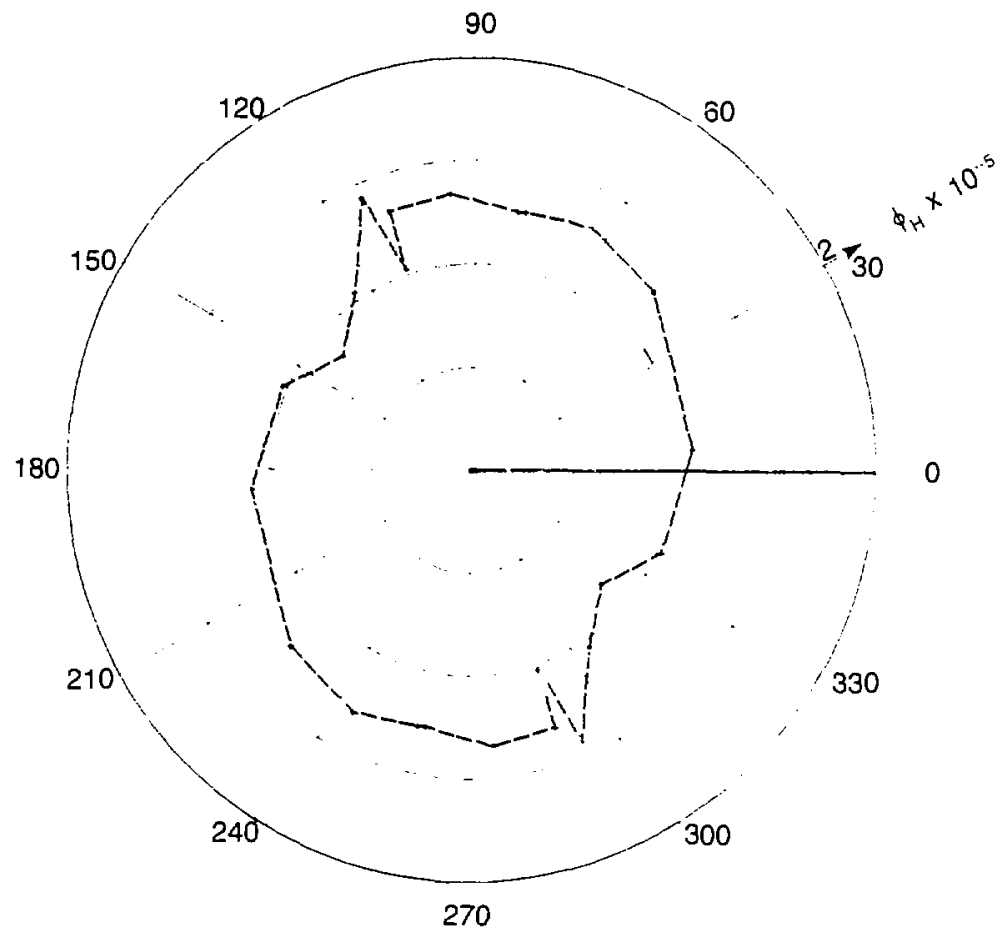

$\times B L 34 !-385$ 
continuous fracture system in each realization was created in the same way as in the constant aperture length-density study except that apertures were lognormally distributed. Figure 7-14 shows the fracture pattem in the generation regios for one of the realizations. The intensity of each fracture (line) is directly related to the fracture's aperture.

The number of realizations for this Monte Carlo study was deternined from: 1) the stability of the mean directional $\phi_{\mathrm{H}}$, and 2) the directional stability of $\dot{\phi}_{\mathrm{H}}$. The mean directional $\phi_{\mathrm{H}}$ was computed in the following way. For each realization, the hydraulic effective porosity in each direction was added up to obtain the sum of all hydraulic effective porosities for this realization. This total was next added to the previously calculated total of all hydraulic effective porosities. Mean directional hydraulic effective porosity was then compuizd by dividing the last total by the total number of measurements of hydraulic effective porosity. We expected that $\phi_{\mathbf{H}}$ would be directionally stable as was found earlier in the constant aperture lengthdensity study. When $\phi_{H}$ is directionally stable, the mean directional $\phi_{H}$ is equal to its directionally stable value.

Hydraulic effective porosity in each direction should converge to its stable ;alue as the number of realizations increases. The directional stability of $\phi_{\mathrm{H}}$ was tested using the polar plot of mean $\phi_{\mathrm{H}}$ - When $\phi_{\mathrm{H}}$ is directionally stable, the polar plots of $\phi_{\mathrm{H}}$ for $n$ realizations and $n+5$ realizations are identical. The mean directional $\phi_{H}$ tests the overall stability of the hydraulic effective parosity. The directional stability of $\phi_{\mathbf{H}}$ requires that in every direction hydraulic effective porosity is stable.

Figure $7-15$ is the ploi of the mean directional hydraulic effective pornsity versus the number of rcalizations. Initially, this mean $\phi_{\mathbf{H}}$ increases rapidly as the number of realizations increase. Mean directional hydraulic effestive porosity is then relativaly constant for the neat eight realiza' „uns. At realization 12, ancther sudden increase in this mean $\phi_{H}$ is measured. This sudden increase is followed by a gradual decrease in mean directional $\phi_{\mathrm{H}}$ as the number of realizations increases. Mean directional hydraulic effective porosıty is slowly approaching a stable value. This stable value is not equal to the total porosity of the system which is 


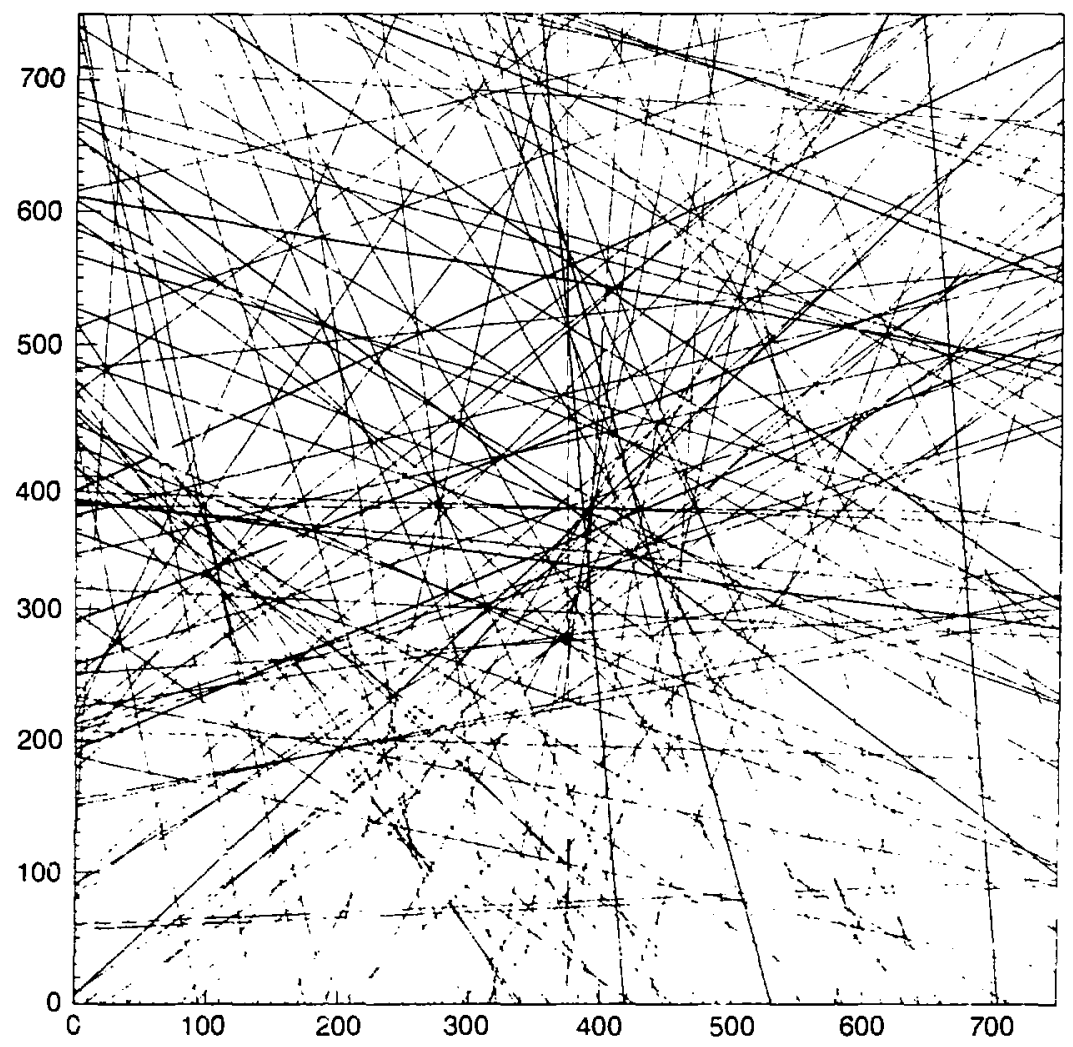

(m)

$\times B L 8311-3457$

Figure 7-14 Fracture Network in the Generation Region for Continuous System in the First Distributed Aperture Study. 


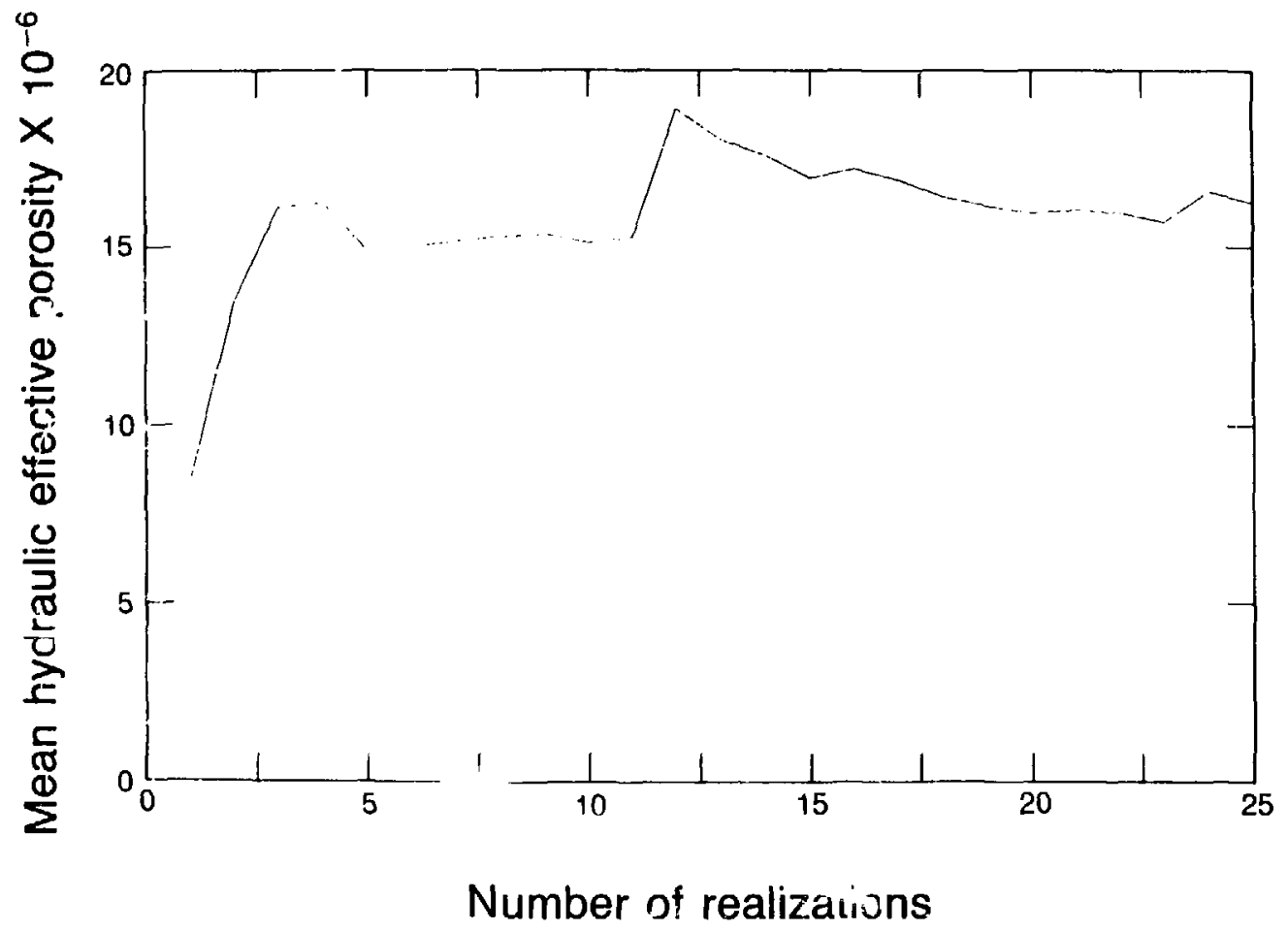

Figure 7-15 Mean Hydraulic Eflective Porosity in All Directions Versus the Number of X8L 841-534 Realizations. 
0.0000106 .

The standard error of the mean directional $\phi_{\mathrm{H}}$ is plotted against the number of realizations in Figure 7-16. The large increases in the standard error of the mean directional $\phi_{H}$ at realizations 2 and 12 reflect the two sudden increases in this mean $\phi_{\mathrm{H}}$. Ifter realization 12 , the standard error slowly decreases as the number of realizations increases. This figure shows that in one out of ten realizations a fracture system is created in which the hydraulic effective is very large. Cozsequently, both mean directional $\phi_{\mathrm{H}}$ and its standard eiror increase a: this realization. This sudden increase is followed by a decrease and a slow stabilization in mean directional $\phi_{H}$.

The sudden increase in mean directional $\phi_{\mathrm{H}}$ at realization 12 is caused by two fracturcs with very large apertures (super conductors) within the fracture network (Figure 7-17). These two large apertures wele created because of the large standard deviation in fracturt apertures. Since travel time in a fracture is proportional to $b^{-2}$, fluid flowing in the large aperture tractures had a much smaller travel time from side 2 to side 4 as compared with the travel times for the rest of the fluid. Thus, two zones of contrasting fluid movement developed in the flow region. This type of transport is called inhomogeneous transport. The breakthrough curve for direction of flow $20^{\circ}$ (Figure 7-18) shows that part of the fluid moves within a zone of fast movement, and have travel times that are less than $\bar{t}$. The remaining fluid moves within a zone of slow movement and have tra : times that can be $\mathrm{mb}^{\mathrm{h}} \mathrm{h}$ larger than $\overline{\mathrm{t}}$. The two largeaperture fractures also caused a large increase in specific discharge because $q$ is proportional to

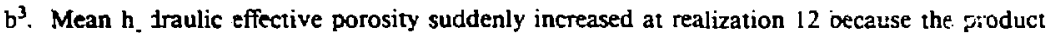
of $q$ and $\bar{\tau}$ was large in relation to $L$

Figures $7-19$ and $7-20$ show polar plots of mean $\phi_{H}$ after $5,10.15$, and 25 realizations. After 5 and 10 realizations (Figure 7-19), $\phi_{\mathrm{H}}$ exhibits large directional variations . i.tch polar plot is very jagged. The :wo polar plots in Figure $7-20$ ior 15 and 25 realizations are more simiiar to each other than the two polar plots in Figure $7-i 9$ for 5 and 10 realizations. This indicates that $\phi_{\mathbf{H}}$ is slowly converging to its stab!e value in all directions as the nurnber of reali- 


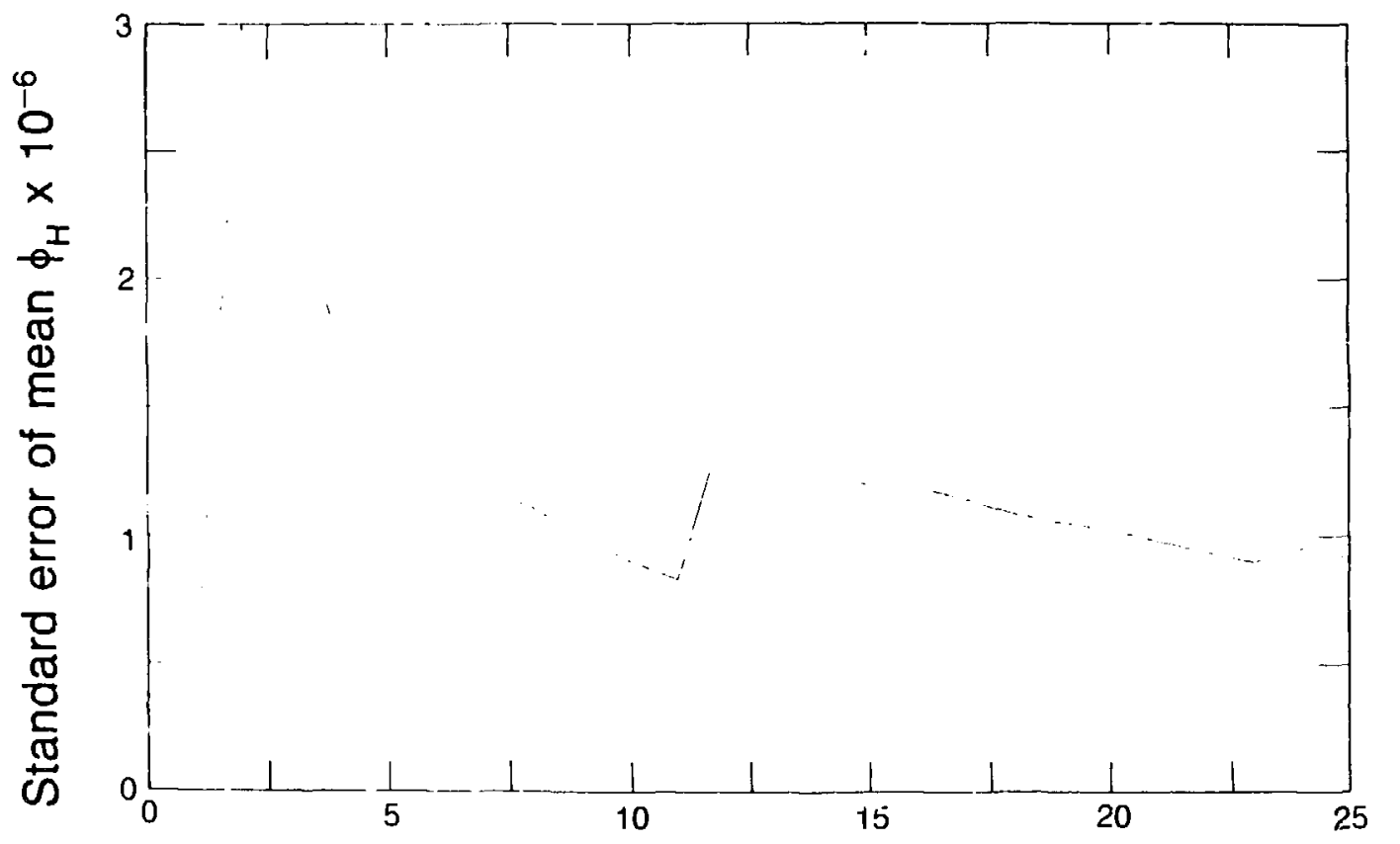

\section{Number of realizations}

Figure 7-16 Standard Error in Mean Hydraulic Effective Porosity Versus the Number of XBL 8311.7386 Realizations. 


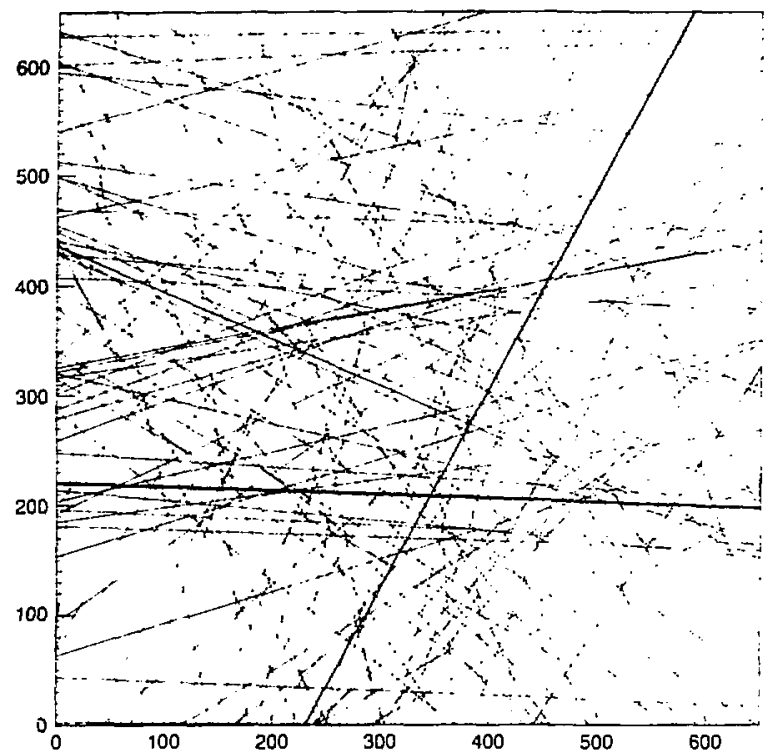

(m)

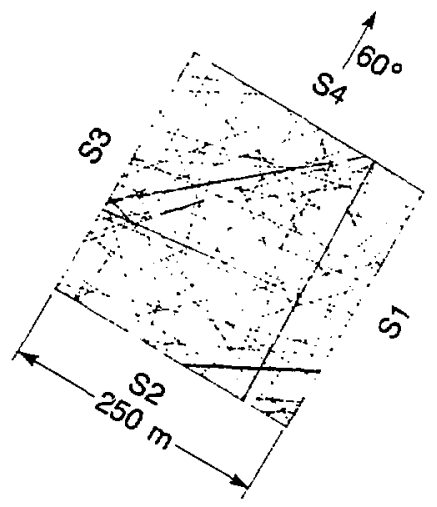

X日L 8011.3458

Figure 7-17 Fracture Network in a) Generation Region and b) Flow Region Oriented at $60^{\circ}$ for Realization 12 of the Continuous System in the First Distributed Aperture Study. 


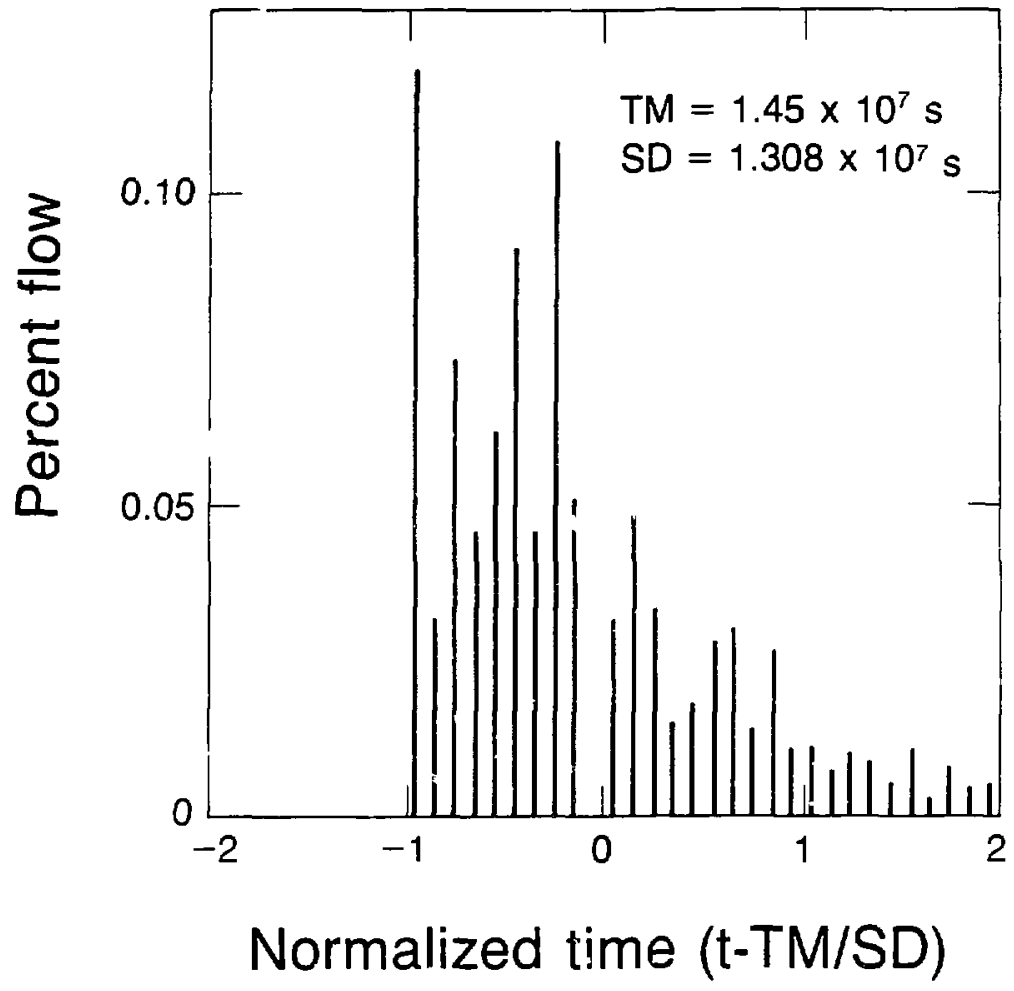

$X B L 8311-3455$

Figure 7-18 Breakthrough Curve for Direction of Flow $120^{\circ}$ for Continuous System in the First Distributed Aperture Study. 

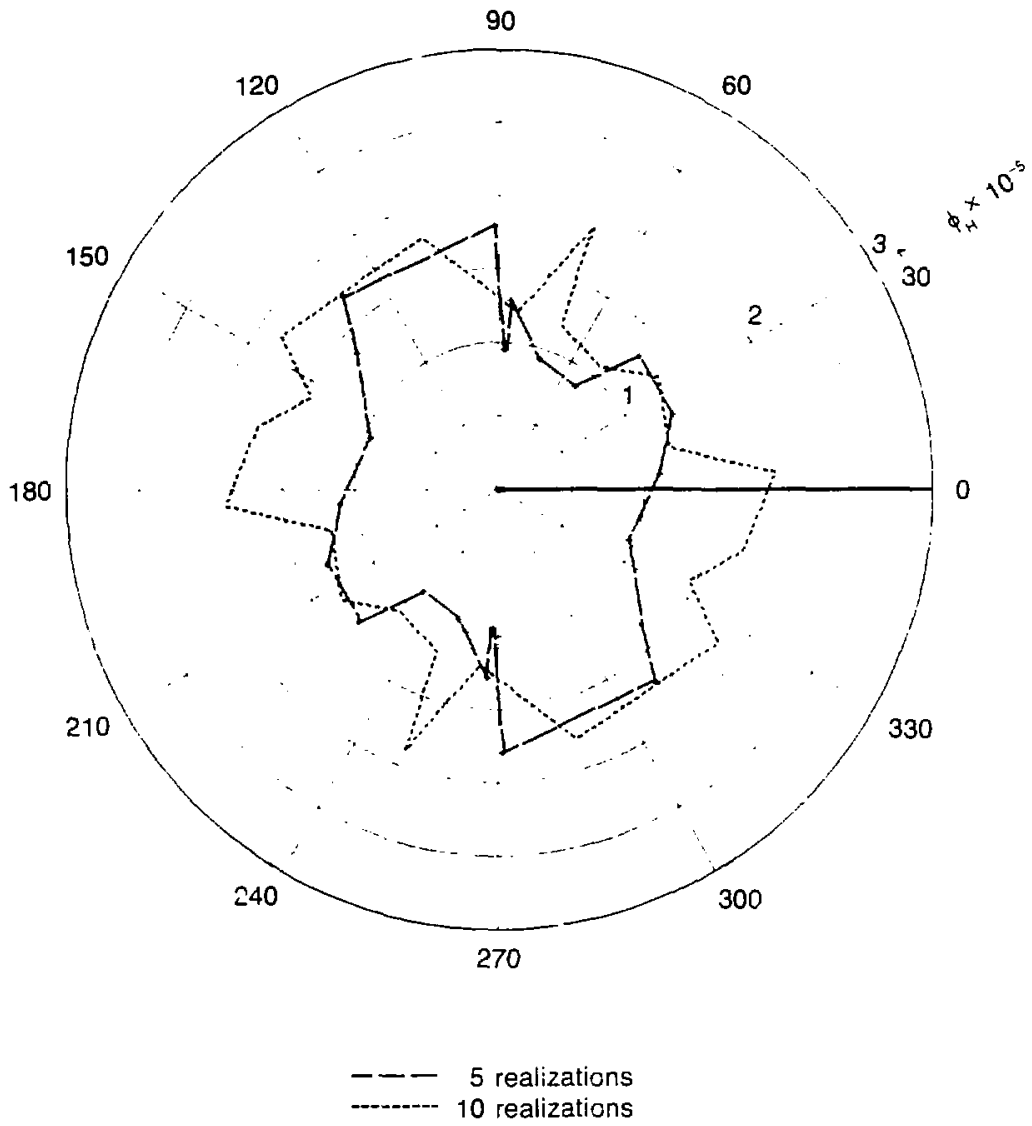

$X B\llcorner 8312 \cdot 7420$

Figure 7-19 Polar Plots of Mean Hydraulic Effective Porosity After Five Realizations and Ten Realizations. 
$-163$.

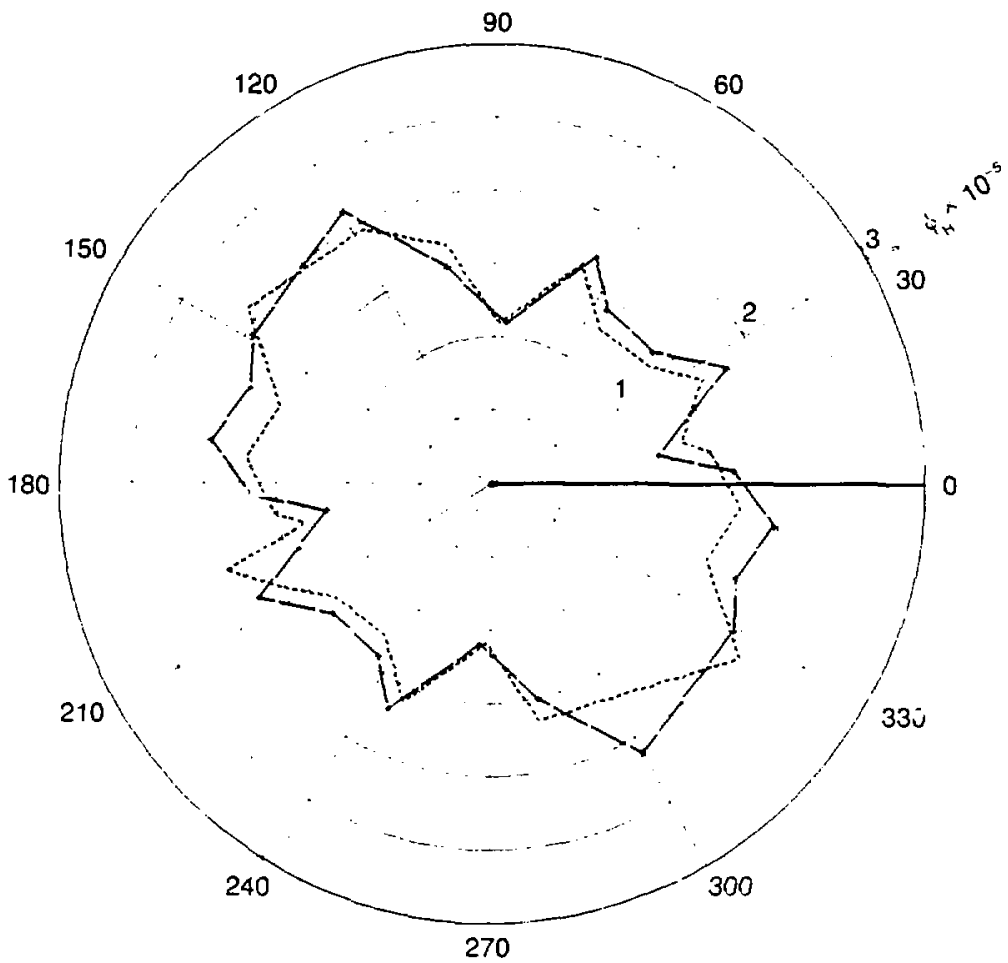

$\begin{array}{ll}- & 15 \text { realizations } \\ --- & 25 \text { realizations }\end{array}$

XBL 83127423

Figure $7-20$ 
zations increase. The Monte Carlo simulation ended after 25 realizations because both mean directional hydraulic effective porosity and directional $\phi_{H}$ were stabilizing. However, after 25 realizations, hydraulic effective porosity is still directionally dependent, and the polar plot of $\phi_{\mathbf{H}}$ is very jasged.

The polar plot of $\phi_{\mathrm{H}}$ after 25 realizations indicates that at this scale the fracture system does not behave like an equivalent porous medium for transport. The characteristics of this polar plot are similar to the polar plot of $\phi_{\mathbf{H}}$ for the discontinuous system of constant aperture with $\mu_{1}$ of $50 \mathrm{~m}$ calculated using square flow regions of width $60 \mathrm{~m}$. At a scale of $60 \mathrm{~m}$, the discontinuous system did not behave like a continuum for transport. The polar plot of $\phi_{\mathrm{H}}$ showed large fluctuations, and deviated from iie nearly circular plot. $'{ }^{c} \phi_{H}$ found using larger flow regions of widths $175 \mathrm{~m}$ and $330 \mathrm{~m}$. For this cortinuous fracture s'stem, the flow region size was too small to be a good statistical sample for the distribution of aperures. Consequently, equivalent porous medium behavior for transport was not obtained at the scale of 250 m. Unfortunately, we could not increase flow region size becaise of the limitations of computer storage.

The polar plots of $\phi_{\mathrm{H}}$ were constructed using flow regions of wid $t h 250 \mathrm{~m}$. These polar plots show that $\phi_{\mathrm{H}}$ is directionally dependent and that $\phi_{\mathrm{H}}$ is greater than $\phi$ in all directions. Walter et al. (1983) reported large differences in travel times, depending on direction of flow, for tracer tests conducted in a fractured aquifer. Waiter et al. questioned the meaning of "effective" porosity. We have shown that $\phi_{\mathrm{H}}$ can be directionally dependent when a frac ure system does not behave like an equivalent porous medium. In porous media, the mean rate of advection is often predicted by $q / \phi$. This estimate would be two times faster than the mean rate of a Thus, the porous medium esimate $q / \phi$ is not a good estimate of the mean rate of advection in all directions at this scale. When field tracer tests are conducted at this scale, one should bear in mind that the transport properties may be directional.

The maximum $\phi_{H}$ occurs near direction of flow $120^{\circ}$. This direction coincides with the 
direction of maximum at for the discontinuous system of constant aperture with $\mu_{1}$ of $50 \mathrm{~m}$. Lo.ngitudinal geometric dispersivity is large when the stanciara deviation of the breakthrough curve is large in relation to $\overline{\mathrm{t}} \mathrm{A}$ large standard deviation can be caused by inhomogeneous transport. The contrasting zones of movement lead to a wide distribution of travel times, and consequently, a large standard deviation for the breakthrough curve. For the discontinuous system of constant aperture with $\mu_{j}$ of $50 \mathrm{~m}, \phi_{H}$ was slightly larger than $\phi_{\mathrm{R}}$, so a small degree of inhomogeneous transport occurred. The movement of fluid in the contrasting zones caused by inhomogeneous transport did not affec: the directional nature of $\phi_{\mathrm{H}}$, as hydraulic effective porosity was directionally stable. However, inhomogeneous transport had a major influence on $\sigma^{2}$, as the spread in the breakthrough curve was greatest in direction of flow $120^{\circ}$. A larger deviation between $\phi_{\mathrm{H}}$ and $\hat{\psi}_{\mathrm{R}}$ was measured for this continuous fracture system in this direction. Thus, a greater degree of inhomogeneou: ansport occurred because of the heterogeneity created by distributing fracturt apertures. Unlike the discontinuous fracture system, inhoñogeneous transport had a major directional effect on $\phi_{\mathrm{H}}$ for this continuous fracture system.

Equivalent porous medium flow behavior can oe analyzed using DEVF and DEVA. Figure $7-21$ is a plot of mean DEVA and mean DEVF varsus direction of flow after 25 realizations. DEVF is much larger for this continuous system than for the discontinuous system of two sets oriented at $0^{\circ}$ and $60^{\circ}$. A larger difference in flow rate on opposing sides of the flow region occurred because of the wide distribution in fracture apertuses. A large-aperture fracture which intersects side 2, but does not intersect side 4 can cause this large flow difference. Thus, it is likely that this continuous fracture system does not behave like an equivalent pcrous medium for fluid fow at this scale. It will be recalled that the directional flow characteristics for the discontinuc is system behaved like an equivalent porous medium.

Figure $7-22$ is a polar plot of mean tortuosity after 25 realizations. The tortuosity curve is nearly circular which indicates that the medium is isotropic. Tortuosity is the only mechanical transport parameter that is similar in both the constant aperture and the distributed aperture length-density series. This result is not surprising. since the sensitivity analysis in Chapter 6 


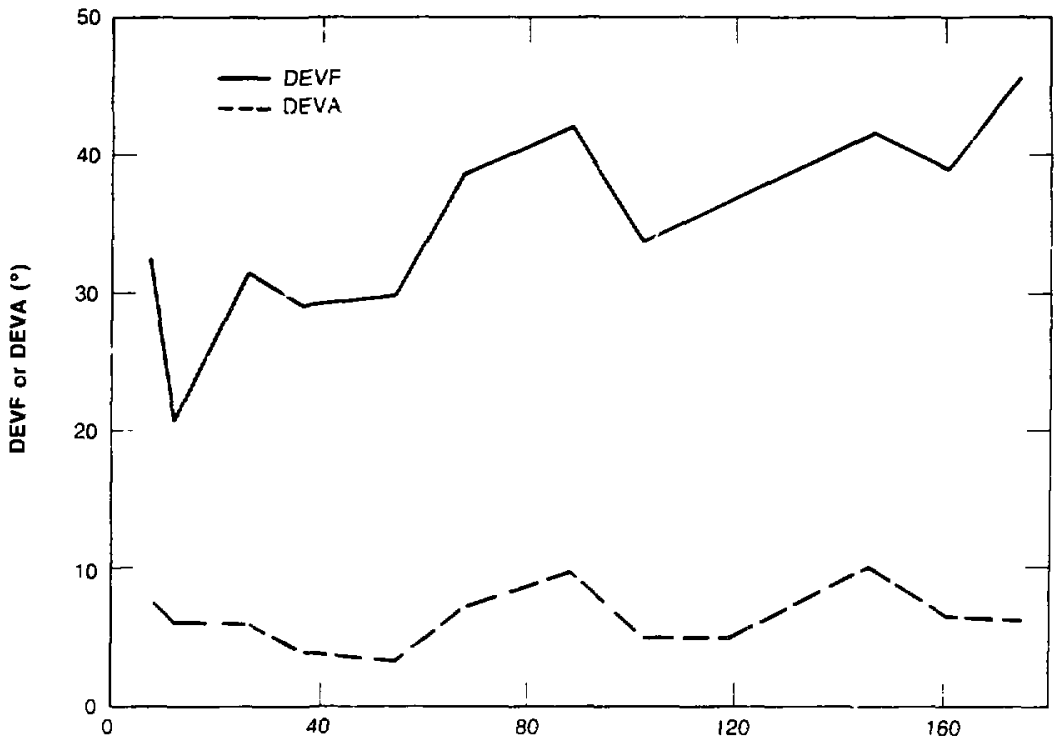

Direction of flow $\left({ }^{\circ}\right)$

XBL $B 401-6788$

Figure 7-21 Mean DEVF and Mean DEVA Versus Direction of Flow for Cont.unous Syster: in the First Distributed Aperture Study. 


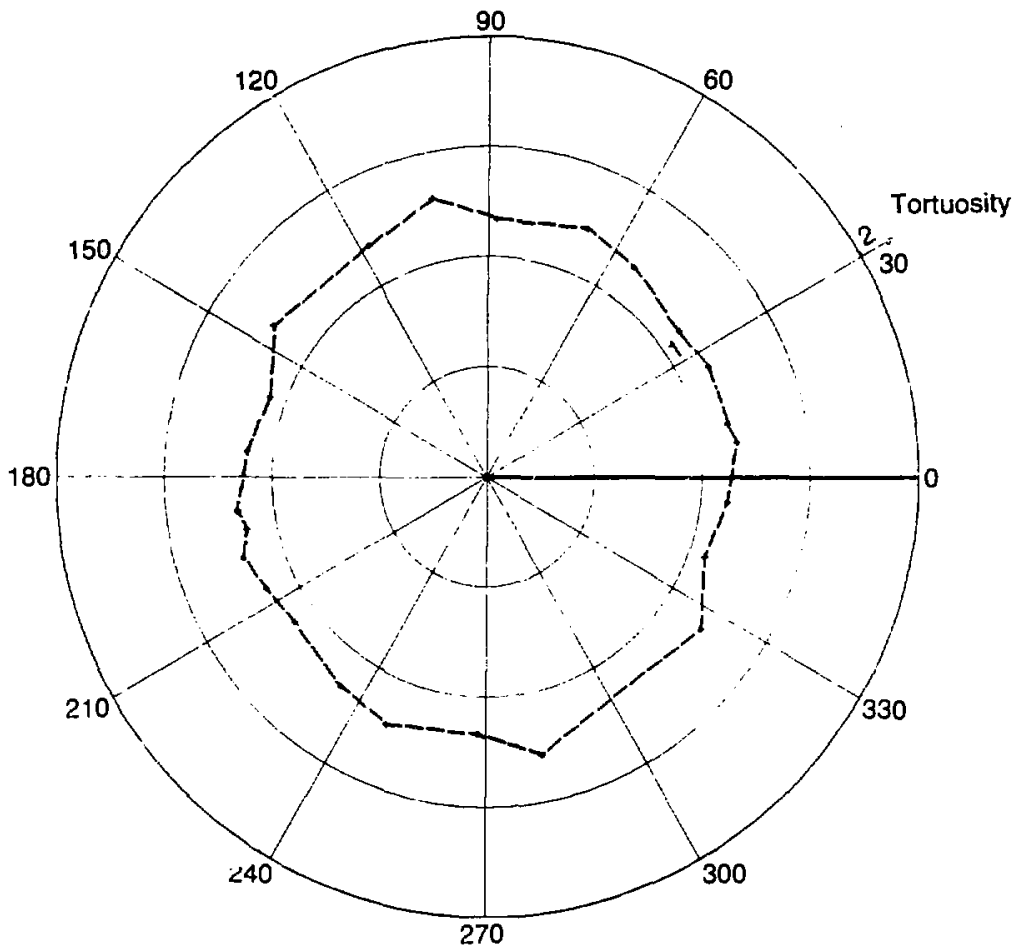

XaL 84016793

Figure 7-22 Polar Plot of Mean Tortuosity for Continuous System in the First Distributed Aperture Study. 
showed that 1ortuosity was insensitive to both $\mu_{\mathrm{b}}$ and $\sigma_{\mathrm{b}}$.

In most realizations, longitudinal geomeuic dispersivity could not be computed in any direction or fiow because $\sigma^{2} / t^{2}$ exceeded unity. The variance in the breakthrough curve was very large because of the wide distribution ir. fracture apertures $\left(\mu_{\mathrm{b}}=\sigma_{\mathrm{b}}\right)$. Travel time in a fracture is proportional to $\mathrm{b}^{-2}$. Thus, the distribution in travel times was much wider than the distribution of apertures. Consequantly, the ratio of $\sigma$ to $\bar{t}$ exceeded unity, which meant that $\mathrm{M}_{\mathrm{L}}$ approached infinity. No poiar plot of $\alpha_{L}$ was constructed because of the limited data.

\subsubsection{Discontinuous System witi Mean Fracture Length of $50 \mathrm{~m}$.}

\subsubsection{System with Lines.rly Correlated Apertures}

The fracture system for each realization was creaied the same way as in the constantaperture study except that apertures were linearly correlated with fracture lengths by equation 7.2. The linear correlation model was used to distribute apertures sn that: 1) long fractures would be assigned large apertures and short fractures would be assigned small apertures and, 2) the mean aperture of $0.00005 \mathrm{~m}$ would equal the standard deviation of aperture. This mean was equal to the standard deviation because fracture lengths were lognormaily distributed with the mean of $50 \mathrm{~m}$ equal to the standard deviation of fracture length. Square flow regions of width $330 \mathrm{~m}$ were oriented at svery $15^{\circ}$ so that mechanizal transport could be studied.

Numerical precision problems were encountered in the mechanical transport stage of the computer program because of large hydraulic gradient differences in the elements intersecting a node. For example, consider two elements intersecting a node. Suppose element 1 has an aperture which is 30 times larger than the aperture of element 2. For this fracture system the large aperture difference is caused by the wide distribution of apertures. Since both fractures have the same flow rate, the ratio of hydraulic gradients in the two elements is proportional to $b^{3}$ :

$$
\frac{(\nabla \Phi)_{2}}{(\nabla \Phi)_{i}}=(30)^{2}=27000
$$

Corsequently, it was difficult for the program $t \cdot 3$ distinguish no now elements (sero gradient) from low flow elements (very small gradient). This problem is similar to the proble $n$ one 
encounters when modeling transient flow in an aquifer consisting partly of sand and pariy of clay. Sand is much more permeable than clay. So, small time steps must be used in order for the numerical soiution to be stable. Also, the conductive matrix for such a problem is a stiff matrix because the coefficients along the diagonal of this matrix vary significantly. Numerical solution is often difficult when the conductance matrix is a stiff matrix.

The problem of large hydraulic gradient differences at a nocic was not encountered in the study of the continuous fracture system of distributed apertures. Theoretically, the gradient along an infinitely-long fracture is equal to the product of the magnitude of the hydraulic gradient and the cosine of the angle between the fracture orientation and direction of the gradient. Since the gradient along the fracture was not related to aperture cubed, there were no extreme difèrences in hydraulic gradient: at a node. For this system, with $\mu_{1}$ of $50 \mathrm{~m}$ and linearly correlated apertures, five realizations were run for each oriertation.

The rock effective porosity, hydraulic effective porosity, and total porosity are each shown in Figure 7-23. Both th.al porosity and rock effective porosity were directionally stable, but hydraulic effective porosity was highly directionally dependent. Hydraulic efizctive porosity did not show the characteristics of an equivalent porous medium, as the polar plot of $\phi_{H}$ was very jagged and $\phi_{\mathrm{H}}$ showed large directional variations. These porosity results were based on five realizations, and consequently, may not be conclusive. However, we believe that hydraulic effective porosity will still exhibit directional tendencies when a large number of realizations are made because the same directional tendencies for $\phi_{\mathrm{H}}$ were present after 5 and 25 realizations for the previous continuous system. The direction of maximum $\phi_{\mathrm{H}}$ was near $120^{\circ}$. This direction was also the direction of maximum $\phi_{\mathrm{H}}$ for the continuous fracture system with distributed apertures, and the cirection of maximum $\alpha_{i}$ for the discontinuous system with $\mu_{l}$ of $50 \mathrm{~m}$ and constant apertures. The maximum $\phi_{H}$ had a value four times larger than the rock effective porosity. Consequently, the deviation between $\phi_{H}$ and $\omega_{Z}$ is greater for this system 'han for the previous cintinuous system.

riydraulic effective porosity was greater tnan total porosity because both $q$ and $T$ were 


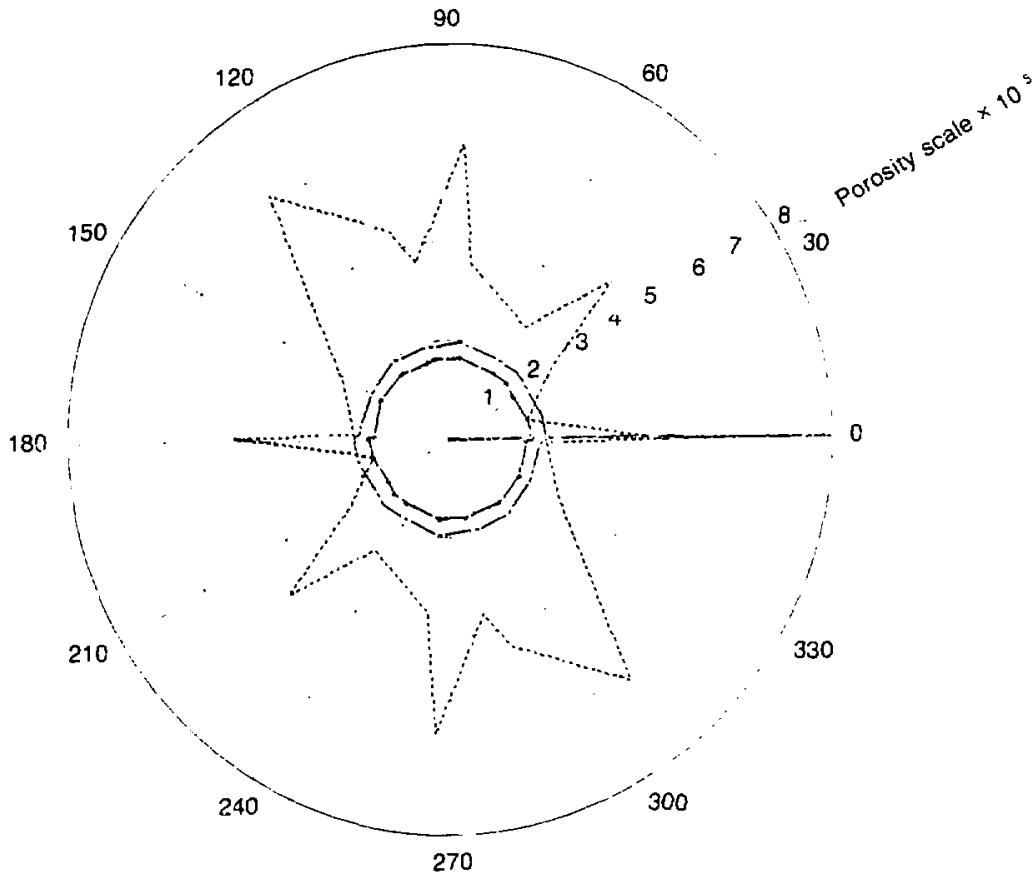

_- - Rock effective porosity
-...-. Hydraulic eifective porosity
-._- Total porosity

XBL 83127419

Figure 7-23 Polar Plots of Total Porosity, Hydraulic F Tective Por sity, and Rock Effective Poiosity for Discontinuous System Wirh Mean Fracture Length of $50 \mathrm{~m}$ and Linearly Correlated Apertures 
large in relation to $\mathrm{L}$. Specific discharge was large because long fractures with large apertures have a greater probability of conducting flow (intersecting other long fractures) than the short fractures with small apertures. Mean travel time was large because of the slow movement of part of the fluid. Figure $7-24$ shows a breakthrough curve in a direction in which $\phi_{\mathbf{H}}$ was three times larger than $\phi_{\mathrm{R}}$. Sixty-eight percent of the fluid arrived within the small time saterval between the normalized times of -0.5 and 0.0 . Between the normalized times of 0.0 and 0.5 , another 22 percent of the fluid arrived. However, the 10 percent of the fluid that arrived between the normalized times of 0.5 and 2.0 caused the mean travel time to be large. The real time $(t)$ is equal to:

$$
\mathbf{t}=\mathbf{t}+\sigma(\text { normalized time })
$$

Since the standard deviation of this breakthrough curve is larger than the mean travel time, real time rapidly becomes greater than $\vec{t}$ as normaiized time increases. The right skewness in the breakthrough curve reflects the movement of paricles which had large travel times.

Mean DEVA and mean . DEVF are shown plotted against direction if sow in Figure 7-25. Both DEVA and DEVF were much larger in this system than in any of the previous systems studied. The large DEVA and DEVF were caused by the heterogeneity of aperiures. ror the iracture system in the fifth realization of the constant aperture study with $\mu_{l}$ of $50 \mathrm{~m}$, mean directional DEVA and DEVF were only $10.5^{\circ}$ and 12.4 , respectively. The large values in DEVA and DEVF at this scale show that this fracture system does not behave iike an equivalent porous med.um for hluid flow.

Figure 7-26 is a pols plot of mea: tortuosity. Tortuosity is nearly constant in all cirections, and the mean directional tortuosity is 1.28 . Thus, even though hydrauiic effective porosity showed large directional variations, tortuosity showed the same directional behavior as found in the constant-aperture study for the fracture system with $\mu_{1}$ of $50 \mathrm{~m}$. where the polar pjot of hydra 'ic effective was nearly circular.

Longitucinal geometric dispersivity could not be iomputed for more than 90 percent of the realizations. The maan velocity within an element varizd considerably in the flow region 


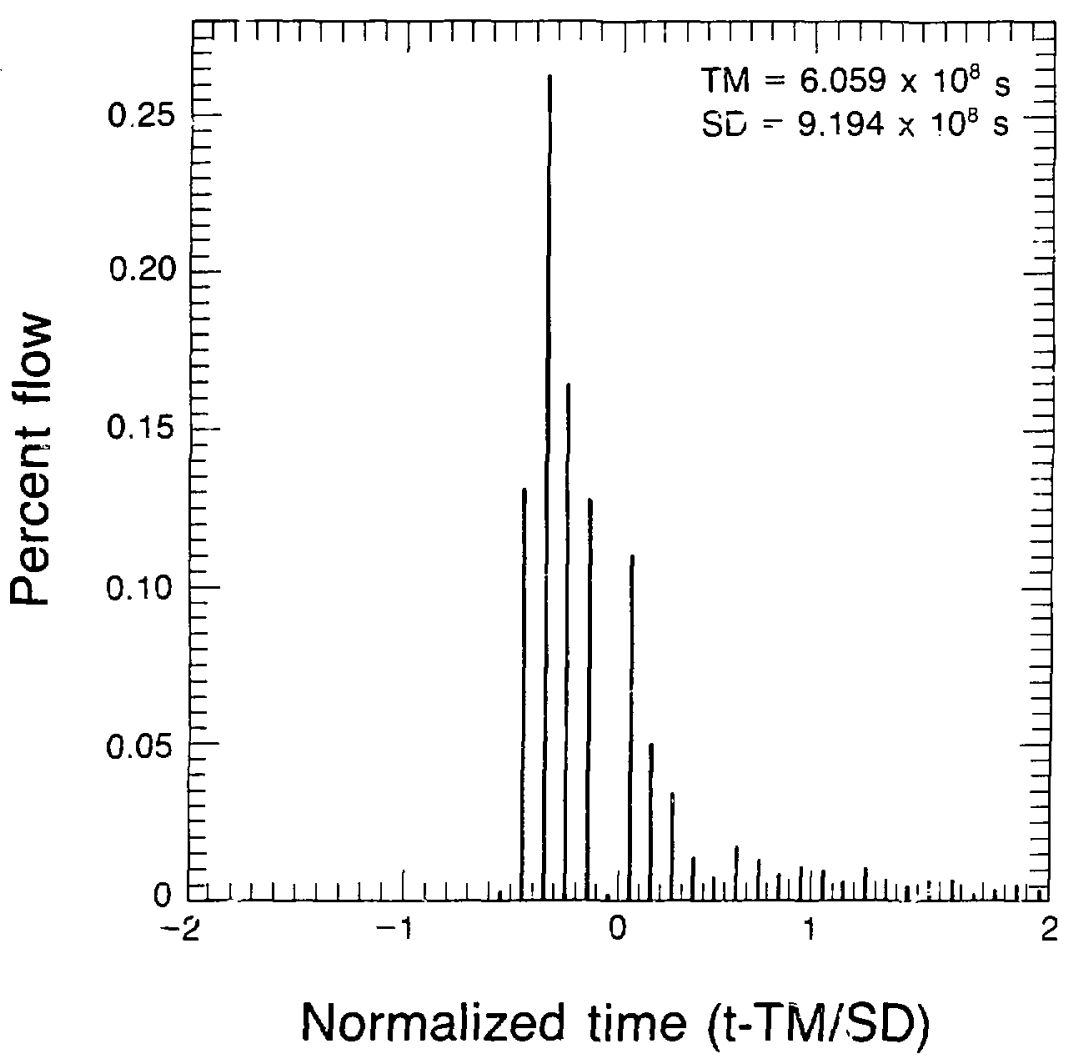

$\times$ ค -83127422

Figure 7-24 Breakthrough Curve in a Direction in Which Hydraulic Effective Porosity is Three Time: Larger Than Rock Effective Porosity. 


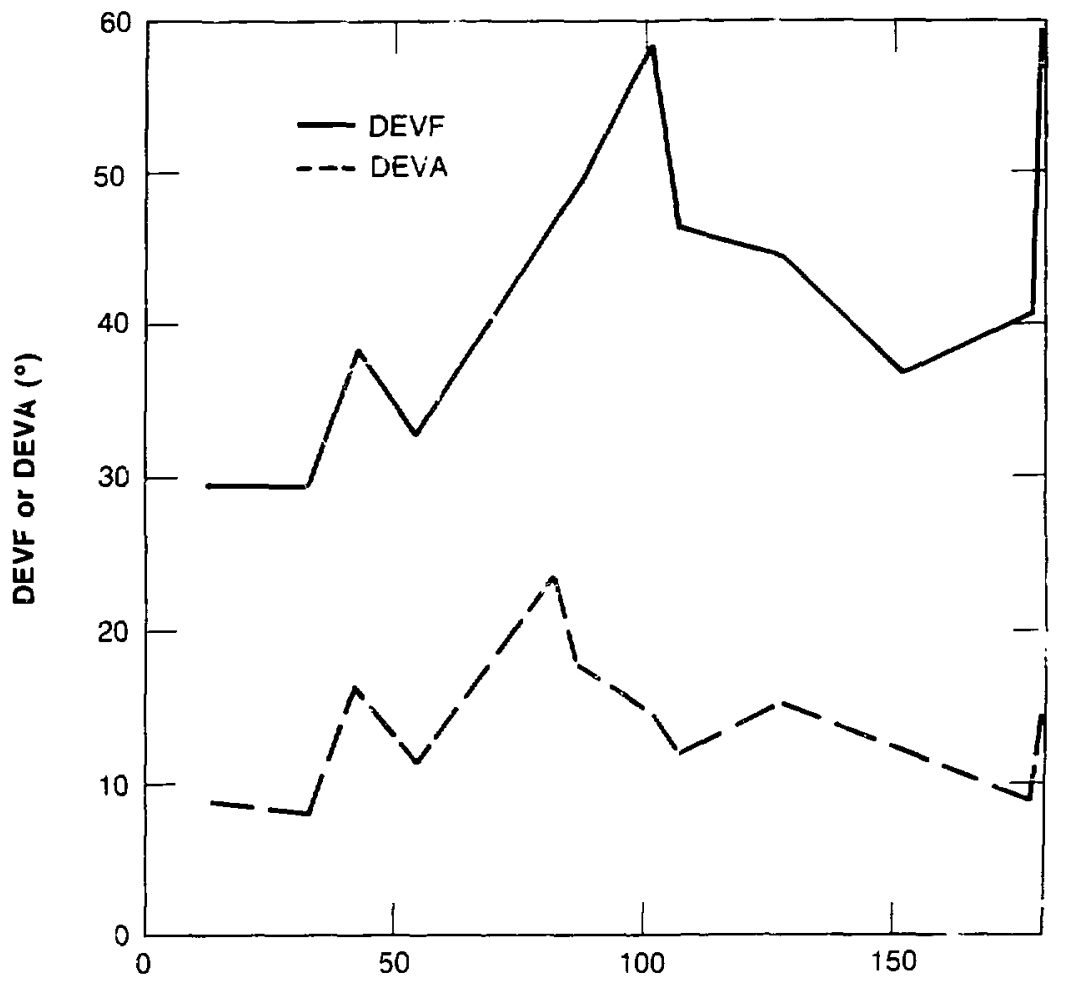

\section{Direction of flow $\left({ }^{\circ}\right)$}

XBL $8401-6787$

Figure 7.25 Mean DEVF a.1d Mean DEVA Versus Direction of Flow for System With Mean Fracture Length of $50 \mathrm{~m}$ and Linearly Correlated Apertures. 


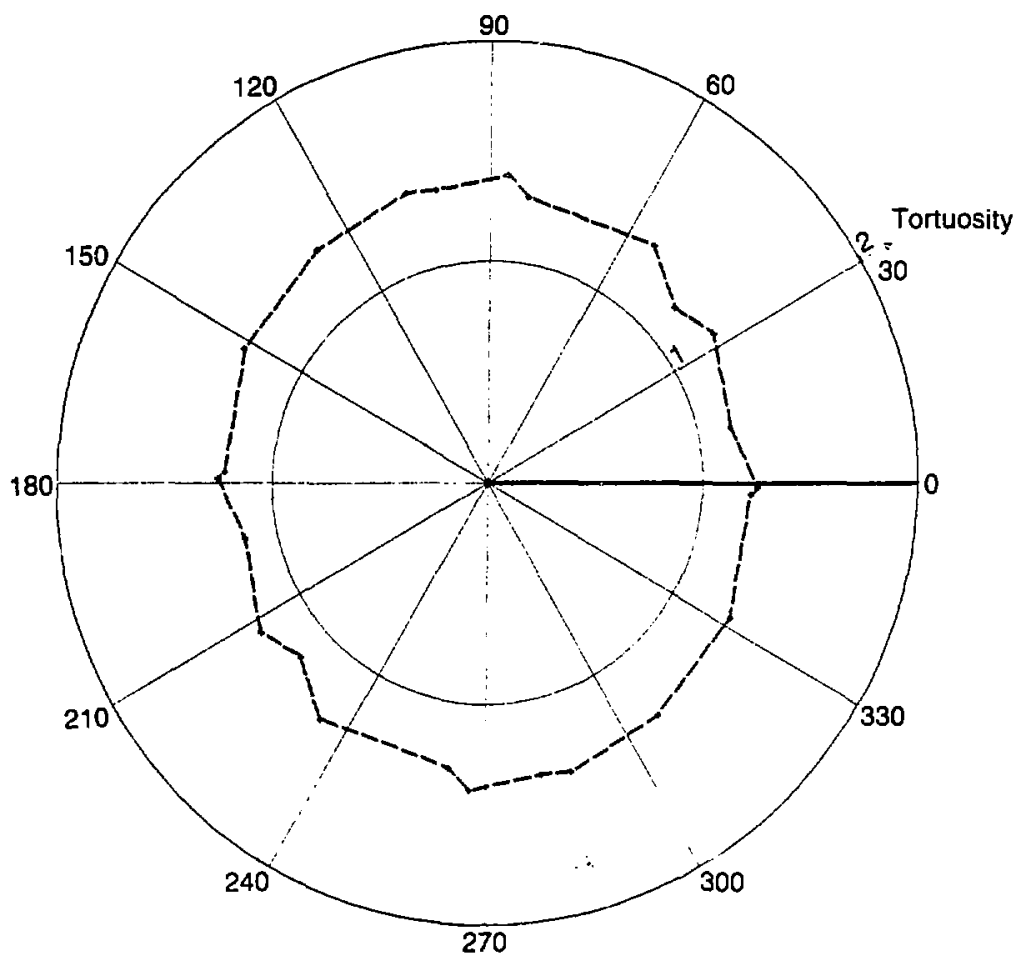

XBL 8401-6794

Figkre 7-26 Polar Plot of Mean Tortuosity for System With Mean Fracture Length of $50 \mathrm{~m}$ and Linearly Correlated Apertures. 
because of the wide range of apertures. This large velocity variation caused fluid particles to spread out rapidly such that $\sigma / \mathrm{t}$ exceeded unity. When $\sigma / \mathrm{t}$ exceeds unity, the Peclet number and $\alpha_{\mathrm{L}}$ approach infinity. Consequently, the rate of dispersion cannot be characterized by $\alpha_{\mathrm{L}}$ using the classical approach. Transport is probably occurring in the initial non-Fickian period where dispersion occurs at a faster rate than in the later Fickian time domain.

\subsection{System with Lognormally Distributed Apertures}

Fractures were created in a generation region of 1000 by $1000 \mathrm{~m}$ by lognormally distributing apertures using a mean of $0.00005 \mathrm{~m}$ and a standard deviation of $0.00005 \mathrm{~m}$. Twelve square flow regions of width $330 \mathrm{~m}$ were oriented at every $15^{\circ}$ within the generation region. For each orientation, two realizations were run to study mechanical transport.

Figure $7-27$ is the polar plot of mean hydraulic eñective porosity. This system showed extreme directional variations in hydraulic effective porosity. Hydraulic etfective porosity was much larger than rock effective porosity. Rock effective porosity and total porosity were both directionally stable at 0.0000072 and 0.0000098 , respectively. The large directional variations in $\phi_{\mathrm{H}}$ indicate that this fracture system cannot be treated like an equivalent porous medium for transport.

Figure 7-28 shows mean DEVA and mean DEVF plotted against the direction of flow. DEVA and DEVF are both very large in most directions. Consequently, this fracture system also does not behave like an equivalent porous medium for fluid flow. Of all the systems studied, this fracture system showed the greatest deviation from porous medium behavior.

\subsubsection{Distributed Aperture Length-Density Study with Standard \\ Deviation of Aperture Equal to 0.3 of Mean Apert ure}

The directional behavior of hydraulic effective porosity was different in the constant aperture and in the first distributed aperture length-density studies. Hydraulic effective porosity was directionally stable in the constant aperture study, such that the fracture systems behaved like equivalent porous media. Large directional variations in hydraulic effective porosity in the 


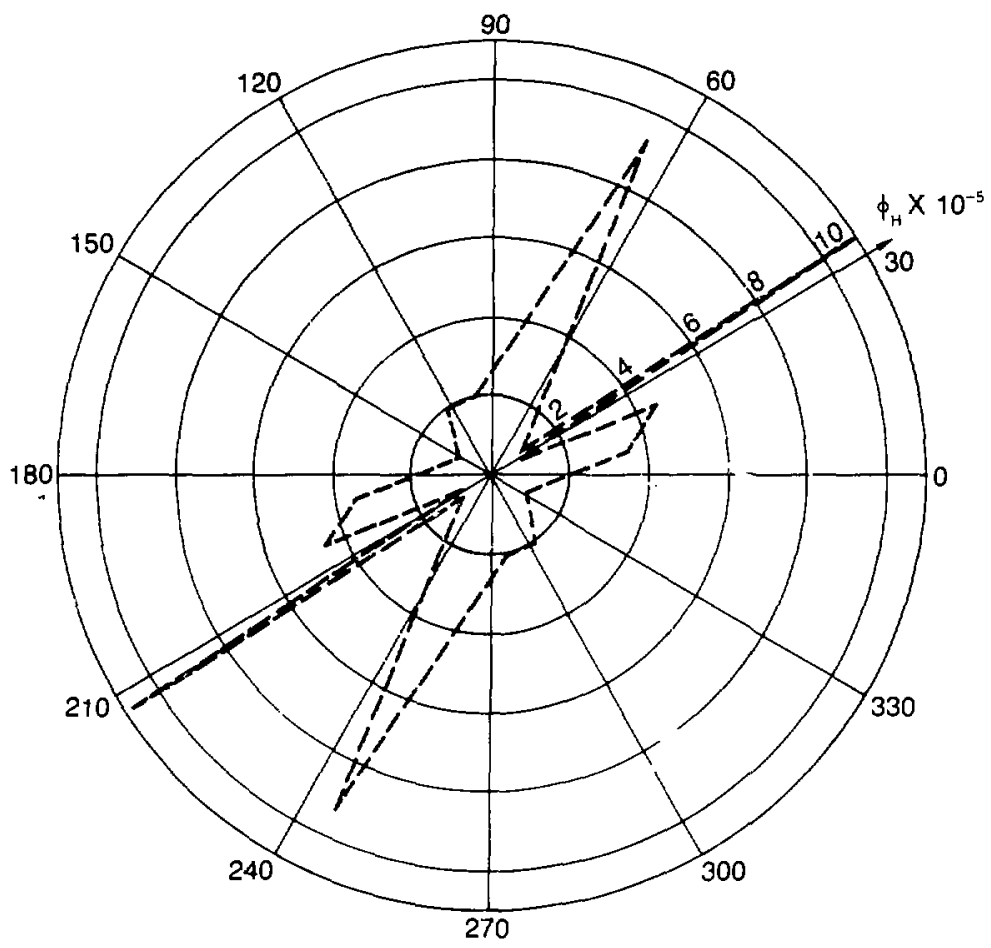

XBL 8401-702

Figure 7-27 Polar Plot of Mean Hydraulic Effective Porosity for System With Mean Fracture Length of $50 \mathrm{~m}$ in the First Distributed Aperture Study. 


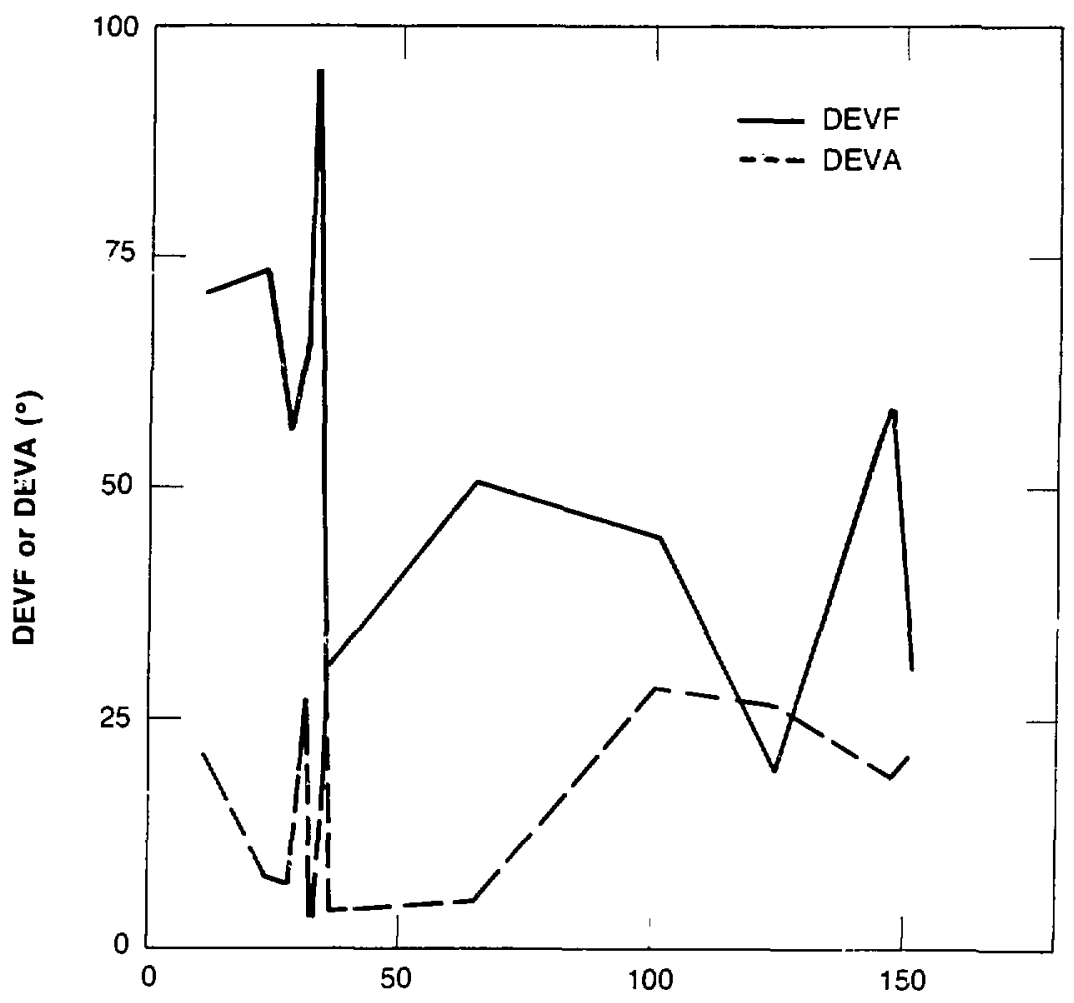

Direction of flow $\left({ }^{\circ}\right)$

XBL 84016790

Figure 7-28 Meen DEVF and Mean DEVA Versus Direction of Flow for System With Mean Fracture Length of $50 \mathrm{~m}$ in the First Distributed Aperture Study. 
first distributed aperture study showed that the fracture systems exhibited no porous media equivalence. A third length-density study was conducted for fracture systems in which the standard deviation of aperture was 0.3 of the mean aperture. Thus, this length-density study bridged together the two previous studies.

The two fracture systems used in this study were the continuous fracture system and the discontinuous system with $\mu_{l}$ of $50 \mathrm{~m}$. Each system was created in the same way as described in the distributed aperture study, except that apertures were lognormally distributed using a mean of $0.0000917 \mathrm{~m}$ and a standard deviation of $0.0000275 \mathrm{~m}$. The mean and standard deviation were determined so that the mean $b^{3}$ was constant in all three length-density studies. For each fracture system, five realizations were nun for each orientation of the flow region to study mechanical transport.

\subsubsection{Continuous Fracture System}

The polar plots of hydraulic effective porosity and total porosity are shown in Figure 7-29. Hydraulic effective porosity is nearly equal to total porosity in all directions. Thus, this fracture system behaves like an equivalent porous medium for transport. We have found that continuous systems with $\sigma_{\mathrm{b}} / \mu_{\mathrm{b}}$ less tha: 0.3 behaved like equivalent porous medium for transport when flow regions of size 250 by $250 \mathrm{~m}$ were used.

Mean DEVA and mean DEVF are plotted against direction of flow in Figure 7-30. DEVF is two times smaller in most directions for this system than for the continuous system with $\sigma_{\mathrm{b}} / \mu_{\mathrm{b}}$ of 1 . The mean directional DEVF and DEVA were 9.35 and $2.12^{\circ}$, respectively, for the continuous system with constant aperture $\left(\sigma_{b} / \mu_{b}=0\right)$. Consequently. porous medium flow behavior is more likely to occur in continuous systems where the ratio of $a_{b}$ to $\mu_{b}$ is small.

Figure 7-31 shows the polar plot of tomuosity. In the three length-density studies, the same directioral behavior in tortuosity was found. The polar plots of tortuosity were nearly circular, indicating that each system was fairly isotropic. Tortuosity decruased slightly as the ratuc of $\sigma_{b} 10$, increased. Tortuosities were $1.261,1.253$. and 1.167 for the continuous systems $u_{3}$ th $\sigma_{b} / \mu_{b}$ oi $0,0.3$. and 1 . respectively. 


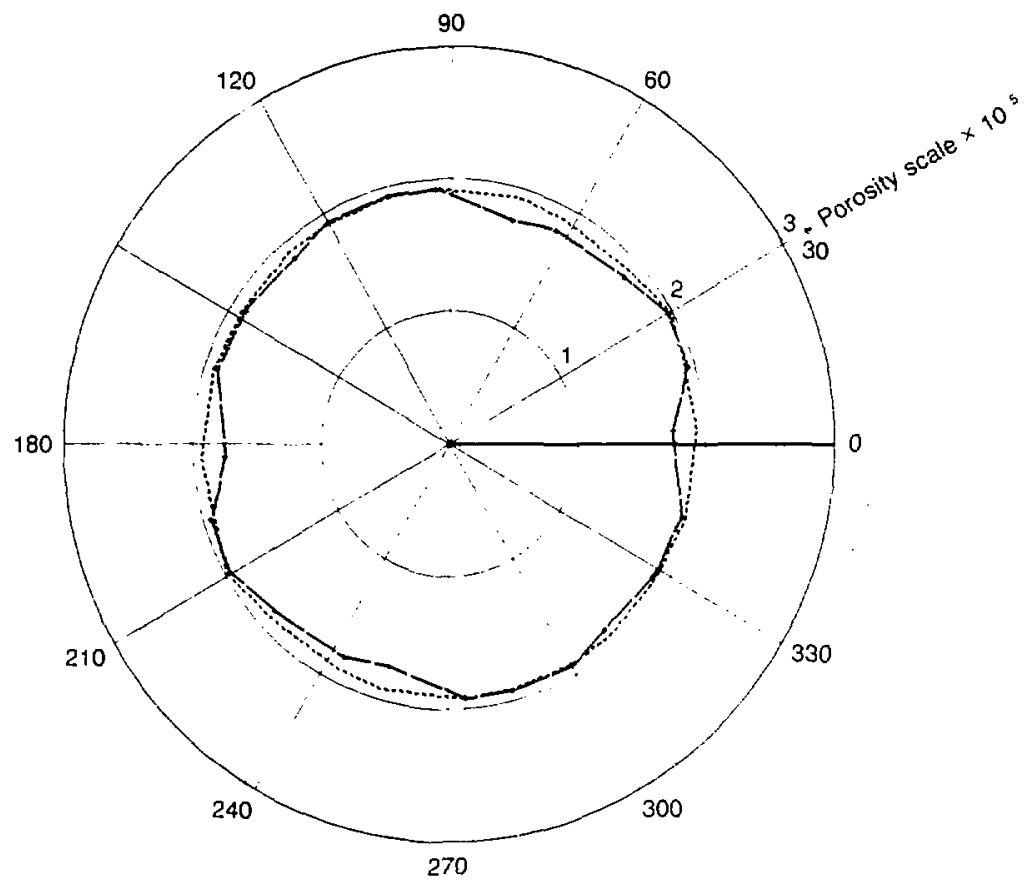

- Hydraulic effective porosity

-...-- Total porosity

$X 2 L 83123424$

Figure 7-29 Polar Plots of Total Porosity and Hydrauljc Effective Perosity for Continuous System With $\sigma_{\mathrm{b}} / \mu_{\mathrm{b}}$ of 0.3 . 


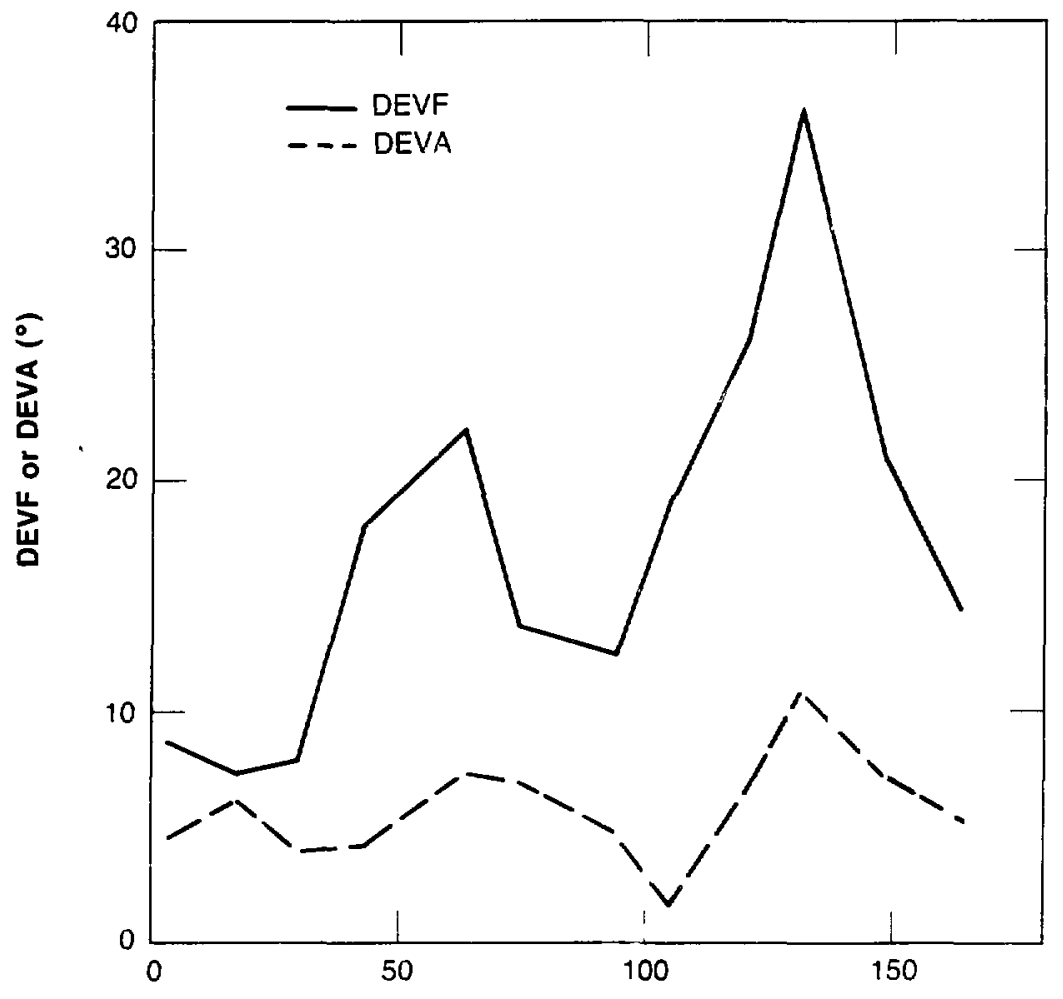

Direction of flow $\left({ }^{\circ}\right)$

XBL $8401-6789$

Figure 7-30 Mean DEVF and Mean DEVA Versus Direction of Flow for Continuous System With $\sigma_{b} / \mu_{b}$ of $C .3$ 


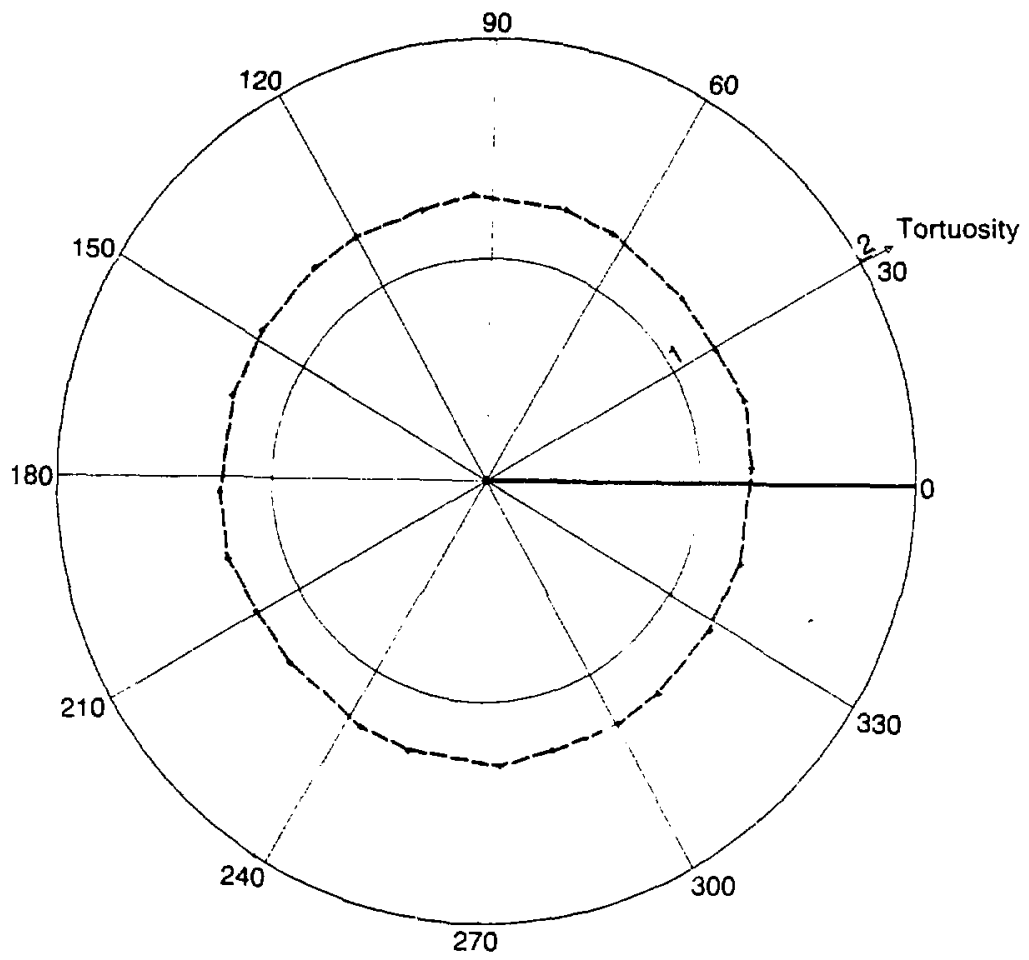

$X 818401-6797$

Figure 7-31 Polar Plot of Tortuosity for Continuous System With $\sigma_{b} / \mu_{b}$ of 0.3 . 
Figure 7-32 shows that polar plot of longitudinal geometric dispersivity. The maximum $\alpha_{\mathrm{L}}$ occurred at $105^{\circ}$. This direction was near the direction of maximum $\alpha_{\mathrm{L}}$ for the continuous system in the constant aperture study. However, the maximum $\alpha_{\mathrm{L}}$ was three times larger for this system than for the system with $\sigma_{\mathrm{b}} / \mu_{\mathrm{b}}$ of 0 . For the continuous system with $\sigma_{\mathrm{b}} / \mu_{\mathrm{b}}$ of 1 , the Peclet number approached infinity, such that $\alpha_{L}$ could not be computed. Thus, the three length-density studies showed that $\alpha_{\mathrm{L}}$ increased as $\sigma_{\mathrm{b}} / \mu_{\mathrm{b}}$ increased.

\subsubsection{Discontinuous System with Mean Fracture Length of $50 \mathrm{~m}$}

The polar plots of total porosity, hydraulic effective porosity, and rock effective porosity are shown in Figure 7-33. All three porosities are directionally stable, and this fracture system behaves like an equivalent porous medium for transport. The mean directional hydraulic effective porosity of 0.0000146 is larger than the rock effective porosity. The three length-density studies showed that hydraulic effective porosity deviated more from rock effective porosity as $\sigma_{b} / \mu_{b}$ increased. The ratios of hydraulic effective porosity to rock effective porosity wPre 1.10 , 1.18 for the systems with $\sigma_{b} / \mu_{b}$ of 0 and 0.3 , respectively. The ratio of $\phi_{H}$ to $\phi_{R}$ was much larger than 2 in most directions for the system with correlated apertures and $\sigma_{b} / \mu_{b}$ of 1 .

Hydraulic effective porosity was greater than rock effective porosity for this discontinuous system, and $\phi_{\mathbf{H}}$ was nearly equal to $\phi_{\mathbf{R}}$ for the previous continuous system. In a discontinuous system, the flow rate in a series of elements is governed by the element with the smallest aperture, such that the large flow capacity of large aperture elements are often negated by small aperture elements. In a continuous fracture system, the flow rate in a fracture is theoretically independent of the llow rates in the fractures intersecting it. Consequently. two different effects of large aperture fracture on mean travel time (flow rate) occur in continuous and discontinuous fracture systems. Continuous fracture systems have, on a relative basis, smaller mean travel times than discontınuous systems because the flow rates in large aperture fractures are not controlled by small aperture fractures. One should keep in mind that travel time in an element is inversely proporional to the flow rate in the element. Since hydraulic effective porosity is equal to q $\overrightarrow{\mathrm{t}} / \mathrm{L}$. hydrauiic effective porosity deviated less from rock effectuve porosity for 


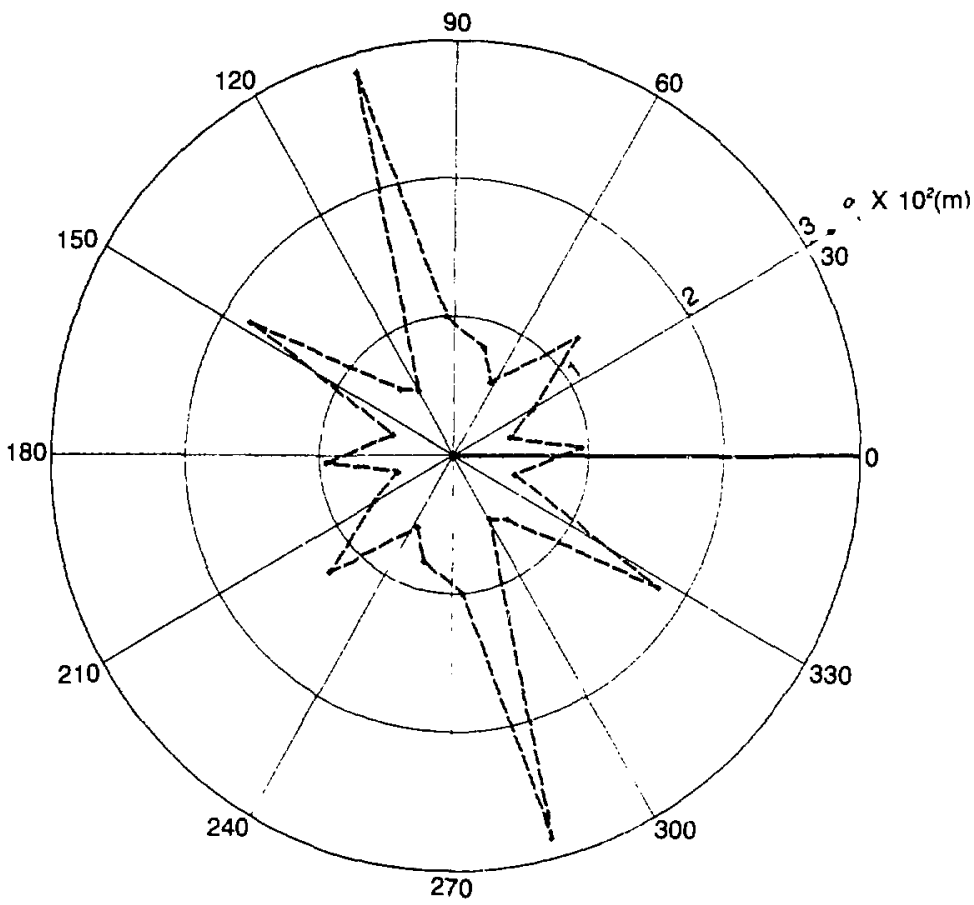

$X B L \quad 84 h-229$

Figure 7-32 Polar Plot of Longitudinal Geometric Dispersivity for Continuous System With $\sigma_{b} / \mu_{b}$ of 0.3 . 


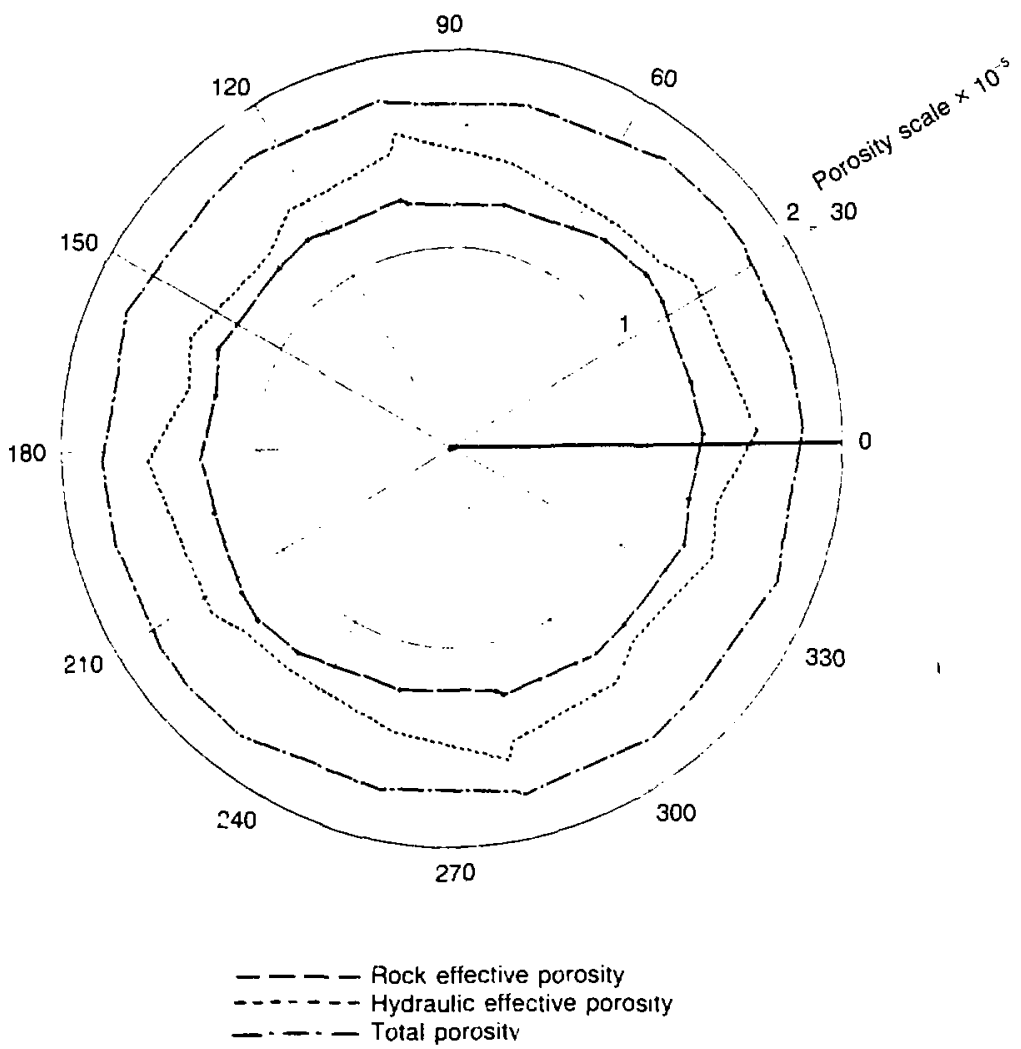

X8L 83127421

Figure 7-33 Polar Plots of Tore! Furosity, Rock Effective Porosity, and Hydraulic Effective Porosity for Discontinuous System With $\mu_{l}$ of $50 \mathrm{~m}$ and $\sigma_{b} / \mu_{b}$ of 0.3 . 
the continuous fracture system.

Figure 7-34 is a plot of mean DEVF and mean DEVA versus direction of flow. DEVF was two times smaller for this system than for the discontinur fstem with $\sigma_{b} / \mu_{b}$ of 1 , and about two times larger than DEVF for the discontinuous system with $\mu_{1}$ of $50 \mathrm{~m}$ in the constant aperture study. Thus, equivalent porous medium flow behavior is more likely to occur for fracture systems with small $\sigma_{\mathrm{b}} / \mu_{\mathrm{b}}$.

The polar plot of longitudina! geometric dispersivity in Figure $7-35$ shows that $\alpha_{\mathbf{L}}$ is relatively constant in most directions. The mean directional $\alpha_{\mathbf{L}}$ is $90.6 \mathrm{~m}$. The mean directional $\alpha_{\mathrm{L}}$ was $65 \mathrm{~m}$ for the discontinuous system with $\mu_{\mathrm{l}}$ of $50 \mathrm{~m}$ in the constant aperture study. The results showed again that $\alpha_{L}$ increased as $0_{b} / \mu_{b}$ increased. Tortuosity was directionally stable, with a mean of 1.365 (Figure 7-36).

\subsection{SUMMARY OF LENGTH-DENSITY ANALYSIS}

The fracture information needed to run the computer program was determined from hydrogeologic data collected at the research site. The geometric parameters of mean fracture length, standard deviation of fracture length, and set arial density could nut be determined from the field data. Consequently, a length-density analysis was conducted to investigate the influence of length and density on mechanical transpor. Since the aperure distribution of a fracture system has a major influence on mechanical transport, three length-density studies were conducted using different apenure distributions: 1) $\sigma_{b} / \mu_{b}$ equal to 0 (S1), 2) $\sigma_{b} / \mu_{b}$ equal to $0.3(\mathrm{~S} 2)$, and 3) $\sigma_{\mathrm{b}} / \mu_{\mathrm{s}}$ equal is, 1 (S3).

The fractuse systems in $\mathrm{S} 1$ and $\mathrm{S} 2$ behaved like equivalent porous media for transport. Hydraulic effe. .ve porosity was nearly equal to rock effective purosity for the continuous sysiems in S1 and S2. For the discontinuous systems in S1 : r:d S2. hydraulic effective porosicy was larger than rock effective porosity, and the Jeviation between hydraulic effective porosity and rock effective porosity increased as the ratio of $\sigma_{\mathrm{b}} / \mu_{\mathrm{b}}$ increased. Hydraslic effect.ive porosity did not equal rock effective porosity in the riscontinuous syst-sns. because large aperture fractures have a different influerce on mechanical transport in discontinuous and continuous 


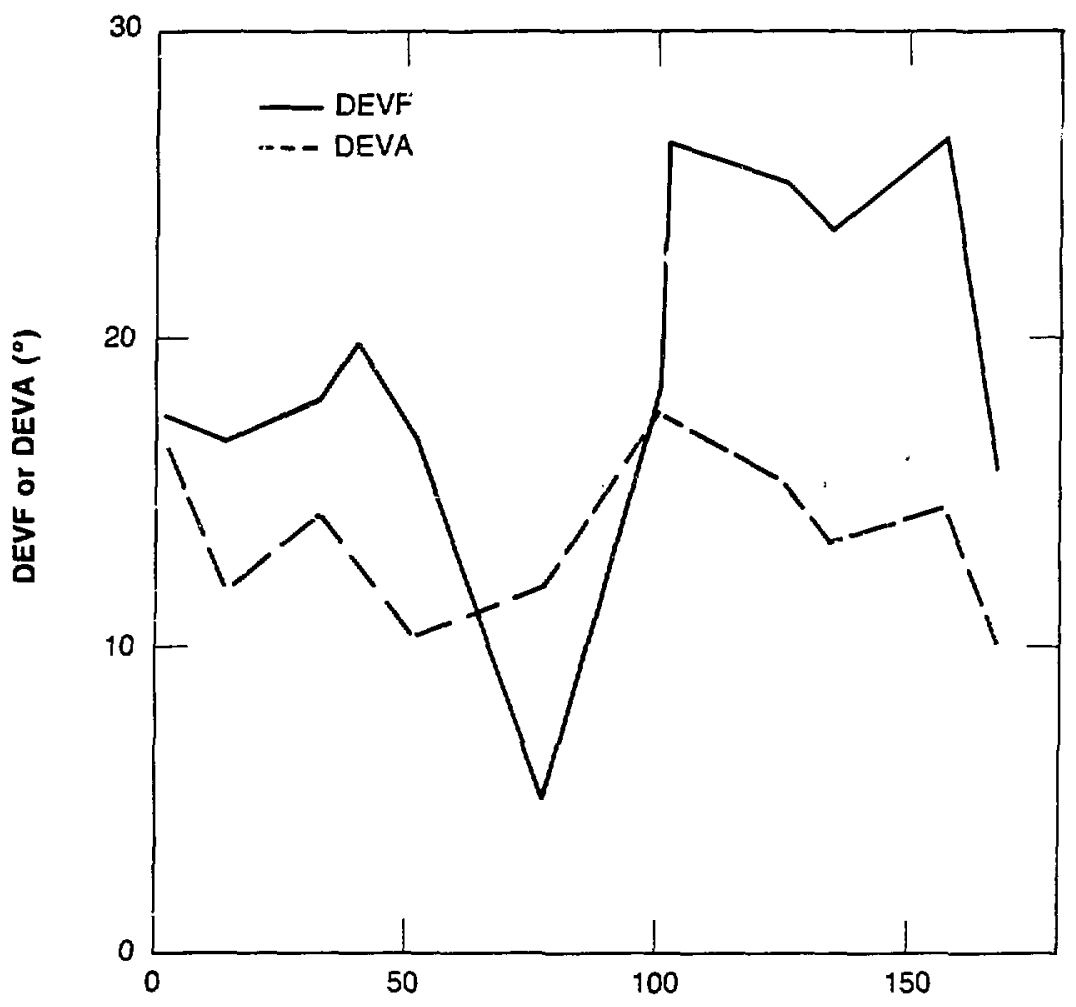

Direction of flow $\left({ }^{\circ}\right)$

XBL 8401-6791

Figure 7-34 Mean DEVF and Mean DEVA Versus Direction of Fow for Discontinuous System With $\mu_{1}$ of $50 \mathrm{~m}$ and $\omega_{b} / \mu_{b}$ of 0.3 . 


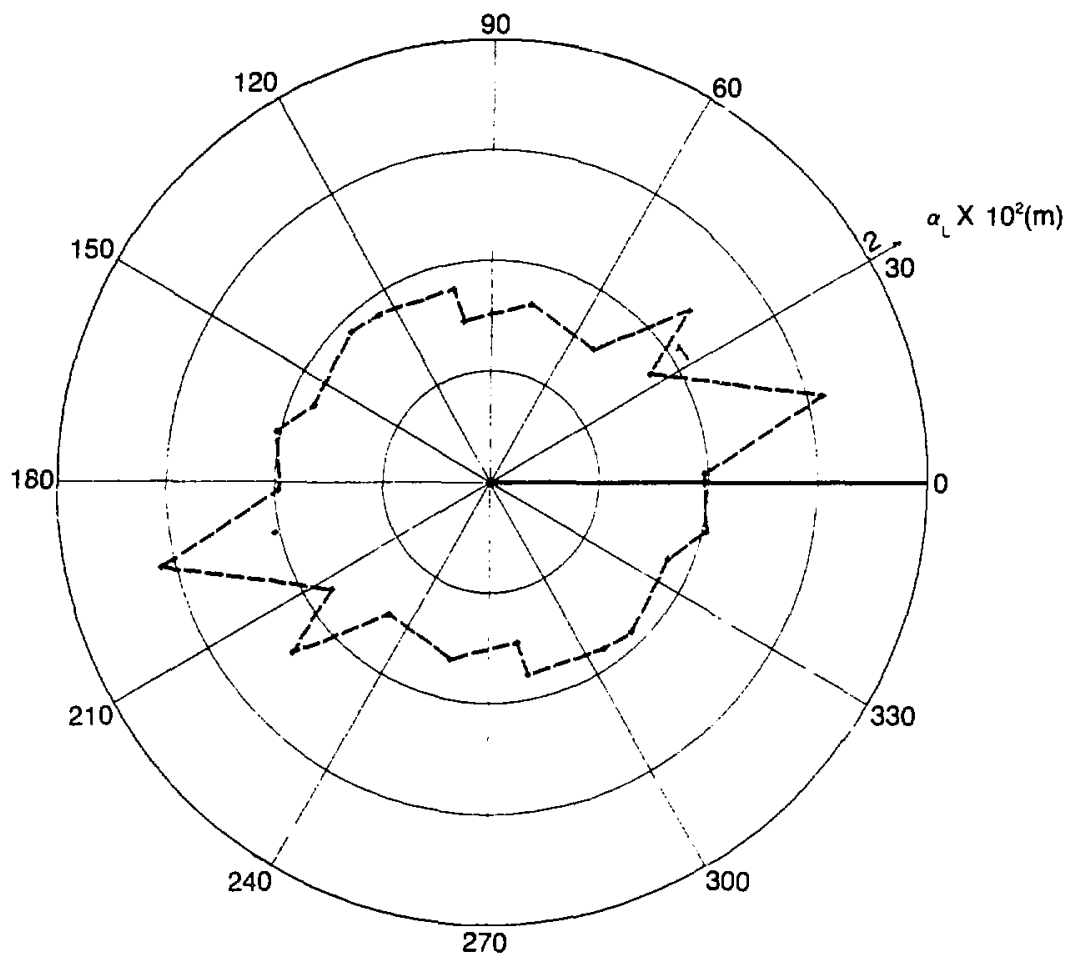

XBL 8401-6798

Figure 7-35 Polar Plot of Longitudinal Geometric Dispersivity for Discontinuous System with $\mu_{1}$ of $50 \mathrm{~m}$ and $\sigma_{\mathrm{b}} / \mu_{\mathrm{b}}$ of 0.3 . 


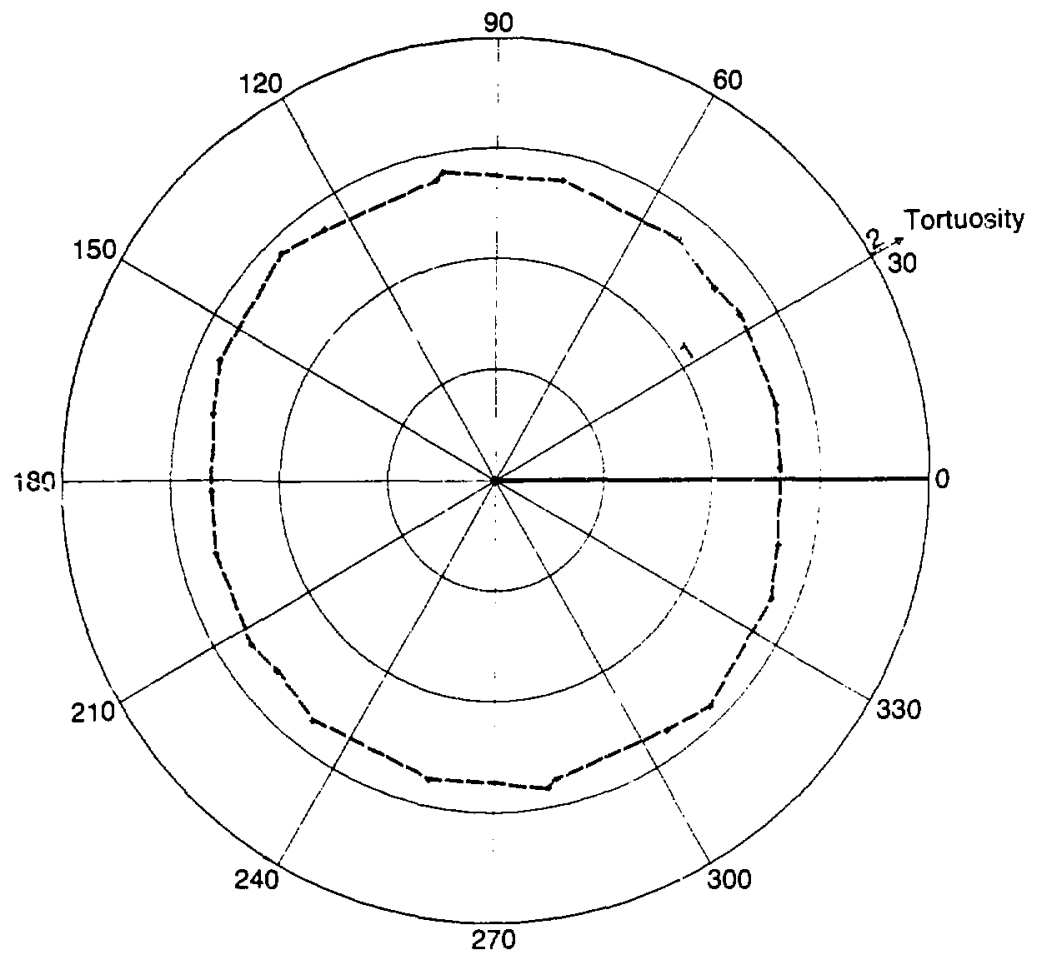

$X B L 8401-6796$

Figure 7-36 Polar Plot of Tortuosity for Discontinuous System With $\mu_{l}$ of $50 \mathrm{~m}$ and $\sigma_{\mathrm{b}} / \mu_{\mathrm{b}}$ of 0.3 . 
systems.

The fracture systems in $\mathbf{S 3}$ did not behave like equivalent porous media for transport. The polar plots of hydraulic effective porosity were jagged because $\phi_{H}$ was directionally dependent. The direction of maximum $\phi_{\mathrm{H}}$ was near $120^{\circ}$ for each system in S3. Hydraulic effective porosity was greater than rock effective porssity for both the continuous and discontinuous systems in S3. The deviations between $\phi_{\mathrm{H}}$ and $\phi_{4}$ were much larger for the fracture systems in S3 than for the discontinuous systems in S1 and S2. Also in S3, the deviations between $\phi_{H}$ and $\phi_{R}$ were larger for the discontinuous system than for the continuous system.

DEVF and DEVA are measures of equivalent porous medium flow behavior in a direction of flow. DEVA and DEVF both increased as $\sigma_{\mathrm{b}} / \mu_{\mathrm{b}}$ increased. Consequently, a fracture system with a narrow aperture distribution (small $\sigma_{\mathrm{b}}^{\prime} \mu_{\mathrm{b}}$ ) behaved more like an equivalent porous medium than a fracture system with a wide aperture distribution

Tortuosity was directionally stable in all studies. A directionally stable tortuosity indicated that the fracture systems were fairly isotropic. Tortuosity was slightly less in the continuous systems than in the discontinuous systems. Fluid flows in a more direct path across the flow region in a continuous system that in a discontinuous system because the fractures in a continuous system span across the entire flow region. Consequently, tortuosity was smaller for the continuous systems.

The longitudinal geometric dispersivity is theoretically constant in all directions for an equivaren: isotropic porous medium. However, the longitudinal geometric dispersivities were directior lly dependent for the fracture systems in S1 and S2, and no general directional relationship for $\alpha_{\mathbf{L}}$ was found. Each fracture system had a unique polar fivt for $\alpha_{L}$. However, we found that mean directional $\alpha_{\mathrm{L}}$ was independent of mean fracture length for the discontinuous system in SI, even though the polar plots of $\alpha_{\mathrm{L}}$ were different. These systems were not the only nearly isotropic systems which showed directionally dependent longitudinal geometric dispersivities in this research. The equivalent isotropic continuous system of two orthogonal sets of fractures in section 5.3 also had a directionally varying $\alpha_{L}$. Thi aperture distribution greatly 
influenced longitudinal geometric dispersivity as $\alpha_{\mathrm{L}}$ could not be computed for the fracture systems in $\mathbf{S} 3$ because the Peclet number approached infinity. The three length-density studies showed that $\alpha_{\mathrm{l}}$ increased as $\sigma_{\mathrm{b}} / \mu_{\mathrm{b}}$ increased. 


\section{CHAPTFR 8 \\ CONCLUSIONS AND RECOMMENDATIONS}

\subsection{CONCLUSIONS}

The primary objectives of this research were to investigate directional mechanical transport parameters fer anisotropic fracture systems, and to determine when a fracture system can be treated like an equivalent porous medium for mechanical transport. The thi essential conditions necessary for measuring directional mechanical transport for an equivalent porous medium are a uniform flow field, and a test section where linear length is constant. When these two conditions are satisfied, mechanical transport parameters can be measured from the breakthrough curve for the fluid that flows within the test section.

Parameters were defined to evaluate directional mechanical transport. The mean rate of advection in a particular direction was chararterized by the bydraulic effective porosity. The hydraulic effective porosity was defined as the ratio of specific discharge to average linear velocity. In porous media transport studies, hydraulic effective porosity is assumed to be direction$\downarrow$ a'ly stable. Thus, the shape of the polar plot of hydraulic effective porosity was used to test whether a fracture system behaved like an equivalent porous medium for mechanical transport.

The mechanical dispersive flux in a particular direction was characterized by the longitudinal geometric dispersivity. The longitudinal geometric dispersivity is a function of several components of the georetric dispersivity tercor. The deriviton of tha reiationst.; betwent longitudinal geometric dispersivity and this tensor showed that it is nci necessary to determine all the components of this fourth-ranked tensor to evaluate longitudinal mechanical transport. In porous media transport studies, an anisotropic medium is treated as an equivalent isotropic medium. When this simplifiration is made, the longitudinal geometric dispersivity becomes directionally stable. We have found that the directional variation of the longitudinal geometric dispersivity can be significant for anisotropic fracture systems. Furthermore, a fracture syster. which behaved like an equivalent isotropic porous medium for fluid flow did not have a 
directionally-stable longitudinal geometric dispersivity.

Another important mechanical transport parameter we studied was the tortuosity. Tortuosity was first used to predict permeability. Tortuosity can also be used to predict the effective diffusion coefficient and to estimate the mean pore velocity. Little work has been carried out on the directional characteristics of tortuosity.

The fact that fluid flow and mechanical transport are coupled made it necessary to investigate equivalent porous medium behavior for fluid flow. When a fracture system, under a constant hydraulic gradient, behaves like an equivalent porous medium, the specific aischarge will be uniform in any direction of flow. The uniformity of the specific discharge in a direction was evaluated using the continuity test and the angle of flow test. Darcy's law also specifies that the flow field in any direction of flow can be predicted by an unique permeability tensor. If such a tensor exists, the square root of permeability in the direction of now plots as an ellipse. Thus, the shape of the square root of permeability was used to test whether directional flow for the system behaved like an equivalent forous medium.

A numerical model was developed to simulate mechanical transport under steady laminar flow in a network of fractures. The model incorporated the principles of laminar flow to calcllate the paths of streamtubes for fluid traveling from one side of a flow region to another. We assumed that fluid how was restricted to planar fractures within an impermeable rock matrix. and that mechanical transport was the only transport process. Of course, other transport processes occur, but focusing on mechanical transport led to the evaluation of the directional transport properties for anisotropic systems.

Mechanical transport was first invesilgated for regular, anisotropic systems of continuous iractures. These fracture systems were first studied because they behaved like equivalent porvus media for fluid flow, with flow parameters that could be analytically computed. The results showed that a fracture system which bekaved as a continuum for fluid flow did not behave as a continuum for mechanical transpor. This was demonstrated by a hydraulic effective porosity which was directionally dependent and that decreased well below the porosity in 
certain directions. In these directions, part of the fracture voids became nonconductive because the direction of the hydraulic gradient was perpendicular to the orientation of a fracture set.

The size of the mechanical transport continuum was found to be directly related to tortuosity. As tortuosity increased, travel paths became more irregular and deviated more from the mean direction of fow. Consequently, larger flow regions were needed befinre representative mechanical transport behavior occurred when tortuosity was large.

Tortuosity did not behave as one would expect for an equivalent porous medium for these continuous fracture systems. Tortuosity decreased to a minimum of 1.0 in a direction where one fracture set became nonconductive. This direction was not necessarily in the direction of maximum permeability, where one would expect tortuosity to be a minimum for an equivalent porous rnedium. Thus, porous medium equivalence can be found for certain parameters, while the directional properties of other parameters show that porous medium equivalence is not possible.

The longitudinal geometric dispersivity showed large and abrupt directional variations for the continuous systems. The magnitude of the maximum langitudinal geometric dispersivity increased with the degree of anisotropy, and no general relationship was found between the principal directions of longitudinal ciispersivity and the principal directions of permeability. Thus, both longitudinal geometric dispersivity and hydraulic effective porosity were highly directionally dependent. The use of a directionally stable longitudinal geometnc dispersivity cannot be justified for these systems. Serious errors in transport prediction would result if one were to tre th these anisotropic systems as equivalent isotropic systems.

The first two discontinuous fracture systems studicd (section 6.2 and 6.3 ) behaved like equivalent porous media for fluid flow. Hydraulic etfective porosity for bcth systems showed a slight directional dependeries, with the minimum hydraulic effective porosity occurring near the direction of maximum permeability. The polar plots of hydraulic effective porosity, however. were nearly circular and we concluded that each fracture system behaved like an equivalent porous medium for transport. The mean directional hydraulic effective porosity for each sys- 
tem was greater than the rock effective porosity so the average linear velocity was less than the usual porous medium estimate of specific discharge divided by rock effective porosity. Hydraulic effective porosity was larger than the rock effective porosity because zones of slow movement existed in the flow region which caused mean travel time to be large in relation to linear travel length and specific discharge. For these two discontinuous systems, tortiosity increased from the direction of maximum permeability to the direction of minimum permeability, as one would expect for equivalent porous media.

Tortuosities as high as 3.8 were measured for the discontinuous system of two sets oriented at $0^{\circ}$ and $30^{\circ}$. In the literature, tortuosity is reported to normaily range between 1 and 2. The large measured values of tortuosities were caused by the high anisotropy of the system, as the ratio of maximum permeability to minimum permeability was ten. The size of the fluid flow continuum was found to be related to tortuosity. As tortuosity increased, hoth DEVA and DEVF, measures of equivalent porous medium flow behavior, increased. Thus, the continuum size for both fluid flow and mechanical transport was found to be inversely related to tortuosity. VPORE computed as the product of tortuosity and average linear velocity was shown to be a good estimate of the mean pore velocity. The actual mean pore velocity is normally extremely difficult to determine so an estimate like VPORE is used.

Each discontinuous system showed a unique directional dependence for the longitudinal geometric dispersivity. The ratio of maximum to minimum longitudinal geometric dispersivity was directly related to the degree of anisotinfy. The directional variations in longitudinal geometric dispersivity for the discontinuous systems were not as severe as those in the continuous fracture systems. However, the two discontinuous fracture systems showed again that the determination of the directional transport properties for an anisotropic medium is essential to predicting uransport.

The large differences in longitudinal geometric dispersivity measured from laboratory and field data could be due to the directional nature of $\alpha_{\mathrm{L}}$. Longitudinal geometric dispersivity is properly measured in the laboratory when the procedure described in sections 3.5 and 3.6 is 
uscd. We are unaware of any laboratory experiments which has usea this procedure. In a groundwater aquifer, the natural direction of flow may vary spatially such that, to model transport in the aquifer, a directional $\alpha_{\mathrm{L}}$ must be used. In most transport studies, a constant $\alpha_{\mathrm{L}}$ is used to model transport in the aquifer. Consequently, the dispersivities measured from laboratory and field data may not properly characterize transport in the aquifer.

The connectivity sensitivity studies for mean fracture length, set areal density, and mean fracture orientation showed that mechanical transport parameters were generally much more sensitive to the three connectivity parameters than either specific discharge or rock effective porosity. For example, both mean travel time and the variance of the breakthrough curve were highly sensitive to each connectivity parameter, and no relationship was found between either mechanical transport paraneter and any connectivity parameter. However, specific discharge and rock effective porosity both increased with connectivit:. Tortuosity was the only mechanical transport parameter that showed no sensitivity to connectivity. The least sensitive connectivity parameter was the mean orientation of the fracture set, with the mean fracture length and the set areal density having about equal sensitivity. In the mean aperture sensitivity study, the variance of the breakthrough curve was the most sensitive mechanical transport para: ieter, followed by mean travel time. The longitudinal geometric dispersivity and tortuosity were both insensitive to mean aperture.

Connectivity increased when fracture orientations became distributed, while connectivity decreased when fracture lengths became distributed. The increased connectivity created a more random fracture system, and the following parameters increased because of this: rock effective porosity, specific discharge, tortuosity. mean travel time, and the variance of the breakthrough curve. Heterogeneity increased when fracture apertures became distributed. This increased heierogeneity caused a reduction in the specific discharge. The specific discharge decreased because the flow rate in a series of fractures is governed by the fracture with the smallest aperture. The smaller flow rate meant that fluid particles took a longer time to travel through the flow region, and thus, mean travel time increased. it he variance in the breakthrough curve 
increased because the increased heterogeneity caused a greater variation in pore velocities.

A length-density analysis was conducted using fracture data collected at a research site in Manitoba, Canada. Since the aperture distribution of a fracture system has a major influence on mechanical transport, three length-density studies were conducted using different aperture distributions. The studies showed that porous media equivalence for fluid flow and transport is likely to occur for fracture systems in which the ratio of $\sigma_{\mathrm{b}}$ to $\mu_{\mathrm{s}}$ is small. For the continuous systems which showed porous media equivalence, hydraulic effective porosity was nearly equal to rock effective porosity. However, hydraulic effective porosity was larger than rock ef "ctive porosity for the discontinuous systems which exhibited porous media equivalence. The larger deviation between hydraulic effective porosity and rock effective porosity in the discontinuous systems was caused by the different effects of large aperture fractures on travel time in continuous and in discontinuous systems. Tortuosity was directionally stable for all systems which indicated that each fraciure system was fairly isotropic.

Theoretically, the Iongitudinal geometric dispersivity is directionally stable for an equivalent isotropic medium. However, the longitudinal geometric dispergivities were direcionally dependent for the fracture sysiems which otherwise exhibited porous media equivalence. We found that the mean directional $\alpha_{I}$ was independent of mean fracture length for the discontinuous systems in the constant aperture length-density studv, even though the polar plots of $\alpha_{\mathrm{L}}$ were different. These systems were not the only nearly isotropic systems which showed directionally varying longitudinal geometric dispersivities in this research. The equivalent isotropic system of two orthogonal sets of continuous fractures in section 5.3 also had a directionally dependent $\alpha_{L}$. The aperture distribution greatly influenced longitudinal geometric dispersivity, as $\alpha_{L}$ could not be computed for the fracture systems in which the mean aperture cqualed the standard deviation of aperture. The Peclet number approached infinity for these fracture systems. The three length-density studies showed that $\alpha_{\mathrm{L}}$ increased as $\sigma_{\mathrm{b}} / \mu_{\mathrm{b}}$ increased. 


\subsection{RECOMMENDATIONS}

The purpose of this research was to investigate the directional measurement of mechanical transport for anisotropic fracture systems. No extensive study of directional mechanical transport in groundwater systems has been repurted in the literature. Consequently, in this research a set of experiments was developed to meisure directional mechanical transport, and then it was shown how directional mechanical transport can be interpreted from these experiments. The primary mechanical transport parameters investigated were hydraulic effective porosity, tortuosity, and longitudinal geometric dispersivity.

This study has uncovered a number of significant findings on directional transport properties. For example, tortuosities much larger than three were measured in this study. In most transport studies, tortuosity is rarely determined, and is normally assigned a value about 1.4 . Further research is needed to understand how travel paths influence fluid flow and transport. Tortuosity was used in this work to characterize the mean path length. Research is needed to determine how the distribution of paths lengths can be used in evaluating transport. Another area that can be investigated is the use of the Kozeny equation for anisotropic media. The computer program can be modified so that the shape factor and the hydraulic radius in the Kozeny equation can also be computed for different directions of flow.

The longitudinal geometric dispersivity was shown to be highly directionally dependent. Yet, in most transpurt studies, a constant longitudinal geometric dispersivity is assumed in all directions, as the anisotropic medium is treated as an equivalent isotropic medium. Future research can investigate the error that is made when this assumption is used. The error associated with this assumption can be determined by conducting tracer experiments for aniso:ropic media using the hydraulic boundary conditions for isotropic media described in section 2.2.2.

Directional mechanical transport is measured in this study using a numerical model. An alternative method would be to conduct laboratory experiments. The conventional laboratory tracer exf'riment consists of applying no-flow boundary conditions along the sides of the test sample, and constant-head boundary conditions across the ir.let and the outlet to the sample. 
Tracer is next injected across the inlet, and then the breakthrough curve is constructed to measure transport. The conventional tracer experiment can be used to measure mechanical transport in the two principal directions. Between the two principal directions, the tracsr experiments describeu in Chapter 3 must be used to measure mechanical transport. The crucial step in designirit this laboratory experiment is the application of the constant hydaulic gradien boundary conditions along the sides of the test sample. An innovative design is needed to implement such hydraulic boundary conditions. Once the hydraulic boundary conditions have been applied, the experiment can proceed in the manner described in section 3.6.

The computer program in Chapter 4 was written using single precision. Certain parts of the computer program require computational accuracy. For example, the flow rate in each streantube must be accurately computed to ensure that the total flow rate into a node is equr:l to the total tlow rate out of a node. Be:ter accuracy can be gained by triting the computer program using double precision. However, the dimensions of variable arrays are reduced when double precision is used and his will result in an analysis of smaller sized problems unless a computer with a larger storage capacity becomes available. To increase accuracy without decreasing the size of the arrays used in the present computer program, one should switch from the VAX machine (Ciass IV) to a Class VI machine such as the Cray.

The accuracy of the modeling results also depends on the availability of detailed and reliable fracture data. Wc have shown that the statistical parameters of the aperture distribution are essential for modeling fluid flow and mechanical transport. To obtain a representative sample of the aperiure distribution, a well planned set of packer tests would have to be conducted in the fractured rock mass. Thus, a dynamic interaction between theortical (modeling) and experimental (field data) groundwater research activities is essential to significant advancements in this field.

A useful addition to the computer program would be to eliminate all dead-end locps in the fracture netrork formed by nonconauctive fracture segments before the mechanical transport simulation stage. The program would not have to search for dead-end loops in the pro- 
cess of locating streamtubes, and thus, computer time would decrease for this stage. The algorithm for eliminating dead-end loops should follow the fracture system generation stage. Tne elimination of dead-end lcops would then decrease the number of elements and nodes in the flow region, ard the bandwidth in the conductance matrix would probably decrease. Thus, computer time would also decrease $\mathrm{ir}$ the hydraulic head calculation stage.

The next step in the theoretical development would be to determine if directional hydrodynamic dispersion can te analyzed. The investigation of directional hydrodynamic dispersion would require the understanding of another transport process, molecular diffusion. The two key steps that must be sonsidered in developing the theory to measure Airectional hydrodynamic dispersion are: 1) findirg the apprci-iate test section in which to reasure hydrodynamic dispersion, and 2) determining how syúrodynamic dispersion parameters can be evaluated from measurements made within this test section. The concepts of directional mechanical transpor: in Chapter 3 will provide a good starting point for developing the necessary theory.

The present computer program would have to be modified to model hydrodynamic dispeision. Numerical methods that are commonly used to model hydrodynamic dispersion in po. ol: media are the finite difference method (FDM), the finite element method (FEM), and the method of characteristics (MOC). If the present computer program were to be modified to include hydrodynamic dispersion, the MOC would be the best me: . be able to use some of the streamtubing principles ueveloped in Chapter 4. Also, thr MOC requires less computer time than either the FEM or the FDM. Both the FDM ard the FEM require the solution of a larger matrix than the conductance matrix for fluid flow. Furthermore, this matrix would have to be solved for each time step since transport is a transient phenomenon.

The computer program as developed in this work for hydrndynamic dispersion should be used in two parts. First. the computer program should be used to evaluate directional tnechanical sransport and evaluar: porous medium equivalence. Mechanical transport must be 
evaluated first because the direction of fiow, average linear velocity, and tortuosity are needed to model hydrodynamic dispersion. Then the computer program should be used to evaluate hydrodynanic dispersion. In this manner, the program can be used to evaluate both mechanical transport and hydrodynamic dispersion. 


\section{REFERENCES}

Anderson, M.P. (1979) Using models to simulate the movement of contaminants through groundwater flow systems. Critical Reviews $i$. Environmental Resour. Res., 17(4), 1075 1081.

Baird, D. C. (1962) Experimentation: an introduction to measurement theory and experiment design, Prentice-Hall, New Jersey, 198.

Biggar, J. W., and D. R. Nielsen (1960) Diffusion effects in miscible displacements occurring in saturated and unsaturated porous materials, J. Geophys. Res., 65(9), 2887-1895.

Carman, P. C. (1956) Flow of gases through porous media, Academic Press, New York, 182.

Castiilo, E., R. J. Krizek, and G. M. Karadi (1972) Comparison of dispersion characteristics in fissured rocks, IAHR Proc. Second Symposium on Fundamentals of Transport Phenomena in Porous Media, 778-797.

Cherry, J. A., R. W. Gilham, and J. F. Pickens (1975) Contaminant hydrogeology, part I physical processes, Gcoscience Canada, 2(2), 76-84.

Childs, K. E., S. B. Upchurch, B. Ellis (1981) Sampling of variable, waste- migration patterns in groundwater, Groundwater, 12(6), 369-377.

Collins, R. E. (1961) Flow of fuids through porous materials, The Petroleum Publishing Company, Tulsa, 270.

Dieulin, A., G. Mathercs, and G. de Marsily (1981) Growth of the dispersion coefficient with the mean traveled distance in porous media, The Science of the Total Environment, 21, 319-328.

Dullien, F. (1979) Porous media, fluid transport and pore structure, Academic Press, New York, 306.

Ellis, W. R., L. Kevi, and W. A. Wiebenga (1968) The investigation of water flow through porous mediums by means of radiouracers, Water Resour. Res., 4(2), 413-416.

Freere, R. A., and J. A. Cherry (1979) Groundwater, Prentice-Hall, New York, 604.

Fried, J. J., (1975) Groundwater Pollution, Elsevier Scientific, Amsterdam, 930.

Gelhar, L. W., A. L. Gutjahr, and R. L. Naff (1979) Stochastic analysis of macrodispersion in a stratified aquifer, Water Resour. Res., 15(6), 1387-1397.

Gillham, R. W., and J. A. Cherry (1982) Contaminant migration in saturated unconsolidated geologic deposits, Recents Trends in Hydrogeology. Geological Society of America Special Paper 189, 31-63.

Gill, W. N., V. Guceri, and R. J. Nunge (1559) Laminar dispersion in diverging channels in concentric annuli. Office of Saline Water, Research and Development Report No. 443.

Grove, D. B., and W. A. Beetem (1971) Porosity and dispersion constant calculations for a fractured carbonate aquifer using the two well tracer method. Water Resour. Res., 7(1), 128-134.

Haring, R. E., and R. A. Greenkorn (1970) A statistical model of a porous medium with nonuniform pores, A.I.C'.E. 16(3), 477-482. 
Hazzaa, I. B., K. F. Saad, R. K. Girgis, A. A. Bakr, and F. M. Swailem (1964) Determin ation of porosity of groundwater aquifers by the radioactive tracer technique, Int. J. Apps. Radiat. Isotopes, 16, 26!-165.

Hazzaa, I. B., R. K. Girgis, K. F. Saad, F. M. Swailem, and A. A. Bakr (1966) Study of the applicability of different radioisotopes for determination of the porosity of ground-wate: aquifers, Int. J. Appl. Radiat. Isotopes, 17, 621-628.

Hoehn, E., and P. V. Roberts (1982) Advection-dispersion interpretation of tracer observations in an aquifer, Groundwater, 20(4), 457-465.

Karadi, G. M., R. J. Krizek, and E. Castillo (1972) Hydrodynamic dispersion in a single rock joint, J. Appl. Phys., 43(12), 5013-5021.

Krizek, R. J., G. M. Karadi, and E. Socias (1972) Mathematical model for two-dimensional percolation through fissured rock, Proc. Int. Soc. Rock Mech. Symp. on Percolation Through Fissured Rock Stuggart, TC1-TC15.

Levenspiel, O. (1972) Chemical reaction engineering, John Wiley, New York, 578.

Long, J. C. S. (1982) Verification and characterization of continuum behavior of fractured rock at AECL underground research laboratory, task 1: investigation of equivalent porous media behavior for networks of discontinuous fractures, report on activities for FY 1982, LBL-14975, Lawrence Berkeley Laboratory.

Long, J. C. S. (1984) Permeability and porosity in three vertical planes near the URL shaft site, Letter Report in preparation.

Long, J. C. S., J. S. Remer, C. R. Wilson, and P. A. Witherspoon (1982) Pornus media equivalents for network of discontinuous fractures, Water Resour. Res., 18(3), 645-658.

Marcus, H., and D. E Evenson (1956) Directional permeability in anisotropic porous media, Water Resour. Center Contrib. No. 31, University of California, Berkeley, 105.

Matheron, G., and G. de Marsily (1980) Is transport in porous media always diffusive? a counter example, Water Resour. Res., 16(5), 801-817.

Neretnieks, I. (1980) Some difficulties in interpreting in-situ tracer tests, Symp. on the Scientific Basis for Nuclear Waste Management, Boston, 10.

Ogata, A. (1970) Theory of dispersion in a granular medium, U. S. Geol. Surv. Prof. Paper $411-1,34$.

Parsons, R. W. (1966) Permeability of idealized fractured rock, Soc. Pet. Eng. Jour., 6(2), 126136.

Perkins, T. K, and O. C. Johnston (1963) A review of diffusion and dispersion in porous media, Soc. Pet. Eng. Jour., 70-84.

Pickens, J. F., and G. E. Grisak (1981a) Modeling scale-dependent dispersion in hydrogeolngic systems, Water Resour. Res., 17(6), 1701-1711.

Pickens, J. F., and G. E. Grisak (1981b) Scale-dependent dispersion in a stratified granular aquifer, Water Resour. Res., 17, 1191-1211.

Rice, D. A., D. J. Fontugne, R. G. Lattini, and A. J. Barduhn (1969) Anisotropir permeability in porous media,Proc. Sixth State-of-the-Art Symp. sponsored by' Industrial and Engineering Chemistry of American Chemistry Society, 48-56. 
Scheidegger, A. E. (1960) The physics of flow through porous media, MacMillan Company, New York, 313.

Scheidegger, A. E. (1961) General theory of dispersion in porous media, Jour. Geophys. Res., $66(10), 3273-3277$.

Scheidegger, A. E. (1964) Statistical hydrodynamics in porous media, Advan. Hydrosci., 1, 161-181.

Schwartz, F. W. (1977) Macroscopic dispersion in porous media: the controlling factors, Water Resour. Res., 13(4), 743-752.

Schwartz, F. W., L. Smith, and A. S. Crowe (1981) Stochastic analysis of groundwater flow and contaminant transport in a fractured rock system, Proc. Material Research Society, Symposium on the Scientific Basis of Nuclear Waste Management, Boston.

Smith, L., and F. W. Schwartz (1980) Mass transport, 1, a stochastic analysis of macroscopic dispersion, Water Resour. Res., 16(2), 303-313.

Smith, L, and F. W. Schwarz (1981a) Mass transport, 2, analysis of uncertainty in prediction, Water Resour. Res., 17(2), 351-369.

Smith, L., and F. W. Schwartz (1981b) Mass transport, 3, role of hydraulic conductivity data in prediction, Water Resour. Res., 17(5), 1463-1479.

Snow, D. T. (1969) Anisotropic permeability of fractured media, Water Resour. Res., 17(3), 555-564.

Sudicky, E. A., and J. A. Cherry (1979) Field observations of tracer dispersion under natural flow conditions in an unconfined sandy aquifer, Water Pollut. Res. Can., 14, 1-17.

Taylor, G. I. (1953) Dispersion of soluble matter in solvent flowing slowly through a tube, Proc. R. Soc. London Ser. A, 219, 186-203.

Topping, J. (1955) Errors of observation and their treatment, Reinhold Pulbishing Corp.. New York, 119.

Van der Laan, E. (1958) Notes on the diffusion-type model for the longitudinal mixing in flow, Chem. Eng. Sci., 7, 187-191.

Von Rosenburg, D. U. (1956) Mechanics of steady state single-phase fluid displacement from porous media, A.I.Ch.E. Journal, ż(1), 55-58.

Walters, G. R., H. W. Bentley, and J. J. Ward (1983) Continuum and non-continuum aspects of groundwater movement at the waste isolation pilot plant, Southeastern New Mexico, EOS Trans. Amer. Geophys. C'nion, 64(45), 713.

Warren, J. E. and F. F. Skiba (1964) Macroscopic dispersion, Soc. Pet. Eng. Jour., 215-230.

Wilson, C. R. (1970) An investigation of laminar fow in fractured porous rocks. Pn.D. thesis, University of Ca!'fornia, Berkeley, 178

Wyllie, M. R. J., and M. B. Spangler (1952) Application of electrical resistivity measurements to problem of fluid flow in porous media. Bulleth of the American Association of Petroleum Geologists, 36(2), 359-403. 
This report was done with support from the Depariment of Energy. Any conclusions or opinions expressed in this report represent solely those of the aul hor(s) and not necessarily those of The Regents of the University of California. the Lawrence Berkeiey Laboratory or the Department of Energy.

Reference to a company or product name does not imply approval or recammendation of the product by the University of California or the U.S. Department of Energy to the exclusion of others that may be suitable. 